
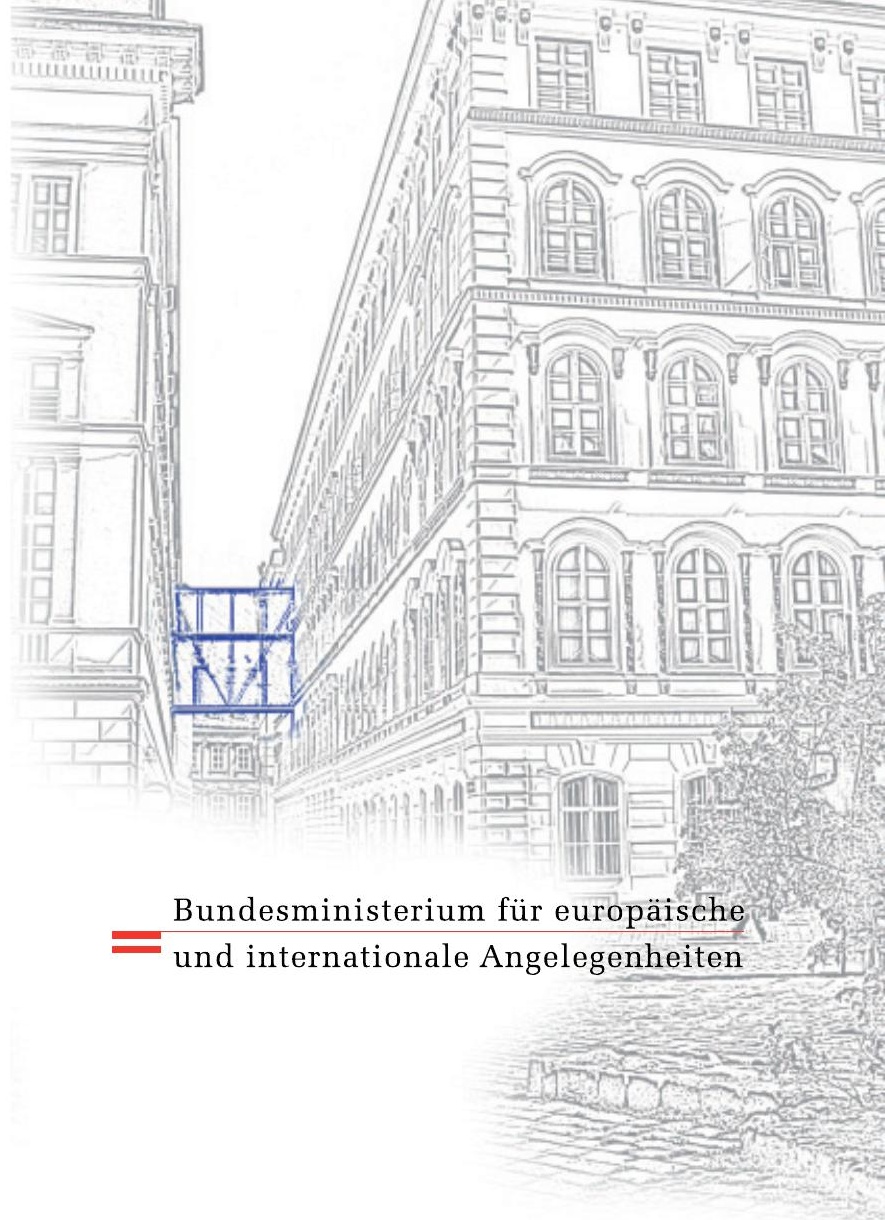

\title{
Österreich im Sicherheitsrat der Vereinten Nationen
}

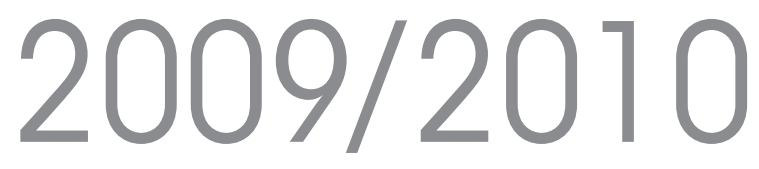

Bericht des Bundesministers für europäische und internationale Angelegenheiten 


\begin{tabular}{|c|c|}
\hline & $\begin{array}{l}\text { Bundesministerium für europäische } \\
\text { und internationale Angelegenheiten } \\
\text { Minoritenplatz } 8 \\
\text { A-1014 Wien }\end{array}$ \\
\hline Telefon: & $\begin{array}{l}\text { an Werktagen in der Zeit von } \\
9 \text { bis } 17 \text { Uhr: } \\
\mathbf{0} \mathbf{5 0} \mathbf{1 1} \mathbf{5 0 - 0} \text { / int.: } \mathbf{+ 4 3} \mathbf{5 0} \mathbf{1 1} \mathbf{5 0 - 0} \\
\text { alternativ: } \\
\text { (01) } \mathbf{9 0} \mathbf{1 1 5 - 0} \text { / int.: (+43-1) } \mathbf{9 0} \mathbf{1 1 5 - 0} \\
\text { kostenfreies Anrufservice: } \\
\text { (0800) } 234 \mathbf{8 8 8} \text { (aus dem Ausland nicht wählbar) }\end{array}$ \\
\hline Fax: & $\begin{array}{l}\text { 0 } 501159-0 \text { / int.: +43 } 501159-0 \\
\text { alternativ: } \\
\text { (01) } 90420 \text { 16-0 / int.: (+43-1) 904 20-16-0 }\end{array}$ \\
\hline E-Mail: & post@bmeia.gv.at \\
\hline Internet: & www.bmeia.gv.at \\
\hline
\end{tabular}

\section{Weiterführende Links zu den Vereinten Nationen:}

Weitere Informationen zu den Aktivitäten der Vereinten Nationen im Internet:

- Vereinte Nationen: www.un.org

- Sicherheitsrat der Vereinten Nationen: www.un.org/Docs/sc

- Ständige Vertretung Österreichs bei den Vereinten Nationen in New York: www.aussenministerium.at/newyorkov 


\section{Österreich im Sicherheitsrat}

\section{der Vereinten Nationen}

\section{9/2010}

Bericht des Bundesministers für europäische und internationale Angelegenheiten 
Medieninhaber und Herausgeber:

Bundesministerium für europäische und internationale Angelegenheiten

Minoritenplatz 8, 1014 Wien

Für den Inhalt verantwortlich:

Ges. Dr. Peter Huber

Leiter der Abteilung für Internationale Organisationen

Technische Koordination:

Ges. Mag. Christoph Weidinger

Abteilung für Presse und Information

Gesamtherstellung:

Manz Crossmedia GmbH \& Co KG

Stolberggase 26, 1051 Wien 


\section{Vorwort}

Als Österreich Anfang 2009 für zwei Jahre in den Weltsicherheitsrat einzog, versprach es, dort als „ehrlicher Makler“ zur Zusammenarbeit im Interesse des Friedens beizutragen. Unser Land kündigte damals auch an, dass es sich in seiner Arbeit im Sicherheitsrat an den Grundprinzipien des Völkerrechts und der Rechtsstaatlichkeit orientieren werde. Ich selbst habe gegenüber der österreichischen Öffentlichkeit darüber hinaus betont, dass wir die österreichische Mitgliedschaft im Sicherheitsrat auch nützen wollen, um als „Bindeglied“ zur Europäischen Union der 27 zu fungieren und Österreich noch stärker als bisher zu einem Ort der Begegnung, einer „Drehscheibe für den Frieden“, zu machen.

Dieser Bericht soll darlegen, was Österreich in den vergangenen zwei Jahren unternommen hat, um diesen anspruchsvollen Vorgaben gerecht zu werden. In diesem Sinne sind insbesondere unsere Beiträge zur Tagesordnung des Sicherheitsrats während der beiden letzten Jahre - vom Gaza-Konflikt über das iranische Nuklearprogramm und die nordkoreanischen Raketentests bis hin zur Sudanfrage und zur jüngsten Krise in Côte d'Ivoire - detailliert dargestellt.

Wir wollten aber auch illustrieren, welche - über das intensive Tagesgeschehen hinausgehenden - inhaltlichen Akzente Österreich in seiner Arbeit im Sicherheitsrat gesetzt hat. Ich glaube, dass es uns in den vergangenen zwei Jahren insbesondere auch mit unseren Beiträgen zur Stärkung der Menschenrechte und der Rechtsstaatlichkeit, zum Schutz von Zivilisten (v.a. auch von Frauen und Kindern) in bewaffneten Konflikten sowie zum Kampf gegen die Straflosigkeit gelungen ist, im Sicherheitsrat ein Profil aufzubauen, das breite Anerkennung gefunden hat.

Es ist uns dabei, wie ich im Zuge meiner (insgesamt acht) Besuche in New York während der beiden vergangenen Jahre auch selbst feststellen konnte, durchwegs zugute gehalten worden, dass Österreich prinzipientreu, aber auch ausgewogen vorgegangen ist - dass wir uns z.B. für den Schutz der Zivilbevölkerung in Darfur und im Ostkongo genauso eingesetzt haben, wie in Gaza oder in Sri Lanka, und dass wir in jeder Konfliktsituation stets das Gespräch mit allen Streitparteien gesucht haben. Deshalb war es oft gerade auch Österreich, das dazu beitragen konnte, dass der Sicherheitsrat in besonders strittigen Fragen - wie etwa jener der menschlichen Dimension des Westsaharakonflikts - eine gemeinsame Sprache gefunden hat. 
Diese ausgleichende Rolle Österreichs hat wohl auch dazu beigetragen, dass Österreich während der letzten zwei Jahre mehrfach Ort wichtiger internationaler Begegnungen - wie der Westsaharagespräche in Dürnstein im August 2009 und der Wiener und Badener Sudangespräche vom Dezember 2010 gewesen ist. Im Zeichen unseres Engagements im Sicherheitsrat haben wir auch verbesserten Zugang zu Tätigkeitsfeldern im New Yorker VN-Kontext, wie z.B. zum VN-Peacebuilding, finden können. Angesichts meines prioritären Anliegens, Österreich als Sitz internationaler Organisationen zu stärken, freut es mich besonders, dass es in der Zeit unserer Sicherheitsratsmitgliedschaft u.a. zur Ansiedlung der Internationalen Anti-Korruptionsakademie in Laxenburg, des Wiener Zentrums für Abrüstung und Non-Proliferation und eines Büros des International Peace Institute in Wien gekommen ist.

Wir haben aber auch unser Vorhaben, im Sicherheitsrat als „Brücke“ zur EU der $27 \mathrm{zu}$ dienen, ernst genommen. Es war mir ein besonderes Anliegen, dass Österreich die jeweilige EU-Präsidentschaft und die EU-Institutionen in New York und Brüssel laufend mit Informationen aus dem Sicherheitsrat versorgt hat. Es ist auch auf Österreichs Initiative zurückzuführen, dass die Hohe Vertreterin der EU, Catherine Ashton, im Mai 2010 zum ersten Mal vor dem Sicherheitsrat sprechen konnte.

Während der 104 Wochen, die Österreich dem Sicherheitsrat angehört hat, haben unsere VertreterInnen an mehr als 600 Sitzungen des Sicherheitsrats teilgenommen und in tausenden Stunden an Verhandlungen über mehr als 250 formelle Beschlüsse des Sicherheitsrats (Resolutionen, Vorsitzerklärungen und Presseerklärungen) mitgewirkt.

Ich halte es aber auch für besonders wichtig, dass wir den „Globalisierungsschub“, den uns die Sicherheitsratsmitgliedschaft gebracht hat, gezielt für die außenpolitische Arbeit der kommenden Jahre nutzen. Die von Österreich angestrebte Mitgliedschaft im VN-Menschenrechtsrat in der Periode 20112014 stellt eine logische Fortsetzung unserer Sicherheitsratsaktivitäten dar. Ich will das Profil, das wir uns im Sicherheitsrat erworben haben, aber auch dafür nützen, um unser Engagement in den Themenkreisen „Rechtsstaatlichkeit“ und „Schutz von Zivilpersonen in Konfliktsituationen“ aktiv fortzusetzen und zu vertiefen.

Für unsere Wahl in den Sicherheitsrat war unser hohes Ansehen als langjähriger Truppensteller im Rahmen von friedenserhaltenden Operationen der Vereinten Nationen ein sehr gewichtiges Argument. Es ist daher aus meiner Sicht nur folgerichtig, wenn Österreich seine Aktivitäten im Rahmen des VN-Peacekeeping nachdrücklich fortführt. Als Mitglied des Sicherheitsrats hat Österreich aber auch einen vertieften Einblick in viele Aspekte des VNPeacebuilding erhalten. Auch hier dürften sich in den kommenden Jahren für Österreich neue Chancen für ein verstärktes außenpolitisches Engagement eröffnen. Dies habe ich etwa bei meiner Reise in den Sudan im Dezember 2010 sehr klar gesehen. 
Österreich hat seine Arbeit im Sicherheitsrat, wie ich schon früher betont habe, als Dienst an der Staatengemeinschaft gesehen - aber auch als Gelegenheit, auf internationaler Ebene in Bereichen, die uns besonders wichtig sind, österreichische Akzente zu setzen. Diese Bemühungen wollen wir jetzt, gestärkt durch die Erfahrung der letzten zwei Jahre, entschlossen fortführen. Abschließend möchte ich allen meinen Dank aussprechen, die zur erfolgreich verlaufenen dritten Mitgliedschaft Österreichs im Sicherheitsrat beigetragen haben: allen voran Botschafter Thomas Mayr-Harting und seinem Team in New York, aber auch dem Koordinationsteam in Wien unter der Leitung von Gesandtem Peter Huber sowie den vielen anderen MitarbeiterInnen in der Zentrale und an den Vertretungsbehörden, die täglich in die Arbeit im Sicherheitsrat eingebunden waren.

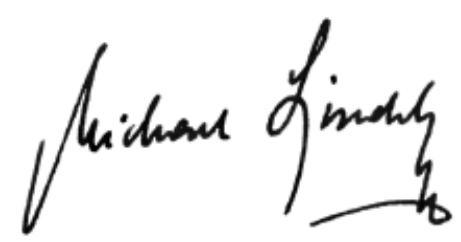

Dr. Michael Spindelegger

Bundesminister für europäische und internationale Angelegenheiten 



\section{Inhaltsverzeichnis}

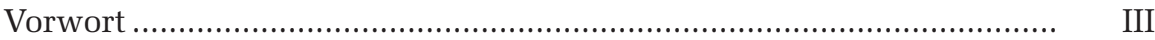

Abkürzungsverzeichnis.................................................................. XI

I. Einleitung .................................................................................. 1

II. Die Tätigkeit des Sicherheitsrats und die österreichische Mitwirkung - Geografische Themen ........ 11

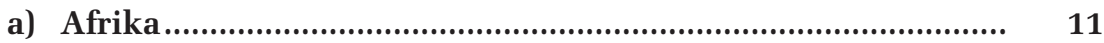

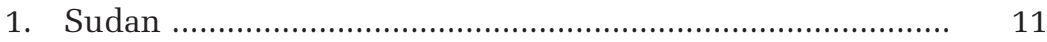

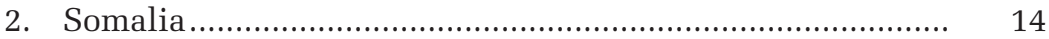

3. Demokratische Republik Kongo ......................................... $\quad 15$

4. Tschad und Zentralafrikanische Republik........................ 17

5. Côte d'Ivoire ................................................................... 18

6. Guinea-Bissau................................................................... 19

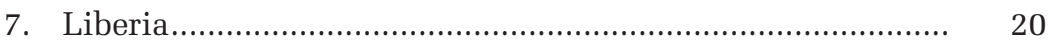

8. Sierra Leone....................................................................... 20

9. Burundi............................................................................. 21

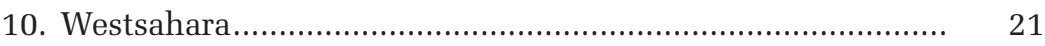

11. Regionale Themen ...................................................... 22

11.1. VN-Regionalbüro in Westafrika .............................. 22

11.2. Ad-Hoc Arbeitsgruppe für Konfliktprävention und -lösung in Afrika..................................................... 23

11.3. VN-Regionalbüro in Zentralafrika ............................. 23

11.4. Offene Debatte zum illegalen Klein- und Leichtwaffenhandel in Zentralafrika .......................................... 23

12. Sonstige Konflikte ........................................................... 24

12.1. Eritrea und Dschibuti ........................................... 24

12.2. Madagaskar ......................................................... 24

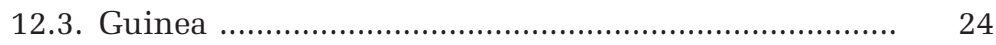

b) Naher und Mittlerer Osten .................................................. 25

1. Israel / Palästina .......................................................................... 25

2. Libanon........................................................................ 26

2.1. Sondertribunal ...................................................... 27

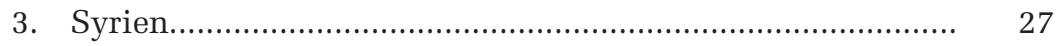

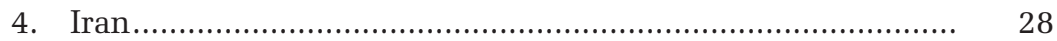

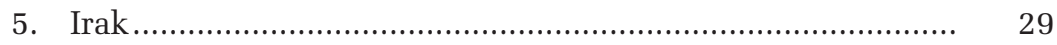




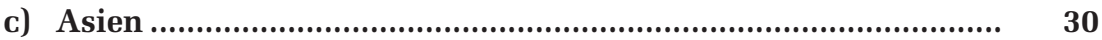

1. Demokratische Volksrepublik Korea ................................. $\quad 30$

2. Afghanistan .............................................................. 32

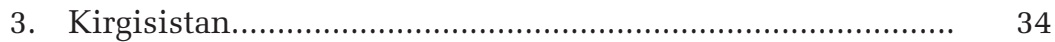

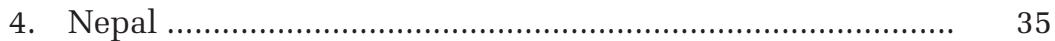

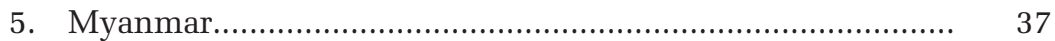

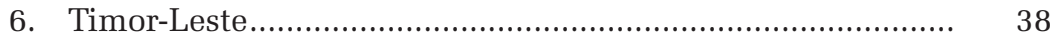

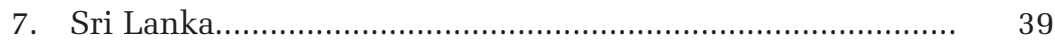

8. Pakistan ............................................................................. 41

9. Regionale Themen................................................................ 41

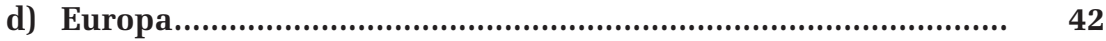

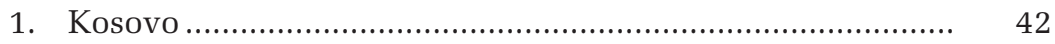

2. Bosnien und Herzegowina................................................. 43

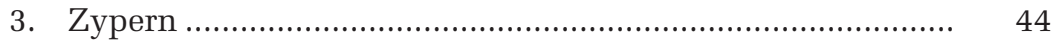

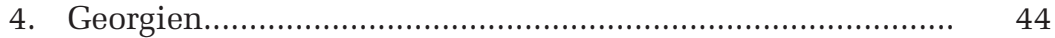

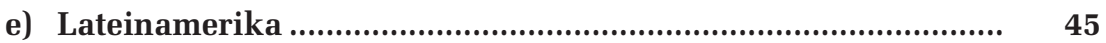

1. Haiti ................................................................................ 45

2. Sonstige Konflikte ......................................................... 47

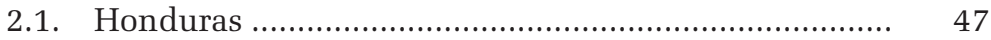

2.2. Venezuela und Kolumbien......................................... 48

2.3. Costa Rica und Nicaragua ...................................... 48

III. Die Tätigkeit des Sicherheitsrats und die österreichische Mitwirkung - Querschnittsthemen................................................ 49

1. Schutz der Zivilbevölkerung in bewaffneten Konflikten........... 49

2. Frauen, Frieden und Sicherheit - Bekämpfung sexueller Gewalt in Konflikten .............................................................. 51

3. Kinder und bewaffnete Konflikte ............................................. 53

4. Peacekeeping …..................................................................... 54

5. Peacebuilding ......................................................................... 56

6. Herrschaft des Rechts und Rechtsstaatlichkeit......................... 58

7. Internationale Tribunale und Gerichtshöfe.............................. 59

8. Wahrung des internationalen Friedens und der Sicherheit, Präventive Diplomatie und Mediation ........................................ $\quad 60$

9. Verbrechens- und Terrorismusbekämpfung ............................... 61

9.1. Antiterrorismuskomitee....................................................... 63

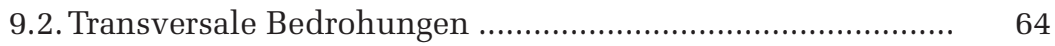

10. Nukleare Abrüstung und Non-Proliferation .............................. 65

10.1. 1540-Komitee................................................................. 66 
11. Arbeitsmethoden des Sicherheitsrats ....................................... 67

12. Zusammenarbeit der VN mit Regionalorganisationen ............... 68

IV. Der österreichische Vorsitz im Sicherheitsrat im November $2009 \quad 70$

1. Vorbereitungen ....................................................................... 71

2. Verhandlung und Annahme der SR-Resolution 1894 (2009) .... 72

V. EU-interne Zusammenarbeit und Stärkung der GASP ................... 73

VI. Koordinationsprozesse und Arbeitsabläufe.................................... $\quad 75$

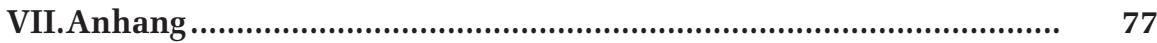

1. Zusammensetzung der österreichischen Delegation.................. 77

2. Österreichische Erklärungen im Sicherheitsrat und Ausgewählte Dokumente .............................................................. $\quad 77$

2.1. Ausgewählte österreichische Wortmeldungen.................... 77

2.2. Österreichische Votumserklärungen.................................. 112

2.3. Ausgewählte Resolutionen und Vorsitzerklärungen............ 119 



\section{Abkürzungsverzeichnis}

AL

AMISOM

ASG

AU

AU-PSC

BINUB

BINUCA

BMeiA

BMLVS

BNUB

BONUCA

CABSI

CEB

CNDP

CPA

CSTO
Arab League

Arabische Liga

African Union Mission in Somalia

Mission der Afrikanischen Union in Somalia

Assistant Secretary-General

Beigeordnete/r Generalsekretär/in

African Union

Afrikanische Union

Peace and Security Council of the African Union

Friedens- und Sicherheitsrat der Afrikanischen Union

Bureau intégré des Nations Unies au Burundi

Integriertes Büro der Vereinten Nationen in Burundi

Bureau intégré des Nations Unies pour la consolidation de la paix en République centrafricaine

Integriertes Büro der Vereinten Nationen für die Friedenskonsolidierung in der Zentralafrikanischen Republik

Bundesministerium für europäische und internationale Angelegenheiten

Bundesministerium für Landesverteidigung und Sport

Bureau des Nations Unies au Burundi

Büro der Vereinten Nationen in Burundi

Bureau des Nations Unies pour la consolidation de la paix en République centrafricaine

Büro der Vereinten Nationen zur Unterstützung der Friedenskonsolidierung in der Zentralafrikanischen Republik

Central Asia Border Security Initiative

Zentralasiatische Grenzsicherheitsinitiative

United Nations System Chief Executives Board for

Coordination

Koordinierungsrat der Leiter der Organisationen des

Systems der Vereinten Nationen

Congrès National pour la Défense du Peuple

Nationalkongress zur Verteidigung des Volkes [Demokratische Republik Kongo]

Comprehensive Peace Agreement

Umfassendes Friedensabkommen

Collective Security Treaty Organization

Organisation des Vertrags über Kollektive Sicherheit 


\section{Abkürzungsverzeichnis}

CTBT

CTC

CTED

CTITF

DFI

E3+3

ECCAS

ECOSOC

ECOWAS

ERSG

EU

EUNAVFOR

EUFOR

EUFOR Tchad/ RCA

EU-HV

EULEX

FARDC
Comprehensive Nuclear-Test-Ban Treaty

Vertrag über das umfassende Verbot von Nuklearversuchen

Counter-Terrorism Committee

Komitee zur Bekämpfung des Terrorismus

Counter-Terrorism Committee Executive Directorate Exekutivdirektorium des Komitees zur Bekämpfung des Terrorismus

Counter-Terrorism Implementation Task Force

Arbeitsstab Terrorismusbekämpfung

Development Fund for Iraq

Entwicklungsfonds für Irak

Internationales Verhandlungsformat zu Iran bestehend aus Deutschland, Frankreich, Großbritannien sowie Russischer Föderation, Vereinigten Staaten von Amerika und Volksrepublik China

Economic Community of Central African States

Wirtschaftsgemeinschaft Zentralafrikanischer Staaten

Economic and Social Council

Wirtschafts- und Sozialrat

Economic Community of West African States

Wirtschaftsgemeinschaft der westafrikanischen Staaten

Executive Representative of the Secretary-General

Exekutivbeauftragte/r des Generalsekretärs

Europäische Union

European Union Naval Forces

Seestreitkräfte der Europäischen Union

European Union Force

Einsatzkräfte der Europäischen Union

Opération militaire de l’Union européenne en République du Tchad et en République centrafricaine

Militärische Operation der Europäischen Union in der

Republik Tschad und in der Zentralafrikanischen

Republik

Hohe Vertreterin der Europäischen Union für Außen- und Sicherheitspolitik

European Union Rule of Law Mission in Kosovo Rechtsstaatlichkeitsmission der Europäischen Union im Kosovo

Forces Armées de la République démocratique du Congo Streitkräfte der Demokratischen Republik Kongo 
FDLR

FEO

FMCT

GASP

IACA

IAEO

ICTR

ICTY

IPI

ISAF

IStGH

JEM

KLW

LISD

LJM

LRA

LTTE

MINURCAT
Forces démocratiques pour la libération du Rwanda Demokratische Kräfte für die Befreiung Ruandas

Friedenserhaltende Operation(en)

Treaty banning the production of fissile material for nuclear weapons or other nuclear explosive devices Vertrag über das Verbot der Herstellung von spaltbarem Material für Kernwaffen oder andere Kernsprengkörper

Gemeinsame Außen- und Sicherheitspolitik

International Anti-Corruption Academy

Internationale Anti-Korruptionsakademie

Internationale Atomenergie-Organisation

International Criminal Tribunal for Rwanda

Internationaler Strafgerichtshof für Ruanda

International Criminal Tribunal for the Former Yugoslavia Internationaler Strafgerichtshof für das ehemalige Jugoslawien

International Peace Institute

Internationales Friedensinstitut

International Security Assistance Force

Internationale Sicherheitsbeistandstruppe

Internationaler Strafgerichtshof

Justice and Equality Movement

Bewegung für Gerechtigkeit und Gleichheit [Sudan]

Kleinwaffen und leichte Waffen

Liechtenstein Institute on Self-Determination at Princeton University

Liechtenstein-Institut für Selbstbestimmung an der Princeton Universität

Liberation and Justice Movement

Bewegung für Befreiung und Gerechtigkeit [Sudan]

Lord's Resistance Army

Widerstandsarmee des Herrn [Region Zentralafrika]

Liberation Tigers of Tamil Eelam

Befreiungstiger von Tamil Eelam [Sri Lanka]

Mission des Nations Unies en République centrafricaine et au Tchad

Mission der Vereinten Nationen in der Zentralafrikanischen Republik und im Tschad 
MINURSO Mission des Nations Unies pour l'organisation d'un référendum au Sahara occidental

Mission der Vereinten Nationen für das Referendum in Westsahara

MINUSTAH Mission des Nations Unies pour la stabilisation en Haiti Stabilisierungsmission der Vereinten Nationen in Haiti

MONUC Mission de l'Organisation des Nations Unies en République démocratique du Congo

Mission der Organisation der Vereinten Nationen in der Demokratischen Republik Kongo

MONUSCO Mission de l'Organisation des Nations Unies pour la stabilisation en République démocratique du Congo Stabilisierungsmission der Organisation der Vereinten Nationen in der Demokratischen Republik Kongo

NATO North Atlantic Treaty Organisation Nordatlantikvertrags-Organisation

NCP

National Congress Party

Nationale Kongresspartei [Sudan]

NPT

NRO

Treaty on the Non-Proliferation of Nuclear Weapons Vertrag über die Nichtverbreitung von Kernwaffen

OAS

Nicht-Regierungsorganisation(en)

Organization of American States

Organisation Amerikanischer Staaten

OCHA

Office for the Coordination of Humanitarian Affairs Büro für die Koordinierung humanitärer Angelegenheiten

OHCHR Office of the High Commissioner for Human Rights Büro der Hochkommissarin für Menschenrechte

OSZE

ÖV

$\mathrm{P}-5$

Organisation für Sicherheit und Zusammenarbeit in Europa

Österreichische Vertretung

Ständige Mitglieder des Sicherheitsrats der Vereinten Nationen (Frankreich, Großbritannien, Russische Föderation, Vereinigte Staaten von Amerika, Volksrepublik China)

PBC Peacebuilding Commission

Kommission für Friedenskonsolidierung

PRST Presidential Statement

Vorsitzerklärung

RSG Representative of the Secretary-General

Beauftragte/r des Generalsekretärs 


\begin{tabular}{|c|c|}
\hline SA & $\begin{array}{l}\text { Special Adviser } \\
\text { Sonderberater/in }\end{array}$ \\
\hline SE & $\begin{array}{l}\text { Special Envoy } \\
\text { Sondergesandte/r }\end{array}$ \\
\hline SLA & $\begin{array}{l}\text { Sudan Liberation Army } \\
\text { Befreiungsarmee Sudans }\end{array}$ \\
\hline SPLM & $\begin{array}{l}\text { Sudan Peoples' Liberation Movement } \\
\text { Sudanesische Volksbefreiungsbewegung }\end{array}$ \\
\hline SRSG & $\begin{array}{l}\text { Special Representative of the Secretary-General } \\
\text { Sonderbeauftragte/r des Generalsekretärs }\end{array}$ \\
\hline UGS & Untergeneralsekretär/in \\
\hline UNAMA & $\begin{array}{l}\text { United Nations Assistance Mission in Afghanistan } \\
\text { Unterstützungsmission der Vereinten Nationen in Afgha- } \\
\text { nistan }\end{array}$ \\
\hline UNAMI & $\begin{array}{l}\text { United Nations Assistance Mission for Iraq } \\
\text { Unterstützungsmission der Vereinten Nationen für den } \\
\text { Irak }\end{array}$ \\
\hline UNAMID & $\begin{array}{l}\text { African Union-United Nations Hybrid Operation in Darfur } \\
\text { Hybrider Einsatz der Afrikanischen Union und der } \\
\text { Vereinten Nationen in Darfur }\end{array}$ \\
\hline UNCC & $\begin{array}{l}\text { United Nations Compensation Commission } \\
\text { Entschädigungskommission der Vereinten Nationen }\end{array}$ \\
\hline UNDOF & $\begin{array}{l}\text { United Nations Disengagement Observer Force } \\
\text { Beobachtertruppe der Vereinten Nationen für die } \\
\text { Truppenentflechtung }\end{array}$ \\
\hline
\end{tabular}

UNFFM United Nations Fact Finding Mission on the Gaza Conflict Ermittlungsmission der Vereinten Nationen für den Gaza Konflikt

UNFICYP United Nations Peacekeeping Force in Cyprus Friedenstruppe der Vereinten Nationen in Zypern

UNIDO United Nations Industrial Development Organization Organisation der Vereinten Nationen für industrielle Entwicklung

UNIFIL United Nations Interim Force in Lebanon Interimstruppe der Vereinten Nationen im Libanon

UNIOGBIS United Nations Integrated Peacebuilding Office in GuineaBissau Integriertes Büro der Vereinten Nationen für die Friedenskonsolidierung in Guinea-Bissau 
UNIPSIL United Nations Integrated Peacebuilding Office in Sierra Leone

Integriertes Büro der Vereinten Nationen für die Friedenskonsolidierung in Sierra Leone

UNMIK

United Nations Interim Administration Mission in Kosovo Übergangsverwaltungsmission der Vereinten Nationen im Kosovo

UNMIL United Nations Mission in Liberia

Mission der Vereinten Nationen in Liberia

UNMIN United Nations Mission in Nepal

Mission der Vereinten Nationen in Nepal

UNMIS United Nations Mission in the Sudan

Mission der Vereinten Nationen im Sudan

UNMIT United Nations Integrated Mission in Timor-Leste Integrierte Mission der Vereinten Nationen in Timor-Leste

UNOCA United Nations Office for Central Africa Büro der Vereinten Nationen für Zentralafrika

UNOCI United Nations Operation in Côte d'Ivoire Operation der Vereinten Nationen in Côte d'Ivoire

UNODA United Nations Office for Disarmament Affairs Büro der Vereinten Nationen für Abrüstungsfragen

UNODC United Nations Office on Drugs and Crime Büro der Vereinten Nationen für Drogen- und Verbrechensbekämpfung

UNOGBIS United Nations Peacebuilding Support Office in GuineaBissau

Büro der Vereinten Nationen zur Unterstützung der Friedenskonsolidierung in Guinea-Bissau

UNOMIG United Nations Observer Mission in Georgia Beobachtermission der Vereinten Nationen in Georgien

UNOWA United Nations Office for West Africa Büro der Vereinten Nationen für Westafrika

UNRCCA United Nations Regional Centre for Preventive Diplomacy for Central Asia

Regionalzentrum der Vereinten Nationen für vorbeugende Diplomatie für Zentralasien

UNRWA United Nations Relief and Works Agency for Palestine Refugees in the Near East

Hilfswerk der Vereinten Nationen für Palästinaflüchtlinge im Nahen Osten 
UNTOC United Nations Convention against Transnational Organized Crime

Konvention der Vereinten Nationen gegen die grenzüberschreitende organisierte Kriminalität

UNTSO United Nations Truce Supervision Organization Organisation der Vereinten Nationen zur Überwachung des Waffenstillstands

$\mathrm{VN}$

Vereinte Nationen

VN-GS

Generalsekretär der Vereinten Nationen

VN-GV

Generalversammlung der Vereinten Nationen

VN-SR

Sicherheitsrat der Vereinten Nationen 



\section{Einleitung}

Österreich war von 1. Jänner 2009 bis 31. Dezember 2010 - nach 1973/74 und 1991/92 - zum dritten Mal Mitglied des Sicherheitsrats der Vereinten Nationen (VN-SR). Zum ersten Mal jedoch musste sich Österreich einer umkämpften Abstimmung stellen und konnte bereits im ersten Wahlgang die notwendige Zweidrittelmehrheit in der VN-Generalversammlung (VN-GV) erreichen. Dies stellte einen besonderen Vertrauensbeweis der internationalen Staatengemeinschaft dar. Neben den fünf ständigen Mitgliedern (P-5) Frankreich, Großbritannien, der Russischen Föderation, den Vereinigten Staaten von Amerika und der Volksrepublik China, gehörten dem VN-SR während dieses Zeitraums - abgesehen von Österreich - auch Japan, Mexiko, die Türkei und Uganda als nicht-ständige Mitglieder an. Im Jahr 2009 saß Österreich überdies mit Burkina Faso, Costa Rica, Kroatien, Libyen und Vietnam (deren Funktionsperiode schon Anfang 2008 begonnen hatte) am Tisch des VN-SR. An die Stelle dieser fünf Staaten traten im zweiten Jahr unserer SR-Mitgliedschaft Bosnien und Herzegowina, Brasilien, Gabun, Libanon und Nigeria (als gewählte Mitglieder für die Periode 2010/2011).

Insgesamt hat der VN-SR in den zwei Jahren der österreichischen Mitgliedschaft 403 formelle Sitzungen und 212 informelle Konsultationen abgehalten, bei denen neben aktuellen Krisen Entwicklungen in sämtlichen Ländern, in denen die Vereinten Nationen (VN) mit Friedensmissionen bzw. durch Sonderbeauftragte oder sonstige VertreterInnen des Generalsekretärs der VN (VN-GS) engagiert sind, sowie diverse Querschnittsthemen erörtert wurden. Auf Basis dieser Beratungen hat der VN-SR in den Jahren 2009 und 2010107 Resolutionen, 65 Vorsitzerklärungen und 88 Presseerklärungen beschlossen. Darüber hinaus fanden regelmäßig Sitzungen der vom VN-SR eingesetzten 19 Sanktionenkomitees und Arbeitsgruppen statt.

Als Mitglied des VN-SR war Österreich von Anbeginn mit zentralen Herausforderungen des internationalen Krisenmanagements konfrontiert. Schon die allerersten Tage standen im Zeichen der Kampfhandlungen in und um den Gazastreifen. Bereits am sechsten Tag der österreichischen SR-Mitgliedschaft nahm Bundesminister Michael Spindelegger, der im Laufe der folgenden zwei Jahre noch sieben weitere Male nach New York kommen sollte, an einer Krisensitzung des VN-SR teil. Österreich setzte sich dabei tatkräftig für einen möglichst breiten Konsens unter den 15 SR-Mitgliedern für einen Resolutionsentwurf ein, in dem ein sofortiges Ende der Gewalthandlungen und Zugang für humanitäre Hilfe im Gazastreifen gefordert wurde. Die SRResolution 1860 (2009) wurde am 8. Jänner 2009 - nach intensiven Verhandlungen - mit 14 Ja-Stimmen und einer Enthaltung (Vereinigte Staaten von Amerika) angenommen.

Die Annahme der SR-Resolution 1860 (2009) bot Österreich im Übrigen auch eine erste Möglichkeit, in einer Votumserklärung jenes Thema anzusprechen, das in der Folge zu einem der Themenschwerpunkte der österreichi- 


\section{Einleitung}

schen SR-Mitgliedschaft werden sollte: die unbedingte Verpflichtung aller Parteien eines Konflikts, das humanitäre Völkerrecht einzuhalten.

Die dringende Notwendigkeit, diese Frage erneut zu thematisieren, ergab sich aus österreichischer Sicht schon wenige Monate später - angesichts der Notlage hunderttausender Zivilisten, die im Zuge der schweren bewaffneten Auseinandersetzungen zwischen der Regierung von Sri Lanka und der Terrororganisation „Tamil Tigers“ (Befreiungstiger von Tamil Eelam, LTTE) zwischen die Frontlinien geraten waren. Neben VN-GS Ban Ki-moon waren es damals v.a. die nicht-ständigen SR-Mitglieder Mexiko, Costa Rica und Österreich sowie Frankreich und Großbritannien, die den VN-SR drängten, sich mit dieser Frage zu befassen, was schließlich auch gelang. Am 11. Mai 2009 gaben die Außenminister Frankreichs, Großbritanniens und Österreichs, Kouchner, Miliband und Spindelegger am Rande einer SR-Debatte zum Nahen Osten in New York eine gemeinsame Pressekonferenz zur Lage in Sri Lanka, in der sie die Verantwortung der srilankischen Regierung für den Schutz ihrer Bevölkerung unterstrichen, den Zugang unabhängiger Beobachter zu den Flüchtlingslagern forderten und auf eine politische Lösung des Konflikts drängten. Zwei Tage später einigte sich der VN-SR auf gemeinsame Initiative Frankreichs, Großbritanniens und Österreichs erstmals auf eine formelle Presseerklärung zu Sri Lanka (SC/9659), in der alle Parteien dringend zur Einhaltung des humanitären Völkerrechts angehalten, die LTTE zur Niederlegung der Waffen und die srilankische Regierung zur Erleichterung des humanitären Zugangs aufgerufen wurden. Trotz der Fortsetzung der militärischen Operation durch die Regierung von Sri Lanka gehen die meisten Beobachter davon aus, dass die srilankischen Streitkräfte angesichts der Diskussionen im VN-SR (und sonstiger VN-Aktivitäten) auf die Sicherheitsbedürfnisse der Zivilisten im Konfliktgebiet größere Rücksicht genommen haben, als dies sonst der Fall gewesen wäre.

Der Schutz von Zivilisten in Konfliktsituationen stand auch im Mittelpunkt des österreichischen SR-Vorsitzes im Monat November 2009. Am 11. November 2009 fand zu diesem Thema unter dem Vorsitz von Bundesminister Michael Spindelegger eine hochrangig besetzte Debatte statt, in deren Verlauf über 60 Staaten das Wort ergriffen. Unter den DebattenteilnehmerInnen befand sich - über österreichische Initiative - auch die stellvertretende VNHochkommissarin für Menschenrechte, Kang Kyung-wha. Damit erhielt das Hochkommissariat zum ersten Mal seit 2005 eine Möglichkeit, vor dem VN-SR zu sprechen. Der VN-SR nahm in dieser Debatte einstimmig die - von Österreich initiierte und verhandelte - SR-Resolution 1894 (2009) an. Mit dieser (inzwischen als „landmark document“ anerkannten) Entschließung soll neben einem besseren Zugang für humanitäre Hilfsorganisationen und dem Kampf gegen die Straflosigkeit insbesondere auch sichergestellt werden, dass die VN im Rahmen ihrer eigenen friedenserhaltenden Operationen (FEO) selbst zu einem verbesserten Schutz von Zivilpersonen im jeweiligen Einsatzgebiet beitragen. 
Während des österreichischen SR-Vorsitzes im November 2009 standen auch Debatten zum Irak, zu Bosnien und Herzegowina, zu Somalia, zur Demokratischen Republik Kongo, zum Sudan und zum Nahen Osten auf der Tagesordnung des VN-SR. Weiters kam es - nach der Unterzeichnung eines Militärabkommens zwischen den Vereinigten Staaten von Amerika und Kolumbien - zu starken Spannungen zwischen Venezuela und Kolumbien. Beide Staaten befassten in dieser Situation den österreichischen SR-Vorsitz, der in informellen Gesprächen zur Zufriedenheit beider Seiten zur Deeskalation beitragen konnte.

Über die von Österreich verhandelte SR-Resolution 1894 (2009) zum Schutz der Zivilbevölkerung in bewaffneten Konflikten hinaus hat Österreich auch nach seinem Vorsitzmonat systematisch über deren Einhaltung im Falle konkreter VN-Einsätze gewacht - ein erstes Mal schon im Dezember 2009 aus Anlass der Annahme der SR-Resolution 1906 (2009) zur Verlängerung der VN-Mission in der Demokratischen Republik Kongo (MONUC). Dort hatten sich - neben den Rebellengruppen - regelmäßig auch Angehörige der kongolesischen Streitkräfte (FARDC) schwerer Übergriffe gegen die Zivilbevölkerung, v.a. auch sexueller Gewaltakte gegen Frauen, schuldig gemacht. Gemeinsam mit Costa Rica konnte Österreich in SR-Resolution 1906 (2009) die Verankerung der sog. „conditionality policy“ erreichen, die den VN die Verpflichtung auferlegt, den FARDC die Unterstützung zu entziehen, wenn die Gefahr grober Menschenrechtsverletzungen besteht.

Ein Land, in dem die Frage des Schutzes der Zivilisten im Jahr 2010 in den Arbeiten des VN-SR ebenfalls eine sehr wesentliche Rolle spielte, war der Tschad. Das Mandat der VN-Mission in der Zentralafrikanischen Republik und im Tschad (MINURCAT) konzentrierte sich von Beginn an auf den Schutz der Zivilbevölkerung in den Flüchtlingslagern. MINURCAT wurde nach dem Auslaufen der EU-Überbrückungsoperation EUFOR Tchad/RCA im März 2009 um eine militärische Komponente erweitert, an der sich bis Dezember 2009 auch Österreich beteiligte. Anfang 2010 teilte die Regierung des Tschad den VN allerdings mit, dass sie einer Verlängerung des MINURCATMandats nicht mehr zustimmen wolle. Nach schwierigen Verhandlungen wurde mit SR-Resolution 1923 (2010) im Mai 2010 doch noch eine Einigung über die Verlängerung des MINURCAT-Mandats bis Ende 2010 erreicht; die verkleinerte militärische Komponente der Mission war aber nur mehr für den Schutz der zivilen VN-Präsenz verantwortlich. Auf österreichisches Drängen wurde in die Resolution allerdings eine Notfallsklausel aufgenommen, die MINURCAT autorisierte, auf Akte der Gewalt gegen Zivilisten im unmittelbaren Umfeld der Mission zu reagieren. Außerdem schreibt SRResolution 1923 (2010) in sehr klarer Form die Verantwortung der tschadischen Regierung für den Schutz der Zivilisten fest; die seither feststellbaren Bemühungen des Tschad zum Aufbau der für diese Schutzaufgaben zuständigen Polizeikräfte sind zweifelsohne auch auf die im VN-SR stattgefundenen Beratungen zurückzuführen. 


\section{Einleitung}

Die Debatten im VN-SR haben im Übrigen auch eine breite Diskussion zum Grundsatz der Zustimmung des Gastlandes (,host country consent“) ausgelöst: Es wächst im VN-SR zunehmend die Überzeugung, dass Länder, in denen VN-Missionen zum Einsatz kommen, mit den VN eigentlich eine Vereinbarung (,compact“) zur Bewältigung einer bestimmten Aufgabe eingehen, und es daher dem Geist dieser Abmachung widerspräche, die Zustimmung zum Verbleib der jeweiligen VN-Mission einseitig zurückzuziehen, bevor die gesetzte Aufgabe erfüllt ist.

Dies war dann auch der Ansatz, mit dem die große Mehrzahl der SR-Mitglieder an die Forderung von Präsident Joseph Kabila nach einem schrittweisen Abzug der gesamten VN-Mission MONUC heranging. Der Großteil der Mitglieder des VN-SR war sich einig, dass ein frühzeitiger Rückzug dieser - mit 20.000 Mann noch immer weltweit größten - FEO der VN v.a. im Ostkongo mit katastrophalen Folgen für die Zivilbevölkerung verbunden wäre. Dieser Standpunkt wurde mit Präsident Kabila - im Rahmen einer Mission des VN-SR in die Demokratische Republik Kongo im Mai 2010 - auch direkt erörtert. Die VN-Mission wurde im Mai 2010 vorerst bis Mitte 2011 verlängert.

Auf der Agenda des VN-SR haben afrikanische Themen traditionell ein deutliches Übergewicht. Dies war auch in der Zeit der österreichischen SR-Mitgliedschaft der Fall. Der VN-SR befasste sich im Berichtszeitraum insbesondere mit Fragen des Konfliktmanagements in der Region der Großen Seen, in Westafrika, im Sudan und am Horn von Afrika. Die vergangenen zwei Jahre waren durch eine wachsende Zusammenarbeit zwischen den VN, der Afrikanischen Union (AU) und afrikanischen Regionalorganisationen wie der Wirtschaftsgemeinschaft der westafrikanischen Staaten (ECOWAS) gekennzeichnet, zuletzt v.a. auch in Côte d'Ivoire. Während der österreichischen SR-Mitgliedschaft ist der VN-SR auch dreimal nach Afrika gereist und hat bei diesen Gelegenheiten u.a. das Hauptquartier der AU in Addis Abeba, Ruanda, die Demokratische Republik Kongo (zweimal), Liberia und den Sudan besucht.

Österreich hat sich in seiner SR-Arbeit zu Afrika, wie schon dargestellt, insbesondere auf seinen Themenschwerpunkt „Schutz von Zivilisten“ konzentriert. Darüber hinaus ergaben sich zahlreiche Möglichkeiten, die Arbeit der Wiener VN-Einrichtungen, insbesondere den Einsatz des VN-Büros zur Drogen- und Verbrechensbekämpfung (UNODC) im Kampf gegen den Drogenhandel in Westafrika, zu thematisieren. Der Beitrag und die Verantwortung betroffener Staaten wurden von Österreich in allen afrikanischen Angelegenheiten unterstrichen, was von unseren afrikanischen Partnern im VN-SR und den jeweiligen Sonderbeauftragten des VN-GS (SRSG) geschätzt wurde.

Ganz allgemein hat die SR-Arbeit zu Afrika auf afrikanischer Seite zweifelsohne zu einer deutlichen Erhöhung der Visibilität Österreichs geführt. Ein konkretes Ergebnis dieses Engagements war die Aufnahme Österreichs in die länderspezifische Konfiguration der VN-Kommission für Friedenskonsolidierung (PBC) für Sierra Leone. Aktiv eingebracht hat sich Österreich auch in Debatten des VN-SR zum Thema Sudan. Dieses österreichische Engagement 
wurde durch den Umstand erleichtert, dass Österreich sowohl in Khartum als auch im Südsudan als objektiver und historisch unbelasteter Partner angesehen wird. Das österreichische Angebot, beiden Seiten für den Fall einer Sezession des Südens Expertise in Fragen des Staatsbürgerschaftsrechts zur Verfügung zu stellen, stieß im Norden und im Süden auf eine grundsätzlich positive Reaktion. Im November 2010 hat in Wien und Baden - über Initiative des Österreichischen Instituts für Europäische Sicherheitspolitik und der Politischen Akademie - eine Sudan-Tagung mit hochrangigen Teilnehmern aus dem Norden und dem Süden stattgefunden. Bundesminister Michael Spindelegger ist allein 2010 insgesamt siebenmal mit seinem sudanesischen Amtskollegen Ali Karti zusammengetroffen und hat im Dezember 2010 Khartum und Juba (Südsudan) besucht.

Ein afrikanisches Thema, mit dem Österreich während seiner SR-Mitgliedschaft wiederholt konfrontiert wurde, war die Westsahara. In der eigentlichen Kernfrage, dem künftigen Status dieses Territoriums, gab es im Berichtszeitraum keine Bewegung. Konkret ging es in den SR-Konsultationen zur Westsahara aber auch darum, ob das Mandat der Mission der Vereinten Nationen für das Referendum in Westsahara (MINURSO) mit einer Menschenrechtskomponente versehen werden könne, wie dies in den Mandaten jüngerer VN-Missionen inzwischen Standard ist. Dies erwies sich allerdings nicht als konsensfähig. Über österreichischen Vorschlag wurde im April 2009 nach schwierigen Verhandlungen aber erstmals Einigung erzielt, in SR-Resolution 1871 (2009) zumindest einen allgemeinen Hinweis auf die „menschliche Dimension“ des Konflikts aufzunehmen.

Im August 2009 trafen die Konfliktparteien über Einladung Österreichs in Dürnstein zu informellen Gesprächen unter der Führung des Persönlichen Gesandten des VN-GS Christopher Ross zusammen. Diese sind aus Sicht der Beteiligten atmosphärisch gut verlaufen. Im März, November und Dezember 2010 fanden in der Nähe von New York drei weitere Gesprächsrunden statt. Die SR-Konsultationen, die im November 2010 (auch über Ersuchen Österreichs) wegen der Gewaltakte rund um die Räumung des sahrauischen Zeltlagers bei El Aaiun stattfanden, haben die Dringlichkeit einer Bewegung in den Bemühungen zur Bewältigung dieses ungelösten Konflikts - einschließlich eines substantiellen Dialogs der Parteien zu humanitären Themen - neuerlich unterstrichen.

Ein zentrales - und aus österreichischer Sicht besonders wichtiges - Thema, dem sich der VN-SR in den vergangenen zwei Jahren widmete, war der Kampf gegen die nukleare Proliferation. Österreich hat diese Bemühungen - im Hinblick auf seine klare Grundsatzposition in dieser Frage und als Sitzstaat der Internationalen Atomenergie-Organisation (IAEO) - mit Nachdruck und aus Überzeugung unterstützt.

Im Juni 2009 erweiterte der VN-SR in Reaktion auf mehrere Raketentests der Demokratischen Volksrepublik Korea mit SR-Resolution 1874 (2009) ein- 
stimmig das bestehende Sanktionenregime, das nunmehr auch die Möglichkeit von Schiffsfrachtinspektionen vorsieht.

Am 24. September 2009 fand zum Thema nukleare Abrüstung und Non-Proliferation ein Gipfeltreffen des VN-SR unter dem Vorsitz von US-Präsident Barack Obama statt, bei dem Österreich durch Bundespräsident Heinz Fischer und Bundesminister Michael Spindelegger vertreten war. In der einstimmig angenommenen SR-Resolution 1887 (2009) bekannte sich der VN-SR erstmals zum Ziel einer Welt ohne Nuklearwaffen.

Im Juni 2010 reagierte der VN-SR auf das fortgesetzte iranische Nuklearprogramm - und die Entdeckung einer geheimen nuklearen Anreicherungsanlage in Qom - nach schwierigen Verhandlungen der „E3+3“ (Deutschland, Frankreich, Großbritannien sowie Russische Föderation, Vereinigte Staaten von Amerika und Volksrepublik China) mit einer deutlichen Ausweitung und Verschärfung des bestehenden Sanktionenregimes. SR-Resolution 1929 (2010), die mit zwölf Ja-Stimmen gegen zwei Nein-Stimmen (Brasilien, Türkei) bei einer Enthaltung (Libanon) angenommen wurde, enthält u.a. ein Verbot von Raketentests, ein weitgehendes Waffenembargo und Beschränkungen für den iranischen Transport-, Banken- und Finanzdienstleistungssektor.

Ein Fragenkreis, mit dem sich der VN-SR während der letzten zwei Jahre wiederholt beschäftigte, war die notwendige Reform der FEO der VN. Innerhalb der letzten zehn Jahre hat sich die Zahl der an VN-Einsätzen beteiligten Militär-, Polizei- und Zivilkräfte auf rund 125.000 versechsfacht. Das Aufgabenspektrum dieser Missionen hat sich ebenfalls deutlich erweitert. An die Stelle klassischer Missionen zur Überwachung von Waffenstillstandslinien sind zunehmend Einsätze in Regionen getreten, die von innerstaatlichen Konflikten betroffen sind, mit Mandaten, die neben dem Schutz der Zivilbevölkerung auch Aufgaben wie die Stärkung der Rechtsstaatlichkeit, den Schutz der Menschenrechte und die Reform des Sicherheitssektors beinhalten. Die zur operativen Führung von VN-Einsätzen vorgesehenen Strukturen und Verfahren sind bisher aber im Wesentlichen unverändert geblieben.

Vor diesem Hintergrund hatten Großbritannien und Frankreich schon Anfang 2009 im VN-SR einen Reflexionsprozess eingeleitet, in den sich auch Österreich als traditioneller VN-Truppensteller aktiv eingebracht hat. Als ein erstes Ergebnis dieser Diskussionen wurden Bemühungen unternommen, den Informationsfluss zwischen den einzelnen VN-Missionen und dem VNSekretariat in New York zu verbessern. Der VN-SR hat den Dialog mit den Truppenstellern, insbesondere vor der Beschlussfassung über Mandate, intensiviert. Zu den allgemeinen SR-Debatten über die Zukunft des VNPeacekeeping (jeweils im August 2009 bzw. 2010) wurden erstmals auch die Truppenkommandanten aller VN-Missionen eingeladen, darunter 2009 auch Generalmajor Wolfgang Jilke als damaliger Kommandant von UNDOF. 
Im Verlauf der vergangenen zwei Jahre hat sich Österreich im VN-SR auch systematisch und gezielt für den Schutz von Frauen und Kindern in Konfliktsituationen eingesetzt.

Die Arbeitsgruppe des VN-SR zu Kindern und bewaffneten Konflikten stand beide Jahre unter der Leitung Mexikos, das im August 2009 mit der Annahme von SR-Resolution 1882 (2009) einen bedeutenden Fortschritt erzielen konnte: Gemäß dieser Resolution werden seither nicht nur jene Konfliktparteien, die sich der Rekrutierung von Kindersoldaten schuldig machen, in „schwarze Listen“ des VN-GS aufgenommen, sondern auch solche, die Kinder im Kontext eines bewaffneten Konflikts töten, verstümmeln oder sexueller Gewalt aussetzen.

Zum Themenkreis „Frauen, Frieden und Sicherheit“ wurden während der österreichischen SR-Mitgliedschaft drei Resolutionen angenommen: SRResolution 1888 (vom September 2009), mit der die Funktion eines/einer Sonderbeauftragten (SRSG) zu sexueller Gewalt in Konflikten geschaffen wurde, SR-Resolution 1889 (vom Oktober 2009) über die Rolle von Frauen in Peacebuilding und Wiederaufbau sowie SR-Resolution 1960 (vom Dezember 2010), mit der der VN-GS aufgefordert wird, seinen jährlichen Berichten an den VN-SR zu sexueller Gewalt in bewaffneten Konflikten Listen von Gruppierungen beizuschließen, die solche Gewaltakte begehen, und die entsprechenden „Monitoring, Analysis and Reporting Arrangements“ zu schaffen.

Österreich hat an der Erarbeitung all dieser Resolutionen aktiv mitgewirkt. Darüber hinaus hat Österreich viele Monate sehr intensiv an der Vorbereitung des - am 26. Oktober 2010 begangenen - 10. Jahrestages der Annahme der SR-Resolution 1325 (2000) („,Frauen, Frieden und Sicherheit“) mitgewirkt. Die wichtigsten Etappen dieses Vorbereitungsprozesses waren der österreichische VN-Retreat in Alpbach Anfang September 2010, eine (vom kanadischen Außenminister Lawrence Cannon im Zusammenwirken mit Bundesminister Michael Spindelegger organisierte) „Commitment Conference" am Rande der VN-GV Ende September 2010 in New York und ein (im Oktober 2010 von Österreich, Mexiko und Großbritannien gemeinsam veranstaltetes) öffentliches Treffen von SR-Mitgliedern mit Vertretern der Zivilgesellschaft.

Auf diesen Arbeiten aufbauend, konnte der VN-SR in seiner öffentlichen Sitzung vom 26. Oktober 2010, an der u.a. US-Außenministerin Hillary Clinton und Bundesminister Michael Spindelegger teilnahmen, eine Vorsitzerklärung (S/PRST/2010/22) annehmen, in der sich der VN-SR für die Verwendung von 26 Indikatoren ausspricht, mit denen Fortschritte bei der Umsetzung von SR-Resolution 1325 (2000) künftig messbar gemacht werden sollen. Insgesamt hat es nach Meinung der meisten Beobachter in den zehn Jahren seit der Annahme der SR-Resolution 1325 (2000) zum Themenbereich „Frauen, Frieden und Sicherheit“ nie so viele konkrete Entwicklungen gegeben wie in den beiden letzten Jahren; ein Ergebnis, zu dem Österreich nach allgemeiner Einschätzung maßgeblich beigetragen hat. 
Eine gute Möglichkeit zu gestaltender Arbeit hatte Österreich naturgemäß in jenen Unterorganen des VN-SR, in denen ihm der Vorsitz übertragen worden war.

Den Vorsitz im 1267-Komitee, welches für die Führung der Al-Qaida/Taliban-Sanktionenliste zuständig ist, hatte Österreich gerade deshalb bewusst angestrebt, weil dessen Verfahren aus Sicht zahlreicher Kritiker - und auch nach Auffassung des Europäischen Gerichtshofs in Luxemburg - unter dem Gesichtspunkt der Rechtsstaatlichkeit und der Verfahrensgerechtigkeit reformbedürftig sind. Als Vorsitz des Komitees trug Österreich die Hauptverantwortung für die erste umfassende Sichtung der Liste in der Geschichte des Komitees. Insgesamt wurden in eineinhalbjähriger Arbeit 488 Einträge überprüft, davon rund 270 zum ersten Mal seit 2001; 46 Namen konnten von der Liste gestrichen werden; rund 50 weitere Delistinganträge sind noch in Bearbeitung. Im Dezember 2009 beteiligte sich Österreich aktiv an der Erarbeitung der SR-Resolution 1904 (2009), mit der das 1267-Sanktionenregime auch nach Meinung kritischer Beobachter substantiell verbessert werden konnte. Über österreichische Anregung wurde die Funktion einer unabhängigen Ombudsperson zur Prüfung von Anträgen auf Streichung von der Liste geschaffen. Als 1267-Vorsitz nahm Österreich aber darüber hinaus eine - va. für die Bemühungen der afghanischen Regierung zur Aussöhnung mit ehemaligen Taliban wichtige - politische Rolle wahr. Nach langwierigen Verhandlungen konnten elf frühere Taliban von der Liste gestrichen werden.

Als Vorsitz der Informellen Arbeitsgruppe für Internationale Tribunale konnte Österreich zum Kampf gegen die Straflosigkeit beitragen. In dieser Eigenschaft brachte Österreich insgesamt neun SR-Resolutionen zur Unterstützung der Arbeit der VN-Straftribunale für Jugoslawien (ICTY) und Ruanda (ICTR) ein. Nach fast zweijährigen Verhandlungen in der Arbeitsgruppe - den offenbar längsten Resolutionsverhandlungen in der Geschichte des VN-SR - nahm der VN-SR im Dezember 2010 Resolution 1966 (2010) an, mit der ab Mitte 2012 für das ICTR und ab Mitte 2013 für das ICTY ein „Restmechanismus“ geschaffen wird. Dieser soll v.a. gewährleisten, dass noch flüchtige Haupttäter wie Ratko Mladic oder Félicien Kabuga über das Ende der Tätigkeit dieser Ad-hoc Tribunale hinaus der internationalen Gerichtsbarkeit unterworfen bleiben.

Wenngleich Österreich dem VN-SR, wie eingangs erwähnt, im Berichtszeitraum zum dritten Mal angehörte, war dies die erste österreichische SR-Mitgliedschaft seit dem Beitritt zur Europäischen Union (EU) im Jahr 1995. Osterreich konnte im VN-SR so auch erstmals von seinen Erfahrungen als Teilnehmer an der Gemeinsamen Außen- und Sicherheitspolitik (GASP) der EU profitieren, deren Grundpositionen naturgemäß auch für die Festlegung der österreichischen Position im VN-SR von wesentlicher Bedeutung waren.

Zugleich hat sich Österreich (als das in diesen beiden Jahren neben Großbritannien und Frankreich einzige EU-Mitglied im VN-SR) in spezifischer Weise 
bemüht, den jeweiligen EU-Vorsitz, die übrigen EU-Mitglieder und die New Yorker EU-Delegation über alle relevanten Entwicklungen im VN-SR informiert zu halten. Über österreichische Initiative konnte die Hohe Vertreterin der Europäischen Union für Außen- und Sicherheitspolitik (EU-HV), Catherine Ashton, im Mai 2010 im Rahmen einer Debatte über die Zusammenarbeit zwischen VN und EU, an der auch Bundesminister Michael Spindelegger teilnahm, zum ersten Mal vor dem VN-SR sprechen.

Darüber hinaus sah sich Österreich (wie schon während seiner beiden vorangegangenen SR-Mitgliedschaften) gegenüber der Gesamtheit der VN-Mitglieder, von denen es ja mit großer Mehrheit in den VN-SR gewählt worden war, zur Transparenz über dessen Entscheidungen verpflichtet. Die Ständige Vertretung Österreichs bei den VN in New York (ÖV New York) hat sich deshalb sowohl gegenüber den anderen New Yorker Vertretungen als auch gegenüber der Zivilgesellschaft und den Vertretern der Medien um eine aktive Informationspolitik bemüht.

Österreich war überdies bestrebt, die SR-Mitgliedschaft zu nützen, um seine Stellung als Sitzstaat der VN und als Ort der internationalen Begegnung weiter zu stärken.

Im unmittelbaren Zusammenhang mit wesentlichen Themen der österreichischen SR-Arbeit standen die Westsahara-Gespräche in Dürnstein im August 2009, ein (in Zusammenarbeit mit der Universität Princeton organisiertes) „Afghanistan Implementation Colloquium“ Ende Februar 2010 in Baden und das informelle Treffen führender Vertreter Khartums und des Südsudan in Wien und Baden im November 2010. Im Dezember 2010 organisierte Österreich in Zusammenarbeit mit dem 1540-Komitee des VN-SR, das für Maßnahmen zur Bekämpfung einer möglichen Verbreitung von Massenvernichtungswaffen in den Händen von Terroristen zuständig ist, eine Konferenz in Wien, an der 25 Regionalorganisationen teilnahmen.

Mit starker internationaler Präsenz waren auch die - auf konkrete Arbeitsziele Österreichs im VN-SR ausgerichteten - „Alpbach-Retreats“ von 2009 und 2010 verbunden. Der Retreat von 2010 konnte überdies mit dem „Senior Management Retreat" des VN-GS verknüpft werden. VN-GS Ban Ki-moon, der in den Jahren 2009 und 2010 insgesamt dreimal nach Österreich kam, brachte aus diesem Anlass die gesamte Führungsspitze des VN-Sekretariats (die stellvertretende Generalsekretärin Asha-Rose Migiro, alle Untergeneralsekretäre und alle beigeordneten Generalsekretäre, insgesamt mehr als 70 Personen) nach Alpbach. Bereits im April 2010 befand sich VN-GS Ban Kimoon zu einem bilateralen Besuch in Österreich und nahm an einem Treffen des Koordinierungsrates der Leiter der Organisationen des VN-Systems (CEB) teil, welches auf Einladung des Generaldirektors der VN-Organisation für industrielle Entwicklung (UNIDO), Kandeh K. Yumkella, in Wien abgehalten wurde. 


\section{Einleitung}

In Österreich sind außerdem im Laufe der letzten zwei Jahre u.a. die folgenden neuen internationalen Einrichtungen mit VN-Konnex geschaffen worden:

- die Internationale Anti-Korruptionsakademie (IACA) in Laxenburg,

- ein Wiener Liaisonbüro des VN-Büros für Abrüstungsfragen (UNODA),

- das Wiener Zentrum für Abrüstung und Non-Proliferation,

- und ein Büro des International Peace Institute (IPI) in Wien.

Wenngleich es sich hier um Initiativen handelt, die in keinem ursächlichen Zusammenhang mit der österreichischen SR-Mitgliedschaft stehen, ist davon auszugehen, dass die erhöhte Sichtbarkeit Österreichs als SR-Mitglied im VN-Rahmen für die bereits seit längerer Zeit laufenden Bemühungen des BMeiA und damit für die Entscheidung, diese Einrichtungen in Wien anzusiedeln, förderlich war.

Zusammenfassend lässt sich daher aus der österreichischen SR-Mitgliedschaft folgendes ableiten: Nicht-ständige Mitglieder können durchaus eine gestaltende Rolle im VN-SR einnehmen. Wesentlich dafür ist aber eine klare inhaltliche Schwerpunktsetzung, wie dies Österreich auf Basis seiner bisherigen VN-Arbeit von Anbeginn angestrebt hat. Durch die Fokussierung auf Themen wie die Menschenrechte, die Rechtsstaatlichkeit, den Schutz von Zivilisten, insbesondere von Frauen und Kindern, sowie Fragen der NonProliferation von Massenvernichtungswaffen konnte Österreich zu konkreten Fortschritten in der Arbeit des VN-SR beitragen. Hiezu war es freilich auch nötig, die aktive Zusammenarbeit mit anderen SR-Mitgliedern zu suchen, die gleichartige Schwerpunkte vertreten, wie dies in den zwei Jahren unserer Mitgliedschaft insbesondere bei Mexiko und im ersten Jahr bei Costa Rica der Fall war.

Ein wichtiger Baustein einer erfolgreichen SR-Mitgliedschaft ist allerdings nicht nur die Verfolgung ausgewählter Schwerpunktinteressen, sondern die konstruktive und beharrliche Mitarbeit an der gesamten SR-Agenda unter Einschluss der sehr wichtigen Aktivitäten in den Sanktionenkomitees und anderen Unterorganen des VN-SR. Österreich hat sich gezielt um ein solches breites Engagement bemüht, was von seinen Partnern im VN-SR und vielen anderen VN-Mitgliedern, wie etliche Kommentare am Ende der SR-Mitgliedschaft gezeigt haben, offenbar geschätzt worden ist. Dies kann Österreich zweifelsohne auch bei seinen künftigen außenpolitischen Aktivitäten zugute kommen. Die VN werden in jedem Fall weiterhin einen zentralen Platz in der österreichischen Außenpolitik einnehmen. Das nächste wichtige Ziel ist für Österreich die Mitgliedschaft im VN-Menschenrechtsrat in der Periode 2011-2014.

Eine detailliertere Darstellung der Positionen, die Österreich zu den einzelnen Themen eingenommen hat, die 2009 und 2010 auf der Tagesordnung des VN-SR standen, findet sich in den anschließenden Abschnitten zu den einzelnen Tätigkeitsbereichen des VN-SR. 


\section{Die Tätigkeit des Sicherheitsrats und die österreichische Mitwirkung - Geografische Themen}

\section{a) Afrika}

Die Konflikte auf dem afrikanischen Kontinent bestimmten einen Großteil der Arbeit des VN-SR. Im Zentrum standen die Beiträge der VN zur Erhaltung des Friedens und zur Friedenskonsolidierung in der Region der Großen Seen, in Westafrika, im Sudan und am Horn von Afrika. Der VN-SR beschäftigte sich erstmals in umfassender Weise mit dem Problem der Piraterie vor der Küste von Somalia. Die vergangenen zwei Jahre waren durch eine wachsende Zusammenarbeit zwischen den VN, der AU und afrikanischen Regionalorganisationen - wie der ECOWAS - gekennzeichnet, zuletzt z.B. in Côte d'Ivoire. Die jährlichen Afrikareisen führten den VN-SR im Mai 2009 an den Sitz der AU in Addis Abeba, nach Ruanda, in die Demokratische Republik Kongo und nach Liberia. Im Oktober 2010 ging die Reise in den Sudan und nach Uganda. Österreich, das über ein historisch unbelastetes Verhältnis zu Afrika verfügt, hat sich in seiner Arbeit im VN-SR auf seinen Themenschwerpunkt, den Schutz der Zivilbevölkerung, insbesondere der Frauen und Kinder, konzentriert. Darüber hinaus ergaben sich auch etliche Möglichkeiten, die Arbeit der Wiener VN-Einrichtungen, insbesondere den Einsatz von UNODC im Kampf gegen den Drogenhandel in Westafrika, zu thematisieren. Der Beitrag und die Verantwortung betroffener Staaten wurden von Österreich in allen afrikanischen Angelegenheiten unterstrichen, was von den afrikanischen Mitgliedern im VN-SR geschätzt wurde.

\section{Sudan}

Der Sudan nahm in beiden Jahren eine zentrale Stellung in den Beratungen des VN-SR ein, sowohl im Hinblick auf die Situation in Darfur, als auch im Hinblick auf die Umsetzung des umfassenden Friedensabkommens (CPA) zwischen dem Nord- und dem Südsudan. Im Süden des Landes ist die VNMission im Sudan (UNMIS) stationiert. In Darfur befindet sich die gemeinsame Mission der VN und der AU (UNAMID) im Einsatz. Die humanitäre Situation und die Sicherheitslage blieben in beiden Einsatzgebieten über die gesamten zwei Jahre schwierig. In den letzten Monaten rückten die Vorbereitungen für das Unabhängigkeitsreferendum am 9. Jänner 2011 ins Zentrum der Aufmerksamkeit.

Die Reise des VN-SR in den Sudan im Oktober 2010 ermöglichte ein Treffen mit den Parteien des CPA im Süden (Juba) und im Norden (Khartum), um mit diesen über die Vorbereitungen zum Referendum über die Unabhängigkeit des Südsudan zu beraten und der Erwartungshaltung Ausdruck zu verleihen, dass das Referendum zeitgerecht abzuhalten und das Ergebnis zu res- 
pektieren ist. Der VN-SR hat auch die beiden VN-Missionen, UNMIS und UNAMID, besucht und ist mit VertreterInnen internationaler Hilfsorganisationen und der Zivilgesellschaft zusammen getroffen. In El Fasher (Darfur) wurde der VN-SR über die militärische und zivile Umsetzung des Auftrags zum Schutz der Zivilbevölkerung und zur Gewährleistung humanitärer Hilfe im Rahmen des Mandats von UNAMID informiert.

Die zentrale Bedeutung der Entwicklungen im Sudan kam auch in hochrangigen Treffen in New York zum Ausdruck. Am 24. September 2010 fand unter dem Vorsitz von VN-GS Ban Ki-moon ein hochrangiges Treffen zum Sudan statt, an dem neben den 15 Mitgliedern des VN-SR weitere 26 Staaten und Organisationen teilnahmen, darunter hochrangige Vertreter wie die sudanesischen Vizepräsidenten Ali Osman Taha („National Congress Party“, NCP) und Salva Kiir (,Sudan People‘s Liberation Movement“, SPLM), US-Präsident Obama und Bundesminister Michael Spindelegger. Im Vordergrund der Diskussionen standen die Vorbereitungen auf die Referenda (im Südsudan sowie in Abyei) und die Umsetzung des CPA. Am 16. November 2010 nahm Bundesminister Michael Spindelegger gemeinsam mit den AußenministerInnen der Vereinigten Staaten von Amerika, Nigerias sowie Bosnien und Herzegowinas an einer hochrangigen SR-Debatte zum Sudan unter dem Vorsitz des britischen Außenministers William Hague teil. Der VN-SR einigte sich auf eine Vorsitzerklärung (S/PRST/2010/24), in der die Parteien zur Implementierung des CPA und zu Fortschritten bei den Vorbereitungen für die Referenda aufgerufen wurden. Der VN-SR forderte auch Fortschritte im Friedensprozess betreffend Darfur (Doha-Prozess). Bundesminister Michael Spindelegger informierte über das österreichische Angebot, technische Expertise in der Frage der Staatsbürgerschaft zur Verfügung zu stellen.

Das Mandat von UNMIS wurde in beiden Jahren verlängert, zuletzt mit SRResolution 1919 (2010) bis 30. April 2011. Im Zentrum des Mandats steht die Unterstützung bei der Umsetzung des CPA. UNMIS stellte technische und logistische Unterstützung für die Vorbereitung der ersten Wahlen (10.-15. April 2010) seit 24 Jahren zur Verfügung. UNMIS unterstützte auch die Vorbereitung des Unabhängigkeitsreferendums vom 9. Jänner 2011. Österreich setzte sich v.a. für eine Priorisierung des Schutzes von Zivilisten im UNMISMandat sowie für eine vollständige Umsetzung desselben ein. UNMIS erstellte daher eine entsprechende Strategie, um während und nach dem Referendum auf Menschenrechtsverletzungen reagieren zu können. In Vorbereitung auf das Referendum wurde der VN-SR regelmäßig über die politischen Entwicklungen sowie die Sicherheitslage und die humanitäre Situation im gesamten Staatsgebiet des Sudan informiert. VN-GS Ban Ki-moon setzte zur Beobachtung des Referendums ein „Panel of Eminent Persons“ unter Leitung des ehemaligen Präsidenten von Tansania, Benjamin Mkapa, ein. In den Verhandlungen über die Zeit nach dem Referendum ging es u.a. um Fragen der Grenzziehung, Staatsbürgerschaft, Aufteilung der Öleinnahmen und der Bewegungsfreiheit von Nomaden. 
Betreffend die Situation in Darfur konnte eine Verbesserung der Beziehungen zwischen dem Tschad und Sudan 2010 auch zu einer gewissen Beruhigung der Situation im Grenzgebiet von Darfur beitragen. Am 23. Februar 2010 einigten sich die sudanesische Regierung und die Rebellengruppe JEM (,Justice and Equality Movement") in Doha, Katar, auf ein Rahmenabkommen zur Lösung des Darfur-Konflikts. Die Rebellengruppen LJM (,Liberation and Justice Movement") und SLA-Abdul Wahid (,Sudan Liberation Army“) traten trotz wiederholter Aufrufe durch den VN-SR im Laufe des Jahres dem DohaProzess nicht bei, es wurden aber Gespräche über ihren Beitritt zum Abkommen geführt. Trotz aller Bemühungen kommt es in Darfur weiterhin zu regelmäßigen gewalttätigen Auseinandersetzungen zwischen Regierungstruppen und Rebellen sowie zu Angriffen auf Blauhelme und humanitäres Personal. Das Mandat von UNAMID wurde in beiden Jahren verlängert, zuletzt mit SRResolution 1935 (2010) bis 31. Juli 2011. Die Resolution nimmt über Bemühen Österreichs eine Priorisierung des Mandats in Bezug auf den Schutz der Zivilbevölkerung und den Zugang humanitärer Organisationen vor. Auf Anregung Österreichs konnte sowohl in SR-Resolution 1881 (2009) als auch in SR-Resolution 1935 (2010) die explizite Forderung nach der Einhaltung der Menschenrechte und des humanitären Völkerrechts aufgenommen werden. Beide Missionen waren im Berichtszeitraum mit Einschränkungen in ihrer Bewegungsfreiheit konfrontiert, die ihnen die Umsetzung ihrer Mandate erschwerte. UNAMID erreichte überdies erst Ende 2010 rund $80 \%$ der autorisierten Stärke von rund 26.000 Militär- und Polizeikräften (vgl. 60 \% Ende 2008).

Der Chefankläger des Internationalen Strafgerichtshofs (IStGH) Luis Moreno Ocampo berichtete dem VN-SR halbjährlich über die in Verfolg zu SR-Resolution 1593 (2005) anhängigen Verfahren zur Situation in Darfur. Nachdem am 4. März 2009 die Vorverfahrenskammer des IStGH den Antrag auf Ausstellung eines Haftbefehls gegen den sudanesischen Präsidenten Omar Al-Bashir wegen Verbrechen gegen die Menschlichkeit und Kriegsverbrechen bestätigte, bemühten sich die afrikanischen Mitglieder im VN-SR - auf Basis von Entscheidungen der AU und der Arabischen Liga (AL) - um eine Suspendierung des Haftbefehls gegen Präsident Al-Bashir gemäß Art.16 Statut des IStGH. Nach dieser Bestimmung kann der VN-SR aus politischen Erwägungen im Einzelfall jeweils einen Aufschub einer Ermittlung oder einer Strafverfolgung für einen Zeitraum von zwölf Monaten verfügen. Für einen derartigen Beschluss konnte die erforderliche Mehrheit im VN-SR aber nicht gefunden werden. Der Sudan wies am Tag nach der Ausstellung des Haftbefehls 13 internationale, humanitäre Nichtregierungsorganisationen (NRO) an, ihre Tätigkeit in Darfur einzustellen, wodurch es über geraume Zeit zu einer weiteren Verschlechterung der humanitären Lage kam. Am 12. Juli 2010 folgte ein zweiter Haftbefehl gegen Präsident Al-Bashir wegen Völkermordes in drei Fällen.

Das von Botschafter Thomas Mayr-Harting geleitete 1591-Komitee ist für die Überwachung des Sanktionenregimes in Darfur zuständig. Dieses umfasst ein 
Waffenembargo und gezielte Sanktionen (Reiseverbot und Einfrieren von Vermögen) gegen Einzelpersonen. Es erwies sich für das Komitee als schwierig, ein Waffenembargo zu überwachen, welches sich nur auf einen Teil des Territoriums eines Landes bezieht und über Ausnahmebestimmungen verfügt, deren genauer Umfang innerhalb des Komitees umstritten war. Ein wesentliches Ergebnis der Tätigkeit des Komitees im Jahr 2009 war die Ausarbeitung allgemeiner Richtlinien über die Zusammenarbeit zwischen den vom VN-SR eingesetzten Expertengruppen und den jeweils relevanten FEO. Diese Richtlinien werden mittlerweile von allen Sanktionenkomitees des VN-SR angewandt, die mit FEO zusammenarbeiten. Im Jahr 2010 konzentrierte sich die Arbeit des Komitees u.a. darauf, Unternehmen für eine verstärkte Sorgfaltspflicht in Darfur zu sensibilisieren. SR-Resolution 1945 (2010) verlängerte das Mandat der Expertengruppe des Komitees um ein weiteres Jahr bis 19. Oktober 2011 und definierte die Ausnahmen vom Waffenembargo klarer als bisher, um in Zukunft eine bessere Überprüfung des Sanktionenregimes sicherzustellen.

\section{Somalia}

Die humanitäre Situation und die Sicherheitslage blieben schwierig, ruhige Phasen wurden immer wieder von neuerlichen Kampfhandlungen abgelöst. Versöhnung, Sicherheit und die Bereitstellung grundlegender Dienste für die Bevölkerung und die Einigung auf eine Verfassung, die auf breiter Basis beruht, bleiben die entscheidenden Aufgaben der Übergangsregierung vor Ende der Übergangszeit im August 2011. Das Mandat der seit 2007 aktiven Mission der AU in Somalia (AMISOM), sowie deren Autorisierung durch den VN-SR, wurden innerhalb der vergangenen zwei Jahre mehrmals verlängert. Die autorisierte Truppenstärke von 8.000 Personen wurde erst Ende 2010 erreicht (vgl. 4.000 Personen Ende 2008). Im Dezember 2010 wurde neben der Verlängerung der Autorisierung von AMISOM bis 30. September 2011 auch die autorisierte Stärke auf 12.000 Truppen erhöht. SR-Resolution 1863 (2009) autorisierte erstmals ein VN-Unterstützungspaket für die AUTruppen in Somalia. Dieses wurde seither mehrmals verlängert und zuletzt auch für die höhere Truppenzahl erweitert. In den Verhandlungen setzte sich Österreich u.a. erfolgreich für eine verstärkte Verantwortung aller Parteien für den Schutz von Zivilisten in SR-Resolution 1863 (2009) sowie für ein Ende der Rekrutierung von Kindersoldaten in SR-Resolution 1964 (2010) ein.

Der VN-SR autorisierte mit den SR-Resolutionen 1897 (2009) und 1950 (2010) Maßnahmen gegen die Piraterie vor der Küste Somalias für jeweils ein weiteres Jahr. Neuerungen im Vergleich zu den Vorjahren waren u.a. der Aufruf an alle VN-Mitglieder, Piraterie unter Strafe zu stellen und die Voraussetzungen für eine entsprechende Strafverfolgung zu schaffen. Mit SR-Resolution 1918 (2010) wurde der VN-GS erstmals aufgefordert, einen Bericht über mögliche Optionen zur Strafverfolgung von Piraten vorzulegen. In den Verhandlungen 
konnte auf österreichischen Vorschlag hin ein expliziter Verweis verankert werden, dass die Strafverfolgung und Inhaftierung von Piraten im Einklang mit den Menschenrechten zu erfolgen hat. Österreich setzte sich durchwegs für eine Diskussion im Gesamtkontext Somalias ein und verwies mehrmals auf die Wechselwirkung zwischen der schlechten Sicherheitslage und der Piraterie in Somalia sowie auf das umfassende EU-Engagement. Während des österreichischen SR-Vorsitzes wurde eine öffentliche Debatte zur Piraterie vor Somalia organisiert, an der neben den Mitgliedern auch direkt von der Piraterie Betroffene sowie an Anti-Piraterie Maßnahmen beteiligte Staaten und Organisationen teilnahmen. Die schwedische EU-Präsidentschaft informierte in dieser Debatte über die EUNAVFOR Somalia (Operation „Atalanta“).

Das bereits 1992 eingerichtete 751-Komitee überwacht das Sanktionenregime zu Somalia, welches ein Waffenembargo, Reiseverbote und Vermögenseinfrierungen umfasst. Die mit SR-Resolution 1907 (2009) verhängten Sanktionen gegen Eritrea (wegen dessen Unterstützung für bewaffnete Aufständische in Somalia) führte zu einer entsprechenden Erweiterung des bestehenden Komitees zu Somalia. Neben der Verlängerung der Monitoring-Gruppe des Komitees, brachte SR-Resolution 1916 (2010) weitere Neuerungen: Eine vorübergehende Ausnahme vom Finanzembargo wurde eingeführt für Zahlungen durch die VN, Spezialorganisationen und -programme und humanitäre Organisationen mit Beobachterstatus in der VN-GV, die notwendig sind, um die rechtzeitige Lieferung dringend benötigter humanitärer Hilfe in Somalia sicherzustellen. Außerdem stellt der VN-SR explizit klar, dass auch internationale, regionale (wie z.B. die EU) und subregionale Organisationen Ausnahmen vom Waffenembargo für Lieferungen und technische Unterstützung für Somalia beantragen können.

\section{Demokratische Republik Kongo}

Die Situation in der Demokratischen Republik Kongo bildete einen Schwerpunkt im VN-SR. Der Osten des Landes steht nach wie vor großen Herausforderungen gegenüber, dazu gehört u.a. mangelnde Staatsgewalt und die Bedrohung durch illegale bewaffnete Gruppen (v.a. „Lord’s Resistance Army“ - LRA, „Congrès national pour la défense du peuple“ - CNDP“, „Forces démocratiques de la libération du Rwanda“ - FDLR). Aber auch die mit dem illegalen Abbau von Bodenschätzen einhergehenden kriminellen Aktivitäten stellen weiterhin eine Herausforderung für eine nachhaltige Stabilisierung des Landes dar. Daher lag der Schwerpunkt der Reise des VN-SR in die Demokratische Republik Kongo im Mai 2009 auf dem Osten des Landes.

Der VN-Mission in der Demokratischen Republik Kongo (MONUC) kommt als primäre Aufgabe der Schutz der Zivilbevölkerung zu. Gleichzeitig hat der VN-SR MONUC das Mandat erteilt, die kongolesische Armee (FARDC) bei ihrem Kampf gegen die Rebellengruppe FDLR zu unterstützen. Im Zuge der gemeinsamen Militäroperationen Kimia II (2009) und Amani Leo (2010) kam 
es so allerdings zu einer Zusammenarbeit zwischen der VN-Mission und Einheiten der FARDC, denen gravierende Menschenrechtsverletzungen zur Last gelegt werden, was die VN starker Kritik aussetzte - auch seitens zahlreicher NRO. Anlässlich der Verlängerung des Mandats der Mission im Dezember 2009 gelang es Österreich und Costa Rica einigen Widerständen zum Trotz, in der SR-Resolution 1906 (2009) das Prinzip der „,conditionality policy“ zu verankern, die den VN die Verpflichtung auferlegt, den Streitkräften der Demokratischen Republik Kongo die Unterstützung zu entziehen, wenn die Gefahr schwerer Menschenrechtsverletzungen besteht.

Als mit Mai 2010 das Ende der durch SR-Resolution 1906 (2009) mandatierten Mission näher rückte, forderte die Regierung der Demokratischen Republik Kongo die Beendigung von MONUC, obwohl der Schutz der Zivilbevölkerung, die Hauptaufgabe der Mission, noch in keiner Weise gewährleistet erschien. Nach schwierigen Verhandlungen und einer weiteren Reise des VN-SR in die Demokratische Republik Kongo, konnte man sich allerdings darauf einigen, die Mission lediglich um ein (außerhalb der Konfliktzone im Osten eingesetztes) Bataillon zu verkleinern und bis 30. Juni $2010 \mathrm{zu}$ verlängern. Mit SR-Resolution 1925 (2010) wurde MONUC mit 1. Juli 2010 durch die VN-Stabilisierungsmission in der Demokratischen Republik Kongo (MONUSCO) abgelöst. Im Laufe dieser Diskussion verstärkte sich im VN-SR die Auffassung, dass Länder, in denen VN-Missionen mit Schutzmandaten zum Einsatz kommen, mit den VN eigentlich eine Art Vereinbarung (,compact") zur Bewältigung einer bestimmten Aufgabe eingehen, und es daher dem Geist dieser Abmachung widerspräche, die Zustimmung zum Verbleib der jeweiligen VN-Mission einseitig zurückzuziehen, bevor die gesetzte Aufgabe erfüllt ist.

Im Sommer $2010 \mathrm{kam}$ es zu Massenplünderungen und -vergewaltigungen durch Rebellengruppen in Walikale im Osten der Demokratischen Republik Kongo. MONUSCO kam diesbezüglich erneut unter starke Kritik, da die in der Nähe stationierten VN-Truppen nicht zum Schutz der Zivilbevölkerung eingegriffen und relevante Informationen erst mit geraumer Verspätung an den VN-SR übermittelt hätten. In den folgenden Konsultationen setzte sich Österreich u.a. für eine genaue Untersuchung der Vorfälle ein.

Das seit 2003 bestehende Sanktionenregime betreffend die Demokratische Republik Kongo sowie das Mandat der Expertengruppe des 1533-Komitees wurden regelmäßig verlängert. Mit SR-Resolution 1896 (2009) wurde außerdem das Mandat des 1533-Komitees um die Erstellung von Richtlinien betreffend Listing und Notifizierung von sanktionierten Personen erweitert. Mit SR-Resolution 1952 (2010) wurden nun erstmals Bestimmungen zur Sorgfaltspflicht festgeschrieben, durch die Risiken vermindert werden sollen, beim Handel mit Bodenschätzen aus dem Osten der Demokratischen Republik Kongo direkt oder indirekt illegale bewaffnete Gruppierungen, sanktionierte Personen, kriminelle Netzwerke und andere Gruppierungen, die schwere Menschenrechtsverletzungen begangen haben, zu unterstützen. 
Im Dezember 2010 einigte sich das Sanktionenkomitee auf die Verhängung gezielter Sanktionen (Reiseverbot und Einfrieren von Vermögen) gegen vier Einzelpersonen, die gegen das auferlegte Sanktionenregime verstoßen hatten.

\section{Tschad und Zentralafrikanische Republik}

Das Mandat der 2007 geschaffenen VN-Mission in der Zentralafrikanischen Republik und im Tschad (MINURCAT) konzentrierte sich von Beginn an auf den Schutz der Zivilbevölkerung in den Flüchtlingslagern im Osten des Tschad und im Nordosten der Zentralafrikanischen Republik. Die VN-Mission wurde nach dem Auslaufen der EU-Überbrückungsoperation EUFOR Tchad/RCA im März 2009 um eine militärische Komponente erweitert, an der sich auch Österreich bis Dezember 2009 beteiligte.

Anfang 2010 teilte der tschadische Präsident Idriss Déby den VN allerdings mit, dass sein Land einer Verlängerung von MINURCAT nicht mehr zustimmen wolle. Eine Reihe von SR-Mitgliedstaaten, darunter auch Österreich, das VN-Sekretariat und zahlreiche NRO zeigten sich über die Perspektive eines Abzugs von MINURCAT mehr als besorgt, da ein funktionierender Schutz der Zivilbevölkerung zu diesem Zeitpunkt alles andere als gewährleistet schien. In der Folge einigte sich der VN-SR nach zähen Verhandlungen mit SR-Resolution 1923 (2010) auf eine Verlängerung des MINURCATMandats bis Ende 2010, wobei die (verkleinerte) militärische Komponente der Mission nur mehr für den Schutz der zivilen VN-Präsenz verantwortlich war. Auf österreichisches Drängen wurde in SR-Resolution 1923 (2010) allerdings eine Notfallsklausel aufgenommen, die MINURCAT autorisierte, auf Akte der Gewalt gegen Zivilisten im unmittelbaren Umfeld der Mission zu reagieren. Außerdem schreibt die Resolution in sehr klarer Form die Verantwortung der tschadischen Regierung für den Schutz der betroffenen Zivilbevölkerung fest. Die seither feststellbaren Bemühungen des Tschad zum Aufbau der für diese Schutzaufgaben zuständigen Polizeikräfte sind zweifelsohne auch auf den im VN-SR erzeugten Druck zurückzuführen. In einer letzten Vorsitzerklärung (S/PRST/2010/29) zu MINURCAT vom 20. Dezember 2010 verlangte der VN-SR einen Bericht des VN-GS über die Entwicklung der Lage im Osten des Tschad im Hinblick auf den Schutz der Zivilbevölkerung, um die Nachverfolgung dieser Thematik durch den VN-SR sicherzustellen. Dieser Bericht soll sich insbesondere auf die Situation der Flüchtlinge und intern Vertriebenen, den humanitären Zugang sowie Maßnahmen zur Bekämpfung von Menschenrechtsverletzungen und die Sicherheitssituation im Allgemeinen konzentrieren.

Das Integrierte Büro der VN für die Friedenskonsolidierung in der Zentralafrikanischen Republik (BINUCA) löste 2009 die bisherige VN-Büro (BONUCA) ab. Die unterstützenden Aufgaben des VN-Büros konzentrieren sich u.a. auf den nationalen Dialog, Entwaffnung, Demobilisierung und Reintegration von Rebellen, Sicherheitssektorreform, Wiederherstellung der Staatsgewalt, Ach- 
tung der Menschenrechte und der Rechtsstaatlichkeit. Österreich hat sich zudem erfolgreich für die Ergänzung um ein Mandat zum Schutz von Kindern eingesetzt. BINUCA wurde zuletzt mit Vorsitzerklärung (S/PRST/ 2010/26) um zwölf Monate bis 31. Dezember 2011 verlängert. Während die Situation im Osten des Tschad von verbesserten Beziehungen mit dem Sudan profitieren konnte, ist das durch den Abzug von MINURCAT entstandene Sicherheitsvakuum im Nordosten der Zentralafrikanischen Republik sowie die von Rebellengruppen ausgehende Bedrohung besorgniserregend. Die Regierung der Zentralafrikanischen Republik ersuchte die VN nach dem Ende von MINURCAT um Unterstützung beim Kapazitätsaufbau der nationalen Armee.

\section{Côte d'Ivoire}

Im Zentrum der Arbeit des VN-SR zu Côte d'Ivoire stand während des Berichtszeitraums in erster Linie die Umsetzung des Ouagadougou-Abkommens. Die wichtigsten Punkte betreffen die Entwaffnung und Demobilisierung der Milizen, die Wiedervereinigung der seit dem Bürgerkrieg faktisch getrennten Landesteile, die Wiederherstellung der staatlichen Autorität im gesamten Staatsgebiet, die Durchführung von Wahlen und die nationale Aussöhnung. Der Leiter der VN-Mission in Côte d'Ivoire (UNOCI), SRSG Choi Young-jin, berichtete dem VN-SR regelmäßig über die Fortschritte in diesen Bereichen einschließlich der Unterstützung durch UNOCI. Das Mandat von UNOCI (seit 2004) wurde mehrmals verlängert, zuletzt mit SR-Resolution 1962 (2010) bis 30. Juni 2011. Die Truppenstärke von UNOCI wurde 2009 um ein Bataillon reduziert, eine weitere Reduktion sollte erst nach klaren Fortschritten in der Umsetzung des Friedensabkommens von Ouagadougou vom März 2007 stattfinden. Am 24. November 2010, unmittelbar vor dem zweiten Wahlgang der Präsidentschaftswahlen, autorisierte der VN-SR mit SR-Resolution 1951 (2010) den VN-GS, die VN-Mission UNOCI zunächst für die Dauer von vier Wochen mit VN-Truppen aus der im benachbarten Liberia stationierten VN-Mission (UNMIL) zu verstärken.

Nach dem erfolgreichen Abschluss der für die Präsidentschaftswahlen notwendigen Erstellung der Wählerlisten konnte die erste Runde der Präsidentschaftswahlen am 31. Oktober 2010 durchgeführt werden. Am 28. November 2010 fand der zweite Wahlgang zwischen Amtsinhaber Laurent Gbagbo und Alassane Ouattara statt. Das von der unabhängigen Wahlbehörde bekannt gegebene Wahlergebnis wies Ouattara mit über 54\% der Stimmen als Sieger der Wahlen aus. SRSG Choi bestätigte, in Ausübung seiner mit SR-Resolution 1765 (2007) übertragenen Zertifizierungsfunktion, am 3. Dezember 2010 nach genauer Prüfung des Wahlvorgangs das Ergebnis der Wahlen. Die Weigerung von Amtsinhaber Gbagbo, das Wahlergebnis anzuerkennen und das Amt an Ouattara zu übergeben, führte zu einer ernsten politischen Krise, bei der es in Folge auch zu gewalttätigen Auseinandersetzungen kam. Der VN-SR 
beriet mehrmals intensiv über die Entwicklungen in Côte d'Ivoire und stand in regelmäßigem Kontakt mit dem VN-Sekretariat und UNOCI. Nach erfolglosen Vermittlungsbemühungen von ECOWAS, sowie des ehemaligen südafrikanischen Präsidenten Thabo Mbeki (im Auftrag der AU), trafen die Staatsund Regierungschefs der ECOWAS zu einem außerordentlichen Gipfeltreffen zusammen und verabschiedeten ein Kommuniqué, in dem Ouattara als Wahlsieger anerkannt und Gbagbo zur Übergabe des Amtes aufgefordert wurde. Am 8. Dezember 2010 einigte sich der VN-SR auf eine Presseerklärung (SC/10105), die dazu aufrief, das Wahlergebnis zu respektieren und auf das ECOWAS-Kommuniqué verwies. Weiters erneuerte der VN-SR seine volle Unterstützung für UNOCI.

Das seit 2004 bestehende Sanktionenregime in Côte d'Ivoire und das Mandat der Expertengruppe des 1572-Komitees wurden in beiden Jahren verlängert, zuletzt mit SR-Resolution 1946 (2010) bis 30. April 2011.

\section{Guinea-Bissau}

Die Lage in Guinea-Bissau erwies sich in den Jahren 2009 und 2010 weiterhin als instabil. Im März 2009 waren der Präsident und der Generalstabschef ermordet worden, eine diesbezügliche Untersuchung konnte immer noch nicht vollständig abgeschlossen werden. Österreich plädierte wiederholt für ein Ende der Straflosigkeit in Guinea-Bissau und sprach sich nachdrücklich gegen willkürliche Inhaftierungen aus. Im April $2010 \mathrm{kam}$ es zu schweren militärischen Unruhen in Guinea-Bissau aufgrund mangelnder ziviler Kontrolle über die Streitkräfte sowie deren Verwicklung in den illegalen Drogenhandel. Die zivile Kontrolle über die Armee bleibt daher weiterhin zentrales Thema für eine nachhaltige Stabilisierung des Landes. Darüber hinaus stellen der Kampf gegen grenzüberschreitende organisierte Kriminalität und Korruption sowie eine umfassende Sicherheitssektorreform, inkl. der Einrichtung eines Pensionsfonds für Mitglieder der Armee, große Herausforderungen dar. Österreich setzte sich in ganz Westafrika, v.a. aber in Guinea-Bissau, für eine verstärkte Zusammenarbeit des VN-SR mit UNODC ein. UNODC-Exekutivdirektor Antonio Maria Costa informierte den VN-SR wiederholt über die Bedrohung, die Drogenhandel und organisierte Kriminalität in Westafrika darstellen.

Das am 1. Jänner 2010 geschaffene Integrierte VN-Büro für die Friedenskonsolidierung in Guinea-Bissau (UNIOGBIS) löste das bisherige VN-Büro (UNOGBIS) ab und soll die Regierung von Guinea-Bissau beim Kapazitätenaufbau, der Sicherheitssektorreform, der Bekämpfung des Drogenhandels, der Förderung der Menschenrechte und den Reformen im Bereich der guten Regierungsführung unterstützen sowie die Arbeit der VN-Kommission für Friedenskonsolidierung (PBC) in Guinea-Bissau fördern. Das Mandat wurde zuletzt mit SR-Resolution 1949 (2010) bis zum 31. Dezember 2011 verlängert. 


\section{Liberia}

In Liberia ist die Situation trotz Fortschritten in der Sicherheitssektorreform nicht nachhaltig stabilisiert, außerdem herrscht weiterhin Handlungsbedarf im Justiz- und Justizvollzugssystem sowie bei der Korruptionsbekämpfung. Weitere bedeutende Themen im Stabilisierungsprozess waren zuletzt die Bestätigung der Nominierungen für die nationale Menschenrechtskommission durch den liberianischen Senat sowie die Umsetzung der Empfehlungen der Wahrheits- und Versöhnungskommission. Daher verlängerte der VN-SR in beiden Jahren das Mandat der VN-Mission in Liberia (UNMIL), zuletzt mit SR-Resolution 1938 (2010) bis 30. September 2011. Bereits 2009 wurde das Mandat um die Unterstützung der liberianischen Regierung bei den Vorbereitungen für die Wahlen 2011 erweitert. Die 2008 beschlossene Truppenreduktion wurde 2009 abgeschlossen, eine weitere Truppenreduktion soll erst nach den Wahlen 2011 erfolgen.

Mit SR-Resolution 1903 (2009) hob der VN-SR das Waffenembargo gegen die liberianische Regierung auf. Die Bestimmungen zum Reiseverbot und zum Einfrieren von Vermögenswerten von Personen aus dem Umkreis des ehemaligen Präsidenten Charles Taylor, sowie das Mandat des Expertenpanels des 1521-Komitees zum Sanktionenregime für Liberia, wurden mehrmals verlängert, zuletzt mit SR-Resolution 1961 (2010) bis 16. Dezember 2011.

\section{Sierra Leone}

Trotz der Fortschritte in den vergangenen Jahren blieb die Situation in Sierra Leone fragil. Das Integrierte VN-Büro für Friedenskonsolidierung in Sierra Leone (UNIPSIL) gilt als ein Modellbeispiel für eine politische VN-Mission. Vor allem die weit verbreitete Jugendarbeitslosigkeit und der Kampf gegen den illegalen Drogenhandel und das organisierte Verbrechen stellen eine große Herausforderung für die nachhaltige Entwicklung Sierra Leones dar. Der Leiter von UNIPSIL, Michael von der Schulenburg, berichtete im März 2010 von Fortschritten in der Korruptionsbekämpfung, informierte den VN-SR im September 2010 jedoch auch über seine Sorge betreffend die intransparente Lizenzvergabe zum Abbau der in Sierra Leone in großen Mengen vorhandenen Bodenschätze. Das Mandat von UNIPSIL wurde mehrmals verlängert, zuletzt mit SR-Resolution 1941 (2010) bis 15. September 2011. Die Resolution betonte die Notwendigkeit der Umsetzung der Strategiedokumente für Sierra Leone, der „Joint Vision“ sowie der „Agenda for Change“, die von UNIPSIL gemeinsam mit dem VN-Länderteam und der Regierung sowie der PBC erreicht werden soll. Hauptmandat der Mission ist die Unterstützung bei der Vorbereitung für die Wahlen 2012, für Maßnahmen im Bereich der guten Regierungsführung und der Menschenrechte sowie im Kampf gegen Jugendarbeitslosigkeit, organisiertes Verbrechen, Drogenhandel und Korruption. 
Das letzte Verfahren vor dem Sondergerichtshof für Sierra Leone gegen den ehemaligen Präsidenten Liberias Charles Taylor wurde in Den Haag weitergeführt und war mit Ende 2010 noch nicht abgeschlossen.

Der VN-SR nahm am 29. September 2010 einstimmig die SR-Resolution 1940 (2010) an und löste dadurch mit sofortiger Wirkung das mit SR-Resolution 1132 (1997) eingerichtete Sanktionenregime auf.

Sierra Leone ist neben Burundi, Liberia, Guinea-Bissau und der Zentralafrikanischen Republik das fünfte Land auf der Agenda der PBC. Österreich wurde im Juli 2009 Mitglied der sog. länderspezifischen Konfiguration für Sierra Leone.

\section{Burundi}

Das 2006 eingerichtete Integrierte VN-Büro in Burundi (BINUB) wurde durch SR-Resolution 1959 (2010) mit 1. Jänner 2011 durch das neue VN-Büro in Burundi (BNUB) abgelöst und soll die Regierung u.a. in folgenden Bereichen unterstützen: Aufbau bzw. Stärkung von nationalen Institutionen, Förderung des interparteilichen Dialogs, Bekämpfung der Straflosigkeit und Fortschritte im Bereich Übergangsgerichtsbarkeit, Monitoring von Menschenrechten und diesbezüglichen Kapazitätsaufbau sowie Senkung der Armut. Besonders hervorzuheben sind die erfolgreiche Durchführung von fünf Wahlen und die Entwicklungen im Bereich der Übergangsgerichtsbarkeit 2010. Trotz der Fortschritte in der Friedenskonsolidierung in Burundi weisen Teile des letzten VN-GS Berichts noch auf Herausforderungen v.a. in den Bereichen Rechtsstaatlichkeit, politischer Dialog und Menschenrechte hin.

Auch in Burundi musste sich der VN-SR in den Jahren 2009/2010 mit der Frage der Zustimmung des Gastlandes für eine VN-Präsenz befassen: Im Dezember 2009 verlangte die Regierung den Abzug des damaligen Exekutivbeauftragten des VN-GS (ERSG), Youssef Mahmoud, da dieser zu einseitig mit den Oppositionsparteien kooperiert habe. SR-Resolution 1902 (2009) fordert den ERSG allerdings auf, mit allen Parteien einen Dialog zu führen. Österreich hat sich im Vorfeld der Wahlen 2010 dafür eingesetzt, dass der VN-SR einen intensiveren Dialog mit der länderspezifischen Konfiguration der PBC eingeht, wie es mit Liberia im Rahmen eines informellen Dialoges im November 2010 der Fall war.

\section{Westsahara}

Österreich hat den Anfang 2009 ernannten Persönlichen Gesandten des VN-GS für die Westsahara, Christopher Ross, von Beginn an in seinen Bemühungen, eine Wiederaufnahme der Friedensgespräche zwischen Marokko und der Polisario zu erreichen, unterstützt. So fanden auf Einladung Österreichs im August 2009 in Dürnstein informelle Gespräche der Parteien 
statt. Dieses erste Zusammentreffen von Marokko und der Polisario seit der Verhandlungsrunde in Manhasset im März 2008 sowie die erstmalige Erörterung von Menschenrechtsfragen stellten einen großen Erfolg dar. Ebenfalls in Dürnstein anwesend waren Vertreter Algeriens und Mauretaniens. Das respektvolle und offene Gesprächsklima und die Rolle Österreichs als ehrlicher Makler wurden von den Anwesenden ausdrücklich gewürdigt. Seither hat Österreich wiederholt seine Bereitschaft ausgedrückt, im Sinne seiner Rolle als Plattform für Frieden und Dialog, als Austragungsort für weitere Gespräche zur Belebung des politischen Prozesses zur Verfügung zu stehen.

Anlässlich der jährlichen Verlängerungen des Mandats der 1991 eingerichteten Mission der VN für das Referendum in der Westsahara (MINURSO) konnte Österreich u.a. folgende Akzente setzen: Es gelang Österreich in den kontroversiell geführten Verhandlungen, dass die Bedeutung von Fortschritten in der menschlichen Dimension des Konflikts für die Vertrauensbildung und den Dialog zwischen den Parteien erstmals ausdrücklich anerkannt wurde (SR-Resolution 1871 (2009)). Weiters setzte sich Österreich im VN-SR für die Empfehlung des VN-GS zum Dialog der Parteien mit dem Büro des VN-Hochkommissars für Menschenrechte (OHCHR) ein (SR-Resolution 1920 (2010)).

Der VN-SR beschäftigte sich ebenfalls mit der Räumung des Zeltlagers Gdim Izik bei El Aaiun durch marokkanische Sicherheitskräfte im November 2010. Österreich unterstützte die Abhaltung von Konsultationen des VN-SR.

\section{Regionale Themen}

\subsection{VN-Regionalbüro in Westafrika}

Das Mandat des VN-Regionalbüros in Westafrika (UNOWA) wurde im Dezember 2010 um weitere drei Jahre verlängert (S/2010/661). Diese 2001 eingerichtete relativ kleine politische Mission wurde geschaffen, um die Zusammenarbeit zwischen den VN und anderen Partnern in der Region zu verbessern. Im Zentrum der Arbeit stehen Konfliktprävention und -lösung in der Region, sowie themenübergreifende und grenzüberschreitende Entwicklungen und aktive Zusammenarbeit mit ECOWAS. Österreich stellte von September 2006 bis Juli 2009 mit Oberstleutnant Georg Moik den stellvertretenden Militärberater bei UNOWA. Der Leiter von UNOWA, SRSG Said Djinnit, berichtete dem VN-SR halbjährlich über die Arbeit des Büros und die Entwicklungen in der Region. Das neue Mandat von UNOWA legt einen stärkeren Fokus auf Sicherheitssektorreform, v.a. im Hinblick auf transnationale organisierte Kriminalität (Drogen- bzw. Menschenhandel, Handel mit Kleinwaffen und leichten Waffen - KLW) sowie auf Mainstreaming von GenderThemen (inkl. Umsetzung von SR-Resolution 1325 (2000)). 


\subsection{Ad-Hoc Arbeitsgruppe für Konfliktprävention und -lösung in Afrika}

Die 2002 gegründete Ad-Hoc Arbeitsgruppe für Konfliktprävention und Konfliktlösung in Afrika soll die Umsetzung von entsprechenden Empfehlungen überprüfen, Vorschläge zu einer verbesserten Kooperation zwischen VN-SR und Wirtschafts- und Sozialrat (ECOSOC) sowie anderen VN-Entitäten abgeben, regionale und konfliktübergreifende Themen überprüfen, welche die Arbeit des VN-SR zu Konfliktprävention und -lösung in Afrika betreffen sowie Empfehlungen zu einer verbesserten Zusammenarbeit des VN-SR mit afrikanischen Regional- und Subregionalorganisationen formulieren. Hauptthema der vergangenen zwei Jahre war v.a. eine verbesserte Kooperation des VN-SR mit dem Friedens- und Sicherheitsrat der AU. Diesbezüglich fand 2009 in Addis Abeba und 2010 in New York ein Zusammentreffen beider Räte statt, bei welchen sich die jeweiligen Mitgliedstaaten über diverse Konfliktsituationen sowie über die Modalitäten einer möglichen engeren Zusammenarbeit austauschen konnten.

\subsection{VN-Regionalbüro in Zentralafrika}

Im Laufe der österreichischen SR-Mitgliedschaft einigte sich der VN-SR auch auf die Einrichtung eines Regionalbüros der VN für Zentralafrika (UNOCA). Diesbezügliche Verhandlungen begannen bereits vor der österreichischen SR-Mitgliedschaft und konnten im August 2010 abgeschlossen werden. UNOCA, welches sich u.a. auch mit der regionalen Bedrohung durch die LRA befassen soll, nahm per 1. Jänner 2011 seine Tätigkeit in Libreville, Gabun, auf. Das Mandat des Büros läuft vorerst für zwei Jahre.

\subsection{Offene Debatte zum illegalen Klein- und Leichtwaffenhandel in Zentralafrika}

Am 19. März 2010 fand auf Initiative Gabuns eine offene SR-Debatte über die Auswirkungen des illegalen Handels mit KLW auf Zentralafrika statt, an der neben der stellvertretenden VN-GS Asha-Rose Migiro auch UNODCExekutivdirektor Costa und der Generalsekretär der zentralafrikanischen Wirtschaftsgemeinschaft (ECCAS) teilnahmen. Im Zentrum der Diskussion standen die zahlreichen negativen Auswirkungen des illegalen Handels mit KLW wie z.B. die Prolongierung bewaffneter Konflikte, Gefährdung friedenskonsolidierender Initiativen, Gewalt gegen Zivilisten, v.a. Frauen und Kinder, sowie Menschenrechtsverletzungen. Hervorgehoben wurde auch die Bedeutung (sub)regionaler und internationaler Kooperation sowie der Unterstützung der zentralafrikanischen Staaten beim Kapazitätsaufbau durch die internationale Gemeinschaft. Die nach der offenen Debatte angenommene Vorsitzerklärung (S/PRST/2010/6) enthält außerdem einen Aufruf zum Beitritt zur VN-Konvention gegen transnationales organisiertes Verbrechen 
(UNTOC) und die Unterstützung der ECCAS-Initiative für einen Waffenkontrollvertrag. Österreich setzte sich nicht nur im Zusammenhang mit Afrika, sondern auch im Rahmen der Sanktionenkomitees für eine starke Waffenexportkontrolle ein.

\section{Sonstige Konflikte}

Wenn Konfliktsituationen nicht auf der Agenda des VN-SR stehen, die Lage vor Ort jedoch eine Auseinandersetzung mit den betroffenen Ländern erfordert, werden diese oft unter den allgemeinen Titeln „Frieden und Sicherheit in Afrika“ oder „Friedenskonsolidierung in Westafrika“ behandelt.

\subsection{Eritrea und Dschibuti}

Als Reaktion auf die Kampfhandlungen an der Grenze zwischen Eritrea und Dschibuti am 10. Juni 2008 und einem entsprechenden VN-Untersuchungsbericht, rief der VN-SR mit SR-Resolution 1862 (2009) Eritrea auf, seine Soldaten aus dem Grenzgebiet mit Dschibuti abzuziehen und Wege zu suchen, diesen Grenzkonflikt friedlich zu lösen. Im Juni 2010 einigten sich die Konfliktparteien auf Katar als Mediator. Die Staatsoberhäupter unterzeichneten am 7. Juni 2010 ein entsprechendes Abkommen. Bis eine Lösung gefunden wird, überwachen katarische Truppen das umstrittene Grenzgebiet.

\subsection{Madagaskar}

Nach mehreren Wochen teils blutiger Auseinandersetzungen zwischen den Anhängern des damaligen Präsidenten Marc Ravalomanana und dem damaligen Bürgermeister Andry Rajoelina im Frühjahr 2009, gab Ravalomanana seinen Rücktritt bekannt und Rajoelina erklärte sich selbst zum Präsidenten. In Verfolg zu den Diskussionen um den Machtwechsel in Madagaskar wurde eine Vorsitzerklärung (S/PRST/2009/2) verhandelt, die das Wiederaufleben von verfassungswidrigen Regierungswechseln in Afrika verurteilt, ohne dabei jedoch spezifisch auf Madagaskar einzugehen.

\subsection{Guinea}

In Guinea kam es am 28. September 2009 zu gewaltsamen Übergriffen der Sicherheitskräfte auf Demonstranten, bei denen rund 150 Menschen ums Leben kamen. Eine VN-Untersuchungskommission kam zu dem Schluss, dass das Massaker geplant war und ein Verbrechen gegen die Menschlichkeit darstellt. Nach mehreren Konsultationen und Vorsitzerklärungen (S/PRST/2010/3, S/PRST/2009/27) des VN-SR sowie Vermittlung zwischen den Konfliktparteien im Jahr 2010 konnte SRSG Djinnit im November 2010 dem VN-SR schließlich über eine friedlich verlaufene zweite Runde der Präsidentenwahlen berichten. 


\section{b) Naher und Mittlerer Osten}

\section{Israel / Palästina}

Die Situation im Nahen Osten stellt nach wie vor einen der großen Brennpunkte internationaler Politik und somit auch einen Fokus der Arbeit des VN-SR dar. Dies spiegelte sich in den monatlichen Nahostdebatten des VN-SR wider.

Gleich zu Beginn der österreichischen SR-Mitgliedschaft fanden im VN-SR in Reaktion auf die am 27. Dezember 2008 ausgebrochenen Kampfhandlungen im und um den Gaza-Streifen intensive Beratungen statt. Österreich trug in den Verhandlungen, welche auch auf Ministerebene unter erstmaliger persönlicher Teilnahme von Bundesminister Michael Spindelegger geführt wurden, im VN-SR zu einer Verständigung zwischen den arabischen Staaten und den P-5 bei. In der Folge gelang es, trotz anfänglich großer Auffassungsunterschiede, eine Resolution (SR-Resolution 1860 (2009)) zu verabschieden. Diese erste unter österreichischer SR-Mitgliedschaft angenommene Resolution forderte u.a. eine sofortige, dauerhafte und umfassend eingehaltene Waffenruhe sowie die ungehinderte Versorgung und Verteilung humanitärer Hilfe in Gaza. Angesichts der dramatischen Versorgungslage der Zivilbevölkerung war Österreich die Forderung nach einer nachhaltigen Öffnung der Übergänge in den Gazastreifen ein besonderes Anliegen. Weiters setzte sich Österreich für die strikte Einhaltung des humanitären Völkerrechts ein und forderte von allen Parteien die Untersuchung von Vorwürfen von Verstößen gegen Menschenrechte und humanitäres Völkerrecht. Im Zusammenhang mit Angriffen auf VN-Einrichtungen trat Österreich für die rasche Umsetzung der Empfehlungen der VN-internen Untersuchungskommission ein. In Verfolg der Gaza Krise wurde vom VN-Menschenrechtsrat die VN-Ermittlungsmission für den Gaza-Konflikt (UNFFM) unter Führung von Richard Goldstone eingesetzt. Österreich hat gemeinsam mit seinen EU-Partnern das umfassende Mandat dieser Mission unterstützt.

Bundesminister Michael Spindelegger nahm weiters am 11. Mai 2009 an einer vom russischen Außenminister Sergej Lawrow geleiteten Ministerdebatte teil, in deren Rahmen eine Vorsitzerklärung (S/PRST/2009/14) angenommen wurde. Diese unterstrich die Dringlichkeit der Wiederaufnahme der israelisch-palästinensischen Friedensverhandlungen, bekräftigte das Bekenntnis des VN-SR zur Zwei-Staaten-Lösung und mahnte erneut die volle Umsetzung der „Road-Map Verpflichtungen“ durch beide Parteien ein.

Anlässlich der Erstürmung der Gaza-Hilfsflottille am 31. Mai 2010 durch Israel hat sich Österreich im VN-SR für eine rasche und umfassende Untersuchung des Vorfalls sowie erneut für die Öffnung aller Grenzübergänge für humanitäre Lieferungen in den Gaza-Streifen eingesetzt. Weiters unterstützte Österreich die Einrichtung eines Untersuchungspanels durch den VN-GS. Diese österreichischen Forderungen fanden sich zum Teil in der am 1. Juni 2010 verabschiedeten Vorsitzerklärung (S/PRST/2010/9) wieder. In dieser 
rief der VN-SR u.a. zu einer prompten, unparteiischen, glaubwürdigen und transparenten Untersuchung im Einklang mit internationalen Standards auf.

In weiterer Folge begrüßte der VN-SR in einer Presseerklärung (SC/10001) vom 3. August 2010 die Einrichtung einer Kommission zur Untersuchung des Zwischenfalls durch den VN-GS und das Bekenntnis Israels und der Türkei zur umfassenden Zusammenarbeit mit dem gemischt israelisch-türkischen Gremium unter Leitung des ehemaligen neuseeländischen Premierministers Geoffrey Palmer und des ehemaligen kolumbianischen Staatspräsidenten Alvaro Uribe.

Darüber hinaus unterstrich Österreich im VN-SR regelmäßig die Wichtigkeit der Arbeit sowie die Notwendigkeit der anhaltenden internationalen Unterstützung für das VN-Hilfswerk für Palästinaflüchtlinge im Nahen Osten (UNRWA). UNRWA musste in Ermangelung einer umfassenden Friedenslösung im Jahr 2009 den sechzigsten Jahrestag seiner Tätigkeit begehen und erbringt unverändert essentielle öffentliche Dienstleistungen (u.a. in den Bereichen Ausbildung und Gesundheit).

\section{Libanon}

Im Südlibanon kam die Brüchigkeit der Waffenruhe im Berichtszeitraum durch wiederholten Raketenbeschuss israelischen Territoriums, Gegenbeschuss durch die israelische Armee, regelmäßige Verletzungen des libanesischen Luftraums durch die israelische Luftwaffe sowie weitere Zwischenfälle immer wieder zum Ausdruck. Tragischer Höhepunkt dieser Fragilität waren am 3. August 2010 die schwersten Auseinandersetzungen zwischen israelischer und libanesischer Armee seit 2006 mit Toten und Verletzten auf beiden Seiten. Der VN-SR rief die Parteien zur äußersten Zurückhaltung und zur Einhaltung der Waffenruhe auf (Bemerkungen des SR-Vorsitzes gegenüber der Presse). Diese Vorfälle verdeutlichten einmal mehr, dass ein dauerhafter Frieden zwischen Israel und Libanon sowie eine umfassende Lösung des Nahostkonflikts für die Stabilität und nachhaltige Entwicklung der Region unerlässlich sind.

Insgesamt gelang es der Interimstruppe der VN im Libanon (UNIFIL), die gespannte Ruhe südlich des Litani-Flusses weitgehend aufrechtzuerhalten. Allerdings sah sich UNIFIL auch mit Einschränkungen seiner Bewegungsfreiheit konfrontiert, die Österreich wiederholt verurteilte. Weiters forderte Österreich generell die Gewährleistung der Sicherheit von VN-Personal ein. Nach Zusammenstößen mit Gruppen protestierender Zivilisten während und nach einem UNIFIL-Großmanöver Ende Juni 2009 verurteilte der VN-SR in einer Presseerklärung am 9. Juli 2009 (SC/9976) diese Zwischenfälle und forderte die Gewährleistung der Bewegungsfreiheit und Sicherheit der VN-Truppen ein.

Österreich setzte sich in den Beratungen des VN-SR zum Libanon für die Beendigung der Verletzungen des libanesischen Luftraums durch israelische 
Überflüge, den Rückzug der israelischen Truppen aus Ghajar und dem Gebiet der Shebaa Farmen im Südlibanon sowie die Entwaffnung von Hisbollah und palästinensischen Milizen in den UNRWA Flüchtlingslagern im Libanon ein. Angesichts anhaltender Berichte über Waffenschmuggel in den Südlibanon forderte Österreich ebenfalls die strikte Einhaltung des Waffenembargos. Das Mandat von UNIFIL wurde mit den SR-Resolutionen 1884 (2009) bzw. 1937 (2010) einstimmig jeweils um ein Jahr verlängert, zuletzt bis 31. August 2011.

Der VN-SR wurde auch zweimal vom VN-Sondergesandten für die Umsetzung der SR-Resolution 1559 (2004), Terje Rød Larsen, über relevante Entwicklungen informiert. Mit dieser Resolution wurde der Abzug aller ausländischen Truppen aus dem Libanon und die Entwaffnung und Auflösung aller im Libanon aktiven Milizen gefordert. Weiters wurde der libanesischen Regierung Unterstützung bei der Erweiterung ihrer Kontrolle auf das gesamte Staatsgebiet zugesagt.

\subsection{Sondertribunal}

In einer Presseerklärung am 3. März 2009 (SC/9606) begrüßte der VN-SR die zwei Tage zuvor erfolgte Aufnahme der Arbeiten des Sondertribunals für den Libanon in Den Haag als wichtigen Schritt, um die Verantwortlichen für die Ermordung des ehemaligen libanesischen Premierministers Rafik Hariri und anderer Opfer vom 14. Februar 2005 zur Verantwortung zu ziehen. Der Leiter der bis dahin tätigen Unabhängigen Internationalen Untersuchungskommission, Daniel Bellemare, setzte die Untersuchung des Bombenanschlags als Chefankläger des Sondertribunals fort. Das Jahr 2010 war geprägt von Spekulationen um mögliche Anklageerhebungen des Sondertribunals, die zunehmend innenpolitische Spannungen nährten. Am 5. November 2010 befasste sich der VN-SR mit Übergriffen auf ein Ermittlerteam des Büros des Chefanklägers des Sondertribunals in Beirut. Österreich setzte sich in diesem Zusammenhang für die Wahrung der Unabhängigkeit des Sondertribunals ein und unterstrich seine Unterstützung für dessen Tätigkeit im Dienste der Bekämpfung der Straflosigkeit und Stärkung der Rechtsstaatlichkeit.

\section{Syrien}

Ein wesentlicher Teil des aktiven österreichischen Engagements im Nahen Osten ist die Beteiligung an der Beobachtertruppe der VN für die Truppenentflechtung in den Golanhöhen (UNDOF). Österreich nimmt seit 1974 an UNDOF teil und entsendet derzeit mit bis zu 387 SoldatInnen das stärkste Truppenkontingent. Der Auslandseinsatz des Bundesheeres auf dem Golan ist somit der größte im Rahmen einer FEO der VN. Österreich verfügt aufgrund der 36-jährigen Beteiligung an UNDOF (und weiters durch Entsendung von Militärbeobachtern zur Organisation der VN zur Überwachung des 
Waffenstillstands - UNTSO) über große Erfahrung in der Region. Die hohe Wertschätzung der VN für das langjährige österreichische Engagement am Golan drückt sich auch in der wiederholten Ernennung von österreichischen Offizieren zu UNDOF-Truppenkommandanten aus, zuletzt Generalmajor Wolfgang Jilke von 2007-2010. Der VN-SR verlängerte das Mandat von UNDOF jeweils für weitere sechs Monate, zuletzt mit SR-Resolution 1965 (2010) bis zum 30. Juni 2011.

\section{Iran}

Der Iran konnte die Bedenken der internationalen Gemeinschaft über eine mögliche militärische Komponente seines Nuklearprogramms aufgrund der Nichtumsetzung der seit 2006 angenommenen Resolutionen des VN-SR und des IAEO-Gouverneursrats nicht ausräumen. Trotz der langjährigen Bemühungen der E3+3 zur Lösung des iranischen Nukleardossiers konnte der Iran das Vertrauen in die ausschließlich friedliche Natur seines Nuklearprogramms nicht herstellen. Daher nahm der VN-SR am 9. Juni 2010 nach intensiven Beratungen SR-Resolution 1929 (2010) mit zwölf Ja-Stimmen, zwei Nein-Stimmen (Brasilien, Türkei) und einer Enthaltung (Libanon) an. Die Resolution verfolgte das primäre Ziel, die Entwicklung des iranischen Nuklear- und Raketenprogramms zu verlangsamen bzw. zu stoppen. Daher wurden die bestehenden Sanktionsmaßnahmen verstärkt bzw. zielgerichtet erweitert (u.a. durch Ausweitung des Waffenembargos, durch Reiseverbote für und Vermögenseinfrierungen von zusätzlichen iranischen Personen/Entitäten, durch Verschärfung des Kargoinspektionsregimes einschließlich in internationalen Gewässern, des Verbots ballistischer Raketenstarts, durch die Vernichtung beschlagnahmter illegaler Lieferungen durch VN-Mitgliedstaaten, Beschränkungen im Finanz-, Handels- und Bankensektor sowie die Etablierung eines Expertenpanels). Österreich hat sich konstruktiv in die Resolutionsverhandlungen eingebracht und sich generell für eine Verstärkung der Rechtssicherheit und -klarheit des Resolutionstexts engagiert. Weiters wurde auf österreichisches Betreiben die Aufforderung zur raschen Ratifizierung des Vertrags über das umfassende Verbot von Nuklearversuchen (CTBT) und des Zusatzprotokolls zum IAEO-Sicherheitskontrollabkommen verankert.

In seiner Votumserklärung anlässlich der Annahme der SR-Resolution 1929 (2010) betonte Österreich seine Unterstützung der langjährigen EU Politik des doppelgleisigen Ansatzes (diplomatische Verhandlungen mit dem Iran parallel zu einer Verschärfung der Sanktionen bei Nichtkooperation) und forderte den Iran erneut auf, das Gesprächsangebot von EU-HV Ashton anzunehmen. In diesem Sinne unterstützte Österreich die beiden E3+3 Anreizpakete aus 2006 bzw. 2008 in der Hoffnung, dass der Iran ohne Vorbedingungen den Dialog mit den E3+3 und EU-HV Ashton aufnehmen werde, um eine umfassende Lösung des Nukleardossiers zu erzielen. Diese Position hatte Bundesminister Michael Spindelegger auch anlässlich des Besuchs des 
iranischen Außenministers Manouchehr Mottaki in Wien im April 2010 unmissverständlich zum Ausdruck gebracht.

Das mit SR-Resolution 1737 (2006) eingerichtete Sanktionenkomitee setzte seine Überwachungstätigkeiten fort. Mit SR-Resolution 1929 (2010) wurde vorerst für ein Jahr ein neues Expertenpanel eingerichtet. Dieses wurde dem 1737-Komitee in formeller Sitzung am 10. Dezember 2010 vorgestellt und soll das Komitee durch Informationsanalysen, Empfehlungen und Berichte unterstützen. Das Expertenpanel führte bereits am 14. Dezember 2010 in Wien Arbeitsgespräche mit VertreterInnen des BMeiA und anderen Ressorts. In diesem Rahmen wurden die Experten über die österreichischen Bemühungen zur Umsetzung seiner Verpflichtungen gemäß der einschlägigen SRResolutionen sowie über die EU-autonomen Sanktionen gegen den Iran umfassend informiert.

\section{Irak}

Der Leiter der Unterstützungsmission der VN für den Irak (UNAMI) und SRSG (Staffan de Mistura, bzw. seit Juli 2009 Ad Melkert) unterrichtete den VN-SR vierteljährlich über die Lage im Irak. Österreich unterstützte die Bemühungen von UNAMI um eine nationale Aussöhnung und die Stärkung von Menschenrechtsschutz und Rechtsstaatlichkeit sowie die aktive Einbindung der irakischen Frauen am Wiederaufbau des Landes. Österreich trat insbesondere für die Mitwirkung von Frauen bei der Neugestaltung des Sicherheitsapparates ein, der im Zuge des Rückzugs der US-Truppen zunehmend Verantwortung für die Gewährleistung der öffentlichen Sicherheit übernahm. Darüber hinaus führte Österreich den Vorsitz des Verwaltungsrats der VN-Entschädigungskommission (UNCC). Diese ist ein gemäß SR-Resolution 692 (1991) eingerichtetes, subsidiäres Organ des VN-SR zur Abwicklung von Schadenersatzansprüchen aus der irakischen Besetzung Kuwaits 1990/91 mit Sitz in Genf. Die österreichische Position spiegelte einerseits Unterstützung für die Bemühungen des Iraks, seinen internationalen Status vor 1991 vollständig wiederherzustellen, andererseits Anerkennung legitimer kuwaitischer Sicherheitsanliegen wider.

Die VN konzentrierten ihre Tätigkeit im Irak zunächst auf die Regionalwahlen 2009 und die Vorbereitung der nationalen Wahlen 2010 sowie auf Bemühungen zur Lösung von Binnengrenzkonflikten und offenen Verfassungsfragen. Nach der aktiven Unterstützung der irakischen Wahlkommission in der erfolgreichen Vorbereitung und Durchführung der Parlamentswahlen vom 7. März 2010 fokussierten die VN-Bemühungen auf die Begleitung des mehr als acht Monate dauernden Regierungsbildungsprozesses. Durch diese politische Pattstellung wurden 2010 auch die UNAMI-Initiativen zur Lösung von Binnengrenzkonflikten und offenen Verfassungsfragen weitgehend zum Stillstand gebracht. Durch den neuen Leiter von UNAMI, Ad Melkert, setzte ebenfalls eine stärkere Akzentuierung des VN-Engagements für Wiederauf- 
bau und langfristige Wirtschaftsentwicklung ein. Das Mandat von UNAMI wurde jährlich im August um weitere zwölf Monate verlängert, zuletzt bis 31. Juli 2011 (SR-Resolutionen 1883 (2009) bzw. 1936 (2010)).

In den beiden Jahren der österreichischen SR-Mitgliedschaft veranlasste die fragile Sicherheitslage den VN-SR zu mehreren Vorsitz- und Presseerklärungen. In diesen wurden die Terroranschläge im Irak auf das Schärfste verurteilt, darunter die Bombenanschläge auf irakische Ministerien und diplomatische Missionen am 19. August 2009 (SC/9733), dem sechsten Jahrestag des Anschlags auf das VN-Hauptquartier in Bagdad, sowie der Anschlag auf den Konvoi von SRSG Melkert im Oktober 2010 und die Anschlagsserie auf christliche und muslimische Glaubensstätten im November 2010 (SC/10081), in deren Verfolg sich insbesondere Österreich für Religionsfreiheit und den Schutz von religiösen Minderheiten einsetzte.

Am 15. Dezember 2010 hob der VN-SR in einer hochrangigen Sitzung unter Vorsitz von US-Vize-Präsident Joseph Biden und unter Teilnahme des Generalsekretärs für auswärtige Angelegenheiten Johannes Kyrle mit der Annahme von drei Resolutionen eine Reihe von Sanktionen gegen den Irak auf und beendete zwei Programme auf Basis von Mandaten gemäß Kapitel VII der VNSatzung. Mit SR-Resolution 1956 (2010) wurden die Privilegien und Immunitäten sowie der internationale Aufsichtsmechanismus für den Entwicklungsfonds für den Irak (DFI) letztmalig bis 30. Juni 2011 verlängert. SR-Resolution 1957 (2010) hob Sanktionen aus dem Jahr 1991 gegen Massenvernichtungswaffenprogramme des Irak und Restriktionen im Bereich der zivilen Nuklearentwicklung auf. Resolution 1958 (2010) leitete die Endabwicklung des Oilfor-Food Programms ein. Betreffend die Aufhebung von Sanktionen im Zusammenhang mit irakischen Massenvernichtungswaffen-programmen, die unter der letzten Mitgliedschaft von Österreich im VN-SR 1991/1992 beschlossen wurden, setzte sich Österreich für eine rasche Ratifizierung des Zusatzprotokolls des IAEO-Sicherheitskontrollabkommens und des CTBT ein.

Der VN-SR verlängerte die Finanzierung des Mandats des Hochrangigen Koordinators des VN-GS, Gennadij Tarasow, zur Aufklärung des Schicksals vermisster kuwaitischer Staatsangehöriger und Drittstaatsangehöriger sowie zur Repatriierung kuwaitischen Eigentums bis 30. Juni 2011.

In einer Presseerklärung vom 21. Dezember 2010 (SC/10138) begrüßte der VN-SR die Genehmigung der Bildung einer Regierung der nationalen Partnerschaft durch das irakische Parlament. An die politischen Führer des Landes wurde appelliert, die nationale Aussöhnung voranzutreiben.

\section{c) Asien}

\section{Demokratische Volksrepublik Korea}

In Folge des von der Demokratischen Volksrepublik Korea (DVRK) am 5. April 2009 durchgeführten Starts eines Flugkörpers, der von der überwie- 
genden Mehrheit der Staatengemeinschaft als Langstreckenrakete eingestuft wurde, und des am 25. Mai 2009 erfolgten zweiten Atomtests der DVRK seit 2006 sowie weiterer Raketenstarts im Mai beriet der VN-SR in mehreren Dringlichkeitssitzungen über die angespannte Lage auf der koreanischen Halbinsel. In seiner Vorsitzerklärung (S/PRST/2009/7) vom 13. April 2009 verurteilte der VN-SR den Start vom 5. April 2009 als Verstoß der DVRK gegen SR-Resolution 1718 (2006). In weiterer Folge nahm der VN-SR am 12. Juni 2009 einstimmig SR-Resolution 1874 (2009) an, mit welcher die gegen die DVRK bestehenden Sanktionen u.a. durch eine Ausdehnung des Waffenembargos und die Autorisierung von Inspektionen von Lieferungen in die und aus der DVRK, einschließlich Schiffskargoinspektionen auf Hoher See, sowie der Beschlagnahmung und Vernichtung sanktionierter Güter verschärft wurden. Dem mit SR-Resolution 1718 (2006) eingesetzten Sanktionenkomitee wurde durch SR-Resolution 1874 (2009) ein siebenköpfiges Expertenpanel zur Unterstützung bei der Umsetzung der Maßnahmen beigestellt. Das Sanktionenkomitee belegte in Folge der Raketenstarts und des Nukleartests weitere natürliche Personen mit Reiseverboten bzw. fror das Vermögen natürlicher und juristischer Personen ein.

In seiner Votumserklärung anlässlich der Annahme der SR-Resolution 1874 (2009) bekräftigte Österreich seine starke Verurteilung des nordkoreanischen Nukleartests als Verstoß gegen SR-Resolution 1718 (2006) und bezeichnete die konsensuale Entscheidung zur Verschärfung der Sanktionen als eine klare, angemessene und eindeutige Reaktion des VN-SR. Der in der Resolution enthaltene Aufruf an die DVRK, dem CTBT zum ehest möglichen Zeitpunkt beizutreten, geht auf einen österreichischen Vorschlag zurück. Die Aufdeckung mehrerer illegaler Lieferungen an die bzw. aus der DVRK seit der Ausweitung der Sanktionen und die Information des 1718-Sanktionenkomitees hiervon lassen auf die Wirksamkeit der beschlossenen Maßnahmen schließen. Das Expertenpanel des 1718-Sanktionenkomitees führte im März und im September 2010 mit den zuständigen österreichischen Behörden Arbeitsgespräche über die Umsetzung der SR-Resolutionen 1718 (2006) und 1874 (2009) und äußerte seine Zufriedenheit über die konstruktive österreichische Zusammenarbeit mit dem Sanktionenkomitee und dem Expertenpanel.

Im Jahr 2010 verschärften sich die Spannungen auf der koreanischen Halbinsel erneut aufgrund von zwei militärischen Zwischenfällen im Gelben Meer. Beim Untergang des südkoreanischen Marineschiffs Cheonan am 26. März 2010 kamen 46 Seeleute der Republik Korea ums Leben. Ein von der Republik Korea unter Beteiligung internationaler Experten publizierter Untersuchungsbericht hielt fest, dass die Cheonan durch Einwirkung eines Torpedos aus den Produktionsbeständen der DVRK versenkt worden sei. Am 14. Juni 2010 fanden zu den Untersuchungsergebnissen informelle interaktive Dialoge des VN-SR mit den Ständigen Vertretern der Republik Korea und der DVRK statt, die beiden Seiten Gelegenheit boten, ihre Sicht des Vorfalls 
darzulegen. Eine unter den P-5 mit Japan und der Republik Korea akkordierte Vorsitzerklärung (S/PRST/2010/13) wurde daraufhin am 9. Juli 2010 vom VN-SR angenommen. Darin wird der Angriff auf die Cheonan verurteilt, ohne auf die Frage der Urheberschaft einzugehen. Bundesminister Michael Spindelegger hatte bereits im Mai das Interesse Österreichs an einer raschen und geeinten Reaktion des VN-SR auf den Zwischenfall vom 26. März 2010 betont.

Der seitens der DVRK erfolgte Artilleriebeschuss der dem Hoheitsgebiet der Republik Korea unterstehenden Insel Yongpyong am 23. November 2010 stellte eine schwere Verletzung des Waffenstillstandsabkommens von 1953 dar. Bei dem Zwischenfall verloren zwei Soldaten und zwei Zivilisten der Republik Korea ihr Leben. Die DVRK behauptete, durch Seemanöver der Marine der Republik Korea im Gelben Meer provoziert worden zu sein. In zeitlicher Nähe zu dem Vorfall (am 20. November 2010) war ein Bericht eines amerikanischen Expertenteams (sog. „Hecker-Bericht“) über die Aktivitäten der DVRK hinsichtlich des Betriebs einer Urananreicherungsanlage und der Errichtung eines Leichtwasserreaktors veröffentlicht worden. Informelle Konsultationen im VN-SR dazu blieben ergebnislos.

Angesichts fortgesetzter Androhungen militärischer Gewaltanwendung durch die DVRK für den Fall der Abhaltung der von der Republik Korea für 18.-21. Dezember 2010 angekündigten Militärübungen beantragte die Russische Föderation für 19. Dezember 2010 eine Dringlichkeitssitzung des VN-SR. Trotz mehrstündiger Verhandlungen in informellen Konsultationen konnte keine Einigung auf eine Presseerklärung des VN-SR erzielt werden. Im Anschluss daran fand eine ,private“ Sitzung des VN-SR statt, an der die Ständigen Vertreter der Republik Korea und der DVRK teilnahmen. Österreich hatte sich dafür eingesetzt, dass beiden Seiten die Möglichkeit geboten werden müsse, sich zu äußern. In der Sitzung verurteilte auch Österreich neben anderen SR-Mitgliedern den Artillerieangriff der DVRK vom 23. November. Ferner rief Österreich beide Seiten zu Besonnenheit und zur Einhaltung des Waffenstillstandsabkommens auf und appellierte, eine Lösung des Konflikts ausschließlich auf dem Verhandlungsweg zu suchen und am Ziel einer nuklearwaffenfreien koreanischen Halbinsel festzuhalten.

\section{Afghanistan}

Der Unterstützungsmission der VN in Afghanistan (UNAMA) kommt eine Führungsrolle bei der Koordination und Umsetzung der internationalen zivilen Aktivitäten in Afghanistan zu. Das Mandat der Mission wurde 2009/2010 zweimal verlängert, zuletzt mit SR-Resolution 1917 (2010) bis 23. März 2011. Der VN-SR beriet auf Basis vierteljährlicher Berichte des VN-GS über die aktuellen Entwicklungen in Afghanistan. Österreich setzte sich in den Verhandlungen zu den Mandatsverlängerungen von UNAMA für eine Stärkung des Mandats hinsichtlich der Rechte von Frauen und Kindern und für die 
Erarbeitung von „Benchmarks“ zur Bewertung der Fortschritte bei der Umsetzung des Mandats in diesen Bereichen ein. In den vierteljährlichen Debatten zur Situation in Afghanistan verwies Österreich konsequent auf die problematische Menschenrechtslage und den mangelnden Schutz der Zivilbevölkerung im bewaffneten Konflikt und thematisierte u.a. Übergriffe gegen Frauen und Kinder, die geschlechterdiskriminierende nationale Gesetzgebung sowie die unzureichenden Maßnahmen zur Bekämpfung der Straflosigkeit.

Darüber hinaus fanden 2009/10 zusätzliche Konsultationen und Briefings zu Afghanistan statt. Am 15. Juli 2009 nahm der VN-SR eine Vorsitzerklärung (S/PRST/2009/21) im Hinblick auf die Präsidentschaftswahlen vom 20. August 2009 an, in der die Bedeutung freier, fairer und glaubwürdiger Wahlen betont wird. Auf eine Anschlagsserie gegen öffentliche und internationale Einrichtungen (darunter ein Angriff auf ein Gästehaus der VN in Kabul am 28. Oktober 2009, bei dem mehrere VN-Mitarbeiter ums Leben kamen) sowie auf die Bekanntgabe des offiziellen Wahlergebnisses reagierte der VN-SR mit Presseerklärungen (SC/9593, SC/9735, SC/9778, SC/9784).

Die Londoner Konferenz vom 28. Jänner 2010 und die Abhaltung der ersten internationalen Afghanistan-Konferenz in Kabul am 20. Juli 2010 markierten wichtige Schritte im Prozess zur graduellen Übertragung der Verantwortung für Sicherheit, Regierungsführung und wirtschaftliche Entwicklung an die afghanische Regierung. Gemeinsam mit dem „Liechtenstein Institute on SelfDetermination“ der Princeton Universität organisierte das BMeiA unter Leitung des Österreichers Professor Wolfgang Danspeckgruber von 25.-28. Februar 2010 in Baden eine Implementierungskonferenz zur Umsetzung der Ergebnisse von London („LISD Afghanistan Implementation Colloquium“). Ziel des hochrangig besetzten Treffens war es, die Themen der Londoner Konferenz weiter zu vertiefen und einen Beitrag zur Vorbereitung der Folgekonferenz in Kabul zu leisten. Der VN-SR rief in Presseerklärungen vom 5. Februar 2010 (SC/9858) und vom 23. Juli 2010 (SC/9992) zur zügigen Umsetzung der in London und Kabul formulierten Bekenntnisse und Vorhaben auf.

Der bereits in London intensiv erörterte Versöhnungs- und Reintegrationsprozess gewann im Laufe des Jahres 2010 an Dynamik. Kurz vor der Londoner Konferenz war im Rahmen des 1267-Sanktionenkomitees (Al-Qaida/Taliban) des VN-SR die Streichung von fünf ehemaligen hochrangigen Talibanvertretern von der Sanktionenliste erfolgt. Im Rahmen der Reise des VN-SR nach Afghanistan von 21.-24. Juni 2010, an der VertreterInnen aller 15 SRMitglieder teilnahmen, kam klar zum Ausdruck, dass Präsident Hamid Karzai und die Mehrzahl afghanischer Spitzenpolitiker immer stärker auf eine Aussöhnung mit gemäßigten Taliban drängen.

Anlässlich der offenen Debatte zu Afghanistan am 22. Dezember 2010 nahm der VN-SR eine Presseerklärung (SC/10143) an, in der die Verkündigung des Endergebnisses der Parlamentswahlen vom 18. September 2010 als Meilen- 
stein im politischen Prozess in Afghanistan begrüßt wird. In der Debatte wurden auch die Fortschritte bei der Umsetzung des bei der Kabuler Konferenz angekündigten Arbeitsprogramms der afghanischen Regierung gewürdigt. Österreich wies zum wiederholten Male darauf hin, dass Kriterien wie die Einhaltung der Menschen- und Frauenrechte im Versöhnungsprozess und bei der Reintegration ehemaliger Taliban Berücksichtigung finden müssen. Die gute Zusammenarbeit zwischen Afghanistan sowie UNAMA und dem 1267-Sanktionenkomitee (Al-Qaida/Taliban) wurde von Österreich positiv hervorgehoben. Österreich unterstrich die Notwendigkeit einer Intensivierung der regionalen Anstrengungen zur Eindämmung des Drogenanbaus und -handels in der Region in Kooperation mit UNODC.

Die Autorisierung der Internationalen Sicherheitsbeistandstruppe (ISAF) wurde zweimal um jeweils zwölf Monate verlängert, zuletzt mit SR-Resolution 1943 (2010) bis 13. Oktober 2011. Österreich konnte in dieser Resolution einige wichtige Ergänzungen verankern, wie etwa einen Verweis auf die Problematik der Rekrutierung von Kindersoldaten durch die Taliban sowie die Tötung von Kindern im Zuge des Konflikts und die Hervorhebung der Bedeutung des Monitorings und der Berichterstattung über die Situation von Zivilisten.

\section{Kirgisistan}

Im Anschluss an den Sturz der Regierung Bakijew im April 2010 sowie anlässlich der gewaltsamen Auseinandersetzungen zwischen ethnischen Kirgisen und der usbekischen Minderheit in Südkirgisistan im Juni befasste sich der VN-SR mehrmals mit den Entwicklungen in der zentralasiatischen Republik. Die Situation in Kirgisistan wurde dabei nicht offiziell auf die Tagesordnung des VN-SR gesetzt.

Der VN-GS informierte den VN-SR am 15. April 2010 über seinen Besuch in Zentralasien von 1.-7. April 2010 und ging dabei auch auf die Situation in Kirgisistan ein. Er informierte über die erzielte Einigung zur Ausreise von Präsident Kurmanbek Bakijew und forderte Kirgisistan zur Rückkehr zu Rechtsstaatlichkeit auf. Die SR-Mitglieder begrüßten v.a. die effektive Kooperation der VN mit der EU und der Organisation für Sicherheit und Zusammenarbeit in Europa (OSZE) bei der Bewältigung der Krise. Österreich gab seiner Hoffnung auf die Abhaltung freier und fairer Wahlen Ausdruck, die von der internationalen Gemeinschaft unterstützt werden sollten.

Am 14. Juni 2010 berichtete der VN-Untergeneralsekretär (UGS) für politische Angelegenheiten, Lynn Pascoe, dem VN-SR in einem geschlossenen Briefing über die jüngsten Entwicklungen in Kirgisistan. UGS Pascoe zeigte sich besorgt über die Anstiftungen zu ethnischer Gewalt und informierte über die angelaufenen humanitären Hilfsmaßnahmen der VN für die rund 75.000 Flüchtlinge. Der VN-SR konnte sich auf Bemerkungen des SR-Vorsitzes gegenüber der Presse einigen, in denen Besorgnis über die dramatische 
Lage sowie Unterstützung für die Bemühungen des VN-GS und der involvierten Regionalorganisationen geäußert wird. Außerdem verurteilte der VN-SR die fortdauernde Gewalt, betonte die Dringlichkeit von humanitärer Hilfe und rief zur Rückkehr zu Rechtsstaatlichkeit und friedlicher Zusammenarbeit auf.

In den geschlossenen Konsultationen des VN-SR am 24. Juni 2010 wies der beigeordnete VN-Generalsekretär (ASG) für politische Angelegenheiten, Oscar Fernández-Taranco, auf die Gefahr hin, dass angesichts der instabilen Lage und der enormen Spannungen bereits ein kleiner Zwischenfall die Situation neuerlich zum Eskalieren bringen könnte. Die VN würden Unterstützung bei der Abhaltung des für 27. Juni geplanten Verfassungsreferendums leisten und seien um einen inklusiven Prozess bemüht. Unter den SR-Mitgliedern herrschte Einigkeit über die Bedeutung der engen Zusammenarbeit der VN mit der OSZE, der EU, der Organisation des Vertrags über Kollektive Sicherheit (CSTO) und anderen regionalen Akteuren. Als einziges SR-Mitglied verwies Österreich zudem auf den gemeinsamen Aufruf der Sonderberater des VN-GS für die Verhütung von Völkermord und für die „Responsibility to Protect“", Francis Deng und Edward Luck, wonach die internationale Gemeinschaft Schritte setzen müsse, um die kirgisische Regierung dabei zu unterstützen, ihrer Schutzverantwortung gegenüber der eigenen Bevölkerung nachzukommen. Die Konsultationen des VN-SR blieben ohne konkretes Ergebnis.

In seinem halbjährlichen Briefing über die Arbeit des VN-Regionalzentrums für vorbeugende Diplomatie für Zentralasien (UNRCCA) am 5. August 2010 informierte SRSG Miroslav Jenča den VN-SR auch über die Aktivitäten des UNRCCA zur Bewältigung der Krise in Kirgisistan. In Zusammenarbeit mit der EU, der OSZE und der CSTO sei es UNRCCA gelungen, rasch auf die Gewalt in Kirgisistan zu reagieren. Das Regionalzentrum werde im engen Kontakt mit den Nachbarstaaten vertrauensbildende Maßnahmen und den politischen Dialog fördern und Kirgisistan bei der Durchführung der Parlamentswahlen im Oktober unterstützen. In der vom VN-SR angenommenen Presseerklärung (SC/10005) wird die Rolle des UNRCCA im Zusammenhang mit den Ereignissen in Kirgisistan begrüßt. Österreich unterstrich, dass eine langfristige Lösung und Stabilisierung der Lage in Kirgisistan nur durch den Aufbau demokratischer Institutionen erreicht werden könne. Im Hinblick auf die interethnische Aussöhnung und ein besseres Verständnis für die Ursachen des Konflikts unterstützte Österreich gemeinsam mit anderen SRMitgliedern die Einsetzung einer internationalen Untersuchungskommission unter Einbindung der Expertise des Büros der VN-Hochkommissarin für Menschenrechte.

\section{Nepal}

Der Friedensprozess in Nepal war im Berichtszeitraum von zunehmender Stagnation und einer Verhärtung der Fronten zwischen den politischen Par- 
teien gekennzeichnet. Das für die Verlängerung des Mandats der Politischen Mission der VN in Nepal (UNMIN) erforderliche Ersuchen der Regierung von Nepal an den VN-GS erfolgte jeweils erst kurz vor Auslaufen des Mandats. Hauptaufgabe der Mission in den Jahren 2009/10 war die Überwachung des Umgangs mit den Waffen und des bewaffneten Personals der ehemaligen Bürgerkriegsparteien. Die mit SR-Resolution 1864 (2009) beschlossene Reduzierung der Personalstärke von UNMIN ging einher mit der Ablöse des SRSG und Leiters der Mission, Ian Martin, durch Karin Landgren, die die Leitung von UNMIN ab Februar 2009 im Rang einer Beauftragten des VN-GS (RSG) ausübte. Österreich beteiligte sich nach einer Unterbrechung ab April 2009 erneut mit zwei Militärexperten („Arms Monitors“) an UNMIN und unterstützte die Arbeit des Büros der VN-Hochkommissarin für Menschenrechte in Kathmandu durch den Einsatz einer „Junior Professional Officer" von August 2007 bis November 2009.

Auf Basis der Berichte des VN-GS über die Umsetzung des Mandats von UNMIN befasste sich der VN-SR 2009/10 insgesamt zehnmal mit der Situation in Nepal. Die Debatte am 5. Mai 2009 stand im Zeichen der Regierungskrise nach dem Rücktritt von Premierminister Pushpa Kamal Dahal Prachanda und dem Austritt der maoistischen Partei aus der Regierung. Der VN-SR rief in einer Vorsitzerklärung (S/PRST/2009/12) die nepalesische Regierung und alle politischen Parteien zur Fortsetzung der Zusammenarbeit auf und bekräftige seine Unterstützung für den Friedensprozess. Im Hinblick auf die Ausarbeitung einer neuen nepalesischen Verfassung unterbreitete Österreich das Angebot, seine Expertise in diesem Bereich zur Verfügung zu stellen. Weiters betonte Österreich die Notwendigkeit einer raschen Entlassung der Kindersoldaten im Einklang mit internationalen Standards und äußerte Bedenken hinsichtlich der Straflosigkeit. Auf österreichischen Vorschlag konnte in der SR-Resolution 1879 (2009) erstmals ein Absatz verankert werden, der das Problem der Straflosigkeit anspricht und die Bedeutung des Schutzes und der Förderung der Menschenrechte im Wege des Kapazitätenaufbaus von unabhängigen nationalen Institutionen betont.

Am 6. November 2009 wies RSG Landgren darauf hin, dass mangels Fortschritten im Friedensprozess eine Beendigung der Mission mit Jänner 2010 nicht realistisch erscheine. Als größte Herausforderungen erwiesen sich die Integration der maoistischen Kämpfer in die staatliche Armee und die Ausarbeitung einer föderalistischen Verfassung. In diesem Zusammenhang organisierte Ôsterreich im November 2009 in Wien ein Seminar für eine hochrangige Delegation nepalesischer Verfassungsjuristen.

Im Lichte der weiter verschärften Konfrontation zwischen den politischen Parteien ab dem Frühjahr 2010 und der ungeklärten Frage der Übergabe der Monitoring-Aufgaben hinsichtlich der Waffenarsenale und des bewaffneten Personals der Bürgerkriegsparteien an nepalesische Akteure nahm der VN-SR drei weitere Resolutionen zur Verlängerung von UNMIN an. Mit SR-Resolu- 
tion 1909 (2010) wurde UNMIN auch konkret zur Vorbereitung des Abzugs aufgefordert.

Am 7. September 2010 informierte RSG Landgren den VN-SR über die zu Sorge Anlass gebenden Entwicklungen in Nepal nach dem Rücktritt von Premierminister Madhav Kumar Nepal Ende Juni. Mit der Verlängerung der Frist zur Ausarbeitung einer neuen Verfassung bis zum 28. Mai 2011 konnte vorläufig der Fortbestand des Friedensprozesses gesichert werden. Im August 2010 hatte Österreich zur Unterstützung des Verfassungsgebungsprozesses ein weiteres Seminar für nepalesische Verfassungsexperten in Wien und den Bundesländern durchgeführt.

Mit SR-Resolution 1939 (2010) wurde das Mandat von UNMIN im Einklang mit einem entsprechenden Ersuchen der nepalesischen Regierung ein letztes Mal um vier Monate bis 15. Jänner 2011 verlängert. Im Oktober und Dezember 2010 reiste UGS Pascoe zweimal zu hochrangigen Vermittlungsgesprächen nach Nepal und berichtete im Anschluss daran dem VN-SR. Am 20. Oktober 2010 nahm der VN-SR eine Presseerklärung (SC/10062) an, in der Unterstützung für die Vermittlungsbemühungen von UGS Pascoe zum Ausdruck gebracht und die Parteien in Nepal zu verstärkter Kooperation aufgefordert werden. Von 21.-26. November 2010 unternahm die Arbeitsgruppe des VN-SR zu Kindern und bewaffneten Konflikten ihre erste Feldmission seit ihrer Einrichtung nach Nepal (nähere Ausführungen siehe Kapitel III.4).

\section{Myanmar}

Die innenpolitische Lage in Myanmar war 2009/10 Gegenstand mehrerer Konsultationen des VN-SR, in denen die SR-Mitglieder u.a. Briefings des VN-GS und der Sonderberater des VN-GS (SA) für Myanmar, Ibrahim Gambari bzw. UGS Vijay Nambiar (ab 2010) über deren Gute Dienste erhielten.

Am 20. Februar 2009 berichtete SA Gambari dem VN-SR über seine kurz zuvor erfolgte Mission nach Myanmar. In Reaktion auf die Verhaftung und Anklageerhebung gegen Friedensnobelpreisträgerin Daw Aung San Suu Kyi vom 14. Mai 2009 nahm der VN-SR am 22. Mai eine Presseerklärung an, in welcher er seine Besorgnis über die politischen Auswirkungen dieser Maßnahmen äußerte. Am 13. Juli 2009 informierte der VN-GS den VN-SR im Rahmen einer öffentlichen Sitzung über seinen Besuch in Myanmar am 3./4. Juli. Am 11. August 2009 trat der VN-SR anlässlich der Verurteilung von Daw Aung San Suu Kyi zu weiteren 18 Monaten Hausarrest zu geschlossenen Konsultationen zusammen. Österreich forderte gemeinsam mit der Mehrheit der SR-Mitglieder die Freilassung von Daw Aung San Suu Kyi sowie aller politischen Gefangenen und trat für eine rasche, geschlossene und deutliche Antwort des VN-SR auf die Verurteilung der Friedensnobelpreisträgerin ein. Am 13. August 2009 einigte sich der VN-SR auf eine Presseerklärung, in der ernste Bedenken wegen des Urteils gegen Aung San Suu Kyi ausgedrückt und die Forderung nach der Freilassung aller politischen Gefangenen bekräftigt wurden. 
Am 24. März 2010 informierte der Kabinettschef des VN-GS, UGS Vijay Nambiar, in seiner Funktion als neuer SA für Myanmar über die Situation nach Veröffentlichung der Wahlgesetze für die Parlamentswahlen im November. Die SR-Mitglieder äußerten breite Unterstützung für die Guten Dienste der VN und betonten die Notwendigkeit, auf einen inklusiven politischen Prozess hinzuwirken. Nach den Wahlen vom 7. November 2010 sowie der darauf folgenden Entlassung von Daw Aung San Suu Kyi aus dem Hausarrest trat der VN-SR am 18. November 2010 neuerlich zu geschlossenen Konsultationen mit UGS Nambiar zusammen. Österreich wies gemeinsam mit der Mehrheit der SR-Mitglieder darauf hin, dass die Wahlen nicht demokratischen Standards entsprochen hätten, begrüßte das Ende des Hausarrests von Daw Aung San Suu Kyi und forderte die Freilassung aller weiteren politischen Gefangenen. Am 6. Dezember 2010 berichtete UGS Nambiar dem VN-SR in geschlossenen Konsultationen über seinen ersten offiziellen Besuch in Myanmar von 27.-28. November 2010. Österreich sprach sich für eine aktive Fortsetzung der Guten Dienste der VN sowie einen weiteren Besuch von UGS Nambiar in Myanmar noch vor der Bildung einer neuen Regierung aus.

\section{Timor-Leste}

Vor dem Hintergrund der Allparteiengespräche zwischen 1995 und 1998 in Stadtschlaining und auf Schloss Krumbach sind Österreich der innertimoresische Aussöhnungsprozess und die weitere Entwicklung Timor-Lestes ein besonderes Anliegen. Die Lage in Timor-Leste hat sich in den Jahren 2009/10 nicht zuletzt aufgrund der Präsenz der Integrierten Mission der VN in TimorLeste (UNMIT) sowie einer multinationalen Friedenstruppe unter australischer Führung weiter stabilisiert. Das Mandat von UNMIT wurde zweimal um jeweils ein Jahr verlängert, zuletzt mit SR-Resolution 1912 (2010) bis 26. Februar 2011. Österreich brachte sich in den Mandatsverhandlungen erfolgreich mit Vorschlägen zu Menschenrechten, zur Beendigung der Straflosigkeit für Menschenrechtsverletzungen und zur Berücksichtigung der Rechte und Bedürfnisse von Frauen im Rahmen der Sicherheitssektorreform ein.

Der VN-SR befasste sich 2009/10 auf der Grundlage der halbjährlichen Berichte des VN-GS in vier offenen Debatten mit der Situation in TimorLeste. In der Debatte am 19. Februar 2009 unter Beteiligung des timoresischen Präsidenten José Ramos-Horta äußerte sich der VN-SR einhellig positiv zur Verbesserung der Sicherheitslage und zu den von Timor-Leste erreichten Fortschritten bei der Lösung der den vergangenen Konflikten zugrunde liegenden Probleme. Österreich hob die Stärkung der Rechtsstaatlichkeit als eine Voraussetzung für nachhaltige Stabilität hervor und begrüßte die aktive Mitarbeit von Timor-Leste im Rahmen der von Österreich ins Leben gerufenen „Group of Friends on the Rule of Law“.

Anlässlich des 10. Jahrestags des Referendums über die Unabhängigkeit von Timor-Leste vom 30. August 1999 einigte sich der VN-SR am 27. August 2009 
auf Bemerkungen des SR-Vorsitzes gegenüber der Presse, in denen die Bemühungen Timor-Lestes um Frieden, Stabilität und Entwicklung gewürdigt wurden und - auf Anregung Österreichs - auch die Opfer der Gewalttaten des Jahres 1999 Erwähnung fanden.

In der offenen Debatte des VN-SR am 23. Oktober 2009 wurde der friedliche Verlauf der Lokalwahlen vom 9. Oktober 2009 als wichtiger Fortschritt im Friedensprozess gewürdigt. Österreich hob auch die Rückkehr Binnenvertriebener als positive Entwicklung hervor, wies aber zugleich auf noch anstehende Herausforderungen wie die Beendigung der Straflosigkeit oder die Armutsbekämpfung und Schaffung von Beschäftigungsmöglichkeiten hin. Die neue SRSG Ameerah Haq (Nachfolgerin von SRSG Atul Khare) zeigte sich in der offenen Debatte am 23. Februar 2010 zuversichtlich, dass der laufende Prozess der Übergabe der Polizeiaufgaben an die timoresische Polizei bald abgeschlossen werden könnte, wenn die Bemühungen zur Erfüllung der vereinbarten Kriterien in konzentrierter Form fortgesetzt würden. In Ergänzung zu den Schwerpunkten früherer Wortmeldungen bot Österreich Unterstützung für die Bemühungen der timoresischen Armee zum Aufbau von Kapazitäten im Bereich des Katastrophenschutzes und der Beteiligung an FEO der VN an. Präsident Ramos-Horta wurde anlässlich seines Besuchs in Österreich von 2.-4. November 2010 über die Bereitschaft des BMLVS informiert, Ausbildungsplätze für Offiziere aus Timor-Leste zum Training in der internationalen Friedenssicherung zur Verfügung zu stellen.

Hinsichtlich der Zukunft von UNMIT informierte SRSG Haq den VN-SR in der offenen Debatte am 19. Oktober 2010 über die Einsetzung eines Mechanismus, in dessen Rahmen die Regierung sowie UNMIT gemeinsam die Planung für den Übergang von UNMIT zu einer anderen Form der VN-Präsenz im Jahr 2012 vornehmen sollen. Für 2012 sind allgemeine Wahlen geplant, die auch einen Test für die Fähigkeiten der timoresischen Polizeikräfte darstellen werden.

\section{Sri Lanka}

In der ersten Jahreshälfte 2009 befasste sich der VN-SR angesichts der Eskalation des bewaffneten Konflikts zwischen den Liberation Tigers of Tamil Eelam (LTTE) und den srilankischen Streitkräften mehrmals mit der Situation in Sri Lanka. Dies erfolgte großteils im Rahmen der von Österreich mitinitiierten informellen interaktiven Diskussionen, die es dem VN-SR ermöglichten, sich mit der prekären humanitären Situation in Sri Lanka auseinander zu setzen, ohne Sri Lanka formell auf die Tagesordnung des VN-SR zu setzen. Der Konflikt in Sri Lanka zeigte die Bedeutung der konsequenten Umsetzung der österreichischen Prioritäten im VN-SR.

Der UGS für humanitäre Angelegenheiten, John Holmes, informierte den VN-SR am 27. Februar 2009 in geschlossenen Konsultationen über seinen Besuch in Sri Lanka von 19.-21. Februar 2009 in seiner Funktion als VN- 
Nothilfekoordinator. Er äußerte große Sorge in Bezug auf das Schicksal der tausenden im Kampfgebiet eingeschlossenen Zivilisten, forderte deren Freilassung durch die LTTE sowie die Verhängung einer temporären Waffenruhe. Neben VN-GS Ban Ki-moon waren es anfänglich v.a. die nicht-ständigen SRMitglieder Mexiko, Costa Rica und Österreich sowie Frankreich und Großbritannien als ständige Mitglieder, die den VN-SR zu einer Befassung mit der sich zuspitzenden Situation drängten.

Am 26. März trat der VN-SR erstmals zu einer informellen Diskussion unter Beteiligung des Ständigen Vertreters von Sri Lanka zusammen, um angesichts der weiteren Verschlechterung der humanitären Situation einen neuerlichen Bericht von UGS Holmes zu erhalten. Am 22. April 2009 berichtete der Kabinettschef des VN-GS, UGS Vijay Nambiar, dem VN-SR über seine Vermittlungsmission von 16.-17. April 2009. Im Zuge des Treffens einigten sich die SR-Mitglieder auf Bemerkungen des SR-Vorsitzes gegenüber der Presse, in denen die LTTE zur sofortigen Niederlegung ihrer Waffen und beide Konfliktparteien zur Einhaltung des humanitären Völkerrechts sowie zur Schaffung eines Zugangs für humanitäre HelferInnen aufgefordert wurden. In weiteren Bemerkungen des SR-Vorsitzes gegenüber der Presse vom 24. April 2009 wurde die Regierung von Sri Lanka aufgefordert, einem vom VN-GS in die Konfliktzone zu entsendenden humanitären Team jegliche erforderliche Unterstützung zu gewähren. Am 30. April 2009 berichtete UGS Holmes dem VN-SR über seine Mission nach Sri Lanka von 25.-27. April 2009. Mit neuerlichen Bemerkungen des SR-Vorsitzes gegenüber der Presse bekräftigten die SR-Mitglieder ihren Aufruf an die Regierung, den humanitären Zugang zur Konfliktzone zu ermöglichen.

Am 11. Mai 2009 fand in New York im Anschluss an ein informelles Treffen von zehn SR-Mitgliedern mit dem VN-Büro für die Koordinierung humanitärer Angelegenheiten (OCHA) sowie mehreren internationalen NRO eine gemeinsame Pressekonferenz von Bundesminister Michael Spindelegger, dem britischen Außenminister David Miliband und dem französischen Außenminister Bernard Kouchner zur Situation in Sri Lanka statt. Dabei betonte Bundesminister Michael Spindelegger die Verantwortung der srilankischen Regierung für den Schutz ihrer Bevölkerung, forderte den Zugang unabhängiger BeobachterInnen zu den Lagern für die Binnenvertriebenen und hob die Notwendigkeit einer politischen Lösung des Konflikts hervor.

Am 13. Mai 2009 einigte sich der VN-SR auf gemeinsame Initiative von Frankreich, Großbritannien und Österreich erstmals auf eine formelle Presseerklärung (SC/9659) zu Sri Lanka, in der alle Parteien dringend zur Einhaltung des humanitären Völkerrechts angehalten, die LTTE zur Niederlegung der Waffen und die srilankische Regierung zur Erleichterung des humanitären Zugangs und der Evakuierung der eingeschlossenen Zivilisten aufgerufen wurden. Weiters wurde die Bedeutung einer langfristigen politischen Lösung unter Berücksichtigung der Bedürfnisse aller Bevölkerungsgruppen in Sri Lanka betont. Am 5. Juni 2009 informierte der VN-GS den VN-SR nach 
dem Ende der Kampfhandlungen im Rahmen einer informellen Sitzung über seinen Besuch in Sri Lanka am 22. und 23. Mai 2009. Österreich betonte die Notwendigkeit einer Verbesserung der humanitären Situation in den Lagern der Binnenvertriebenen, einer unabhängigen Untersuchung von Verletzungen der Menschenrechte und des humanitären Völkerrechts sowie der Einleitung eines nationalen Versöhnungsprozesses.

Obwohl die Regierung von Sri Lanka ihre militärischen Operationen fortsetzte, gehen viele Beobachter davon aus, dass die srilankischen Streitkräfte angesichts der Diskussionen im VN-SR (und sonstiger VN-Aktivitäten) auf die Sicherheitsbedürfnisse der Zivilisten im Konfliktgebiet größere Rücksicht genommen haben.

\section{Pakistan}

Im Gefolge der Ermordung der ehemaligen pakistanischen Premierministerin Benazir Bhutto bei einer Wahlveranstaltung in Rawalpindi am 27. Dezember 2007 hatte die pakistanische Regierung die VN im November 2008 um Einsetzung einer internationalen Untersuchungskommission zu dem Attentat ersucht. Die vom VN-GS eingesetzte Kommission unter der Leitung des Ständigen Vertreters Chiles bei den VN in New York, Heraldo Muñoz, nahm am 1. Juli 2009 ihre Arbeit auf. Am 15. April 2010 legte die Kommission ihren Bericht der pakistanischen Regierung sowie dem VN-SR zur Information vor. Dem Mandat der Kommission entsprechend beschränkt sich der Bericht auf die objektive Darlegung der Umstände des Attentats und bestätigt die Feststellung von Scotland Yard, dass Benazir Bhutto durch die Explosion eines Sprengsatzes getötet wurde. Die Feststellung der strafrechtlichen Verantwortlichkeit obliegt den pakistanischen Behörden.

\section{Regionale Themen}

Das VN-Regionalzentrum für vorbeugende Diplomatie für Zentralasien (UNRCCA) wurde im Jahr 2007 auf Initiative der fünf zentralasiatischen Staaten als politische Mission der VN in Aschgabat eingerichtet. Ziel des UNRCCA, das 2008 seine Tätigkeit aufgenommen hat, ist die Unterstützung der Länder Zentralasiens beim Aufbau ihrer Kapazitäten für Konfliktprävention und im Hinblick auf eine gemeinsame Herangehensweise an grenzüberschreitende Bedrohungen wie Terrorismus, Drogenhandel, organisierte Kriminalität und Umweltzerstörung. Auch die verstärkte Zusammenarbeit bei der Nutzung von Wasser- und Energieressourcen zählt zu den Aufgaben des UNRCCA.

In seiner Funktion als Leiter des UNRCCA berichtete SRSG Jenča dem VN-SR 2009/10 halbjährlich in geschlossenen Konsultationen über die Aktivitäten des Regionalzentrums. Im Rahmen des Briefings vom 19. Juni 2009 unterstri- 
chen die SR-Mitglieder die Notwendigkeit der Kooperation mit anderen internationalen und regionalen Akteuren. Österreich forderte dazu auf, das Engagement von UNRCCA und dessen Rolle im Wasser- und Energiekonflikt weiter auszubauen. In den geschlossenen Konsultationen vom 14. Jänner 2010 ging SRSG Jenča auf die laufenden Arbeiten zur Bekämpfung von Drogenhandel, organisiertem Verbrechen und Terrorismus (in enger Zusammenarbeit mit UNODC) ein und verwies auf das neue Liaisonarrangement zwischen UNRCCA und UNAMA. Im Zusammenhang mit der Drogenbekämpfung hob Jenča das österreichische Engagement im Bereich Grenzverwaltung im Rahmen der Zentralasiatischen Grenzsicherheitsinitiative (CABSI) hervor. In seinem Briefing vom 5. August 2010 informierte SRSG Jenča den VN-SR insbesondere über die Aktivitäten des UNRCCA in Zusammenarbeit mit der EU, OSZE und der CSTO bei der Bewältigung der Krise in Kirgisistan (für nähere Ausführungen siehe Kapitel II.c.3).

Als Ergebnis der Konsultationen zu UNRCCA nahm der VN-SR jeweils eine Presseerklärung (SC/9686, SC/9843, SC/10005) an, in der die Unterstützung für die Anstrengungen des Regionalzentrums hinsichtlich der Förderung des Dialogs und der Hilfestellung für die Regierungen Zentralasiens bei der Bewältigung der vielfältigen Herausforderungen in der Region ausgedrückt wird.

\section{d) Europa}

\section{Kosovo}

Insgesamt sieben Mal behandelte der VN-SR unter Beteiligung von Vertretern Serbiens und des Kosovo die vierteljährlichen Berichte des VN-GS zur VNMission im Kosovo (UNMIK). Nach Abschluss der Rekonfigurierung von UNMIK als Reaktion auf die veränderte Situation in Folge der Unabhängigkeitserklärung des Kosovo und der damit verbundenen Übergabe aller Aufgaben im Bereich der Rechtsstaatlichkeit inklusive der Polizeiaufgaben an die Rechtsstaatlichkeitsmission der EU (EULEX Kosovo) haben sich die Aufgaben von UNMIK deutlich reduziert. Während die Lage im Kosovo weitgehend stabil blieb, konnten nur wenige Fortschritte in der praktischen Zusammenarbeit zwischen Serbien und dem Kosovo in den Bereichen Justiz, Zoll und kulturelles Erbe erzielt werden. Die Integration des Nordens in die staatlichen kosovarischen Strukturen erwies sich nach wie vor als schwierig. Seitens der kosovarischen Regierung ließ sich eine abnehmende Bereitschaft, mit UNMIK zusammenzuarbeiten, erkennen. In der letzten Sitzung zu Kosovo/UNMIK während österreichischer SR-Mitgliedschaft am 12. November 2010 wurde auch die von der VN-GV am 9. September 2010 angenommene Resolution (A/RES/64/298) über das Rechtsgutachten des Internationalen Gerichtshofs (IGH) zur Frage der Rechtmäßigkeit der kosovarischen Unabhängigkeitserklärung (in der die Bereitschaft der EU, einen Dialog zwischen den Parteien zu unterstützen, begrüßt wird) thematisiert. 


\section{Europa}

Am 6. Juli 2010 befasste sich der VN-SR mit der Sicherheitslage im Kosovo. Serbien hatte um diese dringliche Sitzung mit der Begründung ersucht, dass sich die Sicherheitslage verschlechtert habe, nachdem am 2. Juli 2010 während Demonstrationen in Nord-Mitrovica gegen die Eröffnung eines Verwaltungsbüros durch die Explosion eines Sprengsatzes eine Person getötet und zehn weitere verletzt worden waren. Die Leiter von UNMIK sowie der EUMission EULEX informierten den VN-SR über die faktische Lage vor Ort und betonten, dass trotz der Spannungen keine Destabilisierung der Lage gegeben sei.

Österreich hat sich in allen Debatten konstant für eine Zusammenarbeit aller Seiten mit der internationalen Gemeinschaft, für eine pragmatische Haltung der serbischen und kosovarischen Institutionen sowie für eine Integration des Nordens ausgesprochen und die europäische Perspektive Serbiens und des Kosovo betont. Weiters hat Österreich regelmäßig die Notwendigkeit von Reformen insbesondere in den Bereichen der Rechtsstaatlichkeit und der guten Regierungsführung, sowie der Bekämpfung der organisierten Kriminalität und der Korruption unterstrichen. Österreich hat sich auch von Beginn an mit Erfolg dafür eingesetzt, dass sowohl ein Vertreter Serbiens als auch ein Vertreter des Kosovo vor dem VN-SR sprechen konnten.

\section{Bosnien und Herzegowina}

Am 25. März 2009 nahm der VN-SR einstimmig SR-Resolution 1869 (2009) an, mit der er der Bestellung des österreichischen Diplomaten Botschafter Valentin Inzko zum neuen Hohen Vertreter in Bosnien und Herzegowina zustimmte. Der VN-SR verlängerte das Mandat der militärischen Operation der EU in Bosnien und Herzegowina (EUFOR Althea) zweimal um jeweils 12 Monate, zuletzt mit SR-Resolution 1948 (2010). In seiner Eigenschaft als europäisches SR-Mitglied war Österreich Mitglied der „Contact and Drafting Group“ für diese Resolutionen. Insgesamt vier Mal berichtete Botschafter Inzko dem VN-SR in seiner Eigenschaft als Hoher Vertreter über die Entwicklungen in Bosnien und Herzegowina.

Gegenstand der Diskussionen des VN-SR waren u.a. die weiterhin schwierige politische Situation in Bosnien und Herzegowina, mangelnde Fortschritte in der Durchführung von Reformen zur euro-atlantischen Integration sowie zur Schließung des Büros des Hohen Vertreters, die Abhaltung von Wahlen (Oktober 2010) und die EU-Visaliberalisierung. In seinen Wortmeldungen betonte Österreich die Unterstützung für den Hohen Vertreter, die Notwendigkeit der Durchführung politischer Reformen (u.a. Anpassung der Verfassung an europäische Standards), verurteilte die nationalistische Rhetorik der Republika Srpska, begrüßte die Bemühungen zur regionalen Versöhnung und unterstrich das bestehende Bekenntnis der EU zu einer europäischen Perspektive für Bosnien und Herzegowina. 


\section{Zypern}

Die im September 2008 begonnenen Direktverhandlungen zwischen den beiden Volksgruppenführern Dimitris Christofias und Mehmet Ali Talat bzw. (ab Mai 2010) Dervis Eroğlu über eine Wiedervereinigung der Insel wurden fortgeführt. Der SRSG für Gute Dienste in Zypern, Alexander Downer, der SRSG für Zypern, Tayé-Brook Zerihoun bzw. seine Nachfolgerin Lisa Buttenheim und der ASG Atul Khare berichteten dem VN-SR, auf Basis der VN-GS Berichte zur Friedenstruppe der VN in Zypern (UNFICYP) und zu den Guten Diensten, insgesamt sechs Mal über die Fortschritte in den Verhandlungen und die Lage in Zypern, die als stabil und ruhig beschrieben wurde. Nach dem Treffen der beiden Volksgruppenführer mit dem VN-GS am 18. November 2010 in New York präsentierte SRSG Downer dem VN-SR Ende November 2010 den ausführlichen Fortschrittsbericht des VN-GS zu den Verhandlungen und drückte Besorgnis über den Mangel an Fortschritten aus. Der VN-SR unterstützte die laufenden Direktverhandlungen und unterstrich die Bedeutung vertrauensbildender Maßnahmen für die Erzielung weiterer Fortschritte; zahlreiche Mitglieder des VN-SR äußersten jedoch Ende 2010 Sorge über mangelnde Fortschritte. Das Mandat von UNFICYP wurde viermal unverändert um jeweils sechs Monate verlängert, zuletzt mit SR-Resolution 1953 (2010) bis 15. Juni 2011. Die Türkei hielt fest, dass sie der Präsenz von UNFICYP auf der Insel prinzipiell positiv gegenüberstehe, stimmte aber in allen Abstimmungen mit dem Argument, dass die VN seit Bestehen der Mission immer nur die Zustimmung der Republik Zypern - und nicht jene der türkisch-zypriotischen Seite - eingeholt habe, als einziges SR-Mitglied gegen die jeweilige Resolution.

Österreich drückte in diesen Debatten seine Unterstützung für UNFICYP und die Guten Dienste des VN-GS sowie für die Direktverhandlungen aus. Weiters unterstrich Österreich, dass jegliche Vereinbarung die Unterstützung der Mehrheit beider Volksgruppen genießen müsse und betonte, dass der Verhandlungsprozess in zypriotischen Händen sei. Österreich rief jedoch gleichzeitig die Parteien zur Intensivierung ihrer Bemühungen und zur Umsetzung vertrauensbildender Maßnahmen auf und hob die Vorteile einer Lösung für beide Seiten hervor.

\section{Georgien}

Nachdem im Februar 2009 mit Annahme der SR-Resolution 1866 (2009) noch eine Verlängerung des Mandats der VN-Beobachtermission in Georgien (UNOMIG) bis Mitte Juni 2009 gelungen war, scheiterte am 15. Juni 2009 der Versuch, das Mandat und damit die Aufgaben von UNOMIG an die veränderten Gegebenheiten nach dem Konflikt zwischen der Russischen Föderation und Georgien im August 2008 anzupassen. Nachdem Österreich - nach einigem Widerstand - im Juni 2009 auch in die Arbeiten der westlichen Mitglieder der „Group of Friends of Georgia“ einbezogen wurde, wurde es gemein- 


\section{Lateinamerika}

sam mit Kroatien, Frankreich, Deutschland, Türkei, Großbritannien und den Vereinigten Staaten von Amerika zu einem Miteinbringer eines Resolutionsentwurfs, der eine technische Verlängerung des Mandats von wenigen Tagen vorgesehen hätte, um weiter über die Zukunft von UNOMIG verhandeln zu können. Die Annahme dieses Resolutionsentwurfs scheiterte an einem Veto der Russischen Föderation. Es war dies das einzige Veto während österreichischer Mitgliedschaft im VN-SR. Nach der Anerkennung der Unabhängigkeit von Abchasien und Südossetien durch die Russische Föderation wollte diese eine weitere (kurzfristige) Verlängerung der Mission in der bestehenden Form nicht mehr annehmen. Für die Unterstützer der Resolution war hingegen ein Text ohne Verweis auf SR-Resolution 1808 (2008), in welcher der VN-SR die territoriale Unversehrtheit Georgiens bekräftigt hatte, nicht akzeptabel. Die 1993 eingerichtete VN-Mission wurde somit am 16. Juni 2009 eingestellt. In Votumserklärungen äußerten zahlreiche SR-Mitglieder, so auch Osterreich, ihr Bedauern darüber, dass im VN-SR keine Einigung möglich gewesen sei. Im VN-SR kam es in Folge zu keiner weiteren Behandlung der Situation in Georgien. Um die Rolle der VN als Ko-Vorsitz im Rahmen der Genfer Gespräche über Sicherheit und Stabilität und die Rückkehr von Flüchtlingen und Binnenvertriebenen sowie in den regelmäßigen Treffen des sog. „Incident Prevention und Response Mechanism“ weiterhin wahrnehmen zu können, richtete der VN-GS ein Liaisonarrangement, unter der Leitung erst von SRSG Johan Verbeke, dann von Antti Turunen, mit Sitz in Genf ein, das im November 2009 operativ wurde. 2010 war der VN-SR nicht aktiv mit der Situation in Georgien befasst.

\section{e) Lateinamerika}

\section{Haiti}

Von 11.-14. März 2009 unternahm der VN-SR eine Reise nach Haiti, um sich ein Bild über die Umsetzung des Mandats der Stabilisierungsmission der VN in Haiti (MINUSTAH) zu machen. Aus den damaligen Gesprächen mit Regierungsmitgliedern, politischen Vertretern, der Zivilgesellschaft und dem Führungspersonal der VN-Mission ging hervor, dass die politische Lage in Haiti - insbesondere auch wegen der Präsenz von MINUSTAH - in den vergangenen Jahren stabilisiert werden konnte.

Der VN-SR befasste sich in den Jahren 2009/10 auf der Grundlage halbjährlicher Berichte des VN-GS sowie anlässlich aktueller Entwicklungen wie des Erdbebens vom Jänner 2010 und der Wahlen im November 2010 eingehend mit der Lage in Haiti. Im Rahmen der offenen Debatte am 6. April 2009 berichtete der damalige SRSG, Hédi Annabi, über die Fortschritte bei der Stabilisierung Haitis, unterstrich jedoch die dringende Notwendigkeit einer Verbesserung der Sicherheitslage bei gleichzeitiger wirtschaftlicher und sozialer Entwicklung. Österreich hob die Notwendigkeit einer weiteren Stär- 
kung der Rechtsstaatlichkeit hervor, u.a. durch eine Reform der Jugendgerichtsbarkeit, die eine Voraussetzung für die Schaffung eines umfassenden Systems zum Schutz von Kindern darstellt. In seiner Vorsitzerklärung (S/PRST/2009/4) erkannte der VN-SR die Bedeutung der Abhaltung der internationalen Geberkonferenz für Haiti am 14. April 2009 in Washington an und rief die haitianischen Institutionen zur verstärkten Zusammenarbeit bei der Förderung des Dialogs, der Rechtsstaatlichkeit und der guten Regierungsführung auf. Am 19. Mai 2009 ernannte der VN-GS den früheren USPräsidenten Bill Clinton zu seinem Sondergesandten (SE) für Haiti, der sich seither auf den wirtschaftlichen Wiederaufbau des Landes konzentriert. Mit SR-Resolution 1892 (2009) wurde das Mandat von MINUSTAH um weitere zwölf Monate verlängert. Österreich erwirkte gemeinsam mit Costa Rica die Aufnahme mehrerer Referenzen auf den Schutz und die Rechte von Kindern in den Resolutionstext und setzte sich für einen Verweis auf die Stärkung der Rolle von Frauen im politischen Prozess in Haiti ein.

In Folge des schweren Erdbebens in Haiti am 12. Jänner 2010, bei dem auch die Führung von MINUSTAH (SRSG Annabi) sowie zahlreiche VN-Bedienstete und Militär- und Polizeipersonal ums Lebens kamen, nahm der VN-SR am 19. Jänner SR-Resolution 1908 (2010) an, mit der die Truppenstärke der Mission temporär um 1.500 Personen in der Polizei- und 2.000 Personen in der Militärkomponente erhöht wurde. Dadurch sollte MINUSTAH in die Lage versetzt werden, die unmittelbaren Hilfsmaßnahmen sowie die Bemühungen zum Wiederaufbau zu unterstützen und Stabilität in Haiti zu gewährleisten. In einer Presseerklärung (SC/9846) vom 18. Jänner 2010 bekundete der VN-SR seine tiefe Anteilnahme gegenüber der Regierung und Bevölkerung von Haiti angesichts der erlittenen Verluste und sprach den Angehörigen der verstorbenen VN-MitarbeiterInnen sein Beileid aus.

Am 28. April 2010 schlug SRSG Edmond Mulet, der dem beim Erdbeben verstorbenen Hédi Annabi als Leiter von MINUSTAH nachfolgte, dem VN-SR vor, dass MINUSTAH neben der Übernahme der Leitungsrolle bei der Unterstützung der geplanten Wahlen vermehrt zur Förderung der Rechtsstaatlichkeit beitragen sollte. Zur Gewährleistung von Sicherheit und Stabilität im Hinblick auf die Wahlen und eines wirksamen Schutzes der Zivilbevölkerung wurde darüber hinaus vorgeschlagen, die Polizeikomponente von MINUSTAH um weitere 680 Personen anzuheben. Österreich betonte, dass die VN weiterhin die Schlüsselrolle bei der Koordination der internationalen Hilfe in Kooperation mit den haitianischen Behörden innehaben sollte und nannte die Stärkung der Rechtsstaatlichkeit, den Kampf gegen die Straflosigkeit, gute Regierungsführung und den Schutz von Zivilisten, insbesondere von Frauen und Kindern vor sexueller Gewalt, als Prioritäten für die Arbeit von MINUSTAH in den kommenden Monaten. Mit SR-Resolution 1927 (2010) vom 4. Juni 2010 beschloss der VN-SR die temporäre Aufstockung der Polizeikomponente von MINUSTAH sowie die Anpassung des Mandats, die eine logistische Unterstützung und Bereitstellung technischer 


\section{Lateinamerika}

Expertise durch MINUSTAH insbesondere im Bereich der Rechtsstaatlichkeit vorsah.

In der offenen Debatte am 13. September 2010 berichtete SRSG Mulet, dass die geplante Abhaltung der Wahlen im November eine wichtige Etappe im Prozess der demokratischen Konsolidierung darstelle. Als beunruhigend erweise sich die Lage in den Flüchtlingslagern, wo besonders Frauen und Kinder dem Risiko von sexueller Gewalt ausgesetzt seien. Österreich bekräftigte die Bedeutung der Schaffung eines soliden Schutzumfelds in Haiti und äußerte die Hoffnung, dass die Bedürfnisse der vertriebenen Kinder und der Kampf gegen den Kinderhandel weiterhin Schwerpunkte von MINUSTAH darstellen würden. Mit SR-Resolution 1944 (2010) verlängerte der VN-SR das Mandat von MINUSTAH um weitere zwölf Monate bis zum 15. Oktober 2011. Österreich konnte erreichen, dass der VN-GS darin zur Berichterstattung über den Schutz der Zivilbevölkerung aufgefordert wird. Weiters wurden auf österreichischen Vorschlag Ergänzungen zur Förderung der Beteiligung von Frauen an den Wahlen bzw. zur Berücksichtigung der besonderen Bedürfnisse von Frauen und Kindern in Haiti in die Resolution aufgenommen. Nach Abhaltung der ersten Runde der Präsidentschaftswahlen und der Wahlen zum Parlament am 28. November 2010 informierten ASG Khare sowie der UGS für Peacekeeping Alain Le Roy den VN-SR Ende November bzw. am 10. Dezember 2010 über die Lage in Haiti. In einer Presseerklärung (SC/10110) äußerte sich der VN-SR besorgt hinsichtlich der Vorwürfe des Wahlbetrugs und rief die KandidatInnen auf, Ruhe zu bewahren und eine Lösung im Rahmen der vorgesehenen rechtlichen Mechanismen zu suchen.

\section{Sonstige Konflikte}

\subsection{Honduras}

Die Entmachtung von Präsident José Manuel Zelaya durch Militär und Justiz am 28. Juni 2009 führte zu einer schweren innenpolitischen Krise in Honduras. Am 25. September 2009 informierte der brasilianische Außenminister Celso Amorim den VN-SR über die Lage in Honduras nach der Rückkehr des gestürzten Präsidenten Zelaya nach Tegucigalpa, wo dieser in der brasilianischen Botschaft Aufnahme gefunden hatte. In den anschließenden geschlossenen Konsultationen wurde die Unerlässlichkeit einer regionalen Lösung der Krise basierend auf dem Abkommen von San José betont. Österreich drückte seine volle Unterstützung für die regionalen Bemühungen zur Beilegung der Krise und seine Besorgnis über Menschenrechtsverletzungen aus. Der VN-SR einigte sich auf Bemerkungen des SR-Vorsitzes gegenüber der Presse, in denen die Wichtigkeit des Respekts für die Unversehrtheit der brasilianischen Botschaft und der sich darin befindlichen Personen unterstrichen und alle Parteien zu einer friedlichen Konfliktlösung aufgefordert werden. Der VN-SR war danach nicht mehr mit der Situation in Honduras befasst. 


\subsection{Venezuela und Kolumbien}

Im November 2009 lösten die kurz davor erfolgte Unterzeichnung eines Militärabkommens zwischen den Vereinigten Staaten von Amerika und Kolumbien sowie Grenzzwischenfälle und anschließende verbale Kriegsdrohungen seitens Präsident Hugo Chávez bilaterale Spannungen zwischen Venezuela und Kolumbien aus. Im Rahmen der offenen Debatte des VN-SR zum Schutz der Zivilbevölkerung in bewaffneten Konflikten am 11. November 2009 sowie des öffentlichen Briefings der Vorsitzenden der AntiterrorismusKomitees am 13. November 2009 thematisierten Venezuela, Kolumbien und die Vereinigten Staaten von Amerika den bilateralen Konflikt. Die Ständigen Vertreter Kolumbiens und Venezuelas bei den VN hatten sich zuvor mit dem Ersuchen um Befassung des VN-SR an Botschafter Thomas Mayr-Harting in dessen Eigenschaft als Vorsitzender des VN-SR gewandt. Österreich setzte sich hinter den Kulissen in enger Abstimmung mit den Vereinigten Staaten von Amerika und den lateinamerikanischen Mitgliedern des VN-SR, Mexiko und Costa Rica, für eine Lösung des Konflikts im regionalen Rahmen ein und konnte (zur Zufriedenheit beider Seiten) zu einer Beruhigung der Situation beitragen. $\mathrm{Zu}$ einer formellen Behandlung im VN-SR kam es nicht, womit nach allgemeiner Ansicht eine weitere Eskalation der Situation vermieden werden konnte.

\subsection{Costa Rica und Nicaragua}

Anlässlich eines Grenzkonflikts zwischen Nicaragua und Costa Rica im November 2010 informierte der Ständige Vertreter Costa Ricas bei den VN den britischen Vorsitzenden des VN-SR über die aus Sicht Costa Ricas vorliegende Verletzung der Souveränität Costa Ricas. Nicaragua habe insbesondere eine militärische Präsenz auf der zum Hoheitsgebiet von Costa Rica gehörenden Insel Calero etabliert. Costa Rica erwäge daher auch, den VN-SR mit der Angelegenheit zu befassen, sollte der Konflikt nicht auf bilateraler oder regionaler Ebene gelöst werden können. Österreich führte in New York sowohl mit Nicaragua als auch mit Costa Rica Gespräche und plädierte für eine Lösung auf regionaler Ebene unter Vermittlung der Organisation Amerikanischer Staaten (OAS). Der VN-SR wurde bis Ende 2010 nicht mit der Situation befasst. 


\section{Die Tätigkeit des Sicherheitsrats und die österreichische Mitwirkung - Querschnittsthemen \\ 1. Schutz der Zivilbevölkerung in bewaffneten Konflikten}

Der zu den österreichischen Prioritäten im VN-SR zählende Schutz der Zivilbevölkerung, insbesondere von Frauen und Kindern (nähere Ausführungen siehe Kapitel III.2, Kapitel III.3), in bewaffneten Konflikten war ein Schwerpunkt des österreichischen Vorsitzes im VN-SR im November 2009. Am 11. November 2009 nahm der VN-SR unter dem Vorsitz von Bundesminister Michael Spindelegger einstimmig SR-Resolution 1894 (2009) zum Schutz von Zivilisten in bewaffneten Konflikten an, welche von insgesamt $31 \mathrm{VN}$ Mitgliedstaaten miteingebracht wurde. Die Resolution wurde von Österreich vorbereitet und mit den übrigen SR-Mitgliedern verhandelt (siehe auch Kapitel IV).

SR-Resolution 1894 (2009) zielt darauf ab, die Einhaltung der internationalen Standards (humanitäres Völkerrecht, Menschenrechte, Flüchtlingsrecht) zum Schutz der Zivilbevölkerung durch Konfliktparteien zu verbessern. Der VN-SR bekräftigt darin auch seine Bereitschaft, bei vorsätzlichen Angriffen auf Zivilpersonen oder der gezielten Behinderung von humanitären Hilfsmaßnahmen erforderlichenfalls geeignete Maßnahmen zu ergreifen, die ihm nach der Satzung der VN zur Verfügung stehen. Weiters spricht sich der VN-SR mit klaren Worten gegen die Straflosigkeit von schweren Übergriffen auf die Zivilbevölkerung aus und führt erstmals in einer SR-Resolution umfassend die verschiedenen Mechanismen strafrechtlicher Verantwortlichkeit (strafrechtliche Verfolgung, Reparationszahlungen an die Opfer, Errichtung von Wahrheitskommissionen und Institutionenreform) an. Einen zentralen Teil der SR-Resolution 1894 (2009) bilden konkrete Maßnahmen, die eine wirksamere Umsetzung von Schutzaufgaben durch FEO der VN gewährleisten sollen (etwa die Erarbeitung eines Operationskonzepts für FEO zum Schutz der Zivilbevölkerung, die Entwicklung missionsspezifischer Schutzstrategien durch FEO oder ein verbessertes Training für Blauhelme). Mit SRResolution 1894 (2009) wird die internationale Gemeinschaft zudem aufgefordert, Hilfe bei der Betreuung und Rehabilitierung sowie bei der wirtschaftlichen und sozialen Wiedereingliederung der Opfer von Minen bereitzustellen. Gleichzeitig wird der Opferhilfe-Bereich, ein Schwerpunkt der Streumunitions- und Landminenproblematik, mit SR-Resolution 1894 (2009) erstmals umfassend im VN-Rahmen verankert und anerkannt. An der Debatte am 11. November 2009 beteiligten sich neben VN-GS Ban Ki-moon und UGS Holmes auch - über österreichische Initiative - die stellvertretende VNHochkommissarin für Menschenrechte Kyung-wha Kang, womit zum ersten Mal seit 2005 ein Auftritt der Hochkommissarin bzw. ihrer Stellvertreterin im VN-SR gegen einige Widerstände sichergestellt werden konnte. Weiters nahmen insgesamt 63 VN-Mitgliedstaaten an der Debatte teil. 
Im Jahr 2010 fokussierten sich die österreichischen Bemühungen zum Schutz der Zivilbevölkerung auf eine zügige Umsetzung der in SR-Resolution 1894 (2009) vorgesehenen Maßnahmen. Dazu zählten etwa die Erarbeitung einer operativen Anleitung für FEO zur Umsetzung von Schutzmandaten, die Erarbeitung eines Rahmenwerks für missionsspezifische Schutzstrategien, die Entwicklung von Trainingsmodulen sowie Bemühungen zur verbesserten Berichterstattung. Die offene Debatte am 7. Juli 2010 diente einer ersten Bilanzierung der bisher erzielten Fortschritte. Am 22. November 2010 behandelte der VN-SR in einer weiteren offenen Debatte den jüngsten Bericht des VN-GS zum Schutz der Zivilbevölkerung in bewaffneten Konflikten (S/2010/579), der schwerpunktmäßig der Umsetzung und Weiterentwicklung von SR-Resolution 1894 (2009) gewidmet ist. Im Rahmen der Debatte nahm der VN-SR eine Vorsitzerklärung (S/PRST/2010/25) an, mit der die unter österreichischem Vorsitz begonnenen Arbeiten zur Verbesserung des Schutzes von Zivilisten in bewaffneten Konflikten eine Fortsetzung fanden. Auf österreichische Initiative betont diese Vorsitzerklärung die Bedeutung nachhaltiger Lösungen für Flüchtlinge und Binnenvertriebene, sowie (u.a. in Reaktion auf den von der Regierung des Tschad geforderten Abzug von MINURCAT) von „Benchmarks“ im Zusammenhang mit der Beendigung von FEO der VN. Im Lichte der Massenvergewaltigungen im Sommer $2010 \mathrm{im}$ Osten der Demokratischen Republik Kongo setzte sich Österreich weiters für die Aufnahme eines Absatzes zur Notwendigkeit der Kommunikation von FEO mit der lokalen Bevölkerung ein. Österreich bemühte sich außerdem erfolgreich um die Annahme eines revidierten Aide Mémoire des VN-SR zum Schutz von Zivilisten in bewaffneten Konflikten im Annex der Vorsitzerklärung. Es handelt sich hierbei um ein praktisches Instrument des VN-SR, das diesem die systematische Analyse von und kohärente Reaktion auf Probleme rund um den Schutz der Zivilbevölkerung erleichtern soll. Die Entwicklungen seit der Annahme der letzten Version des Aide Mémoire mit der Vorsitzerklärung vom 14. Jänner 2009 (S/PRST/2009/1) und die durch SRResolution 1894 (2009) erzielten Fortschritte hatten eine Überarbeitung dieses Dokuments erforderlich gemacht.

Österreich setzte sich auch im Rahmen der Verhandlungen des VN-SR zur Verlängerung der Mandate von FEO der VN zum Schutz der Zivilbevölkerung konsequent dafür ein, die Bestimmungen von SR-Resolution 1894 (2009) in diese Mandate einfließen zu lassen. Dies ist u.a. bei den VN-Missionen in Afghanistan, in der Demokratischen Republik Kongo, im Sudan und in Côte d'Ivoire gelungen. Im Falle von MINURCAT, die als Mission zum Schutz der Zivilbevölkerung in den Flüchtlingslagern im Osten des Tschad und der Zentralafrikanischen Republik konzipiert war, zeigte sich Österreich über den von Präsident Déby Anfang 2010 geforderten Abzug der Mission sehr besorgt, zumal ein funktionierender Schutz der Zivilbevölkerung alles andere als gewährleistet erschien. Österreich konnte sich in den schwierigen Verhandlungen mit der Forderung durchsetzen, dass in SR-Resolution 1923 (2010) zur Verlängerung von MINURCAT bis Ende 2010 mit einem reduzier- 
ten Mandat - welches nur mehr den Schutz der zivilen VN-Präsenz, nicht jedoch der Zivilbevölkerung vorsah - eine Notfallsklausel aufgenommen wurde, die MINURCAT autorisierte, auf Akte der Gewalt gegen Zivilisten im unmittelbaren Umfeld der Mission zu reagieren.

Außerdem hat sich Österreich über die gesamte Dauer seiner Mitgliedschaft im VN-SR aktiv an den Sitzungen der Informellen Expertengruppe des VN-SR zum Schutz der Zivilbevölkerung, die im Vorfeld der Verlängerung der Mandate von FEO der VN oder der Autorisierung von VN-mandatierten Operationen stattgefunden haben, beteiligt.

In Fortsetzung seines Engagements im VN-SR hat Österreich auch auf eine Stärkung des Schutzes der Zivilbevölkerung in den militärischen Operationen und zivilen Missionen der EU hingewirkt und eine Überarbeitung der diesbezüglichen EU-Leitlinien erzielt.

\section{Frauen, Frieden und Sicherheit - Bekämpfung sexueller Gewalt in Konflikten}

$\mathrm{Zu}$ den Prioritäten Österreichs im VN-SR zählte weiters - in konsequenter Fortsetzung der bestehenden außenpolitischen Prioritäten und in engem Zusammenhang mit dem Schwerpunkt zum Schutz der Zivilbevölkerung der Bereich Frauen, Friede und Sicherheit. Die 2009 angenommenen SRResolutionen 1888 (2009) und 1889 (2009) zielen auf eine Stärkung der Maßnahmen zur Bekämpfung von sexueller Gewalt gegen Frauen in bewaffneten Konflikten sowie auf die verbesserte Berücksichtigung der Prioritäten von Frauen und Mädchen in Postkonfliktsituationen und im Bereich des Peacebuilding ab. SR-Resolution 1888 (2009) sieht u.a. die Ernennung einer/eines SRSG zu sexueller Gewalt in Konflikten sowie die stärkere Berücksichtigung von sexueller Gewalt bei der Verhängung gezielter Sanktionen durch den VN-SR vor, und beschließt die Einrichtung eines Expertenteams, um nationale Behörden in Konfliktländern mit vorherrschender sexueller Gewalt im Bereich der Rechtsstaatlichkeit zu unterstützen. Österreich setzte sich in den Verhandlungen zu beiden Resolutionen und in den offenen Debatten des VN-SR (insgesamt drei im Jahr 2009) für ein Ende der Straflosigkeit von Tätern sexueller Gewalt, stärkere Verantwortlichkeitsmechanismen, eine systematischere Berichterstattung an den VN-SR, eine stärkere Einbindung von Frauen in Friedensverhandlungen und Wiederaufbauprozesse sowie eine verstärkte Ernennung von Frauen für Senior-Management Positionen in den VN sowie für FEO der VN ein. Beide Resolutionen wurden von Österreich miteingebracht.

Das Jahr 2010 stand im Zeichen des 10-jährigen Jubiläums der Annahme von SR-Resolution 1325 (2000) zu Frauen, Frieden und Sicherheit. Im April 2010 erfolgte die erste Unterrichtung des VN-SR durch die im Februar 2010 bestellte Margot Wallström zur SRSG zu sexueller Gewalt in Konflikten. 
Unter dem Vorsitz Ugandas fand aus Anlass des 10-jährigen Jubiläums am 26. Oktober 2010 eine offene Debatte unter Teilnahme von Bundesminister Michael Spindelegger statt, zu der eine Vorsitzerklärung des VN-SR (S/PRST/2010/22) angenommen wurde. Die Debatte sowie die Vorbereitungen dazu stießen auf großes Interesse der VN-Mitgliedstaaten sowie der Zivilgesellschaft. Mehrere SR-Mitglieder, so auch Österreich, kündigten im Rahmen dieser Debatte konkrete nationale Verpflichtungen zur verbesserten Umsetzung von SR-Resolution 1325 (2000) an.

Österreich war intensiv an den Vorbereitungen zum 10. Jahrestag und u.a. an den Verhandlungen über eine Vorsitzerklärung (S/PRST/2010/22) beteiligt, mit welcher der VN-SR eine Reihe von Indikatoren verabschiedete, die Fortschritte in der Umsetzung von SR-Resolution 1325 (2000) besser nachvollziehbar und quantifizierbar machen werden (Österreich hatte die Entwicklung dieser Indikatoren durch die Veranstaltung eines Seminars im März 2010 in New York unterstützt). Mithilfe dieser Indikatoren sollen künftig u.a. Daten über das Ausmaß sexueller Gewalt an Frauen in Konfliktgebieten sowie über die dagegen ergriffenen Maßnahmen systematisch gesammelt werden. Laufend soll weiters auch der Anteil von Frauen an leitenden Funktionen in FEO der VN sowie im politischen Leben, in den Sicherheitsstrukturen und im Justizapparat der VN-Mitgliedstaaten erfasst werden. In Vorbereitung der offenen Debatte veranstaltete Österreich gemeinsam mit Mexiko und Großbritannien ein Treffen des VN-SR im Arria-Format zur Frage einer verbesserten Umsetzung der Resolution in von bewaffneten Konflikten betroffenen Gebieten, und ermöglichte so den VertreterInnen der Zivilgesellschaft, ihre Positionen und Vorschläge in die Arbeiten des VN-SR einzubringen.

Ein wesentlicher Schritt zur Bekämpfung sexueller Gewalt in bewaffneten Konflikten gelang im Dezember 2010. Unter dem Vorsitz der Vereinigten Staaten von Amerika beschloss der VN-SR die Einrichtung von „Monitoring, Analysis and Reporting“ Arrangements ähnlich dem bereits bestehenden System zum Schutz von Kindern in bewaffneten Konflikten, mit Annahme der SR-Resolution 1960 (2010). Mit dieser wird der VN-GS beauftragt, in seinen jährlichen Berichten an den VN-SR zu sexueller Gewalt in bewaffneten Konflikten jene Konfliktparteien aufzulisten, die Vergewaltigungen und andere Formen sexueller Gewalt (in Konfliktsituationen, die sich auf der Tagesordnung des VN-SR befinden) begehen oder dafür verantwortlich sind. Von diesen Parteien eingegangene konkrete Verpflichtungen (deren Umsetzung Voraussetzung für eine Streichung von dieser Liste des VN-GS ist) sollen systematisch durch den VN-GS überwacht und an den VN-SR berichtet werden. Österreich hatte sich in den Resolutionsverhandlungen nachdrücklich für diese Resolutionselemente eingesetzt, mit denen wichtige Lücken in der Umsetzung der SR-Resolutionen 1820 (2008) und 1888 (2009) geschlossen werden. Auch SR-Resolution 1960 (2010) wurde von Österreich miteingebracht. 
Darüber hinaus ließ Österreich die Zielsetzungen der SR-Resolutionen 1325 (2000) und 1820 (2008) sowie deren Nachfolgeresolutionen in die tägliche (insbesondere länderspezifische) Arbeit des VN-SR einfließen und trat bei der Verlängerung von bestehenden Mandaten von VN-Friedensmissionen erfolgreich für eine stärkere Berücksichtigung von Frauenanliegen ein (z.B. Afghanistan, Timor-Leste, Haiti, Demokratische Republik Kongo).

\section{Kinder und bewaffnete Konflikte}

Auch im bestehenden System zum Schutz von Kindern in bewaffneten Konflikten konnten 2009/2010 beträchtliche Fortschritte erzielt werden. Im Mittelpunkt der jährlichen Debatte im April 2009 (Vorsitz Mexiko) stand der Vorschlag des VN-GS, nicht nur jene Konfliktparteien in die „schwarzen Listen“ des VN-GS aufzunehmen, die sich der Rekrutierung von Kindersoldaten schuldig machen, sondern auch solche, die im Kontext eines bewaffneten Konflikts Kinder töten, verstümmeln oder sexuelle Gewalt gegen Kinder verüben. Die Aufnahme von Konfliktparteien in diese schwarzen Listen führt zur Auslösung eines Überwachungs- und Berichtsmechanismus der VN. Mangels Einigung unter den SR-Mitgliedern konnte diese von Österreich unterstützte Forderung jedoch im April 2009 noch nicht beschlossen werden. Österreich setzte sich in weiterer Folge aktiv für die Ausweitung der Kriterien auf die Tatbestände der Tötung und Verstümmelung sowie der sexuellen Gewalt gegen Kinder im Wege einer Resolution ein. Diese gelang am 4. August 2009 mit der einstimmigen Annahme der SR-Resolution 1882 (2009). Österreich konnte weiters auch Vorschläge zur Verbesserung des Informationsflusses zwischen der Arbeitsgruppe des VN-SR zu Kindern und bewaffneten Konflikten und den VN-SR Sanktionenkomitees sowie zur Stärkung des Kampfes gegen die Straflosigkeit in der Resolution verankern.

Zu den Hauptthemen der offene Debatte im Juni 2010 zählte die Forderung nach verstärkten Maßnahmen gegen jene Konfliktparteien, die in fortgesetzter oder besonders gravierender Weise Rechtsverletzungen gegen Kinder in Konfliktsituationen begehen (sog. ,persistent perpetrators“). In der als Ergebnis der Debatte angenommenen Vorsitzerklärung (S/PRST/2010/10) bringt der VN-SR die Bereitschaft zum Ausdruck, Sanktionen gegen diese „persistent perpetrators“ zu verhängen, und sieht - auf Basis eines österreichischfranzösischen Vorschlags - weiters detaillierte Bestimmungen vor, um dies in der Praxis zu ermöglichen (u.a. verstärkter Informationsaustausch der SRSG für Kinder und bewaffneten Konflikte sowie der Arbeitsgruppe zu Kindern und bewaffneten Konflikten mit den VN-SR Sanktionenkomitees). Österreich konnte sich im Zuge der Verhandlungen dieser Vorsitzerklärung weiters erfolgreich für die Verankerung eines Länderbesuches durch die Arbeitsgruppe innerhalb eines Jahres einsetzen. In der Debatte unterstrich Österreich die vom VN-GS betonte Notwendigkeit direkter Kontakte der VN mit nichtstaatlichen Konfliktparteien zur Ausarbeitung von Aktionsplänen, 
rief die Konfliktparteien auf, sämtliche Kinderrechtsverletzungen einzustellen und drückte weiters Besorgnis über die zunehmenden Angriffe auf Schulen aus.

Die Arbeitsgruppe des VN-SR zum Thema Kinder und bewaffnete Konflikte unter dem Vorsitz Mexikos hat zu insgesamt elf Ländersituationen (Afghanistan, Burundi, Demokratische Republik Kongo, Myanmar, Sudan, Zentralafrikanische Republik, Uganda, Sri Lanka, Kolumbien, Philippinen, Nepal) unter aktiver österreichischer Beteiligung Schlussfolgerungen ausgearbeitet, die auf Grundlage der länderspezifischen Berichte des VN-GS konkrete Empfehlungen und Aufforderungen an die jeweiligen Konfliktparteien zur Beendigung von Verbrechen gegen Kinder, zur Bekämpfung der Straflosigkeit sowie zur Ausarbeitung konkreter Aktionspläne zur Entlassung von Kindersoldaten enthalten. Weiters absolvierte die Arbeitsgruppe im November 2010 unter österreichischer Beteiligung den ersten Länderbesuch (wie in PRST/2010/10 vorgesehen) und zwar in Nepal. Ziel dieses Besuchs war die Überprüfung der Umsetzung des von den Konfliktparteien unterzeichneten Aktionsplans und die Identifizierung der noch bestehenden Hürden für die Reintegration ehemaliger Kindersoldaten.

\section{Peacekeeping}

Friedenserhaltende Operationen (FEO) der VN leisten seit 1948 einen wichtigen und anerkannten Beitrag zur Wahrung des Weltfriedens und der internationalen Sicherheit. Der VN-SR beschließt die Mandate für FEO und trägt die politische Hauptverantwortung für den weltweiten Einsatz von VN-Friedenssicherungskräften. Bei der Durchführung der Operationen kommt dem VN-Sekretariat und den Truppenstellern eine Schlüsselrolle zu. Als SR-Mitglied war Österreich in sämtliche Beratungen des VN-SR über die Verlängerung bzw. Beendigung von FEO eingebunden. Aufgrund seiner langjährigen Erfahrungen als Truppensteller konnte Österreich gestaltend an dieser Kernaufgabe des VN-SR mitwirken und sich mit eigenen Schwerpunkten in die Mandatsverhandlungen einbringen.

Während der österreichischen SR-Mitgliedschaft hat sich die Gesamtzahl von FEO mit 16 Operationen zum Jahresende 2010 gegenüber 17 zu Beginn des Jahres 2009 geringfügig verändert. Das Mandat von MINURCAT wurde nach dem Auslaufen der EU-Überbrückungsoperation EUFOR Tchad/RCA im März 2009 um eine militärische Komponente erweitert und ist mit 31. Dezember 2010 ausgelaufen. UNOMIG wurde aufgrund des Vetos der Russischen Föderation gegen eine neuerliche Verlängerung mit Juni 2009 beendet. UNMIK wurde nach der Übergabe aller Aufgaben an die neu geschaffene EU-Rechtsstaatlichkeitsmission EULEX Kosovo auf ein Minimum verkleinert. Die Militär- und Polizeikomponenten von MINUSTAH mussten aufgrund des Erdbebens in Haiti am 12. Jänner 2010 signifikant aufgestockt werden. Die Operation in der Demokratischen Republik Kongo 
(MONUC) wurde in eine Stabilisierungsmission (MONUSCO) umgewandelt und in ihrer Personalstärke reduziert.

Mit einem Höchststand von rund 125.000 entsandten Truppen, PolizistInnen und zivilen ExpertInnen im Jahr 2010 hat sich die Zahl von Blauhelmen seit 2000 versechsfacht, während die Strukturen für die Durchführung und Aufsicht über FEO nicht entsprechend angepasst wurden. Gleichzeitig hat sich das Aufgabenspektrum von Operationen konstant ausgeweitet. Es reicht von der Überwachung von militärischen Pufferzonen und Waffenstillständen, über die Unterstützung bei der Umsetzung von Friedensabkommen bis zur Wahrnehmung von Exekutivaufgaben und Peacebuilding-Aktivitäten wie der Stärkung der Rechtsstaatlichkeit, dem Schutz der Menschenrechte und der Sicherheitssektorreform. In Anbetracht der Zunahme innerstaatlicher Konflikte und des vermehrten Auftretens nichtstaatlicher Akteure in bewaffneten Auseinandersetzungen hat sich darüber hinaus der Schutz der Zivilbevölkerung zu einer Schlüsselaufgabe von FEO entwickelt, an der zunehmend auch deren Erfolg und die Glaubwürdigkeit der VN als Friedensstifter gemessen wird.

Vor dem Hintergrund der Begrenztheit der für FEO verfügbaren personellen und finanziellen Ressourcen sowie der komplexen und umfangreichen Anforderungen moderner multidimensionaler Friedensoperationen initiierten Großbritannien und Frankreich mit Jahresbeginn 2009 einen Reflexionsprozess zu FEO im VN-SR. Parallel dazu leitete das VN-Sekretariat im Juli 2009 mit der „New Horizon“-Initiative einen VN-weiten Reformprozess zu FEO ein. Hauptzielsetzungen der Reformbestrebungen sind die konzeptionelle Weiterentwicklung des Peacekeeping in den Bereichen Schutz der Zivilbevölkerung, Peacebuilding und robuste Friedenssicherung, Maßnahmen zur Ressourcenmobilisierung und hinsichtlich des Trainings der für FEO erforderlichen Kapazitäten, die Optimierung der logistischen Unterstützung von FEO sowie die Verbesserung der Mechanismen zur Planung und Aufsicht über FEO.

Die verstärkte Beschäftigung mit generischen Fragen von FEO im Zeitraum 2009/10 fand im VN-SR ihren Niederschlag in sechs offenen thematischen Debatten zu Peacekeeping sowie zwei geschlossenen Konsultationen. Als Ergebnis wurden zwei Vorsitzerklärungen (S/PRST/2009/24, S/PRST/2010/2) angenommen, in denen Kernpunkte der Peacekeeping-Reformagenda aufgegriffen werden. Einen zusätzlichen Impuls für den Reformprozess lieferten die unter japanischem Vorsitz abgehaltenen Beratungen der Arbeitsgruppe des VN-SR zu FEO. Schwerpunkte der Diskussionen in diesem Forum, zu denen auch Truppensteller und große Beitragszahler eingeladen wurden, waren das Auseinanderklaffen zwischen den umfangreichen Mandaten von FEO und den beschränkten Ressourcen zu deren Umsetzung im Feld, Möglichkeiten zur Stärkung der Zusammenarbeit zwischen dem VN-SR und Truppenstellern und der Kapazitätenaufbau. Der im Oktober 2010 veröffentlichte erste Fortschrittsbericht zur „New Horizon“-Initiative zieht eine Zwischenbilanz über die Fort- 
schritte bei der Umsetzung von Reformmaßnahmen und hält fest, dass nach dem Überschreiten des Personalhöchststands nunmehr eine Phase der Konsolidierung im Bereich der FEO begonnen hat. Der VN-SR hat bereits 2010 verstärkt den Übergang von FEO zu anderen Formen der VN-Präsenz und das Ineinandergreifen von Peacekeeping und Peacebuilding thematisiert.

Österreich unterstützte den Reformprozess zu FEO mit Nachdruck und brachte seine Expertise als VN-Truppensteller ein. Von Anfang an setzte sich Österreich gemeinsam mit seinen EU-Partnern im VN-SR, Frankreich und Großbritannien, für einen verbesserten Informationsaustausch zwischen VN-SR, VN-Sekretariat und Truppenstellern im Vorfeld der Mandatsverlängerungen von FEO sowie während der Durchführung von Operationen ein. Die mittlerweile fest etablierte Praxis von Truppenstellertreffen vor Mandatsverhandlungen im VN-SR ist als ein konkretes Resultat dieser Bemühungen zu sehen. Weiters trat Österreich für eine Vertiefung der Zusammenarbeit zwischen den VN und Regionalorganisationen im Krisenmanagement ein. Der Stärkung globaler Partnerschaften für die Friedenssicherung war auch ein vom BMeiA und dem BMLVS im Mai 2010 in Zusammenarbeit mit dem International Peace Institute (IPI) veranstaltetes Seminar an der Diplomatischen Akademie gewidmet, an dem VertreterInnen der VN, EU, NATO und OSZE teilnahmen. Zu guter Letzt hat Österreich die Verbesserung des Schutzes der Zivilbevölkerung durch FEO als ein zentrales Anliegen in den Reformprozess zu FEO eingebracht. Kristallisationspunkt dieses Engagements war die Annahme der SR-Resolution 1894 (2009), die weitreichende Maßnahmen für eine wirksame Umsetzung von Schutzaufgaben durch FEO vorsieht (nähere Ausführungen siehe Kapitel III.1).

Die seit 1960 anhaltende österreichische Beteiligung an FEO der VN bildet eine wesentliche Voraussetzung, um im laufenden Reformprozess als kompetenter und lösungsorientierter Partner Gehör zu finden. Seit vielen Jahren liegt Österreich im vorderen Drittel der ca. 120 Truppensteller zu FEO der VN. In den Jahren 2009/10 stellte Österreich weiterhin das größte Truppenkontingent im Rahmen von UNDOF sowie bis März 2010 mit Generalmajor Wolfgang Jilke den Truppenkommandanten. Zu UNFICYP, UNTSO, MINURSO und UNMIN entsandte Österreich kleinere Kontingente. Der letzte bei UNMIK verbliebene österreichische Polizist wurde mit Februar 2009 repatriiert. Mit der Teilnahme an der EU-Überbrückungsoperation EUFOR Tchad/RCA und der bis Ende 2009 befristeten Beteiligung an der VNMission MINURCAT konnte Österreich zudem sein Engagement für den Schutz von Zivilpersonen und die Sicherstellung humanitärer Hilfe in einer Krisenregion konkret unter Beweis stellen.

\section{Peacebuilding}

In Ergänzung der Debatten zu Peacekeeping widmete der VN-SR 2009/2010 drei Debatten dem Thema „Post-Conflict Peacebuilding“ auf Grundlage der 


\section{Peacebuilding}

beiden Berichte des VN-GS zu Peacebuilding in unmittelbaren Post-Konfliktsituationen (S/2009/304, S/2010/386), wobei jeweils Vorsitzerklärungen dazu angenommen wurden (S/PRST/2009/23, S/PRST/2010/7, S/PRST/2010/20). $\mathrm{Zu}$ den Hauptthemen zählten die Bedeutung des nationalen „Ownership“ sowie des nationalen Kapazitätenaufbaus als Grundlage für Peacebuilding, das Erfordernis der zeitlichen Parallelität von Peacekeeping und Peacebuilding-Aufgaben, die Zusammenarbeit der $\mathrm{VN}$ mit anderen Internationalen Organisationen und Regionalorganisationen sowie die Frage des Übergangs von FEO zu sog. „Integrated Peacebuilding Offices“, wie sie derzeit etwa in Guinea-Bissau oder Sierra Leone bestehen. Österreich betonte in diesen Debatten u.a. seine Unterstützung für die Arbeit der PBC (Österreich wurde im Juli 2009 in die länderspezifische Konfiguration der PBC zu Sierra Leone aufgenommen) und forderte eine verstärkte Einbindung der PBC in die Arbeit des VN-SR, inklusive in der Erarbeitung von Mandaten für FEO der VN. Weiters setzte sich Österreich für eine verbesserte Berücksichtigung der Arbeit der länderspezifischen Konfigurationen der PBC im VN-SR ein und schlug mehrmals vor, die Vorsitzenden dieser länderspezifischen Konfigurationen, wenn sie selbst nicht Mitglieder des VN-SR sind, an den geschlossenen Konsultationen des VN-SR zu den Ländern auf der Tagesordnung der PBC teilnehmen zu lassen. Zwar konnte dies aufgrund des Widerstands der P-5 nicht erreicht werden, die SR-Mitglieder einigten sich jedoch über österreichische Forderung darauf, dass der VN-SR mit länderspezifischen Konfigurationen ,informelle interaktive Dialoge“ führen werde. Ein solcher Dialog fand in Folge bereits mit dem Vorsitzenden der länderspezifischen Konfiguration zu Liberia statt.

Der im September 2010 erschienene Bericht des VN-GS zur Beteiligung und Mitwirkung von Frauen am Peacebuilding (S/2010/466), der einen Aktionsplan für eine verstärkte Partizipation und Berücksichtigung von Frauen im Peacebuilding vorsieht, wurde in der Debatte des VN-SR am 13. Oktober 2010 und der dabei angenommenen Vorsitzerklärung (S/PRST/2010/20) behandelt. In den Verhandlungen dieser Vorsitzerklärung setzte sich Österreich für eine Betonung der Schlüsselrolle von Frauen im Peacebuilding und deren vollständige und gleichberechtigte Teilnahme an Friedensprozessen ein. Ein von Österreich unterstützter Aufruf zur Umsetzung des Berichts des VN-GS und des Aktionsplans konnte zwar aufgrund des Widerstands einiger ständiger SR-Mitglieder nicht durchgesetzt werden, dafür wird der Bericht vom VN-SR wohlwollend zur Kenntnis genommen.

Gemäß ihrer Gründungsresolutionen (SR-Resolution 1945 (2005) und A/RES/60/180) sollte die PBC fünf Jahre nach ihrer Einrichtung einer Überprüfung (sog. „PBC Review“) unterzogen werden. Die Ständigen Vertreter Irlands und Südafrikas (für die VN-GV) und Mexikos (für den VN-SR) waren vom Präsidenten der 64. Generalversammlung als Ko-Fazilitatoren für diesen Prozess ernannt worden. Im Sommer 2010 wurde die Überprüfung abgeschlossen und der Abschlussbericht der drei Ko-Fazilitatoren veröffentlicht. 
In diesem wird u.a. festgehalten, dass Peacekeeping und Peacebuilding keine aufeinanderfolgende, sondern eine gleichzeitige Behandlung erfordern und eine Einbindung der PBC in die Erstellung und Erneuerung von Mandaten von FEO der VN sowie in deren Abzug wünschenswert wäre. Am 29. Oktober 2010 nahmen der VN-SR und die VN-GV gleichlautende Resolutionen (SR-Resolution 1947 (2010), A/RES/65/7) an, in denen der Abschlussbericht über die PBC-Review begrüßt wird, alle relevanten VN-Akteure aufgerufen werden, die Empfehlungen des Abschlussberichts umzusetzen und eine weitere Überprüfung in fünf Jahren festgelegt wird. Im Laufe der Verhandlungen waren v.a. die P-5 bemüht, die Arbeitsbereiche des VN-SR und der PBC klar getrennt zu halten.

\section{Herrschaft des Rechts und Rechtsstaatlichkeit}

Die Stärkung des Völkerrechts und der Rechtsstaatlichkeit („Rule of Law“), mit anderen Worten, das Handeln in den zwischenstaatlichen Beziehungen nach Maßgabe des Völkerrechts als Grundlage für das Funktionieren des bestehenden internationalen Systems, war ein „Leitmotiv“ der österreichischen SR-Mitgliedschaft. Zur Vorbereitung startete Österreich bereits im Jahr 2004 eine Initiative zu Rolle des VN-SR bei der Stärkung der Herrschaft des Rechts und organisierte eine Reihe von Podiumsdiskussionen in New York und einen Retreat in Alpbach im August 2007. Der im April 2008 präsentierte Abschlussbericht „Der VN-Sicherheitsrat und die Herrschaft des Rechts“ enthielt 17 konkrete Empfehlungen, wie der VN-SR in seiner Tätigkeit die Herrschaft des Rechts fördern könnte, um ein auf Regeln beruhendes internationales System zu stärken, deren Umsetzung im Oktober 2010 diskutiert wurde. Österreich arbeitete konsequent daran, die Rechtsstaatlichkeit und das Völkerrecht in der täglichen Arbeit des VN-SR zu stärken. In zahlreichen Resolutionen, Erklärungen und Dokumenten des VN-SR ist die österreichische Handschrift zur Stärkung der Rechtsstaatlichkeit, der Menschenrechte und des humanitären Völkerrechts zu finden. So hat Österreich etwa erstmals erreicht, dass der VN-SR die Konfliktparteien in Gaza und Sri Lanka klar zur Einhaltung des humanitären Völkerrechts aufgefordert hat. Mit den durch die Annahme von SR-Resolution 1904 (2009) erzielten verfahrensrechtlichen Verbesserungen im Rahmen des von Österreich geleiteten 1267-Sanktionenkomitees (Al-Qaida/Taliban) und der Einführung einer unabhängigen Ombudsperson (nähere Ausführungen siehe Kapitel III.9) hat Österreich einen Beitrag zur Stärkung der Rechtsstaatlichkeit innerhalb des VN-SR geleistet. Als weiteres Beispiel dienen die SR-Resolutionen zur Bekämpfung der Piraterie vor der Küste Somalias, in denen sich Österreich für die Entwicklung bzw. Stärkung rechtsstaatlicher Institutionen der Staaten der Region und die Einhaltung des Völkerrechts und der Menschenrechte (und insbesondere des Rechts auf ein faires Verfahren) in der strafrechtlichen Verfolgung Piraterie-Verdächtiger eingesetzt hat. Auch in der unter österrei- 
chischem Vorsitz angenommenen SR-Resolution 1894 (2009) zum Schutz der Zivilbevölkerung in bewaffneten Konflikten wird der Einhaltung des humanitären Völkerrechts, der Menschenrechte sowie des Flüchtlingsrechts durch alle Konfliktparteien sowie den Konsequenzen der Nicht-Einhaltung ein großer Stellenwert eingeräumt (nähere Ausführungen siehe Kapitel III.1; für die österreichischen Bemühungen zur Stärkung der Transparenz der Arbeitsmethoden des VN-SR, siehe Kapitel III.12).

Ein Höhepunkt war die am 29. Juni 2010 von Mexiko in enger Zusammenarbeit mit Österreich (seit vier Jahren erstmals wieder) abgehaltene offene Debatte des VN-SR zur Stärkung der Rechtsstaatlichkeit. Die als Ergebnis der Debatte angenommene Vorsitzerklärung (S/PRST/2010/11) konnte durch österreichische Vorschläge zum Schutz von Zivilisten, Kampf gegen Straflosigkeit und eine Referenz auf die IStGH-Überprüfungskonferenz in Kampala wesentlich gestärkt werden. Der VN-GS wurde aufgefordert, binnen 12 Monaten einen Bericht vorzulegen, in dem die Umsetzung früherer Empfehlungen (vgl. Bericht S/2004/616) und weitere Schritte zur Förderung der Rechtsstaatlichkeit in Konflikt- und Post-Konfliktsituationen untersucht werden sollen. In dieser Debatte betonte Österreich u.a. die Notwendigkeit der Bekämpfung der Straflosigkeit für internationale Verbrechen sowie die Rolle des IStGH, das Erfordernis der Wiedergutmachungen für die Opfer und die Nützlichkeit von Sanktionen, um die Einhaltung des Völkerrechts sicherzustellen.

In der unter dem Vorsitz Frankreichs am 30. Jänner 2009 abgehaltenen geschlossenen SR-Debatte zur Einhaltung des humanitären Völkerrechts forderte Österreich verstärkte Anstrengungen im Kampf gegen die Straflosigkeit und schlug eine Ausweitung des zum Schutz von Kindern in bewaffneten Konflikten bestehenden Mechanismus auf andere Bereiche des humanitären Völkerrechts vor.

\section{Internationale Tribunale und Gerichtshöfe}

Österreich unterstützt traditionell die internationale Strafgerichtsbarkeit und hat sich stets im Rahmen der VN und des IStGH für eine Bekämpfung der Straflosigkeit eingesetzt. Daher war es nur konsequent, dass Österreich den Vorsitz der Informellen Arbeitsgruppe des VN-SR für Internationale Tribunale übernahm. Hauptaufgabe dieser Arbeitsgruppe ist es, die Arbeit des Jugoslawien- und Ruanda-Tribunals (ICTY, ICTR) zu unterstützen und einen Mechanismus vorzubereiten, der die Restfunktionen der Tribunale übernehmen soll. Nach mehr als zweijährigen Verhandlungen konnte schließlich unter österreichischem Vorsitz eine Einigung erzielt werden: Am 22. Dezember 2010 nahm der VN-SR die von Österreich eingebrachte SR-Resolution 1966 (2010) zur Errichtung des Restmechanismus an. Die Annahme der Resolution stellt einen wichtigen Schritt und eine klare Botschaft des VN-SR gegen die Straflosigkeit dar. Durch den Restmechanismus, der einen Standort in Den Haag (Niederlande) und in Arusha (Tansania) haben wird, wird 
sichergestellt, dass gesuchte Kriegsverbrecher wie Ratko Mladic oder Félicien Kabuga auch nach Schließung der Tribunale vor ein internationales Gericht gestellt werden. Neben Gerichtsverfahren wird der Restmechanismus u.a. die Überwachung der Strafvollstreckung, den Zeugen- und Opferschutz, die Überprüfung von Urteilen und die Verwaltung der Archive übernehmen. Bei den rechtlich und politisch komplexen Resolutionsverhandlungen, welche vermutlich zu den längsten der Geschichte des VN-SR zählen, gelang es, in allen umstrittenen Fragen Kompromisslösungen zu finden. Österreich vermittelte als Vorsitz zwischen den fünf ständigen SR-Mitgliedern und war bemüht, insbesondere zwischen den Vereinigten Staaten von Amerika und der Russischen Föderation eine Annäherung der Positionen zu erreichen. Obwohl sich die Russische Föderation letztlich bei der Annahme der Stimme enthielt, begrüßte sie in einer Erklärung ausdrücklich den Beitrag Österreichs, diesen „sehr schwierigen Kompromiss“ zu erzielen. Als Vorsitz der Arbeitsgruppe brachte Österreich während seiner Mitgliedschaft weitere neun SR-Resolutionen zur Unterstützung der Arbeit der VN-Straftribunale ein (vgl. SR-Resolutionen 1877, 1878, 1900 und 1901 (2009) und 1915, 1931, 1932, 1954 und 1955 (2010)).

\section{Wahrung des internationalen Friedens und der Sicherheit, Präventive Diplomatie und Mediation}

Am 21. April 2009 fand eine offene Debatte des VN-SR zu Mediation und Streitbeilegung auf Basis eines entsprechenden Berichts des VN-GS (S/2009/189) statt, die breite Unterstützung für den verstärkten Einsatz von Mediation als Instrument des Krisenmanagements durch die VN bzw. im Zusammenwirken mit oder durch Regionalorganisationen zeigte. Der VN-SR nahm eine Vorsitzerklärung (S/PRST/2009/8) an, die über Betreiben Österreichs den VN-GS auffordert, den VN-SR über weitere Bemühungen zur Förderung der Mediation und der friedlichen Streitbeilegung zu informieren.

Unter nigerianischem Vorsitz widmete sich der VN-SR am 16. Juli 2010 in einer offenen Debatte dem Thema „Optimizing the Use of Preventive Diplomacy Tools: Prospects and Challenges in Africa“. Die SR-Mitglieder betonten die Wichtigkeit eines stärkeren Engagements im Bereich Konfliktprävention und riefen in diesem Zusammenhang auch zur Nutzung der komparativen Vorteile regionaler und subregionaler Organisationen in Afrika auf. Die vom VN-SR angenommene Vorsitzerklärung (S/PRST/2010/14) fordert den VN-GS auf, dem VN-SR binnen zwölf Monaten zu berichten, wie die Bemühungen der VN in diesem Bereich innerhalb der Organisation und in Kooperation mit regionalen und subregionalen Organisationen optimiert werden können. Österreich konnte Verweise auf die Bedeutung des Schutzes der Zivilbevölkerung und der Bekämpfung der Straflosigkeit für die Konfliktprävention sowie auf die Notwendigkeit einer stärkeren Einbindung von Frauen im Text verankern. 
Am 23. September 2010 fand unter dem Vorsitz des türkischen Präsidenten Abdullah Gül ein SR-Gipfel zum Thema „Sicherstellung einer effektiven Rolle des VN-SR in der Wahrung des internationalen Friedens und der Sicherheit" statt. Im Zentrum der Diskussion standen eine effizientere Nutzung der bestehenden VN-Instrumente zur Schaffung und Erhaltung von Frieden, eine bessere Kohärenz der vielen Akteure auf regionaler und VNEbene sowie ein stärkeres Augenmerk auf Prävention und Frühwarnmechanismen. Für Österreich nahmen Bundespräsident Heinz Fischer und Bundesminister Michael Spindelegger am Gipfel teil. Als Ergebnis nahm der VN-SR eine Vorsitzerklärung (S/PRST/2010/18) an, in der er sein Bekenntnis zu einer effizienten Erfüllung seiner Aufgaben erneuerte und neue Herausforderungen wie etwa Terrorismus, Proliferation von KLW, organisiertes Verbrechen, Piraterie sowie Drogen- und Menschenhandel anerkennt. Basierend auf einem österreichischen Vorschlag bekräftigte der VN-SR außerdem die wichtige Rolle der Frauen in allen Bereichen der Friedenskonsolidierung.

\section{Verbrechens- und Terrorismusbekämpfung}

Während der österreichischen SR-Mitgliedschaft führte Botschafter Thomas Mayr-Harting den Vorsitz im 1267-Sanktionenkomitee (Al-Qaida/Taliban). Das 1267-Komitee ist für die Umsetzung der Sanktionen gegen Personen und Entitäten, die mit Al-Qaida bzw. den Taliban in Verbindung stehen, zuständig. Auf einer sogenannten „Konsolidierten Liste“ werden rund fünfhundert Personen und Entitäten in Verbindung mit beiden Gruppierungen geführt, die durch Vermögenseinfrierung, Reiseverbote und Waffenembargos sanktioniert werden. Diese Konsolidierte Liste ist ein wichtiges Instrument, um gezielt Sanktionen gegen Terroristen durchzusetzen und somit deren Aktionsradius einzuschränken. Gleichzeitig musste aus Sicht des österreichischen Vorsitzes sichergestellt sein, dass das Komitee-interne Verfahren und die Umsetzung dieser Maßnahmen den internationalen und europarechtlichen Maßstäben an Rechtsstaatlichkeit, Transparenz und Fairness entsprechen. Wichtiger Schritt in diese Richtung war die gemäß SR-Resolution 1822 (2008) bis Mitte 2010 durchgeführte umfassende Überprüfung aller Listeneinträge („Review“). Damit sollte eine Aktualisierung und Dynamisierung der Liste einhergehen; die ordnungsgemäße Durchführung des entsprechenden Verfahrens zur genauen Prüfung jedes einzelnen Falles wurde durch den österreichischen Vorsitz sichergestellt.

Im Zuge des eineinhalb Jahre dauernden Review, der in Kooperation mit allen betroffenen Staaten durchgeführt wurde, wurden 488 Einträge überprüft, 46 Namen von der Liste gestrichen (Streichungen von der Liste setzen die Zustimmung aller Komiteemitglieder voraus), rund 50 weitere Delistinganträge sind noch in Bearbeitung. Angesichts der laufenden Debatte zur Versöhnung mit moderaten Taliban zur Stabilisierung Afghanistans hat dieser an sich technische Überprüfungsprozess an politischer Dynamik gewonnen 
und Österreich auch als Vorsitz politisch gefordert. Bei der Beurteilung der entsprechenden Anträge berücksichtigte das Komitee insbesondere, ob die $\mathrm{zu}$ streichenden Personen der Gewalt entsagten, die Verbindungen zu AlQaida abbrachen und die Afghanische Verfassung respektierten. Letztlich konnten elf „ausgesöhnte“ Taliban von der Liste gestrichen werden.

Mit der Annahme von SR-Resolution 1904 (2009) konnte Österreich in enger Zusammenarbeit mit den Vereinigten Staaten von Amerika und den anderen SR-Mitgliedern zahlreiche weitere verfahrensrechtliche Verbesserungen im 1267-Komitee verankern. Eine wesentliche Neuerung, die dem österreichischen Engagement zu verdanken war, war die Einrichtung einer Ombudsperson zur unabhängigen Überprüfung von Anträgen auf Streichung von der Konsolidierten Liste. Während Personen, die sich zu Unrecht auf der Liste geführt sahen, bislang keine Möglichkeit hatten, eine unabhängige Überprüfung ihres Falles herbeizuführen, wird durch die Einführung der Ombudsperson nun ein klares Verfahren in einem vorgezeichneten Zeitrahmen festgelegt. Die durch den VN-GS mit diesem Posten beauftragte kanadische Richterin Kimberly Prost hat Anfang Juli 2010 ihre Arbeit aufgenommen und bis Jahresende 2010 mit der Bearbeitung der ersten fünf Fälle begonnen. Weitere Bestimmungen in SR-Resolution 1904 (2009) sehen vor, dass Staaten, die einen neuen Eintrag für die Konsolidierte Liste vorschlagen, dazu umfassendere und exaktere Informationen als bislang liefern sollen. Solche Einträge sollen nunmehr eine klare Identifikation der betroffenen Person bzw. Entität beinhalten, um die Umsetzung der Sanktionsmaßnahmen durch innerstaatliche Behörden zu erleichtern. Zusätzlich erhalten die KomiteeMitglieder mehr Zeit als bisher, um einen Neueintrag zu prüfen und gegebenenfalls ergänzende Informationen bereit zu stellen. Weiters legt SR-Resolution 1904 (2009) fest, dass auch nach der generellen Überprüfung der Konsolidierten Liste gemäß SR-Resolution 1822 (2008) eine kontinuierliche Aktualisierung der Liste erfolgen soll, um zu vermeiden, dass Einträge mit unzureichenden Identifikatoren oder von verstorbenen Personen bzw. aufgelösten Entitäten auf der Liste aufscheinen. Darüber hinaus ist vorgesehen, zukünftig jeden Eintrag alle drei Jahre einer generellen Überprüfung zu unterziehen. Auch die Straffung der Fristen im Rahmen der Komitee-Arbeit soll zu einem verbesserten Verfahren und zur Aktualität der Liste beitragen. Ebenso sollen begründungslose Ablehnungen von Entscheidungen, insbesondere von Delistinganträgen, hintan gehalten werden.

Im Sinne der österreichischen Bestrebungen zur Stärkung der Herrschaft des Rechts in den internationalen Beziehungen wurde durch diese Verfahrensverbesserungen versucht, international üblichen Standards der Rechtsstaatlichkeit besser Rechnung zu tragen. In den vergangenen Jahren haben Gerichte in diversen Staaten und insbesondere die europäischen Gerichte Kritik an der Umsetzung der VN-Sanktionen geübt. So forderten die EUGerichte von der Europäischen Kommission, die Sanktionsbeschlüsse des VN-SR im Rahmen der EU mit Wirksamkeit für alle EU-Mitgliedstaaten 
umsetzt, immer deutlicher die Beachtung grundlegender rechtsstaatlicher Verfahrensregeln. In diesem Zusammenhang beschäftigten sie sich auch mit deren Berücksichtigung durch den VN-SR. Vor diesem Hintergrund kam es im Oktober 2009 auf österreichische Initiative auch zu einem Besuch einer Delegation des 1267-Komitees bei den EU-Institutionen in Brüssel. Mit SRResolution 1904 (2009), die von Österreich wesentlich mitgestaltet wurde, hat der VN-SR die rechtsstaatlichen Verfahrensstandards im 1267-Komitee maßgeblich verbessert und damit die Wirksamkeit und die Legitimität im Kampf gegen die terroristische Bedrohung durch Al-Qaida und die Taliban erhöht.

\subsection{Antiterrorismuskomitee}

Ein weiteres Unterorgan des VN-SR, welches sich mit Terrorismusbekämpfung beschäftigt, ist das durch SR-Resolution 1373 (2001) eingerichtete Antiterrorismuskomitee (CTC). SR-Resolution 1373 (2001) enthält weitreichende Verpflichtungen zur Kriminalisierung jeglicher Art von terroristischen Aktivitäten und der Unterbindung der Finanzierung von Terrorismus. Das CTC soll auf Ebene der VN-Mitgliedstaaten die Umsetzung dieser Maßnahmen überwachen bzw. hierbei assistieren. Zur Unterstützung ist dem CTC ein Exekutivdirektorium (CTED) beigefügt, welches aus rund 30 Experten besteht, die u.a. Staatenbesuche und -evaluierungen vornehmen und technische Unterstützung auf bilateraler oder multilateraler Basis vermitteln. $\mathrm{Zu}$ betonen ist in diesem Zusammenhang die bewährte Arbeitsteilung zwischen der Vermittlung technischer Unterstützung durch das CTED und der tatsächlichen Leistung durch die Unterabteilung für Terrorismusbekämpfung von UNODC und anderer Anbieter technischer Unterstützung.

Österreich misst einer effektiven Terrorismusbekämpfung auf rechtsstaatlicher Grundlage hohe Bedeutung bei. Dem Schutz der Menschenrechte kommt dabei eine zentrale Rolle zu. Österreich plädierte in diesem Sinne für ein vermehrtes Engagement des CTC und CTED im Bereich der Wahrung von Menschenrechten bei der Terrorismusbekämpfung. So konnte sich Österreich auch bei einem Briefing der VN-Hochkommissarin für Menschenrechte Navanethem Pillay, zu den menschenrechtlichen Aspekten der Terrorismusbekämpfung im CTC im Oktober 2009 konstruktiv einbringen. Auch im Rahmen der Mandatserweiterung des CTED durch SR-Resolution 1963 (2010) setzte sich Österreich für eine Stärkung des menschenrechtlichen Aspekts in der Arbeit des CTC und des CTED ein. Weiterer Schwerpunkt Österreichs war die Förderung einer transparenteren Arbeitsweise der Antiterrorismuskomitees, wie etwa durch die regelmäßige Abhaltung von offenen Briefings für alle VN-Mitgliedstaaten.

Insgesamt betonte Österreich während seiner SR-Mitgliedschaft die Wahrung der Kohärenz und Synergie zwischen den VN-Terrorismusbekämpfungsstrukturen, nämlich dem 1267-Sanktionenkomitee (Al-Qaida/Taliban), 
dem CTC, dem 1540-Komitee (siehe dazu Kapitel III.10), sowie mit der Unterabteilung von UNODC und im Rahmen der VN-Anti-Terrorismus-TaskForce (CTITF). Österreich beteiligte sich aktiv an der Erarbeitung von Empfehlungen für eine verstärkte Zusammenarbeit der drei AntiterrorismusKomitees, die u.a. anlässlich der gemeinsamen halbjährlichen Briefings der Vorsitzenden des 1267-, CTC und des 1540-Komitees im VN-SR erarbeitet wurden. Die Expertengruppen der drei Komitees sollen dementsprechend nach Möglichkeit zunehmend ihre Länderbesuche und Teilnahmen an Workshops koordinieren und auch gemeinsam abhalten und gegenseitig Informationen austauschen. Österreich hob außerdem das Thema der Ko-Lokation der drei Expertengruppen hervor und erinnerte an den in SR-Resolution 1904 (2009) enthaltenen Aufruf an den VN-GS, die drei Gruppen möglichst rasch gemeinsam anzusiedeln.

\subsection{Transversale Bedrohungen}

Für Österreich war neben der Arbeit in den Antiterrorismuskomitees auch eine gebührende Berücksichtigung der sog. „,transversalen Bedrohungen“ im VN-SR von großer Bedeutung. Grenzüberschreitende Sicherheitsbedrohungen wie organisierte Kriminalität, Korruption oder Drogenhandel haben negative Auswirkungen auf nachhaltige Friedens- und Stabilisierungsbemühungen und werden vom VN-SR daher mit zunehmender Sorge beobachtet. Im Lichte der Globalisierung der verschiedenen Formen des organisierten Verbrechens gewinnt das in Wien ansässige UNODC, welches einen integrierten Ansatz bei der Bekämpfung von grenzüberschreitender organisierter Kriminalität verfolgt und eine entscheidende Rolle bei der Umsetzung der VN-Konvention gegen die grenzüberschreitende organisierte Kriminalität (UNTOC) spielt, zusätzlich an Bedeutung. UNODC hat seine Bemühungen intensiviert, den VN-Mitgliedstaaten verstärkt bedarfsorientierte Unterstützung im Hinblick auf die Bewältigung der neuen Herausforderungen im Sicherheitsbereich - organisierte Kriminalität, Drogenhandel, Terrorismus, Menschenhandel - anzubieten. In all diesen Bereichen verfügt UNODC über Erfahrungen und Expertise.

Die Tatsache, dass grenzüberschreitende Kriminalität und insbesondere der Drogenhandel den internationalen Frieden und die Sicherheit gefährden können, veranlasste den VN-SR auch dazu, sich mit diesen Fragen in thematischen Debatten zu befassen. Am 8. Dezember 2009 widmete sich der VN-SR unter dem Vorsitz von Burkina Faso erstmals in einer offenen Debatte, an der auch UNODC-Exekutivdirektor Costa teilnahm, dem Thema „Drogenhandel als Bedrohung der internationalen Sicherheit" mit besonderem Fokus auf Westafrika. Eine in der Debatte angenommene Vorsitzerklärung (S/PRST/ 2009/32) qualifiziert Drogenhandel in einigen Situationen als Bedrohung der internationalen Sicherheit, anerkennt die wichtige Rolle der verschiedenen VN-Gremien (darunter auch UNODC) im Kampf gegen den Drogenhandel 
und ruft den VN-GS auf, den Kampf gegen den Drogenhandel zum integralen Bestandteil von Konfliktpräventionsstrategien und Konfliktanalysen sowie in der Überprüfung von VN-Missionen und im Peacebuilding zu machen.

Auf dieser Debatte aufbauend, erhielt der VN-SR am 24. Februar 2010 auf Einladung des französischen Vorsitzes ein Briefing von UNODC-Exekutivdirektor Costa zu transnationalen Bedrohungen des Weltfriedens wie organisierter Kriminalität und Drogenhandel. Der VN-SR nahm eine Vorsitzerklärung (S/PRST/2010/4) an, die u.a. die Möglichkeit regelmäßiger Briefings von UNODC im VN-SR vorsieht. Der VN-GS wurde zudem aufgerufen, Drogenhandel und grenzüberschreitende Kriminalität als Faktoren in Konfliktpräventionsstrategien, Konfliktanalysen sowie in der Planung und Bewertung von integrierten VN-Missionen zu berücksichtigen und dem VN-SR entsprechende Analysen zu unterbreiten. Auf österreichischen Vorschlag konnte erreicht werden, dass neben dem Drogenhandel auch die grenzüberschreitende organisierte Kriminalität generell als integraler Bestandteil von Konfliktanalysen und der Überprüfung von VN-Missionen erwähnt wird. In seiner Wortmeldung betonte Österreich auch die Dringlichkeit der Korruptionsbekämpfung und verwies auf die neu eingerichtete IACA in Laxenburg. Insgesamt trugen die Debatten zu transversalen Bedrohungen zu einer erhöhten Visibilität der Arbeit des in Wien ansässigen UNODC innerhalb des VNSystems bei. Dies kann auch als Erfolg der österreichischen Bemühungen zur Stärkung des Amtssitzes Wien gewertet werden.

\section{Nukleare Abrüstung und Non-Proliferation}

Das hochrangigste Treffen des VN-SR in der Zeit der österreichischen SRMitgliedschaft war der auf Ebene der Staats- und Regierungschefs abgehaltene SR-Gipfel vom 24. September 2009 unter dem Vorsitz von US-Präsident Obama zu Fragen der nuklearen Abrüstung und nuklearen Non-Proliferation. Mit der an diesem Tag einstimmig angenommenen SR-Resolution 1887 (2009) bekannte sich der VN-SR erstmals zum Ziel einer Welt ohne Nuklearwaffen. Für Österreich nahmen Bundespräsident Heinz Fischer und Bundesminister Michael Spindelegger teil. Bundespräsident Heinz Fischer trat als einziger Redner für eine Nuklearwaffenverbotskonvention ein und betonte u.a., dass Nuklearwaffenstaaten ihre Nuklearwaffenarsenale weiter reduzieren sollten, ein Prozess zum Inkrafttreten des CTBT entworfen werden müsse und die Multilateralisierung des nuklearen Brennstoffzyklus unter IAEOKontrolle eine wertvolle vertrauensbildende Maßnahme darstellen würde. In SR-Resolution 1887 (2009) ruft der VN-SR u.a. die verbleibenden Nichtvertragsparteien des Vertrags über die Nichtverbreitung von Kernwaffen (NPT) auf, letzterem als Nicht-Nuklearwaffenstaat beizutreten und fordert alle Staaten auf, Nukleartestexplosionen zu unterlassen und den CTBT zu unterzeichnen bzw. zu ratifizieren. Weiters ruft er die Abrüstungskonferenz auf, einen Vertrag zum Verbot der Produktion spaltbaren Materials (FMCT) zu 
verhandeln, zeigt sich besorgt über bestehende Herausforderungen des NonProliferationsregimes (in Anspielung auf, jedoch ohne explizite Nennung des Iran und der Demokratischen Volksrepublik Korea) und fordert die Einhaltung seiner bestehenden Sanktionenresolutionen. Der VN-SR ruft alle Staaten auf, strengere Exportbestimmungen für proliferationskritische Teile des nuklearen Brennstoffzyklus anzunehmen. Der IAEO-Gouverneursrat solle ehest möglich Maßnahmen zur Multilateralisierung des nuklearen Brennstoffzyklus erlassen.

Österreich brachte sich intensiv in die Resolutionsverhandlungen ein und konnte neben der Verankerung bedeutender Abrüstungs- und Non-Proliferationsmaßnahmen außerdem gegen einige Widerstände sicherstellen, dass sich die Aussagen des VN-SR zur Förderung der friedlichen Nutzung der Nuklearenergie explizit nur auf jene Staaten beziehen, die sich für diese Energieform entschieden haben, wobei überdies auf die Notwendigkeit hingewiesen wird, höchste internationale Sicherheitsstandards zu beachten.

\subsection{0-Komitee}

Während seiner SR-Mitgliedschaft hatte sich Österreich im 1540-Komitee betreffend die Nichtweiterverbreitung von Massenvernichtungswaffen an nichtstaatliche Akteure (Terroristen) konsequent für Verbesserungen im Bereich der Non-Proliferation eingesetzt. So konnte sich Österreich bei der gemäß SR-Resolution 1810 (2008) vorgesehenen umfassenden Überprüfung des Umsetzungsstands der SR-Resolution 1540 (2004), die am 29. Jänner 2010 abgeschlossen werden konnte, aktiv durch zahlreiche konkrete Vorschläge und Empfehlungen einbringen. Zentraler Bestandteil der Überprüfung waren erstmalig durchgeführte offene Komiteesitzungen, die auf eine österreichische Initiative zurückgingen und zu denen VertreterInnen aller VN-Mitgliedstaaten und ausgewählter internationaler und regionaler Organisationen und Entitäten eingeladen waren. Weiters gelang es wesentliche österreichische Interessen und Themenschwerpunkte, wie die Notwendigkeit einer strengen Einhaltung der Menschenrechte, Verfahrensrechte und rechtsstaatlichen Garantien sowie, erstmals im Kontext der SR-Resolution 1540 (2004), die Problematik des Individualrechtsschutzes, in einem VNDokument zu verankern.

Im Rahmen der Tätigkeit Österreichs als Koordinator der 1540-Arbeitsgruppe zur Kooperation mit Internationalen Organisationen organisierte Österreich gemeinsam mit dem VN-Abrüstungsbüro (UNODA) am 15. und 16. Dezember 2010 in der Wiener Hofburg eine Expertenkonferenz zur Kooperation mit internationalen, regionalen und subregionalen Organisationen (IRO) bei der Umsetzung von SR-Resolution 1540 (2004). Mit dieser im 1540-Kontext erstmaligen Konferenz, an der insgesamt 25 IROs, der 1540-Komiteevorsitzende, mehrere Komiteevertreter und 1540-Experten sowie Vertreter von UNODA teilnahmen, leistete Österreich einen konkreten Beitrag zur Umsetzung der 
SR-Resolution 1810 (2008), in der das 1540-Komitee zur verstärkten Zusammenarbeit mit IROs aufgerufen wird. Vorrangiger Zweck der Konferenz war die Verbesserung der Kooperation zwischen den IROs selbst und dem 1540-Komitee, sowie die Nutzung von Synergien der komplementären Mandate zwischen den IROs bei der Umsetzung der SR-Resolution 1540 (2004). Die Konferenz stellte ein weiteres Beispiel für das kontinuierliche Engagement Österreichs im Bereich der Nichtverbreitung von Massenvernichtungswaffen dar. Österreich kündigte im Sinne der Nachhaltigkeit dieser österreichischen Initiative seine Bereitschaft für die Abhaltung ähnlicher Treffen an.

Die Entscheidung vom Herbst 2010 zur Ansiedlung von zwei neuen abrüstungsrelevanten Institutionen in Wien (Verbindungsbüro für UNODA, Wiener Zentrum für nukleare Abrüstung und nukleare Non-Proliferation) stellte eine zusätzliche wichtige Erweiterung der in Wien bereits bestehenden Kompetenzen (vgl. IAEO, CTBT-Vorbereitungskommission) dar und ist ein sichtbarer Erfolg der österreichischen Arbeit in diesem Bereich. Damit konnte während der österreichischen SR-Mitgliedschaft eine schon länger bestehende Idee umgesetzt werden.

\section{Arbeitsmethoden des Sicherheitsrats}

Österreich hat sich im VN-SR sowie im Rahmen der Informellen Arbeitsgruppe des VN-SR zu Dokumentation und anderen Verfahrensfragen für eine Stärkung der Transparenz in der Arbeit des VN-SR eingesetzt. In der am 22. April 2010 abgehaltenen offenen Debatte zu den Arbeitsmethoden des VN-SR begrüßte Österreich die Abhaltung sog. ,informeller interaktiver Dialoge“ (etwa zu Sri Lanka), sprach sich für eine verstärkte Interaktion des VN-SR mit den VN-Mitgliedstaaten sowie Regionalorganisationen aus und forderte zum wiederholten Mal eine verbesserte Zusammenarbeit des VN-SR mit der PBC (alle Vorsitzenden der länderspezifischen Konfigurationen der PBC sollten an den Konsultationen des VN-SR zum jeweiligen Land auf der Tagesordnung der PBC teilnehmen können).

Mit diesen Fragen hat sich auch die Informelle Arbeitsgruppe des VN-SR zu Dokumentation und anderen Verfahrensfragen unter dem Vorsitz Japans befasst, wobei das wichtigste Ergebnis dieser Arbeitsgruppe die Überarbeitung der Note des SR-Präsidenten (S/2006/507) über die Arbeitsmethoden des VN-SR war (die überarbeitete Note S/2010/507 wurde vom VN-SR am 27. Juli 2010 angenommen); dies entsprach dem österreichischen Anliegen nach mehr Transparenz gegenüber den Nicht-Mitgliedern des VN-SR. Neue Elemente dieser Note sind u.a. die Bestimmungen zu Missionen des VN-SR, zu einem regelmäßigen Dialog des VN-SR mit der PBC einschließlich der Möglichkeit, einen informellen Dialog mit den länderspezifischen Konfigurationen zu führen, sowie die Referenz zu den informellen Dialogen des VN-SR. 


\section{Zusammenarbeit der VN mit Regionalorganisationen}

Zum Thema der Zusammenarbeit der VN mit Regionalorganisationen fanden mehrere Sitzungen des VN-SR statt. Die am 13. Jänner 2010 vom chinesischen Vorsitz abgehaltene Debatte zur Kooperation der VN mit regionalen und subregionalen Organisationen in der Erhaltung des internationalen Friedens und der Sicherheit unter Beteiligung von elf Regionalorganisationen stellte das bisher größte Zusammentreffen des VN-SR mit Regionalorganisationen dar. In der als Sitzungsergebnis angenommenen Vorsitzerklärung (S/PRST/2010/1) drückt der VN-SR seine Absicht aus, informelle interaktive Dialoge mit regionalen und subregionalen Organisationen abzuhalten. Auf Anregung Österreichs unterstreicht die Vorsitzerklärung die Rolle der Regionalorganisationen in der Umsetzung von VN-SR Resolutionen.

In einer von Österreich initiierten öffentlichen Sitzung informierte EU-HV Ashton den VN-SR am 4. Mai 2010 über die Zusammenarbeit zwischen den VN und der EU auf dem Gebiet der Erhaltung des Weltfriedens. EU-HV Ashton verwies darauf, dass EU und VN gleiche Ziele verfolgen, die EU der kollektiv größte Beitragszahler der VN sei und sich die Partnerschaft zwischen der EU und den VN in den letzten Jahren bedeutend verstärkt habe. Österreich war durch Bundesminister Michael Spindelegger vertreten, der die Bedeutung der Kooperation zwischen VN und EU hervorhob und unterstrich, dass der Vertrag von Lissabon die internationale Vertretung der EU erleichtert habe. Weiters verwies Bundesminister Michael Spindelegger auf die wichtige Rolle der EU bei der Umsetzung von SR-Resolutionen zum Schutz der Zivilbevölkerung sowie zur Rolle von Frauen in der Stärkung von Frieden und Sicherheit.

Im Zeitraum 2009/10 widmete sich der VN-SR viermal der Zusammenarbeit zwischen den VN und der AU. Im Rahmen der offenen Debatte am 18. März 2009 präsentierte der ehemalige italienische Premierminister Romano Prodi dem VN-SR den unter seinem Vorsitz erstellten Expertenbericht über Modalitäten der Unterstützung von FEO der AU mit VN-Mandat (sog. „ProdiBericht“). Der Bericht betont die Notwendigkeit einer Stärkung der strategischen Partnerschaft zwischen den VN und der AU und der Schaffung entsprechender Koordinierungsmechanismen. Ein zweiter Schwerpunkt betrifft den Kapazitätenaufbau der AU in den Bereichen Friedenssicherung, Frühwarnung und Konfliktprävention. Drittens enthält der Bericht Vorschläge für die Finanzierung der von der AU durchgeführten FEO mit VN-Mandat. Unter den SR-Mitgliedern bestand v.a. Divergenz hinsichtlich der Finanzierung von FEO der AU aus VN-Pflichtbeiträgen. Der VN-SR nahm eine Vorsitzerklärung (S/PRST/2009/3) an, in welcher der VN-GS aufgefordert wird, die verschiedenen Modalitäten zur Unterstützung der AU einschließlich der Empfehlungen des Prodi-Berichts näher zu prüfen und dem VN-SR darüber zu berichten. Österreich setzte sich in dieser sowie in den weiteren Debatten zum Thema für eine offene Diskussion sämtlicher Vorschläge für eine verbesserte Zusammenarbeit zwischen VN und AU ein. 
Am 26. Oktober 2009 fand auf der Grundlage des Folgeberichts des VN-GS (S/2009/470) zum Bericht des Prodi-Panels eine weitere offene Debatte über die Unterstützung von AU-geführten FEO mit VN-Mandat statt. Der Bericht des VN-GS geht auf die Möglichkeiten einer verbesserten Zusammenarbeit zwischen dem VN-SR und dem Friedens- und Sicherheitsrat der AU (AUPSC) ein und betont die Notwendigkeit von kohärenten Entscheidungen beider Gremien sowie deren verstärkter Zusammenarbeit. Weiters unterstreicht der Bericht die Wichtigkeit einer engeren Kooperation zwischen VN-Sekretariat und AU-Kommission und plädiert für nachhaltigen Kapazitätenaufbau der AU. Hinsichtlich der Frage der Finanzierung AU-geführter und VNmandatierter FEO werden verschiedene Optionen analysiert: Pflichtbeiträge der AU-Mitgliedstaaten, Beiträge von AU-Truppenstellern, freiwillige Beiträge wie etwa der EU im Wege der Afrikanischen Friedensfazilität (APF), sowie VN-Pflichtbeiträge oder eine Kombination dieser Finanzierungsmechanismen. Gegen die von der AU geforderte Finanzierung von AU-Operationen aus VN-Pflichtbeiträgen bestand in der Debatte unter den SR-Mitgliedern weiterhin Widerstand. Österreich betonte die Bedeutung der trilateralen Partnerschaft zwischen AU, VN und EU in der Beilegung von Konflikten sowie im Hinblick auf Menschenrechte und den Schutz der Zivilbevölkerung. In einer weiteren Vorsitzerklärung (S/PRST/2009/26) beschloss der VN-SR, sich weiterhin mit der Thematik zu befassen.

Die für die logistische Unterstützung von FEO zuständige UGS Susana Malcorra berichtete dem VN-SR am 12. April 2010 in geschlossenen Konsultationen über Fortschritte in der Zusammenarbeit zwischen VN und AU und erwähnte insbesondere die Zusammenfassung aller in Addis Abeba angesiedelten VN-Präsenzen zu einem integrierten Büro als wichtigen Schritt zur Stärkung der Effizienz der VN-Repräsentation. Österreich erneuerte sein Angebot, die AU bei der Einrichtung eines Büros am VN-Amtssitz Wien zu unterstützen. Im Rahmen der offenen SR-Debatte am 22. Oktober 2010 stellte der VN-GS einen Bericht über Möglichkeiten zur Stärkung der strategischen Partnerschaft zwischen den VN und der AU in Aussicht. AU-Kommissar für Frieden und Sicherheit Ramtane Lamamra forderte eine dauerhafte Lösung der Finanzierungsfrage von AU-geführten FEO. Im Anschluss an die Debatte nahm der VN-SR eine Vorsitzerklärung (S/PRST/2010/21) an, in der die Notwendigkeit einer nachhaltigen, vorhersehbaren und flexiblen Finanzierung von FEO der AU unterstrichen wird.

Zusätzlich fanden jährliche Briefings des jeweiligen OSZE-Vorsitzes über die Prioritäten des Vorsitzlandes statt. 


\section{Der österreichische Vorsitz im Sicherheitsrat im November 2009}

Im November 2009 übernahm Österreich den Vorsitz im VN-SR. Die SR-Mitglieder nehmen diese Aufgabe im monatlich wechselnden Turnus wahr, wobei die Mitgliedstaaten in der Reihenfolge des englischen Alphabets zum Zuge kommen. Die Liste wird über die Jahre hinweg fortlaufend geführt; hinzukommende nicht-ständige Mitglieder werden jeweils eingefügt, ausscheidende gestrichen. Ob ein gewähltes Mitglied den Vorsitz während der 24 Monate seiner SR-Mitgliedschaft ein- oder zweimal ausübt, hängt davon ab, an welchem Punkt die alphabetische Liste zum Zeitpunkt seines Eintritts in den VN-SR gerade angekommen war. Österreich hatte den SR-Vorsitz diesmal - anders als 1991/92 - nur einmal inne, konnte sich auf diesen aber dafür umso gründlicher vorbereiten.

Die Aufgaben des Vorsitzes umfassen die Vorbereitung des Arbeitsprogramms, die Leitung der öffentlichen sowie geschlossenen Sitzungen des VN-SR sowie die Wahrnehmung der mit der Funktion des Präsidenten verbundenen Verpflichtungen gegenüber dem VN-Sekretariat, der VN-Mitgliedschaft sowie den Medien und der Zivilgesellschaft.

Während des österreichischen SR-Vorsitzes standen Debatten zum Irak, zu Bosnien und Herzegowina, zu Somalia, zur Demokratischen Republik Kongo, zum Sudan, zu Nepal, zu Guinea-Bissau und zum Nahen Osten auf der Tagesordnung des VN-SR. Mit SR-Resolution 1895 (2009) wurde die Autorisierung der Operation der EU in Bosnien und Herzegowina (EUFOR Althea) um ein weiteres Jahr verlängert; SR-Resolution 1896 (2009) verlängerte die Sanktionen betreffend die Demokratische Republik Kongo um ein Jahr; und SR-Resolution 1897 (2009) verlängerte die Autorisierung der Maßnahmen im Kampf gegen die Piraterie vor der Küste von Somalia um zwölf Monate.

Unter Vorsitzführung von Bundesminister Michael Spindelegger verabschiedete der VN-SR am 11. November 2009 einstimmig SR-Resolution 1894 (2009) über den Schutz der Zivilbevölkerung in bewaffneten Konflikten.

Während des österreichischen Vorsitzes kam es - nach der Unterzeichnung eines Militärabkommens zwischen den Vereinigten Staaten von Amerika und Kolumbien - zu starken Spannungen zwischen Venezuela und Kolumbien. Beide Staaten befassten in dieser Situation den österreichischen SRVorsitz, der in informellen Gesprächen zur Zufriedenheit beider Seiten zur Deeskalation beitragen konnte.

In seiner Eigenschaft als SR-Präsident stellte Botschafter Thomas Mayr-Harting am 12. November 2009 dem Plenum der VN-GV den Jahresbericht über die Tätigkeit des VN-SR vor und gab am 30. November 2009 eine Erklärung bei der Sonderveranstaltung aus Anlass des internationalen Tages der Solidarität mit dem palästinensischen Volk ab. 


\section{Vorbereitungen}

In enger Abstimmung zwischen der Ständigen Vertretung Österreichs bei den VN in New York (ÖV New York) und der Zentrale des BMeiA in Wien wurde schon zu Beginn des Jahres 2009 entschieden, den österreichischen Vorsitz dem Schutz der Zivilbevölkerung in bewaffneten Konflikten zu widmen. Österreich setzte sich das Ziel, dieses Thema, das ohnehin auf der Tagesordnung des VN-SR im November stand, nicht nur in einer thematischen Debatte zu behandeln, sondern darüber hinaus ein konkretes Ergebnis in Form einer Resolution anzustreben, das einen Fortschritt für die Arbeit der VN zum Schutz von Zivilisten in bewaffneten Konflikten erbringen sollte. Da es zu diesem sensiblen Thema unter einigen SR-Mitgliedern erhebliche Vorbehalte gab, stellte diese Zielsetzung eine große Herausforderung dar. Die letzte SR-Resolution zum Schutz der Zivilbevölkerung in bewaffneten Konflikten (SR-Resolution 1674 (2006)) stammte aus dem April 2006 und wurde von Großbritannien, das im VN-SR als „lead country“ zu dieser Thematik fungiert, verhandelt und eingebracht.

Neben den laufenden Sondierungsgesprächen der ÖV New York wurden zur Vorbereitung zwei Veranstaltungen organisiert. Ein vom BMeiA und BMLVS in Kooperation mit dem IPI im Juni 2009 in Wien durchgeführtes Seminar war dem Thema „The Security Council and the Responsibility to Protect“" gewidmet. Hochrangige VertreterInnen des VN-SR, einschließlich US-Botschafterin Susan Rice, leitende MitarbeiterInnen des VN-Sekretariats, sowie führende Kräfte des Militärs, der Zivilgesellschaft und der Wissenschaft diskutierten in umfassender Weise, welche Rolle dem VN-SR beim Schutz der Zivilbevölkerung zukommt und unter welchen Voraussetzungen ein Einschreiten des VN-SR möglich bzw. notwendig ist. Der österreichische VN Retreat Ende August 2009 in Alpbach setzte sich mit den aktuellen Herausforderungen des VN-SR zum Schutz der Zivilbevölkerung in bewaffneten Konflikten auseinander und bot eine Diskussionsplattform für SR-Mitglieder, Ständige VertreterInnen großer Truppensteller sowie Spitzen des VN-Sekretariats, angeführt von VN-GS Ban Ki-moon, UGS Holmes und UGS Le Roy. Sowohl das IPI-Seminar als auch der AlpbachRetreat lieferten wichtige Erkenntnisse über die Herausforderungen beim Schutz der Zivilbevölkerung und den notwendigen Handlungsbedarf, die in die Erarbeitung eines Resolutionsentwurfs einfließen konnten. Die Arbeiten eines zehnköpfigen Teams von MitarbeiterInnen der ÖV New York sowie der Zentrale zur Vorbereitung von Elementen für einen Resolutionsentwurf begannen bereits unmittelbar nach dem IPI-Seminar. Diese Elemente konnten im Rahmen des Alpbach-Retreats, insbesondere auch mit den Ständigen VertreterInnen der SR-Mitglieder sowie in weiterer Folge mit dem VN-Sekretariat ausgelotet werden. Ein erster konsolidierter Resolutionsentwurf wurde ab September in informellen bilateralen Kontakten der ÖV New York mit SR-Mitgliedern, VertreterInnen des VN-Sekretariats und der Zivilgesellschaft konsultiert. 
Bundesminister Michael Spindelegger brachte sich in den Vorbereitungsprozess von Anfang an ein, indem er Reisen in Hauptstädte der P-5 unternahm und seine Amtskollegen sowie in Wien akkreditierte Botschafter über das österreichische Vorhaben informierte und um Unterstützung ersuchte.

\section{Verhandlung und Annahme der SR-Resolution 1894 (2009)}

Die eigentlichen Verhandlungen des Resolutionsentwurfs im VN-SR in New York begannen am 15. Oktober 2009 und konnten am 9. November $2009 \mathrm{zu}$ einem erfolgreichen Abschluss gebracht werden. In diesem Zeitraum fanden insgesamt zwölf halbtägige Verhandlungsrunden unter österreichischem Vorsitz statt. Während des Verhandlungsprozesses stand der österreichische Vorsitz auch in laufendem Kontakt mit dem VN-Sekretariat und der Zivilgesellschaft. Die Verhandlungen verliefen trotz ihrer Intensität und der sensiblen Materie in konstruktiver Atmosphäre. Letztlich konnte zu beinahe allen wesentlichen Elementen des ambitionierten, von Österreich vorgelegten Resolutionsentwurfs Einigung erzielt werden, einschließlich zu einer Reihe konkreter Maßnahmen, um eine wirksamere Umsetzung von Schutzaufgaben durch FEO der VN zu gewährleisten (nähere Ausführungen siehe Kapitel III.1). Als besonders schwierig erwiesen sich in den Verhandlungen die Passagen zu Sanktionen, dem IStGH, der „Responsibility to Protect“ sowie der Informellen Expertengruppe des VN-SR zum Schutz der Zivilbevölkerung in bewaffneten Konflikten. In Unterstützung des österreichischen Vorsitzschwerpunkts sowie der Resolutionsverhandlungen fand am 5. November 2009 ein von Großbritannien organisiertes SR-Treffen im Arria-Format statt, in dessen Rahmen u.a. ehemalige Truppenkommandanten ihre Erfahrungen bei der Umsetzung von Schutzmandaten in FEO der VN präsentierten, um dem VN-SR zusätzliche Informationen über die konkreten Verhältnisse und Probleme beim Schutz von Zivilisten im Feld zu bieten.

Unter Vorsitzführung von Bundesminister Michael Spindelegger verabschiedete der VN-SR am 11. November 2009 im Rahmen einer hochrangig besetzten Debatte, in deren Verlauf über 60 Staaten das Wort ergriffen, einstimmig SR-Resolution 1894 (2009). Die Resolution wurde von 31 VN-Mitgliedstaaten miteingebracht und hat mittlerweile aufgrund ihrer substantiellen Inhalte Anerkennung als Meilenstein zum Schutz der Zivilbevölkerung in bewaffneten Konflikten gefunden. Österreich arbeitet seither mit Nachdruck an deren Umsetzung und wird seine intensiven Bemühungen auch nach Ende der SRMitgliedschaft fortsetzen. 


\section{EU-interne Zusammenarbeit und Stärkung der GASP}

Am 1. Dezember 2009 trat der Vertrag von Lissabon in Kraft. Durch ihn soll auch die Außenpolitik der EU gestärkt werden. Österreich unterstützt dieses Ziel und ist bestrebt, eine Stärkung der EU bei Internationalen Organisationen zu erreichen. Neben den beiden ständigen Mitgliedern im VN-SR aus den Reihen der EU, Frankreich und Großbritannien, war Österreich über die zwei Jahre seiner Mitgliedschaft das einzige nicht-ständige SR-Mitglied aus der EU. Österreich war es daher ein besonderes Anliegen, den Aktivitäten der EU im VN-SR entsprechendes Gehör zu verschaffen.

Österreich hat die gemeinsamen EU-Positionen als Basis seiner nationalen Positionen und Stellungnahmen im VN-SR berücksichtigt, beispielsweise Ratsschlussfolgerungen der EU-Außenminister zum Nahen Osten. Gleichzeitig hat Österreich im Rahmen der SR-Debatten konsequent auf die Leistungen der EU im Interesse der Friedenssicherung vor, während und nach Konflikten hingewiesen, wie etwa im Bereich der Sicherheitssektorreform sowie der Entwicklungs- und humanitären Hilfe. Eine große Bedeutung kam den militärischen Operationen und zivilen Missionen der EU in Konflikten auf der Tagesordnung des VN-SR zu, wie etwa der Überbrückungsoperation EUFOR Tchad/RCA zur Unterstützung der VN-Mission MINURCAT (bis März 2009), EULEX Kosovo zum Aufbau des Justiz-, Zoll- und Polizeisektors im Kosovo oder EUNAVFOR Somalia (Operation „Atalanta“) zur Bekämpfung der Piraterie vor der Küste Somalias.

Gleichzeitig galt es auch, das Profil von EU-HV Ashton im VN-SR zu stärken. Auf österreichische Initiative sprach EU-HV Ashton am 4. Mai 2010 erstmals im VN-SR. Zum Thema Zusammenarbeit zwischen den VN und Regionalorganisationen präsentierte sie im VN-SR die Beiträge der EU zur Wahrung des internationalen Friedens und der Sicherheit und betonte die Bedeutung einer starken Partnerschaft zwischen den VN und der EU zu diesem Zwecke.

Im Rahmen seiner Tätigkeit im VN-SR hat Österreich, wo immer möglich, auf der Basis etablierter Positionen der EU agiert und sich in dieser Hinsicht laufend mit der EU-Delegation in New York und auch mit den beiden EUPartnern im VN-SR, Frankreich und Großbritannien, abgestimmt. Beide Staaten haben jedoch erkennen lassen, dass ihre Position im VN-SR in vielen Fragen auch durch nationale Interessen bzw. durch ihre Sonderstellung als ständige Mitglieder bestimmt ist. Zu einem gemeinsamen Auftreten der Außenminister Österreichs, Frankreichs und Großbritanniens, Spindelegger, Kouchner und Miliband, kam es im Mai 2009 während der bewaffneten Auseinandersetzungen in Sri Lanka in der Frage des Schutzes der Zivilbevölkerung. Im Wege der Ständigen Vertretungen bei den VN in New York hat sich ein intensiver Koordinationsprozess entwickelt, in den neben den EU-Mitgliedern auch die anderen europäischen SR-Mitglieder Türkei, Kroatien 
(2009) sowie Bosnien und Herzegowina (2010) eingebunden waren. Über österreichische Initiative gelang es auch, den Leiter der EU-Delegation in New York nunmehr regelmäßig in diesen Koordinationsmechanismus einzubinden. Auf Ebene der Hauptstädte wurde dieser Mechanismus von halbjährlichen strategischen Treffen zu den wichtigsten bevorstehenden Themen im VN-SR zwischen den Zentralen in Paris, London und Wien begleitet. Gleichzeitig konnten österreichische Schwerpunkte in diesen Koordinationsprozessen mit Nachdruck verfolgt und umgesetzt werden.

Zudem sah es Österreich als seine Aufgabe, regelmäßig die anderen 24 EUMitgliedstaaten transparent und ausführlich über die Arbeit und Entwicklungen im VN-SR zu informieren. Auch im Politischen und Sicherheitspolitischen Komitee (PSK) in Brüssel hat sich Österreich um eine regelmäßige Information der EU-Partner über die Arbeit des VN-SR durch die im VN-SR vertretenen EU-Mitglieder bemüht.

Um die Fortsetzung der Bemühungen zur Stärkung der EU in den VN, sowie die Fortschritte bei österreichischen Schwerpunkten wie der Stärkung der Rechtsstaatlichkeit oder dem Schutz der Zivilbevölkerung nach Ende der österreichischen SR-Mitgliedschaft zu gewährleisten, hat sich Österreich um einen besonders engen Kontakt mit den EU-Nachfolgern im VN-SR, Deutschland und Portugal, bemüht, die in den kommenden Jahren diese Bereiche aktiv weiterverfolgen möchten. Österreich wird sich nunmehr darauf konzentrieren, in anderen VN-Institutionen (etwa im Falle einer Mitgliedschaft im Menschenrechtsrat) sowie bei anderen Internationalen Organisationen die Stimme der EU weltweit zu stärken. 


\section{Koordinationsprozesse und Arbeitsabläufe}

Die Mitgliedschaft im VN-SR stellte für die österreichische Diplomatie eine große Herausforderung dar, da der Umfang der Arbeit im VN-SR auch eine temporäre Anpassung der Strukturen im BMeiA erforderlich machte. Dies wurde durch eine interne Umschichtung von Personalressourcen ohne finanziellen Mehraufwand bewältigt. So konnten die ÖV New York, die die Hauptlast zu tragen hatte, sowie die Abteilung für Internationale Organisationen, die für die Koordination innerhalb des BMeiA verantwortlich war, für die Mitgliedschaft im VN-SR entsprechend verstärkt werden. Weitere Voraussetzung für eine erfolgreiche Bewältigung der SR-Mitgliedschaft war eine enge Kooperation mit dem gesamten Netz an Vertretungsbehörden und den Fachabteilungen des Hauses. Hier ging es vor allem um die Beschaffung von Informationen aus den Konfliktgebieten oder anderen SR-Mitgliedern sowie deren Analyse und entsprechende Einarbeitung in die österreichischen Positionen. Angesichts des Ausbaus der GASP war auch die Einbindung der Ständigen Vertretung Österreichs bei der Europäischen Union in Brüssel (ÖV Brüssel) zur Kohärenz des österreichischen Vorgehens in New York und Brüssel notwendig.

Im Rahmen der unmittelbaren Vorbereitungen auf die SR-Mitgliedschaft wurden ein Handbuch über die wesentlichen Arbeitsabläufe des VN-SR sowie eine Dossierliste über alle im VN-SR behandelten Themen samt den hiezu eingenommenen österreichischen Positionen erstellt. Auf dieser Grundlage wurden alle Vertretungsbehörden um eine intensivierte Berichterstattung zu den Themen des VN-SR ersucht. Zur besseren Einschätzung der Positionen der anderen SR-Mitglieder wurden die betroffenen österreichischen Vertretungsbehörden überdies angewiesen engen Kontakt zu den jeweiligen Außenministerien zu halten und laufend über die zu erwartenden Standpunkte ihrer Gastländer zu berichten.

Die personellen und inhaltlichen Vorbereitungsmaßnahmen mussten im Übrigen innerhalb sehr kurzer Zeit bewältigt werden, da zwischen der Wahl in den VN-SR und dem Beginn der Mitgliedschaft nicht einmal drei Monate lagen.

Die ÖV New York erstellte zu jedem einzelnen Tagesordnungspunkt im VN-SR, in den Sanktionenkomitees oder in den Arbeitsgruppen einen Vorschlag für eine österreichische Stellungnahme. Insgesamt ergingen in den zwei Jahren der österreichischen SR-Mitgliedschaft mehr als 4000 Berichte mit Informationen und ergänzenden Vorschlägen aus New York nach Wien. Jeder dieser Vorschläge wurde im BMeiA von den fachlich zuständigen Abteilungen geprüft und kommentiert. Sofern erforderlich, wie etwa bei Sanktionenfragen oder beim Thema Peacekeeping, erfolgte auch eine Koordination mit anderen österreichischen Stellen, insbesondere den Fachressorts (Bundesministerium für Finanzen, Bundesministerium für Inneres, Bundesministerium für Justiz, Bundesministerium für Landesverteidigung 
und Sport, Bundesministerium für Wirtschaft, Familie und Jugend) sowie mit anderen relevanten Stellen (z.B. Österreichische Nationalbank, Wirtschaftskammer Österreich). Auf Basis der Ergebnisse des Koordinierungsprozesses, für den meist nur wenige Stunden zur Verfügung standen, erfolgten die Weisungen an die ÖV New York. So wurden durch die Abteilung für Internationale Organisationen in den zwei Jahren insgesamt über 1.600 Weisungen zum VN-SR nach New York übermittelt.

Um die Planung und den Arbeitsprozess zu erleichtern, erstellte die ÖV New York im Laufe der zweijährigen SR-Mitgliedschaft überdies 104 „Wochenberichte“. Diese boten in Ergänzung zur täglichen Berichterstattung einen kompakten Rückblick auf die Aktivitäten des VN-SR in der jeweils vergangenen Woche, enthielten zugleich aber auch eine Vorschau auf die Folgewoche mit Informationen zu den anstehenden Themen und die zu erwartenden Positionen anderer SR-Mitglieder. Diese „Wochenberichte“ ergingen auch an alle betroffenen österreichischen Vertretungsbehörden, sodass diese über die Vorgänge im VN-SR laufend informiert waren.

Als Leitungsorgan für die österreichische Arbeit im VN-SR fungierte eine Lenkungsgruppe unter dem Vorsitz von Generalsekretär Johannes Kyrle. An dieser wirkte insbesondere auch der (für die Weisungsgebung in Fragen der österreichischen SR-Mitgliedschaft hauptverantwortliche) Politische Direktor des BMeiA mit. Diese Lenkungsgruppe erörterte im Rahmen wöchentlicher Videokonferenzen mit dem Ständigen Vertreter in New York - unter Zuschaltung des Vertreters Österreichs im PSK - die jeweils aktuellen SRThemen und die Grundlinien der von Österreich einzunehmenden Standpunkte. 


\section{Anhang}

\section{Zusammensetzung der österreichischen Delegation}

Botschafter Thomas Mayr-Harting, Ständiger Vertreter

Botschafter Christian Ebner, stellvertretender Ständiger Vertreter

Gesandter Nikolaus Lutterotti, Politischer Koordinator

Gesandter Konrad Bühler, Rechtsberater

Botschaftsrat Jürgen Heissel

Botschaftsrätin Gabriele Juen

Botschaftsrätin Ulrike Köhler

Botschaftsrätin Hannah Liko

Botschaftsrätin Ulrike Nguyen

Botschaftsrat Christoph Wieland

Botschaftssekretärin Katalin-Tünde Huber

Botschaftssekretärin Barbara Kaudel

Botschaftssekretärin Valerie Kyrle

Botschaftssekretärin Maria Holzmann

Presseattachée Verena Nowotny

Katja Wallner, Beraterin

Generalmajor Bernd Lubenik, Militärberater

Karl Deuretzbacher, beigeordneter Militärberater

\section{2. Österreichische Erklärungen im Sicherheitsrat und Ausgewählte Dokumente}

\subsection{Ausgewählte österreichische Wortmeldungen}

SC Debate - Respect for international humanitarian law

Statement by Ambassador Thomas Mayr-Harting, Permanent Representative of Austria to the United Nations

New York, 29 January 2009

Mr. President,

The Hague conventions of 1899 and 1907 contain the famous Martens Clause. It states that, in an armed conflict, civilians and belligerents remain under the protection of the law of nations that results "from the laws of humanity 
and the dictates of the public conscience". This is also the basic philosophy of the Four Geneva Conventions which are today universally recognized and have, in fact, become customary international law.

Nonetheless, we continue to witness serious violations of the obligations under IHL. I have found that this problem has confronted us on practically every issue that we have had to deal with since Austria joined the Security Council: from Gaza to Eastern Congo, Somalia and Darfur, but also in our debates on the protection of civilians and the future of UN peacekeeping.

We are therefore very grateful that, thanks to this timely initiative of the French presidency, we today have the very first chance to discuss this important issue in general terms, as a challenge that confronts the Security Council "horizontally" whenever it seeks to maintain international peace and security. We also thank the representatives of OCHA, OLA, OHCHR, UNHCR and ICRC for their briefings.

\section{Mr. President,}

The Austrian Report on the "Security Council and the Rule of Law" that we presented in April 2008 contained 17 recommendations on how the Security Council could support the strengthening of a rules-based international system. To my mind many of these are relevant for today's discussion. I would, nevertheless, like to focus on the following three core questions:

- What can the Council do to strengthen the universal legal framework of IHL?

- How can it help to prevent violations of and promote compliance with IHL?

- And what can it do to ensure accountability when IHL is violated?

Let me now turn to the first point: The Security Council and the strengthening of the universal legal framework of IHL:

We believe that, as one outcome of the debate we are beginning today, the Council should solemnly reaffirm the obligation of all parties to armed conflicts to fully respect IHL and to ensure accountability. In this context, it would of course be important that all States that have not yet done so accede to the Additional Protocols to the Geneva Conventions as well as other relevant instruments such as the Convention on Certain Conventional Weapons and its five existing Protocols, the Mine Ban Treaty and the Convention on Cluster Munitions, which contribute to the humanitarian goals of the Geneva Conventions. In our view the respect for IHL in Non-International Armed Conflicts is of particular importance, since they present the majority of present-day conflicts on our agenda. We commend and support the ongoing work of the ICRC to undertake extensive research which has resulted in a list of 161 customary rules of IHL. We can also support the proposal by the UN Legal Counsel to grant a protected status to UN sites. 
Second question: What can the Security Council do to promote compliance with the rules of IHL?

Promoting awareness about IHL and in particular proper training of armed forces is one way forward. This is an issue where the ICRC plays a crucial role. The Security Council should encourage States to intensify their national efforts in this field and to cooperate closely with the Red Cross in the training of their military personnel.

At the same time, the Security Council should make more systematic use of monitoring, reporting and early-warning mechanisms. The Council should insist that all reports it receives on the situation in specific conflict areas also cover the topic of IHL. The protection of civilians, in particular women and children, needs to be included systematically in mandates for UN peacekeeping missions, as well as in the work of Special Representatives, Special Envoys and mediators. The Security Council should have more frequent briefings by relevant authorities, including the High Commissioner for Human Rights and the Special Adviser on the Prevention of Genocide.

The Council should also aim at bringing together more closely the various strands of this debate. We have separate discussions on issues such as the Protection of Civilians, Children in Armed Conflict and Women, Peace and Security. We should look for greater synergies between these debates and draw some common conclusions. Let me give you one example: The establishment of the monitoring and reporting mechanism under Res. 1612 and the Working Group on Children and Armed Conflict. This mechanism has yielded tangible results in a number of areas. The Council should, therefore, once again consider expanding this model to other areas of major concern in the field of IHL, in particular in respect of sexual and gender based violence. We are looking forward to discussing this question again in conjunction with the forthcoming reports of the Secretary General on children and armed conflict and on sexual violence against women (SC-res. 1820).

This brings me to my third question: What can the Council do to ensure accountability and combat impunity when IHL is violated:

Whenever there are allegations of serious violations of IHL the first step ought to be an in-depth investigation, if possible by a neutral and independent body. The Security Council should therefore encourage States to make use of the fact-finding commission established in accordance with the First Additional Protocol. Alternatively, the Council may also consider establishing International Commissions of Inquiry, as it did in the cases of Darfur and Lebanon.

The primary responsibility to prosecute individuals who commit grave breaches of IHL of course lies with the States concerned. Whenever necessary, the Security Council needs to call upon such States to fully comply with their respective obligations and to take the appropriate national imple- 
mentation measures. This includes enacting the necessary penal legislation in accordance with the Geneva Conventions.

Furthermore, we need to recall the important role played by international criminal tribunals, and in particular the ICC, in promoting respect for IHL, by prosecuting and adjudicating perpetrators of genocide, crimes against humanity and war crimes. The Council should support criminal justice mechanisms and confirm its opposition to impunity. Where local institutions are unwilling or unable to prosecute perpetrators of international crimes, the Council should consider appropriate measures to encourage or compel accountability, including referral of a matter to the ICC.

In conclusion, let me stress that it is our hope - and we support the French Presidency in this regard - that a process can be started today to work towards greater compliance with the rules of IHL - and a more systematic and overarching approach of the Council in this matter. Austria stands ready to play an active role and to contribute to the work of the Security Council in this regard.

\section{SC - Debate on the situation in the Middle East, including the Palestinian question}

\section{Statement by H.E. Michael Spindelegger, Federal Minister for European and International Affairs of the Republic of Austria}

New York, 11 May 2009

\section{Mr. President,}

I would like to thank the Secretary General for his comprehensive briefing on the developments on the ground and Minister Lavrov for his initiative of bringing us together.

Four months ago, with the tragic violent confrontation in and around the Gaza Strip ongoing, we met here facing an unprecedented challenge to the Middle East Peace Process. The Security Council agreed on a resolution calling for an immediate and durable cease-fire and the withdrawal of Israeli forces from Gaza. Even though a fragile cease-fire is in place today, we saw no further progress at the political, security and economic levels. The parties and the international community are thus confronted with the urgent need of creating the conditions for re-launching a credible and comprehensive peace process.

Our discussion is taking place at an important moment for the future of the region: The new government of Israel is engaged in a strategic review of its approach towards its neighbours and the peace process. Palestinians have to face the challenge of bridging the deep internal rift, agree on unification and prepare for new elections in order to give new democratic legitimacy to their leadership. 
In this period of uncertainty and transition, the international community must shoulder its responsibility and engage with the parties in order to move towards substantial negotiations for a lasting settlement of the Middle East conflict. Therefore, the Security Council must reconfirm its steadfast support for the peace process and its basic parameters, namely durable peace and freedom from violence and terrorism based on a two-states-solution as required by the Road Map with two democratic States, Israel and Palestine, living side by side in peace within secure and recognized borders. I would like to thank the Russian Presidency for preparing a statement that will hopefully serve as a guideline to the parties for re-engaging in comprehensive negotiations on all tracks.

Mr. President,

Three months ago, at the Gaza Reconstruction Conference, the international community pledged its full support to humanitarian relief to the civilian population and to early reconstruction of the Gaza Strip. The Secretary General's report leaves no doubt that there is an unacceptable lack of progress in alleviating the dire living-conditions in the Gaza Strip.

While Austria supports coordinated action by all parties concerned to prevent the illegal flow of arms and ammunition into the Gaza Strip we urgently call on Israel to allow for the immediate and comprehensive supply of the civilian population of the Gaza Strip with goods to satisfy everyday needs and the requirements of reconstruction. The Gaza Strip needs fuel, cash and materials to repair schools, clinics, sanitation networks and shelters.

Together with EU partners Austria has contributed funds to be disbursed by the European Commission to socially vulnerable Palestinians in the Gaza Strip. Under the current circumstances, this money simply cannot be disbursed to those whom we want to support. Innocent citizens must not be held hostage by Israel because of irresponsible policies of an illegitimate political leadership in the Gaza Strip. Let me say this very clearly: The current policies imposed on the Gaza Strip deprive its people of their human dignity. This is not acceptable.

Rebuilding Gaza and moving forward on the path to peace also requires rebuilding trust, including by strengthening respect for the rule of law, human rights and international humanitarian law. In this context, I would like to thank the Secretary General for having provided us with a summary of the findings of the Board of Inquiry on a number of deeply disturbing incidents during the Gaza crisis. As a matter of principle, allegations of violations of international humanitarian law must be investigated wherever they occur and whoever may have committed them. Austria believes that this report also merits the further attention of this Council. 
Mr. President,

We are concerned about the lack of political progress in the West Bank, where Israeli road-blocks and closure policies continue to render regular social life and economic activities impossible. Settlement construction and the destruction of Palestinian homes in East-Jerusalem and in the West Bank undermine any meaningful peace talks and, in consequence, weaken the credibility of the political process and playing into the hands of radical forces.

At the same time, we urgently call on Palestinian political representatives to assume their responsibility and seriously engage in efforts to work towards a unified political leadership. In view of the upcoming round of talks in Cairo (16th / 17th May) we strongly encourage inter-Palestinian reconciliation behind President Mahmoud Abbas, which is key not only for the future of the peace process but also for the implementation of reconstruction efforts in Gaza. Palestinians need a government that enjoys legitimacy among their own population and is acceptable as a partner to Israel and the international community. Such a government needs to be fully committed to the two-states-solution and the other principles of the peace process.

In their efforts towards lasting peace in the region, the parties have the firm support of the international community, with the Security Council and the Middle East Quartet as its main actors. The international conference on the Middle East Peace Process that the Russian Federation plans to convene, in consultation with the Quartet and the parties, in Moscow in the course of this year will provide the international community with another important occasion to demonstrate this support.

Thank you

\section{SC - Debate on the implementation of Security Council Resolution 1820 (2008) \\ Statement by Ambassador Thomas Mayr-Harting, Permanent Represen- tative of Austria to the United Nations}

New York, 7 August 2009

I would first like to thank the United Kingdom presidency for organizing this debate. I would also like to welcome the Secretary-General. Austria applauds him for his unwavering commitment in the fight against all forms of violence against women.

The adoption of resolution 1820 (2008) a year ago was an historical event. The resolution clearly states that sexual violence against women in armed conflict can constitute a threat to international peace and security that requires the attention of the Security Council. Despite positive and successful steps undertaken by the United Nations and individual States, women conti- 
nue to fall victim to sexual violence used as a deliberate means of warfare. This takes place on a daily basis in conflict situations around the world.

Austria aligns itself with the statement to be delivered by the representative of Sweden on behalf of the European Union, as well as with that to be made by the representative of Costa Rica on behalf of the Human Security Network.

I would like to concentrate on a number of practical proposals on how the Council could further improve its action on this important matter. Systematic attention needs to be paid to the prevention of and protection from sexual violence in the daily deliberations of the Council. We welcome the recent adoption of resolution 1882 (2009), extending the scope of the monitoring and reporting mechanism to parties who perpetrate grave sexual violence against children in situations of armed conflict. As women and girls represent the majority of victims of sexual violence, there is a need to ensure that reporting is not strictly limited to victims under the age of 18. In this context, we would welcome further information in a follow-up report next year on an appropriate monitoring and accountability mechanism to be established by the Council. Austria also welcomes the work of the Council's informal Expert Group on the Protection of Civilians, which we see as a useful forum for discussing important protection concerns, including those in the fight against sexual violence. More consistent and comprehensive reporting on sexual violence in the Secretary-General's country-specific reports would enable the Council to address the protection of civilians, particularly women and children, from sexual violence in a more systematic manner. To this end, the Council should include specific reporting requirements in resolutions establishing or renewing mandates.

Briefings by the Secretary-General's special representatives, the Emergency Relief Coordinator, the High Commissioner for Human Rights and relevant rapporteurs will and can also provide the Council with information crucial to its work. We are fully aware of the sensitivities of collecting data from victims of sexual violence, who are often severely traumatized by the dramatic experiences they have gone through. Guidelines on information gathering must take ethical and safety concerns into account. Short- and long-term assistance for survivors of sexual violence, in particular accessible and quality health care, psychosocial support and legal advice should be a priority.

Let me also acknowledge the vital role that non-governmental organizations and humanitarian organizations play in this regard. Access constraints for humanitarian actors can severely affect the situation of victims of sexual violence. Today, a clear body of rules outlawing sexual violence, including in armed conflict, is contained in international human rights, humanitarian and international criminal law. In spite of this, perpetrators who systematically commit serious violations against women and girls still go largely unpunished. Allegations of sexual violence need to be thoroughly investigated and perpetrators need to be brought to account, including not only through prosecution, but also through the vetting of armed and security forces. This situ- 
ation demands further action by the Security Council to strengthen the rule of law and to end impunity. Where necessary, the Council should consider appropriate measures encouraging and ensuring accountability for those responsible for international crimes, including through the establishment of commissions of inquiry, referrals to the International Criminal Court and the imposition of targeted measures. Sanctions committees need to receive relevant information to this end, including through exchanges with other subsidiary bodies.

The Council took an important step when deciding to give priority to the protection of civilians in the mandate of the United Nations Organization Mission in the Democratic Republic of the Congo (MONUC). This led to the subsequent adoption of the Comprehensive Strategy on Combating Sexual Violence in the Democratic Republic of the Congo, endorsed by the Government. It is also a very important and positive development that the Government of the Democratic Republic of the Congo has now finally decided to take concrete measures in the five particularly dramatic cases to which the Council once again drew the attention of that country's authorities during its mission there. It is evidently necessary that the Council continue to follow up on this matter. We are encouraged by the valuable work of MONUC's new joint protection teams. We must draw on lessons learned by such best practices in order to transpose them to the contexts of other missions. That is certainly an issue with which we will have to deal when we consider, in general terms, the subject of the protection of civilians in peacekeeping.

The active contribution of women to peacebuilding and conflict prevention is crucial to achieving lasting peace. Resolutions 1325 (2000) and 1820 (2008) have underlined the need to include women in peacekeeping and peacebuilding measures. Further efforts are needed. The development of gender guidelines for military personnel by the Department of Peacekeeping Operations and the development of guidelines for mediators and their consistent implementation can contribute to improving our response to sexual violence in conflict settings. In order to effectively fight sexual violence, improved coordination throughout the system, as well as leadership and accountability, are needed. Austria fully supports the appointment of a senior person with responsibility to attend to the prevention of and response to sexual violence across the entire United Nations system.

Austria is grateful for the Secretary-General's willingness to provide the Council with annual reports on this very important matter, and we look forward to receiving them in the interest of enhancing the implementation of resolution 1820 (2008). 


\section{SC - High-Level Summit on nuclear nonproliferation and nuclear disarma- ment}

\section{Statement by H.E. Heinz Fischer - Federal President of the Republic of Aus- tria}

New York, 24 September 2009

Mr. President,

Mr. Secretary-General,

Excellencies, Ladies and Gentlemen,

This is an auspicious moment: Today the Security Council of the UN pronounces its commitment to a world without nuclear weapons.

For more than half a century, this goal has been pursued by concerned citizens and political leaders around the world. The international community has undertaken efforts to contain the threat but many have adjusted to it, almost accepting the nuclear shadow as part of life.

But any such complacency is ill-founded:

We have arrived at a fork in the road: We can maintain our course and hope nothing happens. Or we can seek real change.

Future historians will assess whether today is a turning point. This will not depend on the words spoken today but on the deeds that follow. In May 2010 the international community will convene at the NPT-Review Conference in New York. There, we will have to agree on measures that enable progress toward a nuclear weapon free world.

For Austria, the following points will be paramount:

1. The prospect of a world without nuclear weapons must become a goal shared by all States. Austria supports the idea of a Nuclear Weapons Convention equipped with a sophisticated verification mechanism. In the meantime, the NPT remains the core of the global nuclear disarmament and non-proliferation regime. But to fulfil its functions effectively, it must be strengthened, it must be institutionalized, and it must become universal.

2. The Nuclear-Weapons-States must reduce their arsenals. As you, Mr. President, noted in Prague, "Words must mean something". The words enshrined in Article VI of the NPT have to be taken seriously. There have been positive developments, yes, but Nuclear-Weapons-States must do more.

3. We must devise a process for entry into force of the Comprehensive Nuclear Test-Ban Treaty (CTBT). Austria and Costa Rica, as co-chairs of the Article XIV-Conference, worked hard to promote entry into force over the last two years. This will only be possible, however, with the political commitment of the 9 States that still need to ratify.

4. We must enable the Conference on Disarmament (CD) in Geneva to negotiate a Fissile Material Cut-Off-Treaty (FMCT). As current President of the 
Conference, Austria will make every effort to promote this goal. But to achieve progress the support of all its members is necessary.

5. Our capabilities to prevent nuclear materials from proliferating to States and non-state actors must improve. Security Council Resolution 15401 needs better implementation: Austria welcomes its comprehensive review next week. Most importantly, we must enhance the International Atomic Energy Agency's (IAEA) monitoring and verification capabilities and ensure strict export control for sensitive material and technologies. In view of the presence of Director General El Baradei here today, let me thank him warmly for his excellent work as head of the Agency over the last 12 years.

6. Finally, we must strengthen trust and confidence. Nuclear weapon free zones contribute significantly to sustainable stability; regions like the Middle East would benefit from such a regime. Ensuring that arms control mechanisms operate in a fair and transparent manner is crucial. Austria's proposal to multilateralize the nuclear fuel cycle under the control of the IAEA could help to avoid crises of trust such as those with regard to the Iranian and North Korean nuclear programs.

For clarification, I would like to underline that paragraph 11 of this Resolution refers to those countries that have decided to use nuclear energy for peaceful energy reasons, which is not the case for Austria.

Mr President,

Austria is very satisfied that the Resolution we just adopted is a strong text, a positive contribution to the ongoing disarmament process. But resolutions are not enough. Every State must accept responsibility and actively participate.

I promise: Austria's support will be there. You can also count on the European Union with its well-known position on these issues and its deep commitment to strengthening the multilateral system. And we can rely on civil society which throughout the years remained the motor behind disarmament efforts.

Mr President,

Today we identified our goals for the future. We now need the energy, the commitment and the persistence to move forward. The support by Heads of State and Government at this table today makes me confident that, together, we can reach our goal.

Thank you. 


\section{SC - Open Debate on the protection of civilians in armed conflict}

\section{National Statement by H.E. Michael Spindelegger, Federal Minister for European and International Affairs of the Republic of Austria}

New York, 11 November 2009

Mr. Secretary General,

Excellencies, Ladies and Gentlemen,

I will now speak in my national capacity.

At the outset, I would like to thank the Secretary-General and all other speakers for their presentations. The numerous challenges in the protection of civilians highlighted today show the urgent need to increase our efforts. The protection of civilians is central to the mandate of the United Nations and its peacekeeping missions. It is of utmost importance that mandates of the Security Council for the protection of civilians are effectively implemented by missions on the ground. This requires that the Council addresses the protection of civilians in its daily work in a systematic manner.

Let me now add a few additional points:

All parties to armed conflict must strictly comply with their obligations to protect civilians under international law. This applies to all parties in any conflict in the world, [be it in the Democratic Republic of the Congo, Darfur, Afghanistan or in Gaza], Austria has reaffirmed its position on several occasions that serious violations of international humanitarian and human rights law need to be thoroughly investigated. Accountability for serious crimes must be ensured by taking concrete measures to promote the rule of law, prevent future violations and seek sustainable peace, justice, truth and reconciliation. This requires action at the national and where necessary at the international level. The Security Council has an important role in ending impunity.

Austria is also greatly concerned about the detrimental effects of small arms and light weapons on civilians. They prolong conflicts, they endanger civilians and they undermine security, peace and stability. Parties to armed conflict need to take all required measures to protect the civilian populations from the effects of landmines and cluster munitions. Victims require enhanced protection and assistance.

Persons with disabilities are particularly affected by armed conflict and have so far not received adequate attention. The UN system has to address more effectively the protection and assistance needs of this vulnerable group.

It is clear that the primary responsibility to protect civilians rests with national governments. This is reaffirmed by the concept of the responsibility to protect civilian populations from war crimes, crimes against humanity and genocide. The UN Security Council as the UN organ with primary responsibility for the maintenance of international peace and security has an impor- 
tant role to play. The focus must be on saving lives through timely and decisive action taken at national, regional and international level.

We look forward to continuing this important debate on the protection of civilians and I would like to underscore Austria's full commitment to work with other Council members and all relevant stakeholders to implement resolution 1894 adopted today in order to ensure the protection of those who are most vulnerable in situations of armed conflict.

Thank you!

\section{SC - Debate on cooperation between the UN and regional and subregional organizations in maintaining international peace and security}

Statement by Ambassador Thomas Mayr-Harting, Permanent Representative of Austria to the United Nations

New York, 13 January 2010

It was with profound grief that we have learned of the terrible earthquake that struck Haiti last night. We are deeply moved by the tragic losses both among the Haitian people and the UN presence on the island. Let me extend our deepest condolences to the government and the people of Haiti and to the families of those who lost their lives in the service of the UN and the overall presence in Haiti. My Government is actively involved in emergency efforts within the EU and we are also considering how best to make a useful national contribution.

I would like to thank the Chinese Presidency for organizing today's debate and the PRST before us, which builds on the work started with resolution SC resolution 1631 (2005). Let me equally thank the Secretary-General and the representatives of the regional organizations for their statements. Austria aligns herself with the statement made by the delegation of the European Union to the United Nations.

The Security Council has repeatedly reaffirmed the role of regional and subregional organizations in the prevention, management, and resolution of conflicts in accordance with Chapter VIII of the Charter. In the light of the challenges of our century, it is clear that the UN cannot operate in isolation in maintaining international peace and security but needs to be complemented by regional organizations, with their understanding of conflicts and their root causes and their capacity to respond. In many cases regional ownership is key to the success of the peacekeeping efforts of the international community and active involvement of regional and subregional organizations is the means to ensure this regional ownership.

As pointed out by the Secretary-General, the challenges for effective cooperation lie in ensuring a clear division of labor and distribution of roles and 
tasks between the UN and regional organizations, and in enhancing regional organizations' capacities and ownership in the area of peace and security.

With its Common Security and Defence Policy, the European Union can make an important contribution in the field of peace and security and has developed significant capacities for conflict prevention and conflict resolution. The entry into force of the Treaty of Lisbon last month and the creation of the office of the High Representative for Foreign Affairs and Security Policy will allow the EU to speak with an even stronger and more unified voice to its partners. The EU's commitment to support the UN and the Security Council in its responses to threats to international peace and security is translated in the Union's support to all current UN peacekeeping operations. As the example of EUFOR Chad has shown, the EU can also play a useful bridging role in crisis theatres until the UN and/or regional organizations of the region in question are ready to take over.

Let me also commend the important work carried out by the OSCE under the Kazakh Chairmanship in Office, whose election observation and field missions operative in Participating States cooperate closely with UN agencies and missions. We are pleased that UN representatives will be invited to the address the OSCE Permanent Council in Vienna this year. We are also very pleased with the personal interest of the Secretary-General in the cooperation with the OSCE.

The Joint Declaration on EU-UN cooperation in Crisis Management signed in 2003, established a joint consultative mechanism and has proven to be a useful instrument for enhancing the partnership between the two organizations. Desk-to-desk consultations on conflict prevention between the UN and the EU could be increased and - as models of dialogue - be expanded to other organizations. In this context, we also welcome the Council's intention to hold informal interactive dialogues with regional and subregional organizations.

The challenges of today's UN peacekeeping operations, including the ever increasing demands for UN deployments, highlight the need for increased cooperation and capacity-building of regional organizations. Enhanced regional capabilities for peacekeeping and conflict prevention are a prerequisite for strengthening regional ownership and responsibility for crisis management. We commend the respective efforts already undertaken by regional and subregional organizations.

Austria fully supports a stronger role for regional and subregional actors such as the African Union, ECOWAS and SADC in conflict prevention and resolution. Since 2003, the EU has made a significant contribution, through the African Peace Facility, to the strengthening of the AU's ability to engage in peacekeeping operations on the African continent. We welcome the proposals contained in the Prodi report and in the subsequent report of the Secretary-General on support to AU peacekeeping operations as a valuable con- 
tribution to strengthening of the AU's peacekeeping capacities and to enhance the development of an effective UN-AU partnership.

Stand-by arrangements are of particular relevance in the area of peacebuilding. By linking or coordinating UN rosters of experts with those of regional organizations, such as the EU, we can increase the pool of available expertise. Furthermore, for the work of the Peacebuilding Commission, close collaboration with regional and sub-regional organizations is crucial. In addition, training activities need to be intensified to meet the rising demand for qualified civilian personnel in peace operations. The Austrian Study Center for Peace and Conflict Resolution has been conducting regular training programs for several years on civilian peacekeeping and peacebuilding. Many of its participants are now serving in missions of regional organizations, including the AU and the OSCE.

I would also like to point out the important role regional and subregional organizations are playing in implementing Security Council resolutions of a thematic nature. SC Resolution 1894 on the protection of civilians explicitly stresses the importance of consultation and cooperation between the UN and regional organizations to improve the protection of civilians in armed conflict. Consultation and Cooperation is also needed to improve women's participation in peace processes and to ensure the protection of children affected by armed conflict, as well as in combating impunity, corruption, terrorism and organized crime. All of these issues should form an integral part of the cooperation and capacity-building efforts between the UN and regional and subregional organizations, as well as among regional organizations themselves.

Finally, better exchange and sharing of best practices and lessons learned on all of the issues mentioned will be crucial, both between the UN and regional and subregional organizations as well as among regional organizations themselves. One of the lessons learned from the EU's civilian and military operations is that missions become more effective through the mainstreaming of human rights and gender aspects. Regional organizations will also be in a position to make a significant contribution to the successful preparations for the 10th anniversary of SC resolution 1325 later this year.

\section{SC - UNODC Briefing on threats to international peace and security Statement by Ambassador Thomas Mayr-Harting, Permanent Representa- tive of Austria to the United Nations}

New York, 24 February 2010

Let me start by thanking you for taking the initiative to organise this important briefing on drug trafficking and transnational organised crime as a threat to international peace and security. Austria attaches great importance to the ongoing fight against drug trafficking and organised crime. We also highly 


\section{Anhang}

appreciate the efforts of the delegation of France and support the Presidential Statement to be adopted today.

I would like to express my delegation's gratitude to the Secretary-General for his personal interest and commitment to this important issue. Let me also thank Undersecretary-General Costa for his valuable input and his committed leadership of the United Nations Office for Drugs and Crime. My country is honored to host important office in Vienna. This provides us with the opportunity to observe the remarkable work and that of its Executive Director on a daily basis. We appreciated the initiative by UNODC and France to present in Vienna a report on crime and instability in the run-up to today's briefing.

In various recent meetings of the Council, delegations - including my own underlined the need to develop a better understanding of the root causes and aggravating factors in situations of armed conflict or post conflict situations. We thank UNODC for contributing to our understanding, interlinking of data, combining illicit trafficking routes, conflicts and per capita income on international maps. All this data illustrates convincingly the link between drug trafficking and transnational organised crime, conflict and underdevelopment.

The globalization of transportation, communication and finance has also been to the benefit of illicit business and criminal groups, allowing them to organize themselves and operate transnationally. To put it more bluntly: The globalization of organized crime has so far moved much faster then the globalization of law enforcement. As a result crime is transforming from a threat at the personal and natural levels to a strategic menace that can affect international peace and security.

It is only by acting in concert that the international community will be able to prevail in the fight against drug trafficking and other forms of organized crime such as human trafficking, migrant smuggling or money laundering. We must help states to develop their own capacities, to rebuild and strengthen their institutions to administer justice and ensure the rule of law as well as to provide security. Here, too, UNODC can make a very important contributions, the Council should duly take into account when devising sustainable strategies for conflict areas.

Universal adherence to, and accurate implementation of, pertinent international legal instruments, such as the UN Convention against Transnational Organized Crime (UNTOC), the UN Convention against Corruption (UNCAC), the UN drug control conventions and the international conventions related to terrorism, should be our common goal. In the process of implementation, international human rights and standards of due process will have to be fully respected. Furthermore, we constantly need to improve the existing instruments, with a view to keeping pace with the changing nature of transnational crimes. Having agreed on a mechanism to monitor the implementation of UNCAC in Doha, the international community should now focus on a strong and effective review mechanism for UNTOC. 
The Council has repeatedly recognized the close connection between international terrorism and transnational organized crime in its various manifestations and underlined the need to enhance coordination of efforts at the national, regional and global levels. Here again UNODC can play an important role when delivering assistance. The Terrorism Prevention Branch also plays a crucial role in the Counter-Terrorism Implementation Task Force.

Recent studies have confirmed the relationship between corruption and organised crime, suggesting that corruption facilitates all forms of organised crime, which in turn ferments further corruption. The 7th Global Forum on Reinventing Government, held in Vienna in June 2007, underlined in the Vienna Declaration the need to increase transparency and accountability to combat corruption. More cooperation in the implementation of the United Nations Convention on Corruption will be needed, including the development of anti-corruption policies and institutions, as well as preventive anticorruption frameworks.

In this context, we would like to draw your attention to the upcoming establishment of the International Anti-corruption Academy (IACA) in Laxenburg, Austria. This Academy, based on the UN Convention against Corruption, aims at promoting anti-corruption measures through academic research and professional academic training. The main audience of the IACA will be inter alia law enforcement officers, judicial staff, private sector representatives and representatives from non-governmental and International Organizations. The establishment of the IACA will be another important step in the fight against corruption world-wide.

We fully support the invitation to the Secretary-General, in the PRST before us, to include information on transnational threats into his reports to the Council, when analyzing conflicts, assessing or planning missions and making recommendations for future action. More regular briefings by the Executive Director of the UNODC will further contribute to our understanding, and help the Council to integrate these issues into its work in a more consistent manner.

SC - Open Debate on the implementation of the note by the President of the Security Council (S/2006/507) ("Working Methods")

Statement by Ambassador Thomas Mayr-Harting, Permanent Representative of Austria to the United Nations

New York, 22 April 2010

I would first like to thank the Japanese Presidency for convening today's open debate. We are grateful to Japan for her chairmanship of the Informal Working Group of the Council. We also wish to say a special word of thanks to the S-5 for their important contributions to this debate. 
We believe that any effort to improve the working methods of the Council must focus on two goals: enhancing transparency and strengthening interaction between the Council and the UN membership at large. The Council must also aim at maximum transparency vis-à-vis the broader public, in particular through its cooperation with the media.

Over the last years, we have seen some improvement in the realization of these aims. The Council has increased the number of its open debates and briefings. The consultation process the Council has established with TCCs and PCCs before deciding on the mandate of a mission or its renewal is another step in the right direction.

In some instances, so-called "informal interactive dialogues" have provided the Security Council and interested countries with a useful framework for a direct, informal exchange on issues of concern to the Council. This is a path that we should explore further.

I should also like to commend the initiative by Uganda to organize an informal meeting with Member States on the occasion of the presentation of the last Annual Report of the Council to the General Assembly. We hope that this practice will be continued.

At the same time, there are other areas where we still see substantial potential for improving the Council's working methods. One such example is the cooperation between the Council and the Peacebuilding Commission

It has been our experience that chairpersons of country-specific configurations of the PBC contribute in a very useful manner to the public meetings of the Council on the country situations they deal with. At the same time, it has also been very helpful to listen to their additional comments during subsequent Council consultations, but so far this has of course only been possible when the chairperson also happened to represent a Council member.

We believe that it would be beneficial for the Council to have the chairs of all country-specific configurations of the PBC participate in the relevant consultations of the Council, independently of whether they represent Council members or not.

One important way to improve interaction between the Council and whole groups of the membership is an intensified cooperation with regional and subregional organizations. The Security Council debated this subject under the Presidency of China and agreed that an increasing interaction of this kind contributes towards a coherent and effective implementation of the Council's resolutions.

This is certainly true for the cooperation between the Council and the European Union which has a strong interest to contribute to the work of the Council in many areas of mutual concern, and even more so since the entry into force of the Lisbon Treaty. 
Austria has made a special effort to contribute towards increased transparency and interaction in the subsidiary Council bodies that we have the honour to chair.

In the Informal Working Group of the Security Council on International Tribunals, Austria is maintaining a close dialogue with the specially affected countries and the host countries of the Tribunals. We also organized an Arria formula meeting on residual issues of the Tribunals open to all UN Member States. We have introduced regular biannual briefings; we issued an annual summary of the activities of the Group and, for the first time, a chapter on the Working Group was included in the last Annual Report of the Security Council.

Furthermore, I would like to highlight some important developments in the Al-Qaida/Taliban sanctions committee, which Austria also chairs. Resolution 1904 of December 2009 substantially improves the procedures under the 1267 sanctions regime in terms of due process. For the first time ever, individuals and entities seeking a delisting will have the chance to present their cases to an independent and impartial Ombudsperson to be appointed by the Secretary General. It also directs the Ombudsperson to forward to petitioners all information about a negative decision provided by the Committee, including explanatory comments. SC resolution 1904 represents a significant step forward in improving the fairness and transparency of the 1267 sanctions regime, thus enhancing both its effectiveness and legitimacy. Still, much depends on the practical implementation of this resolution, especially on the appointment of an eminent Ombudsperson in the near future.

Transparency and interaction are not only constant challenges in the relations between the Council and the wider membership, but at times also within the Council itself.

With regard to some issues on the agenda of the Council, its decisions are prepared within various special formats. We are well aware of the political and practical reasons for which these formats have developed, but experience also shows how important inclusiveness and transparency can be for achieving and preserving the unity of the Council.

The Council's working methods have developed over the years; yet, they will remain work-in-progress. As the work of the Security Council adapts to changed circumstances, so should the Council's working methods. Involvement of the wider membership will remain key in order to serve the interests of the entire World Organization. 
SC - Debate on cooperation between the UN and regional and subregional organizations in maintaining international peace and security (HR Ashton) Statement by H.E. Michael Spindelegger, Federal Minister for European and International Affairs of the Republic of Austria

New York, 4 May 2010

Thank you Mr. President for organizing today's meeting, which gives me the great pleasure to welcome High Representative Catherine Ashton to her first briefing to the Council.

The entry into force of the Treaty of Lisbon in December brought about significant changes for the European Union, and especially for its foreign and security policy. With the strengthening of the office of the High Representative for Foreign Affairs and Security Policy, which will be supported by the European External Action Service, the European Union will be able to speak to its partners with a stronger and more unified voice. Among those partners, the United Nations occupy a preeminent place. As stipulated by the EU Treaty the EU's actions on the international scene shall be guided inter alia by the respect for the principles of the UN Charter.

The challenges we are facing in the 21st century - resolving and preventing conflicts, combating terrorism and organized crime, and preventing nuclear proliferation, to name but a few - clearly show that the UN cannot operate in isolation but require cooperation and concerted action by its international partners.

The Security Council has recently reaffirmed the important contribution of regional and subregional organizations to the peaceful settlement of local disputes and preventive diplomacy in the Presidential Statement adopted in January under the Chinese Presidency. Due to their understanding of particular conflicts and their respective root causes as well as their specific capabilities, regional organizations can have a comparative advantage for addressing crisis situations. In addition, local and also regional ownership are often key to the success of such efforts. The importance of active participation of civil society has to be highlighted in this respect.

In the field of international peace and security, the firm and well-established cooperation between the UN and the EU remains crucial. With its Common Security and Defence Policy, the European Union makes an important contribution and has developed significant capacities for conflict prevention and conflict resolution. The EU is currently engaged in 13 military operations and civilian missions and Austria contributes personnel to six of them. The EU's commitment to support the UN and the Security Council in its responses to threats to international peace and security is translated in the Union's support to all current UN peacekeeping operations.

Concrete examples of cooperation include the Horn of Africa, notably Somalia, where the EU is actively engaged in anti-Piracy efforts in order to protect 
shipments by the World Food Programme to the Somali population. Equally, the newly established EU training mission in Uganda - aimed at supporting the Somali transnational federal government by providing training to 2000 Somali security forces - is conducted in close coordination between the EU and the UN. Also in civilian crises management the EU is interested in a close cooperation with the UN and other international actors, as in Kosovo or Bosnia and Herzegovina, in Afghanistan, or in Guinea-Bissau.

When the Security Council debated the subject of cooperation of the UN with regional organizations in January, it agreed that an increased cooperation with regional organizations also contributes towards a coherent and effective implementation of Council resolutions by the wider UN membership. This is certainly true for the cooperation between the Council and the European Union which has a strong interest to add to the Council's work in many areas of mutual concern.

Here, as an example of the important role the EU is playing in translating Security Council resolutions into its own actions, I would like to point out two thematic issues: the protection of civilians in situations of armed conflict and women, peace and security. SC Resolution 1894 on the protection of civilians explicitly stresses the importance of consultation and cooperation between the UN and regional organizations to improve the protection of civilians in armed conflict. The EU has reiterated its firm commitment and active engagement to enhance the protection of civilians on various occasions. CSDP missions have included important protection concerns in their mandates, in particular on women and children affected by armed conflict. The protection of civilians was the main objective of EUFOR/Chad, aimed at improving the security of refugees and internally displaced persons. Civilian CSDP missions, such as EUMM Georgia and EUPOL DRC, also have an important monitoring and reporting role in this regard. The EU makes a particular contribution to sustainable peace with its expertise in the area of the rule of law and human rights. In this regard, I welcome ongoing efforts in the EU framework to further integrate the protection of civilians into CSDP missions and operations, taking into account best practice identified by the UN in this area.

One of the lessons learned from the EU's civilian and military operations is that missions become more effective through the mainstreaming of human rights and gender aspects, both in the planning and implementation phase. To this end, the EU has adopted various instruments relating to the implementation inter alia of SC Resolutions 1612 on children affected by armed conflict or 1325 on women peace and security in the context of our Common Security and Defence Policy. The EU is also actively supporting ongoing efforts to reinvigorate commitments on the 10th anniversary of Resolution 1325 in order to ensure effective implementation of this landmark Resolution. 
On all of these issues, better exchange and sharing of best practices and lessons learned is crucial, both between the UN and regional organizations as well as among regional organizations themselves.

Another important example of constructive EU engagement in multilateral negotiations is the Review Conference of the Treaty on the Non-Proliferation of Nuclear Weapons, which we have just opened. Nuclear disarmament, nonproliferation and ensuring a responsible development of peaceful uses of nuclear energy - in countries wishing to develop their capacities in this field - represent key challenges today to which the European Union has found a common response despite the large spectrum of views of its Member States. Thus also in the sensitive field of nuclear cooperation the European Union is making a substantive contribution.

The Lisbon Treaty has simplified the international representation of the European Union. The External Action Service will help to enhance its strategic direction, coherence and effectiveness and further improve the EU's cooperation with international partners, and in particular the United Nations. I hope that your visit to the Council today, Baroness Ashton, will be the first in a series of regular appearances in this body.

\section{SC - Urgent meeting on the situation in the Middle East, including the Pales- tinian question}

\section{Statement by Ambassador Thomas Mayr-Harting, Permanent Representa- tive of Austria to the United Nations}

New York, 31 May 2010

Thank you for convening this meeting so rapidly. I would like to welcome the presence of the Foreign Minister of Turkey, His Excellency Ahmed Davutoglu. I would also like to thank the Deputy Secretary-General for her presence and ASG Fernandez-Taranco for his briefing on the Israeli military operation against the flotilla sailing to Gaza.

Austria is deeply shocked at the loss of lives and casualties and wishes to express her condolences to the families of the victims. We are very disturbed about this dramatic escalation and condemn the use of violence that has led to a high number of victims. Austria's Foreign Minister Spindelegger has called for a rapid, independent and full investigation of this attack which occurred in international waters. We have also formally communicated this position to the Ambassador of Israel in Austria. Moreover, we call on the Israeli authorities to urgently provide the concerned states with consular access to and information about their citizens detained in Israel.

The lack of full compliance with Security Council resolution 1860 is at the core of the deplorable events of last night. The humanitarian situation in Gaza remains a source of utmost concern. 
Austria has consistently acknowledged Israel's right to protect her citizens against indiscriminate attacks emanating from Gaza. We repeat our call for an immediate end to all such attacks. At the same time, we believe that these concerns cannot in any way justify what has happened today. We regard the ongoing blockade as totally unacceptable and counterproductive. It only benefits spoilers and advocates of violence.

We reiterate our call for an immediate, sustained and unconditional opening of all crossings for the flow of humanitarian aid, commercial goods and persons to and from Gaza.

We urge Israel to take immediate and credible steps to avoid any reoccurrence of such violent incidents in the future and to act in full accordance with her obligations under international law and international humanitarian law. We call on all sides to exercise utmost restraint and refrain from any action that could prolong or even worsen the plight of the civilian population of Gaza.

\section{SC - Open Debate on children and armed conflict}

Statement by Ambassador Thomas Mayr-Harting, Permanent Representative of Austria to the United Nations

New York, 16 June 2010

\section{Madame President,}

At the outset, I wish to welcome you, Madame President, and I also would like to thank Ambassador Heller and the Mission team for their excellent work in preparation of this important debate.

Austria aligns herself with the statement to be made later by the European Union. As a Member of the Human Security Network, as well as of the Group of Friends on Children and Armed Conflict, Austria also supports the joint statement of the Network to be delivered by Costa Rica and the first ever joint statement of the Group of Friends to be delivered by Canada.

I wish to thank SRSG Coomaraswamy, Deputy Executive Director Johnson and ASG Khare for their presentations. We highly appreciate the work of the SRSG and all those who contribute to implementing the children and armed conflict agenda within the Secretariat and in UNICEF. My delegation and I personally also wish to extend a warm welcome to Ms. Manju Gurung from Nepal. We thank her for having shared her thoughts and her very moving experiences, which serve as a stark reminder of why we are here today. We welcome the signing of an action plan by the parties in Nepal and the swift completion of the discharge process. The successful mission of SRSG Coomaraswamy to Nepal last December underlines the importance of her field visits for an effective follow-up on the recommendations of the Security Council and its Working Group. 
Progress has been made in the past year also in other parts of the world, as set out in the Secretary-General's most recent annual report. We welcome the signing of action plans to halt and prevent the recruitment and use of children with parties in the Philippines and the Sudan, as well as the positive developments in Burundi, which allowed for the de-listing of the armed groups formerly listed under that country situation.

At the same time, the fact that parties to armed conflict have continued to commit grave violations and abuses against children in conflict situations around the world is deeply worrying. We take note of new listings of parties that recruit and use children and engage in patterns of killing and maiming or sexual violence. We call on all parties listed in the annexes immediately to halt those violations and to prepare and implement concrete, time-bound action plans to ensure the effective protection of children. We agree with the Secretary-General that direct contact between the United Nations country teams and non-State actors is important in this context. We therefore support his call on Member States to allow for such contacts in the framework of Resolutions 1539, 1612 and 1882.

The reported increase in attacks against schools, as well as teachers and students, and the specific targeting of girl students in certain country situations, is a deeply worrying development. We strongly condemn such heinous acts, which violate international humanitarian law and deprive the children of any meaningful prospects for a better future.

We wish to acknowledge the detailed information provided in the SecretaryGeneral's report on the criteria and procedures used for listing and de-listing parties to armed conflict as well as the ongoing development of templates for action plans to address and prevent patterns of killing and maiming and/or rape and other sexual violence against children. We agree with the SecretaryGeneral's understanding of the notion of a "pattern", which involves a "multiple commission of acts being perpetrated in the same context", but also wish to stress that this from our point of view should not necessarily imply a high quantitative threshold.

With the introduction of new listing criteria in Resolution 1882 the provision of timely, accurate, reliable and verifiable information to the Council, and thus an increased monitoring and reporting capacity of the UN system, has become even more important. We call, therefore, on all relevant UN entities to cooperate and coordinate in order to create synergies and to allow for the systematic exchange of information. We also believe that in certain situations - such as the case of the Lord's Resistance Army - regional cooperation among UN actors in the field needs to be strengthened.

The report of the Secretary-General rightly draws our attention to those parties to conflict that continue to commit crimes against children, despite repeated calls of the Council. The Council must be determined to ensure 
respect for its resolutions and needs to be prepared to take vigorous measures against persistent perpetrators.

In order to increase the coherence of the Council's work, provisions pertaining to violations of international law committed against children should be included in the mandates of the relevant sanctions committees. Interaction of the SRSG and the Working Group with sanctions committees and their expert groups should be enhanced. The recent briefing by SRSG Coomaraswamy to the DRC Sanctions Committee, which included provision of specific information on individuals recruiting and using children, was an important first step. In this context we also support the Secretary General's recommendation to incorporate child protection expertise in sanctions committees' expert groups. At the same time, the Council must also consider specific recommendations from its Working Group on parties committing violations and abuses against children, in order to take direct action in particularly serious situations, where no sanctions committee is in place.

We welcome the fact that the PRST to be adopted at the end of this debate addresses these issues in a very forward-looking manner.

In closing, let me express my country's sincere appreciation for the excellent work that has been carried out by the Mexican Chair of the Working Group over the past one-and-a- half years. The Group has been doing important work in developing concrete recommendations on various situations on the basis of country-specific reports of the Secretary-General and exchanges with the Member States concerned. In view of the considerable workload, as well as the urgent need to build up a historical memory, Austria strongly supports the repeated request to the Secretary-General to provide administrative support to the Group. This could also enable the Group to further improve its working methods and the follow-up of its conclusions and recommendations as well as to proceed to a more effective use of its toolkit, in particular through the timely realization of a field visit.

\section{SC - Open Debate on the promotion and strengthening of the rule of law in the maintenance of international peace and security}

\section{Statement by Ambassador Thomas Mayr-Harting, Permanent Representa- tive of Austria to the United Nations}

New York, 29 June 2010

At the outset, let me congratulate the Mexican Presidency for organizing today's open debate on the subject "The promotion and strengthening of the rule of law in the maintenance of international peace and security" and for preparing the concept note (S/2010/322). Let me add that in view of your outstanding experience in this matter, it is a particular pleasure to have you, Mr. President, presiding over the Council here today. We warmly welcome 
the initiative. I would also want to thank DSG Asha-Rose Migiro and USG Patricia O'Brien for their statements and their presence today.

Austria would like to reaffirm its firm commitment to an international order based on international law, including human rights law, and the rule of law with the United Nations at its core. We believe that international law and the rule of law are the foundations of the international system. Clear and foreseeable rules, respect for and adherence to these rules, and an effective multilateral system to prevent or sanction violations are preconditions for lasting international peace and security. In our view, it is imperative to strengthen the rule of law in all its dimensions, i.e. at the national, international and institutional levels.

For many years, Austria has placed a particular focus on the role of the Security Council in strengthening a rules-based international system. You, Mr. President, have already kindly referred to our initiative that we started in 2004 on "The Rule of Law and the Security Council". We started this together with the NYU School of Law and launched a series of panel discussions on this topic and, together with Mexico, Liechtenstein and other like-minded members of the Group of "Friends of the Rule of Law", prepared a Final Report. This was presented in New York in April 2008 and also published as a Security Council document (S/2008/270). The Report contains 17 specific recommendations how the Security Council could strengthen the rule of law in its various fields of activity. During our membership on the Council we have consistently worked with other delegations in order to implement and mainstream these recommendations in the Council's daily business.

In this context, Austria also would like to commends the efforts of the SC Informal Working Group on Documentation and Procedural Questions in its efforts to strengthen the transparency of the Council's working methods by reviewing and updating the Presidential Note S/2006/507 and, thereby, enhancing the rule of law in the everyday work of the Council.

Since the last open debate on the rule of law in June 2006 significant progress has been made with the establishment of the Rule of Law Coordination and Resource Group, chaired by the DSG and supported by the Rule of Law Unit. We are very grateful for the personal involvement of the DSG in this important matter. We strongly support the Group and the Unit in their efforts to ensure a coordinated and coherent response and we also support the specific proposals made by the DSG on this subject.

Among the topics raised in the concept paper, let me first address the importance of the rule of law in conflict and post-conflict situations. In resolution 1894 (2009), the Security Council reaffirmed that the deliberate targeting of civilians and the commission of systematic, flagrant and widespread violations of applicable international humanitarian and human rights law in situations of armed conflict may constitute a threat to international peace and security and imply appropriate measures by the Council. Respect for interna- 
tional humanitarian law by all parties to conflict is essential for the protection of civilians and should be an important aspect of any comprehensive strategy to resolve conflict. We underline the special rights and protection of women and children under international law, which we urge all parties to conflict to respect.

The rule of law is the cornerstone of all peacebuilding efforts. We call on the Council to express its commitment to ensure that all UN efforts to restore peace and security themselves respect the rule of law. The promotion of the rule of law in post-conflict situations can only be achieved through an integrated and coordinated approach, which encompasses all actors involved. In this context, the Peacebuilding Commission has a vital role to play. We commend the important contributions by international and regional organizations in this area, including by the European Union and the International Development Law Organization.

Turning now to international justice and the peaceful settlement of disputes, Austria strongly supports the role of the International Court of Justice as the principal judicial organ of the United Nations and calls on all States that have not yet done so to consider accepting the jurisdiction of the Court in accordance with its Statute. For its part, Austria accepted the compulsory jurisdiction of the Court four decades ago.

Regarding the international efforts to end impunity for the most serious crimes of international concern, Austria stresses the important role of the International Criminal Court, ad hoc and mixed tribunals, as well as the specialized chambers in national tribunals. Austria strongly believes that the permanent ICC is one of the most effective tools to buttress the rule of law and combat impunity. In this regard, we commend the stocktaking of international criminal justice undertaken by the First Review Conference of the ICC, held in Kampala upon the invitation of the government of Uganda from 31 May to 11 June 2010. While the ad hoc Tribunals for the former Yugoslavia and Rwanda are only temporary institutions, Austria is working as chair of the SC Informal Working Group on International Tribunals to establish a residual mechanism to take over their residual functions and thus preserve justice and the rule of law.

We believe that the UN and the Security Council must continue to be at the forefront of the fight against impunity and ensure that alleged violations are investigated and those responsible for crimes are held accountable. Accountability must be ensured by taking measures at the national level, such as through domestic prosecutions, truth-seeking, providing reparations for victims and through institutional reform. Justice is essential to achieve lasting peace and reconciliation, as well as to avoid the recurrence of future violations.

As chair of the Al-Qaida/Taliban sanctions committee, Austria is also committed to enhancing the efficiency and credibility of sanctions regimes. Sanc- 
tions play an important role in promoting compliance with international law and fighting international terrorism. However, when targeting individuals, sanctions also raise questions regarding procedural guarantees and due process. Austria welcomes the adoption of resolutions 1822 (2008) and 1904 (2009), which provide for substantial improvements of the listing and delisting procedures of the Al-Qaida/Taliban sanctions regime. As others, we warmly welcome the recent appointment of Ms. Kimberly Prost, an outstanding lawyer, as Ombudsperson by the Secretary General and I would specifically like to assure USG O'Brien who made the point that the committee is looking forward to working with her very closely.

Finally, my delegation would like to express its strong support for the Presidential Statement which the Council will adopt later today and the request to the Secretary-General to prepare a follow-up report to his 2004 report on "The rule of law and transitional justice in conflict and post-conflict societies."

Thank you, Mr. President.

\section{SC - Summit on ensuring the Security Council's effective role in maintai- ning international peace and security}

Statement by H.E. Heinz Fischer, Federal President of the Republic of Austria

New York, 23 September 2010

Emerging from the biggest catastrophe in the 20th century, a war in which all together 50 million people lost their lives, the United Nations were founded to save succeeding generations from the scourge of war. The Security Council was entrusted with the responsibility to maintain international peace and security. Over the last 65 years the Council has made an enormous contribution to this noble goal. For those affected by armed conflict this often is a question of life and death, of security and development and of respect for the rule of law and human rights. No continent was spared, and while in the early years the Council had to deal primarily with interstate conflicts, the nature of conflict has changed significantly in recent times. Intra-state conflicts involving non-state actors as well as transnational threats such as terrorism, organised crime, drug trafficking, proliferation of weapons of mass destruction etc. have created new challenges for the United Nations.

Peacekeeping - originally not foreseen in the UN-Charter - has developed into a particularly successful crisis management tool. Over the last decades, the character of peacekeeping has changed: after monitoring of ceasefires and interpositioning, peacekeepers of today find themselves in the midst of conflicts facing complex tasks and challenges. Recognising the importance of UN peacekeeping, Austria started contributing troops, police and experts to 
UN-operations immediately after joining the United Nations. 90.000 Austrians in the service of peace since 1960 are proof of our unwavering commitment to the maintenance of international peace and security.

Our willingness to assume global responsibility furthermore manifests itself in our third membership in the Security Council. Confronted with today's threats, we identified the protection of civilians in armed conflict as a key priority. The effective protection of the civilian population is the precondition for sustainable peace, and as long as UN peacekeeping missions are involved, it is also a question of the credibility of the Council.

Resolution 1894 provides a solid basis for further enhancing the protection of the most vulnerable. Creating a favorable protection environment goes beyond the protection from physical violence. It must be complemented by activities in the fields of the Rule of Law, human rights, Security Sector Reform, Disarmament, Demobilization and Reintegration and the empowerment of local society. The swift implementation of resolution 1894 will therefore make an important contribution to the Council's effectiveness in this core area.

An equally important topic is the role of women in peace and security. We must seize the unique opportunity of the 10th anniversary of resolution 1325 this October to renew our commitment and work towards the increased participation, representation and full involvement of women in preventive diplomacy, mediation, peacekeeping and peace-building. In this context I would like to commend Secretary General Ban Ki-moon for his strong leadership and encourage him to continue to promote the cause of women.

These efforts must be accompanied by an increased protection of women and girls, especially from sexual violence. The Security Council has several times reaffirmed its role in fighting impunity for such violations. Let me also thank Uganda (as Presidency of the Council for the month of October) for their commitment to further advance the implementation of this landmark resolution.

The systematic consideration of the "protection of civilians in armed conflict", "children and armed conflict" and "woman peace and security" demonstrates the ability of the Council to respond effectively to today's challenges.

In order to sustainably resolve a conflict, the Council should pursue an integrated approach throughout all stages of conflict. Therefore peacekeeping and peace-building need to go hand in hand to provide security and development with the clear aim of strengthening national institutions and preventing a relapse into conflict.

Finally I would like to emphasise, that prevention is the most effective and efficient way of managing conflicts. We therefore highly welcome the efforts by the Secretary General to strengthen the UN's capacity in early warning and conflict prevention and to continue his good offices and mediation acti- 
vities. As a member of the European Union we strongly believe in the partnership between the United Nations and regional organisations in this regard.

In conclusion allow me to congratulate you on organising this summit and preparing a Presidential Statement that enjoys our full support. Let me close by reiterating that the Security Council will exercise its role in a most effective and credible manner, when the guiding principle of its actions is the rule of law - clear and foreseeable rules equally applicable to everybody.

\section{SC - Open Debate on women and peace and security}

\section{Statement by H.E. Michael Spindelegger, Federal Minister for European and International Affairs of the Republic of Austria}

New York, 26 October 2010

Mr. President, I would like to thank you for the initiative to convene this high-level open debate on Women and Peace and Security, marking the 10th anniversary of the adoption of resolution 1325 (2000). Your presence here shows the support of Uganda to the issue. Let me further congratulate you for the efforts that resulted in the Presidential Statement before the Council today. I would also like to thank the Secretary-General for joining us from far away, which is testimony to his personal commitment to this subject.

It is a particular pleasure to welcome Under-Secretary-General Ms. Michelle Bachelet in the Chamber. I would like to congratulate her on her appointment and assure her of Austria's full support. We are convinced that UN Women will play a central role in further advancing this agenda. We are equally grateful for the valuable input of Ms. Thelma Awori representing the Civil Society Advisory Group on Women, Peace and Security. Civil society has always been a driving force behind this issue and we are looking forward to further advancing this agenda in close partnership.

When adopting this landmark resolution 10 years ago, the Security Council recognized that equal participation, representation and full involvement of women in all aspects of peace-building and security, the protection of women as a group with specific needs and concerns as well as the prevention of sexual and other violence are not only a security issue but also vital for sustainable peace and stability. With the adoption of the resolutions on "sexual violence" and "women and girls in post conflict situations" the Council now disposes of a strong and well developed normative framework.

Over the past decade, progress in the implementation has been slow and uneven. The resolution's real impact remains to be felt on the ground in many areas. All too often women do not make it to the tables where decisions are taken in peace processes or post-conflict reconstruction that have a direct impact on their lives. There are no issues that are not also women's issues. Every month hundreds of women and children fall victims to sexual vio- 
lence under the eyes of their governments and the international community. Women and girls with disabilities remain even more vulnerable. Ten years on, our focus must therefore lie on how we can ensure better and more coherent implementation of the objectives enshrined in these resolutions and make a real difference for women in conflict and post-conflict situations.

The Council has at its disposal a whole range of tools for the implementation of resolution 1325. These include measures such as mandates of peacekeeping and other relevant missions, briefings and reports, commissions of inquiry as well as targeted measures and sanctions. We have to be ready to use these tools and to translate words into practice in a consistent manner. We have to ensure that those that disregard the Council's decisions are being held accountable.

The Arria Meeting of the Security Council on 19 October co-chaired by Austria, Mexico and the United Kingdom has made it clear that we need more information on progress and on what measures have been successful. The comprehensive set of indicators on 1325 which received the Council's support today, will finally close this important gap and provide us with qualitative and quantitative data on important areas such as the prevalence of sexual violence or the number of women participating in peace-processes. This information will allow us to better guide and target our actions in the future. We hope that the indicators will now become operational as a matter of urgency. We do believe that all Member States should use the indicators relevant for their country's situation, including in their national action plans in order to receive a truly global picture of the implementation of resolution 1325.

As a next step we request the Secretary-General to include the information gathered on the basis of the indicators in his country-specific and relevant thematic reports in a systematic and comprehensive manner. Without accurate and timely information, it is difficult for the Council to take appropriate action in areas that need our urgent attention, such as the prevention of sexual violence. We hope the Council will in the future also receive briefings on situations, where data gathered through the indicators suggest an outbreak of violence against women or a further deterioration of a situation. Early warning and prevention is still by far the best protection.

"Women, Peace and Security" needs to be on the agenda of the Council every time the Council considers a country situation or relevant thematic issues. The establishment of an Informal Expert Group would have further enabled the Council to evaluate information on the situation of women and girls in conflict situations around the globe. We hope that the Council will revert to this question in the near future, once the indicators are fully operational. The Security Council has to show active leadership and shoulder its responsibility for the implementation of its resolution 1325. 
The high level event on resolution 1325 organized by Canada in the margins of the general debate already provided us with an opportunity to make concrete and measurable commitments to implement resolution 1325. On this occasion I have already announced a few Austrian contributions. Today, I would like to add further contributions.

At the national level:

* The development and implementation of National Action Plans is crucial to improve the implementation of Res. 1325. Austria was one of the first countries to develop a National Action Plan for resolution 1325. We will take the 10th anniversary of Resolution 1325 as an opportunity to revise our National Action Plan. Work will start beginning of 2011 in close cooperation with civil society and other line ministries.

* Austria is committed to a continuous enhancement of its rule of law standards. The Rome Statute is the first international treaty to classify crimes against women, like rape or other forms of sexual violence, as crimes against humanity, war crimes or genocide. Austria is currently in the process of incorporating the crimes of the ICC Statute into its criminal code.

* Austria commits to contextualize education, and pre-deployment training of its armed forces personnel in order to address specific operational realities in regions of deployment, including the impact of conflict on gender relations and the role and participation of women (on the basis of relevant UN guidelines);

At the international level:

Supporting the UN in the implementation of 1325

* The newly-established entity "UN Women" has a central role in coordinating the UN's activities in implementing Res. 1325. Austria is committed to support UN Women and its efforts in making the newly developed indicators operational. In this context, Austria will provide UN Women with voluntary financial contributions.

Supporting the implementation of 1325 through the Austrian multilateral development cooperation

* Through the Austrian Development Agency, Austria supports and implements projects tailored towards the implementation of 1325, in particular in relation to violence against women, DDR, cooperation with civil society for conflict prevention and peace-keeping. We will continue with these efforts. The Austrian multilateral development cooperation will keep a strong focus on women and children in crisis and post conflict situations.

* With its expertise in the development of a National Action Plan on 1325, Austria stands ready to work together in the context of a 'Twinning Project' with partner countries to support the establishment of National Action Plans on 1325. 
Supporting the implementation of 1325 through Austria's engagements in the area of peacekeeping

* Austria commits to deploy gender experts (e.g. Military Gender Advisor) to military components of peace operations if designated and posted by the international community.

* Furthermore, Austria undertakes to ensuring the availability of immediate-response personnel (Gender Field Advisor, Gender Focal Points) within national contingents to address women and girls' urgent needs, with particular respect to health, security and justice.

* Austria will continue to further strengthen its training activities on 1325, in particular in pre-deployment trainings for peace and humanitarian operations that are being provided for civilian and military experts from around the world by the Austrian Study Center for Peace and Conflict Resolution (ASPR), and increase its training efforts in the Austrian Diplomatic Academy in 2011.

* Including through its Special Envoy for International Women's Issues, Ambassador Dr. Ursula Plassnik, Austria will continue to promote the implementation of Resolution 1325 including in the UN, the EU, the Council of Europe and the OSCE. Austria is committed to dialogue initiatives promoting in particular gender equality and strengthening women in public life and dialogue activities. For example, Austria has established an international Network on "Promoting Female Leadership in Intercultural and Interreligious Dialogue" first meeting in June 2010. Certainly, I also commit to raise awareness in my bilateral contacts for Res. 1325, where appropriate.

Let me finally reiterate my hope that the commitments to action will not be a onetime effort limited to the 10 year anniversary. We are ready to follow-up on our commitments and to review progress in the implementation annually at the Open Debates on women, peace and security.

\section{SC - Debate on Sudan}

Statement by H.E. Michael Spindelegger, Federal Minister for European and International Affairs of the Republic of Austria

New York, 16 November 2010

I would like to thank Secretary-General Ban Ki-moon and President Thabo Mbeki for all their efforts and for briefing us today. I also listened with keen interest to the statements by the Foreign Minister of the Sudan as well as the Secretary-General from SPLM. I thank Foreign Secretary William Hague for his initiative, which gives the Security Council an opportunity to issue a strong message of support for sustainable peace in the Sudan, and in particular the implementation of the CPA. 
In the past few months we have witnessed some important positive developments. Let me mention only two: the elections held in the Sudan and the marked improvement of relations between the Sudan and Chad.

Although the implementation of the CPA has been laborious, much has been accomplished. We trust that both parties will fulfill their commitments to implement the CPA, in particular to undertake credible referenda and to abide by their outcome. An EU-observer team is deployed in Sudan this week.

Last week, in a conference that took place in Vienna, high-level participants from the North and the South demonstrated their consensus on one core point: Whatever the outcome of the referenda, the North and the South will have to strive for a mutually beneficial coexistence.

We all know what is at stake and what arrangements still have to be agreed upon. We welcome that progress in the preparations for the Referenda has been achieved. Still, further progress is needed, in particular in the preparation for the Referendum in Abyei. Preparations also have to take place for the Popular Consultations in the Blue Nile and Southern Kordofan States. The parties also need to prepare in a timely manner for the post-referendum phase. In that sense, we welcome the signing of a Framework Agreement in Khartoum.

Strengthening the rule of law, ending impunity and ensuring human rights for all will be important in this process in order to guarantee lasting peace and stability. The full inclusion of women in these processes is also of utmost importance.

Austria has offered technical expertise in the area of citizenship, and upon the invitation of the parties, has discussed this offer with interlocutors in the North and the South in October. We are ready to continue working with both sides in a transparent and open manner.

We must work to avert conflict, to ensure regional stability and to protect civilians. Therefore, contingency planning by UNMIS is important. We welcome the implementation of the Comprehensive Strategy on the Protection of Civilians. Increased patrolling, especially long range patrols, and an extended UNMIS presence in remote areas and potential hotspots is very important. Freedom of movement is crucial for UNMIS to fulfill its tasks. We also believe that the participation of UNMIS in the regional strategy for the protection of civilians from attacks by the LRA is essential.

We are deeply concerned about continued insecurity in Darfur and condemn the abduction of peacekeepers, humanitarian workers and demand their immediate release. We also condemn the violence against civilians in and around IDP camps. We urge all parties to allow free access to everyone in need of humanitarian assistance. We are also concerned about the recent arrest and detention of human rights activists and journalists. 
UNAMID has an important role to play in protecting civilians and needs the full support of the Government of the Sudan and all other parties involved. The safe and voluntary return of the high number of IDPs in Darfur must be ensured. The New Strategy on Darfur by the Government of the Sudan and its people-centred approach is a welcome step; however, we need all parties to engage fully and constructively in the peace process without preconditions. Also in this context, I would like to highlight the importance of the full participation of women in the peace negotiations on Darfur in line with resolution 1325, as well as an adequate participation of civil society.

Austria strongly supports the work of the African Union/UN Joint Chief Mediator Djibril Bassolé and the facilitation of the African Union High Level Panel under the leadership of President Thabo Mbeki. We strongly support all efforts to strengthen mechanisms to promote justice, accountability and reconciliation, in particular the International Criminal Court. Peace and justice must go hand in hand.

Mr. President, the primary responsibility to act lies with both partners of the Government of National Unity of the Sudan. Enduring co-operation between the parties will be essential for the benefit of all Sudanese people. Let me also assure you that Austria and the European Union stand ready to support every effort towards a peaceful future for Sudan. It is the aim of the International Community to create a "win-win-situation" for the North and the South.

\section{SC - High-Level Event on Iraq}

Statement by Ambassador Johannes Kyrle, Secretary-General for Foreign Affairs of the Republic of Austria

New York, 15 December 2010

Let me at the outset convey to you, Mr. President, our deep condolences for the passing of Ambassador Richard Holbrooke. We will remember him in particular for his contributions to stability in South-Eastern Europe and, more recently, to peace, security and reconciliation in Afghanistan.

We would like to thank the United States for convening this debate at a crucial moment in Iraq's democratic process. I would also like to convey to you, Mr. Secretary General, Austria's deep appreciation for the outstanding commitment of the United Nations Assistance Mission in Iraq.

Today also marks a milestone in Iraq's return to its rightful status in the international community. As a member of this Council in 1991, Austria voted for a number of the measures we will bring to a close today. Austria supports this step in recognition of the progress achieved by Iraq and in support of reconstruction and economic development for the benefit of its people. Last but not least, we do so as a strong encouragement to the incoming government to fulfill outstanding Chapter VII obligations. 
In 1991, Austria supported resolution 687 and 707 on the premise that actions requested from Iraq represented first steps towards the goal of establishing a zone free from weapons of mass destruction and missiles for their delivery in the Middle East. Today this goal remains as important as ever. In light of Iraq's commitment to non-proliferation and disarmament, we hope that the Comprehensive Test Ban Treaty will be ratified soon and that the welcome provisional application of the Additional Protocol to the IAEA Comprehensive Safeguards Agreement will be followed by its ratification at the earliest possibility. We also expect the destruction of chemical weapons to continue at a determined pace.

As we approach the twentieth anniversary of the liberation of Kuwait, we look to Iraq for a fresh resolve to forge a new partnership with Kuwait that is built on mutual trust.

As border security is paramount to confidence between neighbors, any shadow of doubt regarding the acceptance of the finality and inviolability of common borders should be avoided. We therefore encourage Iraq to re-affirm its commitment to resolution 833 with respect to its land and maritime boundaries.

Efforts should also be reinforced to clarify the fate of missing persons as well as to settle the issue of the restitution of Kuwait's national archives. With Austria's chairmanship of the United Nations Compensation Commission drawing to a close, let me also underline the importance of a functioning mechanism succeeding the Development Fund for Iraq. A continuation of payments into the Compensation Fund needs to be assured in accordance with resolution 1483 until Iraq and Kuwait can come to a consensus on a future compensation regime.

Much has been said today on the fundamental changes we have witnessed in Iraq - a country whose pluralistic traditions and proud history as a cradle of civilizations we admire as much as we ardently support its secure and prosperous future. At the same time, pressing challenges remain which require determined stewardship.

As the European Union Foreign Ministers underlined on 22 November, there is an urgent need for the formation of a stable and representative government which is able to rededicate itself to the pursuit of national reconciliation. Such a government will be vital to responding to the aspirations of all Iraqis - regardless of creed or ethnicity - and to respecting, protecting and promoting their human rights.

We hope to see a renewed resolve by the new federal government and the leadership of the Iraqi Kurdistan Region to come to a compromise on their respective boundaries and competences as well as the distribution of revenues, including from hydrocarbon resources. Austria encourages the continuation of UNAMI's efforts to build trust between Arab and Kurdish communities in the North, in particular by facilitating dialogue on the safeguarding 
of language rights, the rights of detainees and the resolution of property disputes.

Austria condemns all indiscriminate attacks on civilians and reiterates her deep concern about recent attacks on religious minorities including Christian and Muslim places of worship. We urge the Iraqi government to bring the perpetrators to justice and ensure that all Iraqis are adequately protected and able to practice their faith in peace and dignity.

Austria is also concerned about the reported incidents of harassment and intimidation against internally displaced persons in Kirkuk, including threats of forced eviction.

During our term on the Council, we have witnessed a marked decrease in the level of violence, Iraqi Security Forces progressively assuming their responsibilities and the steady anchoring of democratic processes. Those gains have been hard fought for and often challenged by unspeakable violence against innocent civilians. Austria will continue to support the Iraqi people in building a secure, stable, democratic, unified and prosperous country beyond our term on the Council. We look forward to working with a new Iraqi government towards this goal, bilaterally and in the framework of the European Union.

Finally, I wish to assure the Secretary General of Austria's continued support for the important work of Special Representative Ad Melkert and his entire team. Let me also assure you that their safety and security - as for all UN personnel - remains of utmost concern to us.

\section{2. Österreichische Votumserklärungen}

\section{SC - Adoption of Resolution 1871 (2009) / MINURSO}

Explanation of vote by Ambassador Thomas Mayr-Harting, Permanent
Representative of Austria to the United Nations

New York, 30 April 2009

Austria wholeheartedly supports the renewal of the mandate of MINURSO for another 12 months. From our point of view it is very important that the Council has been able to achieve unity in this matter. This provides the SG and his Personal Envoy with the unanimous message of support by the Council that they need for their efforts to bring both parties back to the negotiating table as soon as possible.

In the field of human rights Austria supports a results-oriented approach. Both parties have the obligation to ensure respect for the human rights of the people of Western Sahara. Against this background we believe that the populations in the Territory and in the refugee camps would greatly benefit in their enjoyment of human rights from a new resolve by both parties in the 
negotiation process. A continuous and constructive dialogue with the Office of the High Commissioner for Human Rights, as suggested by the SG, could make it easier to approach the issue of human rights protection and promotion under a confidence-building perspective.

\section{SC - Meeting on DPRK/non-proliferation - Adoption of Resolution 1874 (2009) \\ Explanation of vote by Ambassador Thomas Mayr-Harting, Permanent Representative of Austria to the United Nations}

New York, 12 June 2009

Austria has condemned the DPRK's nuclear test of 25 May 2009 in the strongest terms as a direct violation of Resolution 1718.

The unanimous adoption of the present resolution is a clear, fully appropriate and unequivocal response by this Council and the international community to the DPRK's nuclear test and the threat this poses to international peace and security.

As co-chairs of the Art. XIV-process of the CTBT Costa Rica and Austria welcome the call on the DPRK, as contained in OP 29 of the resolution, to join the "Comprehensive Nuclear-Test-Ban Treaty" (CTBT) at the earliest date.

Costa Rica and Austria believe that the recent events highlight the necessity and urgency of a rapid entry into force of the Comprehensive Nuclear-TestBan Treaty. Furthermore, both countries wish to underline the very valuable contribution made by the Comprehensive Nuclear-Test-Ban Treaty Organisation (CTBTO) in providing reliable data on the DPRK nuclear test of 25 May 2009.

\section{SC - Meeting on Georgia}

Explanation of vote by Ambassador Christian Ebner, Deputy Permanent Representative of Austria to the United Nations

New York, 15 June 2009

Austria regrets that the Council was today not able to agree on the technical roll over resolution before us. We firmly believe that a UN mission monitoring and verifying a security regime continues to be crucial for stability in the region and therefore is in everybody's interest. The mission has played a key role in improving the situation of the internally displaced and refugee population in the area. We therefore attach great importance to a continued UN presence. 
We supported and continue to support the concept of a roll-over resolution so as to give the Council the extra time necessary for negotiations.

We reaffirm our commitment to the sovereignty, independence, and territorial integrity of Georgia within its internationally recognized borders.

The last two technical roll-over resolutions 1839 (2008) and 1866 (2009) which enjoyed consensus on the Council both made explicit reference to resolution 1808 (2008). Austria believes that there have been no major changes in the situation over the last 3 months which would justify the deletion of such a reference in the resolution before us.

\section{SC - Adoption of Resolution 1888 (2009) \\ Statement by Ambassador Thomas Mayr-Harting, Permanent Representa- tive of Austria to the United Nations}

New York, 30 September 2009

Let me first thank you, Madam President, for your personal leadership in the fight against sexual violence in armed conflict. I would also like to thank Ambassador Susan Rice and the US Mission in New York for the energy and determination they have demonstrated in conducting our work on Resolution 1888. We believe that this resolution will significantly contribute to strengthening the United Nations' response to sexual violence in conflict situations.

We see a particular need for the strategic leadership that a newly appointed Special Representative of the Secretary General can provide. Her or his work will help us address this scourge on a systematic basis, in each and every conflict situation where acts of sexual violence occur. With this goal in view we also look forward to the upcoming proposals on enhancing monitoring and reporting on sexual violence to be submitted by the Secretary General whose personal commitment in this matter we highly appreciate.

Today's Resolution will hopefully also pave the way for a much more systematic flow of information between UN peacekeeping missions and the Sanctions Committees of the Council on sexual violence in areas of conflict. Where necessary, the Council must ensure the accountability of those responsible for sexual violence in armed conflict, including through the establishment of commissions of inquiry, referrals to the International Criminal Court and the imposition of targeted measures.

At the same time, it is absolutely unacceptable that civilians, in particular women and girls, continue to fall victim to sexual violence, on a daily basis, also in conflict zones where UN Peacekeeping Missions have a key responsibility for their protection. The fight against sexual violence must therefore also be at the core of our overall protection of civilians' agenda. We thus 
pledge to take this work forward when the Council next considers the protection of civilians in armed conflict during Austria's Security Council Presidency in the month of November.

\section{SC - Adoption of Resolution 1904 (2009)}

\section{Explanation of vote by Ambassador Thomas Mayr-Harting, Permanent Representative of Austria to the United Nations}

New York, 17 December 2009

More than four years ago, the World Summit called upon this Council to provide for fair and clear listing and de-listing procedures for individuals and entities on sanctions lists. As chairman of the 1267-Committee during this last year I have witnessed some of the shortcomings of the present system. I am also aware of the growing number of cases filed by listed individuals and entities before national and regional courts in various parts of the world.

I can therefore also appreciate how very substantially SC resolution 1904 improves the procedures under the 1267 sanctions regime in terms of due process. For the first time ever, individuals and entities seeking a delisting will have the chance to present their cases to an independent and impartial Ombudsperson to be appointed by the Secretary General.

In the new resolution, the Council calls on Committee Members make every effort to provide their reasons when objecting to a delisting request. It also directs the Ombudsperson to forward to petitioners all information about a negative decision provided by the Committee, including explanatory comments. Furthermore, it instructs the Committee to conduct a comprehensive review of all pending issues, so as to resolve them, to the extent possible, by the end of 2010. For these and many other reasons, resolution 1904 represents a significant step forward in improving the fairness and transparency of the 1267 sanctions regime, thus enhancing both its effectiveness and legitimacy.

My delegation would like to thank the United States as the main sponsor of this resolution as well as the other co-sponsors and all the members of the Council for their strong engagement in this very important matter. At the same time, special thanks are due to many other UN Member States who have actively supported this process over many years, in particular the group of "Like-minded States" and the "Friends of the Rule of Law". I would also like to pay tribute to our Belgian predecessor in the Chairmanship of the 1267 Committee who paved the way for today's decision.

Much will depend on the practical implementation of this resolution, especially on the appointment of an eminent Ombudsperson with highest quali- 
fications in fields such as law, human rights, counter-terrorism and sanctions. This is work in progress. This is a case where the decision of the Council "to remain actively seized of the matter" is of particular significance. Therefore, my delegation very much hopes that the Council will continue to show an active interest in the state of the overall work of the 1267-Committee. For this, the bi-annual reports by the Committee to the Council can provide a very useful instrument.

\section{SC - Adoption of Resolution 1907 (2009) \\ Explanation of vote by Ambassador Thomas Mayr-Harting, Permanent Representative of Austria to the United Nations}

New York, 23 December 2009

Austria condemns all acts that attempt to undermine the peace process in Somalia. We urge all parties to join the Djibouti peace process and to support the Transitional Federal Government in its efforts to stabilize the situation in this country.

Austria voted in favour of this resolution. In this context it was important for my delegation that the imposition of targeted sanctions according to this resolution is based on a two-step approach. It is our understanding that any decision on specific designations will be made in the framework of the 751 Sanctions Committee, in accordance with the procedural guarantees of resolution 1844 (2008). We hope that the Eritrean Government uses the intervening time to address the concerns of the international community.

Austria believes that beyond the imposition of sanctions it will be important to continue to search for a solution to the underlying political problems. The government of Eritrea for its part should seize every opportunity to engage in constructive dialogue.

\section{SC - Adoption of Resolution 1920 (2010) / MINURSO \\ Explanation of vote by Ambassador Thomas Mayr-Harting, Permanent Representative of Austria to the United Nations}

New York, 30 April 2010

Austria fully supports the renewal of the mandate of MINURSO for another 12 months and underlines her continued support for the efforts by the Secretary General and his Personal Envoy for the resumption of substantive and result-oriented negotiations. 
We are very glad that the Council was able to maintain consensus. We are grateful to all who cooperated towards this outcome. The unanimous message of support of the Council for the UN's efforts is essential to move the parties beyond the current impasse. Building consensus on this text has been a laborious task in view of the double-track process used to prepare this resolution. We strongly encourage that this modus operandi be reconsidered next year.

Austria would have preferred an explicit support for the Secretary General's call on the parties for continuous and constructive dialogue with the OHCHR with a view to ensuring respect for the human rights of the people of Western Sahara in the Territory and in the refugee camps.

At the same time, Austria strongly supports the resolution's call for progress in the human dimension of the conflict. The programme of visits by air should be continued without further delay and the visits by land inaugurated as called for in the resolution.

We urge the parties to resume their dialogue as soon as possible and to work, in good faith and without preconditions, to overcome the impasse and provide the people of Western Sahara with the perspective of a dignified future of their choice.

Austria will continue to actively support all UN efforts towards a just, lasting and mutually acceptable solution, which will provide for self-determination in accordance with international law. Austria stands ready to assist the SG and the parties in advancing their dialogue towards this goal.

\section{SC - Adoption of Resolution 1923 (2010) / MINURCAT}

\section{Explanation of vote by Ambassador Thomas Mayr-Harting, Permanent Representative of Austria to the United Nations}

New York, 25 May 2010

Austria has voted in favour of today's resolution although we would have preferred a more gradual approach in the drawdown of MINURCAT as well as the continuation of a protection of civilians' mandate for the mission.

At the same time, we note and underline that MINURCAT has at least been authorized to respond to imminent threats of violence to civilians in its immediate vicinity.

We respect the decision of the Government of Chad to assume full responsibility for the security and the protection of the civilian population in Eastern Chad, including refugees, internally displaced persons, returnees and host communities. We appreciate the efforts undertaken by the Chadian authorities to provide the Security Council with a better understanding of their plans and capabilities in this field. We trust that the Government of Chad 
will do its utmost to fulfil its responsibilities and use all available capabilities for the protection of the population in Eastern Chad.

It is of the greatest importance that the joint Government of Chad/ United Nations High Level Working Group plays the key role provided for in this resolution and regularly assesses the situation on the ground with respect to the protection of civilians and humanitarian access.

We call upon the Government of Chad and the UN Secretariat to cooperate as closely as possible within this working group. There is a continued need for the Council to follow developments closely and to be regularly informed about the situation on the ground. Austria believes that the possibility of a further international engagement in Eastern Chad should not be excluded if deemed necessary for humanitarian reasons.

\section{SC - Adoption of Resolution 1929 (2010) / Iran}

\section{Explanation of vote by Ambassador Thomas Mayr-Harting, Permanent Representative of Austria to the United Nations}

New York, 9 June 2010

\section{Mr. President,}

Austria voted in favour of Security Council resolution 1929. A decision of this kind is never one to be taken lightly. From the time that Iran's undeclared nuclear materials and activities were first confirmed by the IAEA in June 2003, Austria had hoped that the matter could be resolved through negotiation. Insufficient cooperation by Iran led to the transmission of the issue to the Security Council in March 2006, and since that time, the Council has adopted a presidential statement and five resolutions. Regrettably, Iran has failed to address the core concerns of the international community and to build confidence in the exclusively peaceful nature of its nuclear programme. Indeed, since the adoption of the last Council resolution in September 2008, the existence of a new undeclared enrichment facility has come to light, and Iran has begun to enrich LEU to 20 percent, to mention but two of the most recent developments. This is all the more unfortunate, as last month the NPT Review Conference here in New York demonstrated a new constructive atmosphere on nuclear disarmament and non-proliferation issues. In the Action Plans adopted on that occasion, all NPT Member States underscored inter alia the importance of cooperating with the International Atomic Energy Agency (IAEA) in questions of compliance.

As I indicated yesterday, Austria - in line with long-standing European Union policy - remains committed to the dual-track approach. In this context, we reiterate our call on Iran to take up the offer for talks with the High Representative of the European Union for Foreign Affairs and Security Policy 
in line with Operative Paragraph 33 of the resolution just adopted. While we believe that the additional measures adopted today are necessary, we continue to stand behind the two major incentives packages put forward in June 2006 and June 2008, and hope that Iran will take up the offer by China, France, Germany, the Russian Federation, the United Kingdom and the United States, as well as the High Representative of the European Union, to resume dialogue on the nuclear issue without preconditions, with a view to seeking a comprehensive solution of this issue. In this context, I wish to highlight in particular the commitment contained in Operative Paragraph 37 to suspend the implementation of measures if and for so long as Iran suspends all enrichment-related and reprocessing activities, as verified by the IAEA, to allow for negotiations in good faith in order to reach an early and mutually acceptable outcome.

Thank you.

\subsection{Ausgewählte Resolutionen und Vorsitzerklärungen}

Folgende Resolutionen und Vorsitzerklärungen sind unter diesem Punkt beigefügt:

1. SR-Resolution 1894 (2009)

2. S/PRST/2010/25

3. SR-Resolution 1888 (2009)

4. S/PRST/2010/22

5. SR-Resolution 1960 (2010)

6. SR-Resolution 1882 (2009)

7. S/PRST/2010/10

8. SR-Resolution 1904 (2009)

9. SR-Resolution 1966 (2010) 


\section{Resolution 1894 (2009)}

\section{Adopted by the Security Council at its 6216th meeting, on 11 November 2009}

\section{The Security Council,}

Reaffirming its commitment to the continuing and full implementation, in a mutually-reinforcing manner, of resolutions 1265 (1999), 1296 (2000), 1325 (2000), 1612 (2005), 1674 (2006), 1738 (2006), 1820 (2008), 1882 (2009), 1888 (2009) and 1889 (2009), and all relevant statements of its President,

Reaffirming its commitment to the Purposes of the Charter of the United Nations as set out in Article 1 (1-4) of the Charter, and to the Principles of the Charter as set out in Article 2 (1-7) of the Charter, including its commitment to the principles of the political independence, sovereign equality and territorial integrity of all States, and respect for the sovereignty of all States,

Noting that this year marks the tenth anniversary of the progressive consideration by the Security Council of the protection of civilians in armed conflict as a thematic issue; and acknowledging the enduring need for the Security Council and Member States to strengthen further the protection of civilians in armed conflict,

Noting further that this year also marks the 60th anniversary of the Geneva Conventions of 1949, which together with their Additional Protocols constitute the basis for the legal framework for the protection of civilians in armed conflict,

Recognizing that States bear the primary responsibility to respect and ensure the human rights of their citizens, as well as all individuals within their territory as provided for by relevant international law,

Reaffirming that parties to armed conflict bear the primary responsibility to take all feasible steps to ensure the protection of civilians,

Reaffirming the relevant provisions of the 2005 World Summit Outcome Document regarding the protection of civilians in armed conflict, including paragraphs 138 and 139 thereof regarding the responsibility to protect populations from genocide, war crimes, ethnic cleansing and crimes against humanity,

Reiterating its deep regret that civilians continue to account for the vast majority of casualties in situations of armed conflict,

* Reissued for technical reasons. 
Stressing the particular impact that armed conflict has on women and children, including as refugees and internally displaced persons, as well as on other civilians who may have specific vulnerabilities including persons with disabilities and older persons, and stressing the protection and assistance needs of all affected civilian populations,

Noting the adoption of the African Union Convention for the Protection and Assistance of Internally Displaced Persons in Africa (2009),

Noting with grave concern the severity and prevalence of constraints on humanitarian access, as well as the frequency and gravity of attacks against humanitarian personnel and objects and the significant implications of such attacks for humanitarian operations,

Recognizing the need for States in or emerging from armed conflict to restore or build accountable security institutions and independent national judicial systems,

Recalling the inclusion of war crimes, crimes against humanity and genocide in the statutes of the ad hoc international criminal tribunals and the Rome Statute of the International Criminal Court, and emphasizing in this regard the principle of complementarity,

Recognizing the importance of reparations programmes in response to serious violations of international humanitarian law and gross human rights violations,

Recognizing the importance of empowering vulnerable civilians through education and training as a means to support efforts to halt and prevent abuses committed against civilians in situations of armed conflict,

Recognizing the valuable contribution to the protection of children in armed conflict by the SRSG on Children and Armed Conflict and the Security Council Working Group on Children and Armed Conflict, including its conclusions and recommendations issued in line with resolution 1612 (2005), and recalling resolution 1882 (2009), which aims to strengthen the protection of children in situations of armed conflict,

Recalling its decision in resolution 1888 (2009) to address violence against women and children in situations of armed conflict by requesting the SecretaryGeneral to appoint a special representative and to identify and take the appropriate measures to deploy rapidly a team of experts to situations of particular concern with respect to sexual violence in armed conflict,

Noting the practice of briefings to Security Council members by the Office for the Coordination of Humanitarian Affairs on behalf of the United Nations' humanitarian community, both through formal and informal channels,

Taking note of the report of the Secretary-General on the protection of civilians of 29 May 2009 (S/2009/277) and its annex on constraints on humanitarian access, which identify the core challenges to the effective protection of civilians, namely enhancing compliance with international law; enhancing compliance by non-State armed groups with their obligations under international law; enhancing protection through more effective and better resourced United Nations peacekeeping and other relevant missions; enhancing humanitarian access; and enhancing accountability for violations, 
Welcoming the proposals, conclusions and recommendations on the protection of civilians included in the report of the Special Committee on Peacekeeping Operations and its Working Group (A/63/19) and the important work conducted by the Security Council Working Group on Peacekeeping Operations, including its efforts aimed at enhancing the implementation of protection mandates,

Recalling the statement of its President of 5 August 2009 (S/PRST/2009/24) and welcoming ongoing efforts to strengthen UN peacekeeping,

Noting that United Nations peacekeeping missions constitute one of several means at the United Nations' disposal to protect civilians in situations of armed conflict,

1. Demands that parties to armed conflict comply strictly with the obligations applicable to them under international humanitarian, human rights and refugee law, as well as to implement all relevant decisions of the Security Council and in this regard, urges them to take all required measures to respect and protect the civilian population and meet its basic needs;

2. Reiterates its condemnation in the strongest terms of attacks in situations of armed conflict directed against civilians as such and other protected persons or objects as well as indiscriminate or disproportionate attacks and the utilisation of the presence of civilians to render certain points, areas or military forces immune from military operations, as flagrant violations of international humanitarian law and demands that all parties immediately put an end to such practices;

3. Notes that the deliberate targeting of civilians as such and other protected persons, and the commission of systematic, flagrant and widespread violations of applicable international humanitarian and human rights law in situations of armed conflict may constitute a threat to international peace and security, and reaffirms in this regard its readiness to consider such situations and, where necessary, to adopt appropriate steps;

4. Reiterates its willingness to respond to situations of armed conflict where civilians are being targeted or humanitarian assistance to civilians is being deliberately obstructed, including through the consideration of appropriate measures at the Security Council's disposal in accordance with the Charter of the United Nations;

5. Reiterates its call on States that have not already done so to consider signing, ratifying or acceding to the relevant instruments of international humanitarian, human rights and refugee law, and to take appropriate legislative, judicial and administrative measures to implement their obligations under these instruments;

6. Demands that all States and parties to armed conflict fully implement all relevant decisions of the Security Council and in this regard cooperate fully with United Nations peacekeeping missions and Country Teams in the follow-up and implementation of these resolutions;

7. Calls upon all parties concerned,

(a) to ensure the widest possible dissemination of information about international humanitarian, human rights and refugee law; 
(b) to provide training for public officials, members of armed forces and armed groups, personnel associated to armed forces, civilian police and law enforcement personnel, and members of the judicial and legal professions; and to raise awareness among civil society and the civilian population on relevant international humanitarian, human rights and refugee law, as well as on the protection, special needs and human rights of women and children in conflict situations, to achieve full and effective compliance;

(c) to ensure that orders and instructions issued to armed forces and other relevant actors are in compliance with applicable international law, and that they are observed, inter alia, by establishing effective disciplinary procedures, central to which must be the strict adherence to the principle of command responsibility to support compliance with international humanitarian law;

(d) to seek, where appropriate, support from United Nations peacekeeping and other relevant missions, as well as United Nations Country Teams and the International Committee of the Red Cross and, where appropriate, other members of the International Red Cross and Red Crescent Movement, on training and awareness raising on international humanitarian, human rights and refugee law;

8. Emphasizes the importance of addressing in its country specific deliberations the compliance of parties to armed conflict with international humanitarian, human rights and refugee law; notes the range of existing methods used, on a case by case basis, for gathering information on alleged violations of applicable international law relating to the protection of civilians and underlines the importance in this regard of receiving information that is timely, objective, accurate and reliable;

9. Considers the possibility, to this end, of using the International FactFinding Commission established by Article 90 of the First Additional Protocol to the Geneva Conventions;

10. Affirms its strong opposition to impunity for serious violations of international humanitarian law and human rights law and emphasizes in this context the responsibility of States to comply with their relevant obligations to end impunity and to thoroughly investigate and prosecute persons responsible for war crimes, genocide, crimes against humanity or other serious violations of international humanitarian law in order to prevent violations, avoid their recurrence and seek sustainable peace, justice, truth and reconciliation;

11. Recalls that accountability for such serious crimes must be ensured by taking measures at the national level, and by enhancing international cooperation in support of national mechanisms, draws attention to the full range of justice and reconciliation mechanisms to be considered, including national, international and "mixed" criminal courts and tribunals, and truth and reconciliation commissions, as well as national reparation programs for victims and institutional reforms; and underlines the Security Council's role in ending impunity;

12. Reaffirms the Security Council's role in promoting an environment that is conducive to the facilitation of humanitarian access to those in need;

13. Stresses the importance for all, within the framework of humanitarian assistance, of upholding and respecting the humanitarian principles of humanity, neutrality, impartiality and independence; 
14. Stresses the importance for all parties to armed conflict to cooperate with humanitarian personnel in order to allow and facilitate access to civilian populations affected by armed conflict;

15. Expresses its intention to:

(a) Call on parties to armed conflict to comply with the obligations applicable to them under international humanitarian law to take all required steps to protect civilians and to facilitate the rapid and unimpeded passage of relief consignments, equipment and personnel,

(b) Mandate UN peacekeeping and other relevant missions, where appropriate, to assist in creating conditions conducive to safe, timely and unimpeded humanitarian assistance,

16. Further expresses its intention to:

(a) Consistently condemn and call for the immediate cessation of all acts of violence and other forms of intimidation deliberately directed against humanitarian personnel,

(b) Call on parties to armed conflict to comply with the obligations applicable to them under international humanitarian law to respect and protect humanitarian personnel and consignments used for humanitarian relief operations,

(c) Take appropriate steps in response to deliberate attacks against humanitarian personnel;

17. Invites the Secretary-General to continue the systematic monitoring and analysis of constraints on humanitarian access, to include as appropriate observations and recommendations in his briefings and country-specific reports to the Council;

18. Recalls its determination to upgrade the strategic oversight of peacekeeping operations mindful of the important role peacekeeping operations play for the protection of civilians and reaffirms its support to the efforts made by the Secretary-General to review peacekeeping operations and to provide enhanced planning and support and renews its encouragement to deepen these efforts, in partnership with troop and police contributing countries and other relevant stakeholders;

19. Reaffirms its practice of ensuring that mandates of UN peacekeeping and other relevant missions include, where appropriate and on a case-by-case basis, provisions regarding the protection of civilians, stresses that mandated protection activities must be given priority in decisions about the use of available capacity and resources, including information and intelligence resources, in the implementation of mandates; and recognizes, that the protection of civilians when and as mandated requires a coordinated response from all relevant mission components;

20. Reaffirms also the importance of entrusting peacekeeping and other relevant missions that are tasked with the protection of civilians with clear, credible and achievable mandates, based on accurate and reliable information on the situation on the ground, and a realistic assessment of threats against civilians and missions, made in consultation with all relevant stakeholders, and further reaffirms the importance of a greater awareness in the Security Council of the resource and 
field support implications of its decisions and stresses the necessity to ensure the execution of the afore-mentioned mandates to protect civilians in the field;

21. Recognizes the necessity to take into account the protection needs of civilians in situations of armed conflict, in particular women and children, in the early phase of mandate drafting and throughout the lifecycle of United Nations peacekeeping and other relevant missions and in this regard underlines the importance of engagement with the countries concerned and of close consultation with the Secretariat, troop and police contributing countries, and other relevant actors;

22. Recognizes also the need for comprehensive operational guidance on peacekeeping missions' tasks and responsibilities in the implementation of protection of civilians mandates and requests the Secretary-General to develop in close consultation with Member States including troop and police contributing countries and other relevant actors, an operational concept for the protection of civilians, and to report back on progress made;

23. Requests the Secretary-General, in consultation with relevant actors, to ensure that peacekeeping missions with protection of civilians' mandates, in keeping with the strategic plans that guide their deployment, conduct mission-wide planning, pre-deployment training, and senior leadership training on the protection of civilians and requests troop and police contributing countries to ensure the provision of appropriate training of their personnel participating in UN peacekeeping and other relevant missions to heighten the awareness and responsiveness to protection concerns, including training on HIV/AIDS and zero tolerance of sexual exploitation and abuse in UN peacekeeping missions;

24. Requests the Secretary-General to ensure that all relevant peacekeeping missions with protection mandates incorporate comprehensive protection strategies into the overall mission implementation plans and contingency plans which include assessments of potential threats and options for crisis response and risk mitigation and establish priorities, actions and clear roles and responsibilities under the leadership and coordination of the SRSG, with the full involvement of all relevant actors and in consultation with United Nations Country teams;

25. Requests the Secretary-General to ensure that United Nations missions provide local communities with adequate information with regard to the role of the mission and in this regard ensure coordination between a United Nations mission and relevant humanitarian agencies;

26. Takes note of practical measures taken by ongoing peacekeeping missions and United Nations Country Teams to enhance the protection of civilians in the field, and requests the Secretary-General to include best practice in his next report on protection of civilians to the Council;

27. Reaffirms its practice of requiring benchmarks, as and where appropriate, to measure and review progress made in the implementation of peacekeeping mandates and stresses the importance of including indicators of progress regarding the protection of civilians in such benchmarks for relevant missions;

28. Emphasizes the need for a comprehensive approach to facilitate the implementation of protection mandates through promoting economic growth, good governance, democracy, the rule of law, and respect for, and protection of human 
rights, and in this regard, urges the cooperation of Member States and underlines the importance of a coherent, comprehensive and coordinated approach by the principal organs of the United Nations, cooperating with one another and within their respective mandates;

29. Notes that the excessive accumulation and destabilizing effect of small arms and light weapons pose a considerable impediment to the provision of humanitarian assistance and have a potential to exacerbate and prolong conflicts, endanger civilians and undermine security and the confidence required for a return to peace and stability, calls on parties to armed conflict to take all feasible precautions to protect the civilian population, including children, from the effects of landmines and other explosive remnants of war, and in this regard, encourages the international community to support country efforts in clearing landmines and other explosive remnants of war and to provide assistance for the care, rehabilitation and economic and social reintegration of victims, including persons with disabilities;

30. Reiterates the importance of the Aide Memoire on the protection of civilians (S/PRST/2009/1) as a practical tool that provides a basis for improved analysis and diagnosis of key protection issues, particularly during deliberations on peacekeeping mandates and stresses the need to implement the approaches set out therein on a more regular and consistent basis, taking into account the particular circumstances of each conflict situation;

31. Recognizes the important role of the Secretary-General in providing timely information to the Security Council on protection of civilians in armed conflict in particular through thematic and country specific reports and through briefings;

32. Requests the Secretary-General to include in his reports to the Council on country-specific situations more comprehensive and detailed information relating to the protection of civilians in armed conflict, including on protection-related incidents and actions taken by parties to armed conflict to implement their obligations to respect and protect the civilian population, including information specific to the protection needs of refugees, internally displaced persons, women, children and other vulnerable groups;

33. Requests the Secretary-General to develop guidance for UN peacekeeping and other relevant missions on the reporting on the protection of civilians in armed conflict with a view to streamlining reporting and enhancing the Council's monitoring and oversight of the implementation of protection mandates of UN peacekeeping and other missions;

34. Stresses the importance of consultation and cooperation between the United Nations, the International Committee of the Red Cross and other relevant organisations including regional organisations to improve the protection of civilians in armed conflict;

35. Requests the Secretary-General to submit his next report on the protection of civilians in armed conflict by November 2010;

36. Decides to remain seized of the matter. 


\section{Statement by the President of the Security Council}

At the 6427th meeting of the Security Council, held on 22 November 2010, in connection with the Council's consideration of the item entitled "Protection of civilians in armed conflict", the President of the Security Council made the following statement on behalf of the Council:

"The Security Council reaffirms its commitment regarding the protection of civilians in armed conflict, to the continuing and full implementation, in a mutually-reinforcing manner of all previous relevant resolutions and statements of its president including resolutions 1265 (1999), 1296 (2000), 1325 (2000), 1612 (2005), 1674(2006), 1738 (2006), 1820 (2008), 1882 (2009) 1888 (2009), 1889 (2009) 1894 (2009), noting in particular that resolution 1894 marked a significant step in providing guidance to ensure the effective protection of civilians on the ground.

"The Security Council takes note with appreciation of the report of the Secretary-General on the protection of civilians of 11 November 2010 (S/2010/579) and the recommendations contained therein.

"The Security Council recalls that on 15 March 2002 an Aide Memoire was adopted, as a practical tool that provides a basis for improved analysis and diagnosis of key protection issues. The Security Council adopts the updated aide memoire contained in the annex to this Statement of its President and stresses the need to continue its use on a more systematic and consistent basis.

"The Security Council recognizes that States bear the primary responsibility to respect and ensure the human rights of all individuals within their territory and subject to their jurisdiction as provided for by relevant international law.

"The Security Council reaffirms that parties to armed conflict bear the primary responsibility to take all feasible steps to ensure the protection of affected civilians and urges parties to armed conflict to meet their basic needs, and give attention to the specific needs of women and children, refugees, internally displaced persons, as well as other civilians who may have specific vulnerabilities including persons with disabilities and older persons.

"The Security Council emphasizes that the promotion of peace processes and the achievement of sustainable peace and development as well as respect for human rights and the rule of law are of utmost importance for the long term protection of civilians. 


\section{Anhang}

"The Security Council remains committed to addressing the impact of armed conflict on civilians, in particular women and children. The Council expresses its deep regret that civilians continue to account for the vast majority of casualties in situations of armed conflict, including as a result of deliberate targeting, indiscriminate or disproportionate attacks and sexual and gender based violence, as well as other acts that violate applicable international law. The Council demands that all relevant parties immediately put an end to such practices and reaffirms its readiness to adopt appropriate measures.

"The Security Council notes with concern the humanitarian impact of conflict, in or near densely populated areas, and calls on parties to armed conflict to give protection to the civilian population in accordance with applicable international humanitarian law.

"The Security Council reiterates its strong condemnation of all violations of applicable international law and demands that parties to armed conflict comply strictly with the obligations applicable to them under international humanitarian, human rights and refugee law, as well as to implement all relevant decisions of the Security Council. The Security Council emphasizes in this context the responsibility of States to comply with their relevant obligations to end impunity and notes that the fight against impunity for the most serious crimes of international concern has been strengthened through prosecution of these crimes in national, international and "mixed" criminal courts and tribunals, commissions of inquiry as well as specialized chambers in national tribunals. The Council takes note of the stocktaking of international criminal justice undertaken by the first Review Conference of the Rome Statute held in Kampala, Uganda from 31 May to 11 June 2010. The Security Council also draws attention to the full range of justice and reconciliation mechanisms, including truth and reconciliation commissions, national reparation programmes and institutional reforms.

"The Security Council recognises the needs of civilians under foreign occupation and stresses further, in this regard, the responsibilities of the occupying power in full compliance with international humanitarian law.

"The Council reiterates the importance for all, within the framework of humanitarian assistance, of upholding and respecting the humanitarian principles of humanity, neutrality, impartiality and independence. The Security Council condemns and calls for the cessation of all acts of violence and other forms of intimidation deliberately directed at humanitarian personnel as such and calls on all parties to conflict to comply with the obligations applicable to them under international humanitarian law to respect and protect humanitarian personnel and relief consignments. The Council underlines in this respect the importance to continue the systematic monitoring and analysis of constraints on humanitarian access.

"The Security Council stresses the importance of achieving durable solutions for refugees and internally displaced persons, in particular their voluntary, safe and dignified return, or local integration or resettlement.

"The Security Council welcomes the proposals, conclusions and recommendations on the protection of civilians included in the report of the Special Committee on Peacekeeping Operations (A/64/19). The Council 
stresses the importance of ensuring engagement by senior mission leadership on the protection of civilians, with a view to ensuring that all mission components and all levels of the chain of command are properly informed of and involved in the mission's protection mandate and their relevant responsibilities. The Council welcomes progress made by the SecretaryGeneral in elaborating a conceptual framework, outlining resource and capability requirements and developing operational tools for the implementation of protection of civilians mandates. The Council emphasises the importance of improving pre-deployment training for peacekeeping personnel on the protection of civilians. The Council encourages troop and police contributing countries to make full use of and provide feedback on these important materials.

"The Security Council underlines that, in order to carry out their mandate, missions must communicate effectively with local communities and have the capacity to do so. The Council underlines, in this context, the importance of taking into account gender sensitivities and of making full use of all the tools available to the mission, in particular its Public Information and Civil Affairs components, such as civil affairs officers, community liaison interpreters, and radio.

"The Security Council reaffirms its practice of requiring benchmarks to measure and review progress made in the implementation of peacekeeping mandates, underlines the importance of clear benchmarks in the context of drawdown in peacekeeping missions, and stresses the importance of including indicators of progress regarding the protection of civilians in such benchmarks for relevant missions.

"The Security Council recognises the need for systematic monitoring and reporting on progress to protect civilians in armed conflict. The Security Council further reiterates its request to the Secretary-General to develop guidance for peacekeeping and other relevant missions on the reporting of the protection of civilians in armed conflict. The Security Council reiterates its request to the Secretary-General to include in his reports on country-specific situations more comprehensive and detailed information relating to protection of civilians in armed conflict.

"The Security Council notes the practice of briefings to Security Council members by the Office for the Coordination of Humanitarian Affairs on behalf of the United Nations humanitarian community.

"The Security Council takes note of the Montreux Document on pertinent international legal obligations and good practices for States related to operations of private military and security companies during armed conflict.

"The Security Council emphasizes that all civilians affected by armed conflict, including those suffering losses as a result of lawful acts under international law, deserve assistance and recognition in respect of their inherent dignity as human beings.

"The Security Council requests the Secretary-General to submit his next report on the protection of civilians by May 2012." 


\section{Anhang}

\section{Security Council}

Distr.: General

30 September 2009

Resolution 1888 (2009)

\section{Adopted by the Security Council at its 6195th meeting, on 30 September 2009}

\section{The Security Council,}

Reaffirming its commitment to the continuing and full implementation of resolutions 1325 (2000), 1612 (2005), 1674 (2006), 1820 (2008) and 1882 (2009) and all relevant statements of its President,

Welcoming the report of the Secretary-General of 16 July 2009 (S/2009/362), but remaining deeply concerned over the lack of progress on the issue of sexual violence in situations of armed conflict in particular against women and children, notably against girls, and noting as documented in the Secretary-General's report that sexual violence occurs in armed conflicts throughout the world,

Reiterating deep concern that, despite its repeated condemnation of violence against women and children including all forms of sexual violence in situations of armed conflict, and despite its calls addressed to all parties to armed conflict for the cessation of such acts with immediate effect, such acts continue to occur, and in some situations have become systematic or widespread,

Recalling the commitments of the Beijing Declaration and Platform for Action $(\mathrm{A} / 52 / 231)$ as well as those contained in the outcome document of the twenty-third Special Session of the United Nations General Assembly entitled "Women 2000: Gender Equality, Development and Peace for the Twenty-First Century" (A/S-23/10/Rev.1), in particular those concerning women and armed conflict,

Reaffirming the obligations of States Parties to the Convention on the Elimination of All Forms of Discrimination against Women, the Optional Protocol thereto, the Convention on the Rights of the Child and the Optional Protocols thereto, and urging states that have not yet done so to consider ratifying or acceding to them,

Recalling that international humanitarian law affords general protection to women and children as part of the civilian population during armed conflicts and special protection due to the fact that they can be placed particularly at risk,

\footnotetext{
* Reissued for technical reasons on 22 June 2010.
} 
Recalling the responsibilities of States to end impunity and to prosecute those responsible for genocide, crimes against humanity, war crimes and other egregious crimes perpetrated against civilians, and in this regard, noting with concern that only limited numbers of perpetrators of sexual violence have been brought to justice, while recognizing that in conflict and in post conflict situations national justice systems may be significantly weakened,

Reaffirming that ending impunity is essential if a society in conflict or recovering from conflict is to come to terms with past abuses committed against civilians affected by armed conflict and to prevent future such abuses, drawing attention to the full range of justice and reconciliation mechanisms to be considered, including national, international and "mixed" criminal courts and tribunals and truth and reconciliation commissions, and noting that such mechanisms can promote not only individual responsibility for serious crimes, but also peace, truth, reconciliation and the rights of the victims,

Recalling the inclusion of a range of sexual violence offences in the Rome Statute of the International Criminal Court and the statutes of the ad hoc international criminal tribunals,

Stressing the necessity for all States and non-State parties to conflicts to comply fully with their obligations under applicable international law, including the prohibition on all forms of sexual violence,

Recognizing the need for civilian and military leaders, consistent with the principle of command responsibility, to demonstrate commitment and political will to prevent sexual violence and to combat impunity and enforce accountability, and that inaction can send a message that the incidence of sexual violence in conflicts is tolerated,

Emphasizing the importance of addressing sexual violence issues from the outset of peace processes and mediation efforts, in order to protect populations at risk and promote full stability, in particular in the areas of pre-ceasefire humanitarian access and human rights agreements, ceasefires and ceasefire monitoring, Disarmament, Demobilization and Reintegration (DDR), Security Sector Reform (SSR) arrangements, justice and reparations, post-conflict recovery and development,

Noting with concern the underrepresentation of women in formal peace processes, the lack of mediators and ceasefire monitors with proper training in dealing with sexual violence, and the lack of women as Chief or Lead peace mediators in United Nations-sponsored peace talks,

Recognizing that the promotion and empowerment of women and that support for women's organizations and networks are essential in the consolidation of peace to promote the equal and full participation of women and encouraging Member States, donors, and civil society, including non-governmental organizations, to provide support in this respect,

Welcoming the inclusion of women in peacekeeping missions in civil, military and police functions, and recognizing that women and children affected by armed conflict may feel more secure working with and reporting abuse to women in peacekeeping missions, and that the presence of women peacekeepers may encourage local women to participate in the national armed and security forces, 


\section{Anhang}

thereby helping to build a security sector that is accessible and responsive to all, especially women,

Welcoming the efforts of the Department of Peacekeeping Operations to develop gender guidelines for military personnel in peacekeeping operations to facilitate the implementation of resolutions 1325 (2000) and 1820 (2008), and operational guidance to assist civilian, military and police components of peacekeeping missions to effectively implement resolution 1820 (2008),

Having considered the report of the Secretary-General of 16 July 2009 (S/2009/362) and stressing that the present resolution does not seek to make any legal determination as to whether situations that are referred to in the SecretaryGeneral's report are or are not armed conflicts within the context of the Geneva Conventions and the Additional Protocols thereto, nor does it prejudge the legal status of the non-State parties involved in these situations,

Recalling the Council's decision in resolution 1882 of 4 August 2009 (S/RES/1882) to expand the Annexed list in the Secretary General's annual report on Children and Armed Conflict of parties in situations of armed conflict engaged in the recruitment or use of children in violation of international law to also include those parties to armed conflict that engage, in contravention of applicable international law, in patterns of killing and maiming of children and/or rape and other sexual violence against children, in situations of armed conflict,

Noting the role currently assigned to the Office of the Special Adviser on Gender Issues to monitor implementation of resolution 1325 and to promote gender mainstreaming within the United Nations system, women's empowerment and gender equality, and expressing the importance of effective coordination within the United Nations system in these areas,

Recognizing that States bear the primary responsibility to respect and ensure the human rights of their citizens, as well as all individuals within their territory as provided for by relevant international law,

Reaffirming that parties to armed conflict bear the primary responsibility to take all feasible steps to ensure the protection of affected civilians,

Reiterating its primary responsibility for the maintenance of international peace and security and, in this connection, its commitment to continue to address the widespread impact of armed conflict on civilians, including with regard to sexual violence,

1. Reaffirms that sexual violence, when used or commissioned as a tactic of war in order to deliberately target civilians or as a part of a widespread or systematic attack against civilian populations, can significantly exacerbate situations of armed conflict and may impede the restoration of international peace and security; affirms in this regard that effective steps to prevent and respond to such acts of sexual violence can significantly contribute to the maintenance of international peace and security; and expresses its readiness, when considering situations on the agenda of the Council, to take, where necessary, appropriate steps to address widespread or systematic sexual violence in situations of armed conflict;

2. Reiterates its demand for the complete cessation by all parties to armed conflict of all acts of sexual violence with immediate effect; 
3. Demands that all parties to armed conflict immediately take appropriate measures to protect civilians, including women and children, from all forms of sexual violence, including measures such as, inter alia, enforcing appropriate military disciplinary measures and upholding the principle of command responsibility, training troops on the categorical prohibition of all forms of sexual violence against civilians, debunking myths that fuel sexual violence and vetting candidates for national armies and security forces to ensure the exclusion of those associated with serious violations of international humanitarian and human rights law, including sexual violence;

4. Requests that the United Nations Secretary-General appoint a Special Representative to provide coherent and strategic leadership, to work effectively to strengthen existing United Nations coordination mechanisms, and to engage in advocacy efforts, inter alia with governments, including military and judicial representatives, as well as with all parties to armed conflict and civil society, in order to address, at both headquarters and country level, sexual violence in armed conflict, while promoting cooperation and coordination of efforts among all relevant stakeholders, primarily through the inter-agency initiative "United Nations Action Against Sexual Violence in Conflict";

5. Encourages the entities comprising UN Action Against Sexual Violence in Conflict, as well as other relevant parts of the United Nations system, to support the work of the aforementioned Special Representative of the Secretary-General and to continue and enhance cooperation and information sharing among all relevant stakeholders in order to reinforce coordination and avoid overlap at the headquarters and country levels and improve system-wide response;

6. Urges States to undertake comprehensive legal and judicial reforms, as appropriate, in conformity with international law, without delay and with a view to bringing perpetrators of sexual violence in conflicts to justice and to ensuring that survivors have access to justice, are treated with dignity throughout the justice process and are protected and receive redress for their suffering;

7. Urges all parties to a conflict to ensure that all reports of sexual violence committed by civilians or by military personnel are thoroughly investigated and the alleged perpetrators brought to justice, and that civilian superiors and military commanders, in accordance with international humanitarian law, use their authority and powers to prevent sexual violence, including by combating impunity;

8. Calls upon the Secretary-General to identify and take the appropriate measures to deploy rapidly a team of experts to situations of particular concern with respect to sexual violence in armed conflict, working through the United Nations presence on the ground and with the consent of the host government, to assist national authorities to strengthen the rule of law, and recommends making use of existing human resources within the United Nations system and voluntary contributions, drawing upon requisite expertise, as appropriate, in the rule of law, civilian and military judicial systems, mediation, criminal investigation, security sector reform, witness protection, fair trial standards, and public outreach; to, inter alia:

(a) Work closely with national legal and judicial officials and other personnel in the relevant governments' civilian and military justice systems to 
address impunity, including by the strengthening of national capacity, and drawing attention to the full range of justice mechanisms to be considered;

(b) Identify gaps in national response and encourage a holistic national approach to address sexual violence in armed conflict, including by enhancing criminal accountability, responsiveness to victims, and judicial capacity;

(c) Make recommendations to coordinate domestic and international efforts and resources to reinforce the government's ability to address sexual violence in armed conflict;

(d) Work with the United Nations Mission, Country Team, and the aforementioned Special Representative of the Secretary-General as appropriate towards the full implementation of the measures called for by resolution 1820 (2008);

9. Encourages States, relevant United Nations entities and civil society, as appropriate, to provide assistance in close cooperation with national authorities to build national capacity in the judicial and law enforcement systems in situations of particular concern with respect to sexual violence in armed conflict;

10. Reiterates its intention, when adopting or renewing targeted sanctions in situations of armed conflict, to consider including, where appropriate, designation criteria pertaining to acts of rape and other forms of sexual violence; and calls upon all peacekeeping and other relevant United Nations missions and United Nations bodies, in particular the Working Group on Children and Armed Conflict, to share with relevant United Nations Security Council sanctions committees, including through relevant United Nations Security Council Sanction Committees' monitoring groups and groups of experts, all pertinent information about sexual violence;

11. Expresses its intention to ensure that resolutions to establish or renew peacekeeping mandates contain provisions, as appropriate, on the prevention of, and response to, sexual violence, with corresponding reporting requirements to the Council;

12. Decides to include specific provisions, as appropriate, for the protection of women and children from rape and other sexual violence in the mandates of United Nations peacekeeping operations, including, on a case-by-case basis, the identification of women's protection advisers (WPAs) among gender advisers and human rights protection units, and requests the Secretary-General to ensure that the need for, and the number and roles of WPAs are systematically assessed during the preparation of each United Nations peacekeeping operation;

13. Encourages States, with the support of the international community, to increase access to health care, psychosocial support, legal assistance and socio economic reintegration services for victims of sexual violence, in particular in rural areas;

14. Expresses its intention to make better usage of periodical field visits to conflict areas, through the organization of interactive meetings with the local women and women's organizations in the field about the concerns and needs of women in areas of armed conflict;

15. Encourages leaders at the national and local level, including traditional leaders where they exist and religious leaders, to play a more active role in 
sensitizing communities on sexual violence to avoid marginalization and stigmatization of victims, to assist with their social reintegration, and to combat a culture of impunity for these crimes;

16. Urges the Secretary General, Member States and the heads of regional organizations to take measures to increase the representation of women in mediation processes and decision-making processes with regard to conflict resolution and peacebuilding;

17. Urges that issues of sexual violence be included in all United Nationssponsored peace negotiation agendas, and also urges inclusion of sexual violence issues from the outset of peace processes in such situations, in particular in the areas of pre-ceasefires, humanitarian access and human rights agreements, ceasefires and ceasefire monitoring, DDR and SSR arrangements, vetting of armed and security forces, justice, reparations, and recovery/development;

18. Reaffirms the role of the Peacebuilding Commission in promoting inclusive gender-based approaches to reducing instability in post-conflict situations, noting the important role of women in rebuilding society, and urges the Peacebuilding Commission to encourage all parties in the countries on its agenda to incorporate and implement measures to reduce sexual violence in post-conflict strategies;

19. Encourages Member States to deploy greater numbers of female military and police personnel to United Nations peacekeeping operations, and to provide all military and police personnel with adequate training to carry out their responsibilities;

20. Requests the Secretary-General to ensure that technical support is provided to troop and police contributing countries, in order to include guidance for military and police personnel on addressing sexual violence in predeployment and induction training;

21. Requests the Secretary-General to continue and strengthen efforts to implement the policy of zero tolerance of sexual exploitation and abuse in United Nations peacekeeping operations; and urges troop and police contributing countries to take appropriate preventative action, including predeployment and in-theater awareness training, and other action to ensure full accountability in cases of such conduct involving their personnel;

22. Requests that the Secretary-General continue to direct all relevant United Nations entities to take specific measures to ensure systematic mainstreaming of gender issues within their respective institutions, including by ensuring allocation of adequate financial and human resources within all relevant offices and departments and on the ground, as well as to strengthen, within their respective mandates, their cooperation and coordination when addressing the issue of sexual violence in armed conflict;

23. Urges relevant Special Representatives and the Emergency Relief Coordinator of the Secretary-General, with strategic and technical support from the UN Action network, to work with Member States to develop joint GovernmentUnited Nations Comprehensive Strategies to Combat Sexual Violence, in consultation with all relevant stakeholders, and to regularly provide updates on this in their standard reporting to Headquarters; 
24. Requests that the Secretary-General ensure more systematic reporting on incidents of trends, emerging patterns of attack, and early warning indicators of the use of sexual violence in armed conflict in all relevant reports to the Council, and encourages the Special Representatives of the Secretary-General, the Emergency Relief Coordinator, the High Commissioner for Human Rights, the Special Rapporteur on Violence against Women, and the Chairperson(s) of UN Action to provide, in coordination with the aforementioned Special Representative, additional briefings and documentation on sexual violence in armed conflict to the Council;

25. Requests the Secretary-General to include, where appropriate, in his regular reports on individual peacekeeping operations, information on steps taken to implement measures to protect civilians, particularly women and children, against sexual violence;

26. Requests the Secretary-General, taking into account the proposals contained in his report as well as any other relevant elements, to devise urgently and preferably within three months, specific proposals on ways to ensure monitoring and reporting in a more effective and efficient way within the existing United Nations system on the protection of women and children from rape and other sexual violence in armed conflict and post-conflict situations, utilizing expertise from the United Nations system and the contributions of national Governments, regional organizations, non-governmental organizations in their advisory capacity and various civil society actors, in order to provide timely, objective, accurate and reliable information on gaps in United Nations entities response, for consideration in taking appropriate action;

27. Requests that the Secretary-General continue to submit annual reports to the Council on the implementation of Resolution 1820 (2008) and to submit his next report by September of 2010 on the implementation of this resolution and Resolution 1820 (2008) to include, inter alia:

(a) a detailed coordination and strategy plan on the timely and ethical collection of information;

(b) updates on efforts by United Nations Mission focal points on sexual violence to work closely with the Resident Coordinator/Humanitarian Coordinator (RC/HC), the United Nations Country Team, and, where appropriate, the aforementioned Special Representative and/or the Team of Experts, to address sexual violence;

(c) information regarding parties to armed conflict that are credibly suspected of committing patterns of rape or other forms of sexual violence, in situations that are on the Council's agenda;

28. Decides to review, taking into account the process established by General Assembly resolution 63/311 regarding a United Nations composite gender entity, the mandates of the Special Representative requested in operative paragraph 4 and the Team of Experts in operative paragraph 8 within two years, and as appropriate thereafter;

29. Decides to remain actively seized of the matter. 


\section{Statement by the President of the Security Council}

At the 6411th meeting of the Security Council, held on 26 October 2010, in connection with the Council's consideration of the item entitled "Women and peace and security", the President of the Security Council made the following statement on behalf of the Council:

"The Security Council, meeting on the tenth Anniversary of the adoption of its resolution 1325 (2000), reaffirms its commitment to the continuing and full implementation, in a mutually reinforcing manner, of resolutions 1325 (2000), 1612 (2005), 1674 (2006), 1820 (2008), 1882 (2009), 1888 (2009), 1889 (2009), 1894 (2009) and all relevant Statements of its Presidents.

"The Security Council welcomes the report of the Secretary-General on Women and Peace and Security (S/2010/498), and the analysis it contains on progress in implementing resolution 1325 (2000).

"The Security Council welcomes General Assembly resolution A/RES/64/289 establishing the UN Entity for Gender Equality and the Empowerment of Women (UN Women) that will be fully operational in January 2011. The Council invites UN Women to regularly contribute to its work on women and peace and security and notes the valuable role it will play in supporting women's roles in peacebuilding and the prevention of sexual violence in conflict, including through coordination and coherence in policy and programming for women and girls. It welcomes the appointment of Ms. Michele Bachelet as its head.

"The Security Council reiterates its strong condemnation of all violations of applicable international law committed against women and girls in situations of armed conflict and post-conflict situations, including rape, other forms of sexual and gender-based violence and killing and maiming that contravene international law. The Council urges the complete cessation by all parties of such acts with immediate effect and also urges Member States to bring to justice those responsible for crimes of this nature. Their efforts to combat impunity must be matched with assistance and redress to victims. In this regard, it reiterates its support for the mandates of the Special Representatives of the Secretary-General on Sexual Violence in Conflict and for Children and Armed Conflict and encourages them to continue to ensure full transparency, cooperation and coordination of their efforts.

"The Security Council notes that the fight against impunity for the most serious crimes of international concern committed against women and girls has been

\footnotetext{
* Reissued for technical reasons.
} 


\section{Anhang}

strengthened through the work of the International Criminal Court, ad hoc and mixed tribunals, as well as specialized chambers in national tribunals and takes note of the stocktaking of international criminal justice undertaken by the first Review Conference of the Rome Statute held in Kampala, Uganda from 31 May to 11 June 2010. The Council intends to enhance its efforts to fight impunity and uphold accountability for serious crimes against women and girls with appropriate means and draws attention to the full range of justice and reconciliation mechanisms to be considered, including national, international and mixed criminal courts and tribunals, truth and reconciliation commissions as well as national reparation programs for victims, institutional reforms and traditional dispute resolution mechanisms.

"The Security Council recognizes the continued challenges and welcomes the many efforts to implement resolution 1325 (2000) detailed in the SecretaryGeneral's report, in particular positive examples of efforts to engage with women's civil society groups in conflict resolution and peacebuilding, and to protect women and girls from sexual and gender-based violence.

"The Security Council notes with grave concern that women and girls are disproportionately affected by conflict, and that women's participation at all stages of peace processes and in the implementation of peace accords remains too low, despite the vital role of women in the prevention and resolution of conflicts and in rebuilding their societies. The Council recognizes the need to facilitate the full and effective participation of women in these areas and stresses that the full and effective participation of women is very important for sustainability of peace processes.

"The Security Council welcomes the efforts of Member States to implement its resolution $1325(2000)$ at the national level, including the increase in the number of States that have formulated or revised national action plans and strategies, and encourages Member States to continue to pursue such implementation.

"The Security Council welcomes the concrete commitments made by a number of Member States at the present Ministerial Open Debate on 26 October 2010 to increase their efforts to implement its resolution 1325 (2000) and invites those Member States and any other Member States that wish to do so to regularly review implementation of this resolution and to report to the Security Council on progress made as appropriate.

"The Security Council supports taking forward, including by relevant UN entities, the set of indicators contained in the report of the Secretary-General $(\mathrm{S} / 2010 / 498)$ for use as an initial framework to track implementation of its resolution 1325 in situations of armed conflict and post-conflict and other situations relevant to the implementation of resolution 1325, as appropriate, and taking into account the specificity of each country.

"The Security Council recognizes the need for consistent implementation of resolution 1325 (2000) in its own work and for monitoring progress in implementation. In this regard the Security Council underlines the need for timely and systematic reporting on women and peace and security issues and urges the Secretary-General to ensure that country-specific and relevant thematic issues reports and briefings, provide information on women and peace and security issues 
and on the implementation of resolution 1325 (2000) using this set of indicators, as appropriate.

"The Security Council encourages Member States to take into account the set of indicators contained in the Annex of the Report of the Secretary-General on Women and Peace and Security (S/2010/498), as appropriate, in implementing Security Council resolution 1325 (2000) and subsequent resolutions on women and peace and security.

"The Security Council reiterates its demand to all parties to armed conflict to immediately and completely cease all forms of violence against women and girls, including acts of sexual violence.

"The Security Council encourages Member States to deploy greater numbers of female military and police personnel to United Nations peacekeeping operations, and to provide all military and police personnel with adequate training to carry out their responsibilities. The Council requests the Secretary-General to continue and strengthen efforts to implement the policy of zero tolerance on sexual exploitation and abuse by United Nations peacekeeping and humanitarian personnel. The Council requests the Secretary-General to continue to provide and deploy guidance on addressing sexual violence for predeployment and inductive training of military and police personnel, and to assist missions in developing situation-specific procedures to address sexual violence at the field level and to ensure that technical support is provided to troop and police contributing countries in order to include guidance for military and police personnel on addressing sexual violence in predeployment and induction training. The Security Council welcomes the work of gender and women protection advisers appointed to peacekeeping missions. The Council looks forward to considering the annual report of the Secretary-General on the implementation of its resolution 1820 (2008).

"The Security Council requests the Secretary-General to continue to submit an annual report to it on the implementation of resolution 1325 (2000). The Council further requests the Secretary-General to propose in his next annual report a strategic framework to guide the UN's implementation of the resolution in the next decade, which includes targets and indicators and takes account of relevant processes within the Secretariat. In this context, the Council requests the SecretaryGeneral to include recommendations for policy and institutional reforms in the UN that will facilitate improved response by the Organization to women and peace and security issues.

"The Security Council reiterates its request to Member States, international, regional and subregional organizations to take measures to increase the participation of women in conflict prevention, conflict resolution and peacebuilding, including in decision-making roles in post-conflict governance institutions, appointed and elected. The Council urges the Secretary-General to appoint more women as mediators and special representatives and envoys to pursue good offices on his behalf.

"The Security Council expresses its intention to convene a High-level Review in five years to assess progress at the global, regional and national levels in implementing resolution 1325, renew commitments and address obstacles and constraints that have emerged in the implementation of resolution 1325." 


\section{Anhang}

\section{Security Council}

Distr.: General

16 December 2010

\section{Resolution 1960 (2010)}

\section{Adopted by the Security Council at its 6453rd meeting, on 16 December 2010}

\section{The Security Council,}

Reaffirming its commitment to the continuing and full implementation, in a mutually reinforcing manner, of resolutions 1325 (2000), 1612 (2005), 1674 (2006), 1820 (2008), 1882 (2009), 1888 (2009), 1889 (2009) and 1894 (2009), and all relevant statements of its President,

Welcoming the report of the Secretary-General of 24 November 2010 (S/2010/604), but remaining deeply concerned over the slow progress on the issue of sexual violence in situations of armed conflict in particular against women and children, and noting as documented in the Secretary-General's report that sexual violence occurs in armed conflicts throughout the world,

Reiterating deep concern that despite its repeated condemnation of violence against women and children in situations of armed conflict, including sexual violence in situations of armed conflict, and despite its calls addressed to all parties to armed conflict for the cessation of such acts with immediate effect, such acts continue to occur, and in some situations have become systematic and widespread, reaching appalling levels of brutality,

Reiterating the necessity for all States and non-State parties to conflicts to comply fully with their obligations under applicable international law, including the prohibition on all forms of sexual violence,

Reiterating the need for civilian and military leaders, consistent with the principle of command responsibility, to demonstrate commitment and political will to prevent sexual violence and to combat impunity and enforce accountability, and that inaction can send a message that the incidence of sexual violence in conflicts is tolerated,

Recalling the responsibilities of States to end impunity and to prosecute those responsible for genocide, crimes against humanity, war crimes, and other egregious crimes perpetrated against civilians and, in this regard, noting with concern that only limited numbers of perpetrators of sexual violence have been brought to justice, while recognizing that in conflict and in post-conflict situations national justice systems may be significantly weakened, 
Welcoming the progress made in rendering operational the team of experts to assist national authorities to strengthen the rule of law in accordance with resolution 1888 (2009); reaffirming the importance of deploying it rapidly to situations of particular concern with respect to sexual violence in armed conflict, working through the United Nations presence on the ground and with the consent of the host government and in this regard, appreciating the voluntary contributions to support its work,

Recognizing that States bear the primary responsibility to respect and ensure the human rights of all persons within their territory and subject to their jurisdiction as provided for by international law,

Reaffirming that parties to armed conflict bear the primary responsibility to take all feasible steps to ensure the protection of civilians,

Recalling that international humanitarian law affords general protection to women and children as part of the civilian population during armed conflicts and special protection due to the fact that they can be placed particularly at risk,

Reaffirming that ending impunity is essential if a society in conflict or recovering from conflict is to come to terms with past abuses committed against civilians affected by armed conflict and to prevent future such abuses, drawing attention to the full range of justice and reconciliation mechanisms to be considered, including national, international and "mixed" criminal courts and tribunals and truth and reconciliation commissions, and noting that such mechanisms can promote not only individual responsibility for serious crimes, but also peace, truth, reconciliation and the rights of the victims,

Recalling the inclusion of a range of sexual violence offences in the Rome Statute of the International Criminal Court and the statutes of the ad hoc international criminal tribunals,

Reaffirming the importance for States, with the support of the international community, to increase access to health care, psychosocial support, legal assistance, and socio-economic reintegration services for victims of sexual violence, in particular in rural areas, and taking into account the specific needs of persons with disabilities,

Welcoming the proposals, conclusions and recommendations included in the report of the Special Committee on Peacekeeping Operations (A/64/19) on the need for adequate capabilities and clear and appropriate guidelines to enable peacekeeping missions to carry out all their mandated tasks, including prevention of and response to sexual violence; stressing the importance of ensuring engagement by senior mission leadership on protection of civilians, including the prevention of and response to instances of sexual violence in armed conflict, with a view to ensuring that all mission components and all levels of the chain of command are properly informed of and involved in the mission's mandate and their relevant responsibilities; welcoming progress made by the Secretary-General in developing operational tools for the implementation of protection of civilians mandates; and encouraging troop- and police-contributing countries to make full use of and provide feedback on these important materials,

Recognizing the efforts of the Secretary-General to address the underrepresentation of women in formal peace processes, the lack of mediators and 
ceasefire monitors with proper training in dealing with sexual violence, and the lack of women as Chief or Lead peace mediators in United Nations-sponsored peace talks; and encouraging further such efforts,

Welcoming the inclusion of women in peacekeeping missions in civil, military and police functions, recognizing that their presence may encourage women from local communities to report acts of sexual violence,

Having considered the report of the Secretary-General of 24 November 2010 (S/2010/604) and stressing that the present resolution does not seek to make any legal determination as to whether situations that are referred to in the SecretaryGeneral's report are or are not armed conflicts within the context of the Geneva Conventions and the Additional Protocols thereto, nor does it prejudge the legal status of non-State parties involved in these situations,

1. Reaffirms that sexual violence, when used or commissioned as a tactic of war or as a part of a widespread or systematic attack against civilian populations, can significantly exacerbate and prolong situations of armed conflict and may impede the restoration of international peace and security; affirms in this regard that effective steps to prevent and respond to such acts of sexual violence can significantly contribute to the maintenance of international peace and security; and expresses its readiness, when considering situations on the agenda of the Council, to take, where necessary, appropriate steps to address widespread or systematic sexual violence in situations of armed conflict;

2. Reiterates its demand for the complete cessation with immediate effect by all parties to armed conflict of all acts of sexual violence;

3. Encourages the Secretary-General to include in his annual reports submitted pursuant to resolutions 1820 (2008) and 1888 (2009) detailed information on parties to armed conflict that are credibly suspected of committing or being responsible for acts of rape or other forms of sexual violence, and to list in an annex to these annual reports the parties that are credibly suspected of committing or being responsible for patterns of rape and other forms of sexual violence in situations of armed conflict on the Security Council agenda; expresses its intention to use this list as a basis for more focused United Nations engagement with those parties, including, as appropriate, measures in accordance with the procedures of the relevant sanctions committees;

4. Requests the Secretary-General, in accordance with the present resolution and taking into account its specificity, to apply the listing and de-listing criteria for parties listed in his annual report on sexual violence in armed conflict consistent with paragraphs $175,176,178$, and 180 of his report A/64/742-S/2010/181;

5. Calls upon parties to armed conflict to make and implement specific and time-bound commitments to combat sexual violence, which should include, inter alia, issuance of clear orders through chains of command prohibiting sexual violence and the prohibition of sexual violence in Codes of Conduct, military field manuals, or equivalent; and further calls upon those parties to make and implement specific commitments on timely investigation of alleged abuses in order to hold perpetrators accountable;

6. Requests the Secretary-General to track and monitor implementation of these commitments by parties to armed conflict on the Security Council's agenda 
that engage in patterns of rape and other sexual violence, and regularly update the Council in relevant reports and briefings;

7. Reiterates its intention, when adopting or renewing targeted sanctions in situations of armed conflict, to consider including, where appropriate, designation criteria pertaining to acts of rape and other forms of sexual violence; and calls upon all peacekeeping and other relevant United Nations missions and United Nations entities, in particular the Working Group on Children and Armed Conflict, the Special Representative of the Secretary General for Children and Armed Conflict, and the Special Representative of the Secretary General on Sexual Violence in Conflict, to share with relevant United Nations Security Council Sanctions Committees, including through relevant United Nations Security Council Sanction Committees' monitoring groups and groups of experts, all pertinent information about sexual violence;

8. Requests the Secretary General to establish monitoring, analysis and reporting arrangements on conflict-related sexual violence, including rape in situations of armed conflict and post-conflict and other situations relevant to the implementation of resolution 1888 (2009), as appropriate, and taking into account the specificity of each country, that ensure a coherent and coordinated approach at the field-level, and encourages the Secretary-General to engage with United Nations actors, national institutions, civil society organizations, health-care service providers, and women's groups to enhance data collection and analysis of incidents, trends, and patterns of rape and other forms of sexual violence to assist the Council's consideration of appropriate actions, including targeted and graduated measures, while respecting fully the integrity and specificity of the monitoring and reporting mechanism implemented under Security Council resolutions 1612 (2005) and 1882 (2009) on children and armed conflict;

9. Requests the Secretary-General to continue to ensure full transparency, cooperation and coordination of efforts between the Special Representative of the Secretary-General for Children and Armed Conflict and the Special Representative of the Secretary-General on Sexual Violence in Conflict;

10. Welcomes the work of gender advisers; looks forward to the appointment of more women protection advisers to peacekeeping missions, in accordance with resolution 1888 (2009); notes their potential contribution in the framework of the monitoring, analysis, and reporting arrangements to be established pursuant to OP8 of the present resolution;

11. Welcomes the elaboration by the Secretary-General of scenario-based training materials on combating sexual violence for peacekeepers and encourages Member States to use them as a reference for the preparation and deployment of United Nations peacekeeping operations;

12. Underlines that, in order to carry out their mandate, missions must communicate effectively with local communities; and encourages the SecretaryGeneral to improve their capacity to do so;

13. Expresses its intention to give due consideration to sexual violence in mandate authorizations and renewals and to request the Secretary-General to include, as appropriate, gender expertise in technical assessment missions; 
14. Encourages the entities comprising United Nations Action Against Sexual Violence in Conflict, as well as other relevant parts of the United Nations system, to continue to support the work of the aforementioned Special Representative of the Secretary-General on Sexual Violence in Conflict and to enhance cooperation and information-sharing among all relevant stakeholders in order to reinforce coordination and avoid overlap at the headquarters and country levels and improve system-wide response;

15. Encourages Member States to deploy greater numbers of female military and police personnel to United Nations peacekeeping operations, and to provide all military and police personnel with adequate training on sexual and gender-based violence, inter alia, to carry out their responsibilities;

16. Requests the Secretary-General to continue and strengthen efforts to implement the policy of zero tolerance on sexual exploitation and abuse by United Nations peacekeeping and humanitarian personnel, and further requests the Secretary-General to continue to provide and deploy guidance on addressing sexual violence for predeployment and inductive training of military and police personnel, and to assist missions in developing situation-specific procedures to address sexual violence at the field level and to ensure that technical support is provided to troop and police contributing countries in order to include guidance for military and police personnel on addressing sexual violence in predeployment and induction training;

17. Invites the Special Representative on Sexual Violence in Conflict to continue to provide briefings on sexual violence, in accordance with resolution 1888 (2009);

18. Requests that the Secretary-General continue to submit annual reports to the Council on the implementation of Resolutions 1820 (2008) and 1888 (2009) and to submit his next report by December 2011 on the implementation of Resolutions 1820 (2008) and 1888 (2009) and the present resolution to include, inter alia:

(a) a detailed coordination and strategy plan on the timely and ethical collection of information;

(b) information on progress made in the implementation of the monitoring, analysis, and reporting arrangements mentioned in paragraph 8 ;

(c) detailed information on parties to armed conflict that are credibly suspected of committing or being responsible for acts of rape or other forms of sexual violence, and an annex with a list of parties that are credibly suspected of committing or being responsible for patterns of rape and other forms of sexual violence in situations of armed conflict on the Security Council agenda;

(d) updates on efforts by United Nations Mission focal points on sexual violence to work closely with Resident Coordinator/Humanitarian Coordinator (RC/HC), the United Nations Country Team, and, where appropriate, the Special Representative of the Secretary-General for Children and Armed Conflict and the Special Representative of the Secretary-General on Sexual Violence in Conflict and/or the Team of Experts, to address sexual violence;

19. Decides to remain actively seized of the matter. 


\section{Resolution 1882 (2009)}

\section{Adopted by the Security Council at its 6176th meeting, on 4 August 2009}

\section{The Security Council,}

Reaffirming its resolutions 1261 (1999) of 25 August 1999, 1314 (2000) of 11 August 2000, 1379 (2001) of 20 November 2001, 1460 (2003) of 30 January 2003, 1539 (2004) of 22 April 2004, and 1612 (2005) of 26 July 2005, and the Statements of its President on 24 July 2006 (S/PRST/2006/33), 28 November 2006 (S/PRST/2006/48), 12 February 2008 (S/PRST/2008/6), 17 July 2008 (S/PRST/2008/28) and 29 April 2009 (S/PRST/2009/9), which contribute to a comprehensive framework for addressing the protection of children affected by armed conflict,

Acknowledging that the implementation of its resolution 1612 (2005) has generated progress, resulting in the release and reintegration of children into their families and communities and in a more systematic dialogue between the United Nations country-level task forces and parties to the armed conflict on the implementation of time-bound action plans, while remaining deeply concerned over the lack of progress on the ground in some situations of concern, where parties to conflict continue to violate with impunity the relevant provisions of applicable international law relating to the rights and protection of children in armed conflict,

Stressing the primary role of national Governments in providing protection and relief to all children affected by armed conflicts,

Reiterating that all actions undertaken by United Nations entities within the framework of the monitoring and reporting mechanism must be designed to support and supplement, as appropriate, the protection and rehabilitation roles of national Governments,

Recalling the responsibilities of States to end impunity and to prosecute those responsible for genocide, crimes against humanity, war crimes and other egregious crimes perpetrated against children,

Welcoming the fact that several individuals who are alleged to have committed crimes against children in situations of armed conflict have been brought to justice by national justice systems and international justice mechanisms and mixed criminal courts and tribunals, 


\section{Anhang}

Convinced that the protection of children in armed conflict should be an important aspect of any comprehensive strategy to resolve conflict,

Calling on all parties to armed conflicts to comply strictly with the obligations applicable to them under international law for the protection of children in armed conflict, including those contained in the Convention on the Rights of the Child and its Optional Protocol on the involvement of Children in Armed Conflict, as well as the Geneva Conventions of 12th August 1949 and their Additional Protocols of 1977 ,

Reiterating its primary responsibility for the maintenance of international peace and security and, in this connection, its commitment to address the widespread impact of armed conflict on children,

Stressing its determination to ensure respect for its resolutions and other international obligations and applicable norms on the protection of children affected by armed conflict,

Having considered the report of the Secretary-General of 26 March 2009 (S/2009/158) and stressing that the present resolution does not seek to make any legal determination as to whether situations which are referred to in the SecretaryGeneral's report are or are not armed conflicts within the context of the Geneva Conventions and the Additional Protocols thereto, nor does it prejudge the legal status of the non-State parties involved in these situations,

Deeply concerned that children continue to account for a considerable number of casualties resulting from killing and maiming in armed conflicts including as a result of deliberate targeting, indiscriminate and excessive use of force, indiscriminate use of landmines, cluster munitions and other weapons and use of children as human shields and equally deeply concerned about the high incidence and appalling levels of brutality of rape and other forms of sexual violence committed against children, in the context of and associated with armed conflict including the use or commissioning of rape and other forms of sexual violence in some situations as a tactic of war,

1. Strongly condemns all violations of applicable international law involving the recruitment and use of children by parties to armed conflict as well as their re-recruitment, killing and maiming, rape and other sexual violence, abductions, attacks against schools or hospitals and denial of humanitarian access by parties to armed conflict and all other violations of international law committed against children in situations of armed conflict;

2. Reaffirms that the monitoring and reporting mechanism will continue to be implemented in situations listed in the annexes to the reports of the SecretaryGeneral on children and armed conflict in line with the principles set out in paragraph 2 of its resolution 1612 (2005) and that its establishment and implementation shall not prejudge or imply a decision by the Security Council as to whether or not to include a situation on its agenda;

3. Recalls paragraph 16 of its resolution 1379 (2001) and requests the Secretary-General also to include in the annexes to his reports on children and armed conflict those parties to armed conflict that engage, in contravention of applicable international law, in patterns of killing and maiming of children and/or rape and other sexual violence against children, in situations of armed conflict, 
bearing in mind all other violations and abuses against children, and notes that the present paragraph will apply to situations in accordance with the conditions set out in paragraph 16 of its resolution 1379 (2001);

4. Invites the Secretary-General through his Special Representative for Children and Armed Conflict to exchange appropriate information and maintain interaction from the earliest opportunity with the governments concerned regarding violations and abuses committed against children by parties which may be included in the annexes to his periodic report;

5. While noting that some parties to armed conflict have responded to its call upon them to prepare and implement concrete time-bound action plans to halt recruitment and use of children in violation of applicable international law;

(a) Reiterates its call on parties to armed conflict listed in the annexes of the Secretary-General's report on children and armed conflict that have not already done so to prepare and implement, without further delay, action plans to halt recruitment and use of children in violation of applicable international law;

(b) Calls upon those parties listed in the annexes of the Secretary-General's report on children and armed conflict that commit, in contravention of applicable international law, killing and maiming of children and/or rape and other sexual violence against children, in situations of armed conflict, to prepare concrete timebound action plans to halt those violations and abuses;

(c) Further calls upon all parties listed in the annexes to the SecretaryGeneral's report on children and armed conflict to address all other violations and abuses committed against children and undertake specific commitments and measures in this regard;

(d) Urges those parties listed in the annexes of the Secretary-General's report on children and armed conflict to implement the provisions contained in this paragraph in close cooperation with the Special Representative of the SecretaryGeneral for Children and Armed Conflict and the United Nations country-level task forces on monitoring and reporting;

6. In this context, encourages Member States to devise ways, in close consultations with the United Nations country-level task force on monitoring and reporting and United Nations country teams, to facilitate the development and implementation of time-bound action plans, and the review and monitoring by the United Nations country-level task force of obligations and commitments relating to the protection of children in armed conflict;

7. Reiterates its determination to ensure respect for its resolutions on children and armed conflict, and in this regard:

(a) Welcomes the sustained activity and recommendations of its Working Group on Children and Armed Conflict as called for in paragraph 8 of its resolution 1612 (2005), and invites it to continue reporting regularly to the Security Council;

(b) Requests enhanced communication between the Working Group and relevant Security Council Sanctions Committees, including through the exchange of pertinent information on violations and abuses committed against children in armed conflict; 


\section{Anhang}

(c) Reaffirms its intention to take action against persistent perpetrators in line with paragraph 9 of its resolution 1612 (2005);

8. Stresses the responsibility of the United Nations country-level task forces on monitoring and reporting and United Nations country teams, consistent with their respective mandates, to ensure effective follow-up to Security Council resolutions on children and armed conflict, to monitor and report progress to the SecretaryGeneral in close cooperation with his Special Representative for Children and Armed Conflict and ensure a coordinated response to issues related to children and armed conflict;

9. Requests the Secretary-General to include more systematically in his reports on children and armed conflict specific information regarding the implementation of the Working Group recommendations;

10. Reiterates its request to the Secretary-General to ensure that, in all his reports on country-specific situations, the matter of children and armed conflict is included as a specific aspect of the report, and expresses its intention to give its full attention to the information provided therein, including the implementation of relevant Security Council resolutions and of the recommendations of its Working Group on Children and Armed Conflict, when dealing with those situations on its agenda;

11. Welcomes the efforts of the Department of Peacekeeping Operations in mainstreaming child protection into peacekeeping missions, in line with that Department's recently adopted Child Protection Policy directive, and encourages the deployment of Child Protection Advisers to peacekeeping operations, as well as into relevant peacebuilding and political missions, and decides to continue the inclusion of specific provisions for the protection of children in such mandates;

12. Requests Member States, United Nations peacekeeping, peacebuilding and political missions and United Nations country teams, within their respective mandates and in close cooperation with governments of the concerned countries, to establish appropriate strategies and coordination mechanisms for information exchange and cooperation on child protection concerns, in particular cross-border issues, bearing in mind relevant conclusions by the Security Council Working Group on Children and Armed Conflict and paragraph 2 (d) of its resolution 1612 (2005);

13. Stresses that effective disarmament, demobilization and reintegration programmes for children, building on best practices identified by UNICEF and other relevant child protection actors, are crucial for the well-being of all children who, in contravention of applicable international law, have been recruited or used by armed forces and groups, and are a critical factor for durable peace and security, and urges national Governments and donors to ensure that these community-based programmes receive timely, sustained and adequate resources and funding;

14. Also stresses the importance of timely, sustained and adequate resources and funding for effective welfare programmes for all children affected by armed conflict;

15. Calls upon Member States, United Nations entities, including the Peacebuilding Commission and other parties concerned to ensure that the protection, rights, well-being and empowerment of children affected by armed conflict are integrated into all peace processes and that post-conflict recovery and reconstruction 
planning, programmes and strategies prioritize issues concerning children affected by armed conflict;

16. Calls upon concerned Member States to take decisive and immediate action against persistent perpetrators of violations and abuses committed against children in situations of armed conflict, and further calls upon them to bring to justice those responsible for such violations that are prohibited under applicable international law, including with regard to recruitment and use of children, killing and maiming and rape and other sexual violence, through national justice systems, and where applicable, international justice mechanisms and mixed criminal courts and tribunals, with a view to ending impunity for those committing crimes against children;

17. Requests the Secretary-General to continue to take the necessary measures including, where applicable, to bring the monitoring and reporting mechanism to its full capacity, to allow for prompt advocacy and effective response to all violations and abuses committed against children and to ensure that information collected and communicated by the mechanism is accurate, objective, reliable and verifiable;

18. Requests the Secretary-General to provide administrative and substantive support for the Security Council Working Group on Children and Armed Conflict taking into consideration its current workload and the need to strengthen its capacities and institutional memory;

19. Requests the Secretary-General to submit a report by May 2010 on the implementation of its resolutions and presidential statements on children and armed conflict, including the present resolution, which would include, inter alia:

(a) Annexed lists of parties in situations of armed conflict on the agenda of the Security Council or in other situations of concern, in accordance with paragraph 3 of the present resolution;

(b) Information on measures undertaken by parties listed in the annexes to end all violations and abuses committed against children in armed conflict;

(c) Information of progress made in the implementation of the monitoring and reporting mechanism established in its resolution 1612 (2005);

(d) Information on the criteria and procedures used for listing and de-listing parties to armed conflict in the annexes to his periodic reports, bearing in mind the views expressed by all the members of the Working Group during informal briefings to be held before the end of 2009 ;

20. Decides to remain actively seized of this matter. 


\section{Anhang}

\section{Security Council}

Distr.: General

16 June 2010

Original: English

\section{Statement by the President of the Security Council}

At the 6341st meeting of the Security Council, held on 16 June 2010, in connection with the Council's consideration of the item entitled "Children and armed conflict", the President of the Security Council made the following statement on behalf of the Council:

"The Security Council takes note with appreciation of the 9th report of the Secretary-General on children and armed conflict (S/2010/181) and the recommendations contained therein as well as the positive developments referred to in the report, and notes the continuing challenges in the implementation of its resolution 1612 (2005), 1882 (2009) and other relevant resolutions reflected therein.

"The Security Council reiterates its equally strong condemnation of all violations of applicable international law involving the recruitment and use of children by parties to armed conflict as well as their re-recruitment, killing and maiming, rape and other sexual violence, abductions, attacks against schools or hospitals and denial of humanitarian access by parties to armed conflict. The Council condemns all other violations of international law, including international humanitarian law, human rights law and refugee law, committed against children in situations of armed conflict. The Council demands that all relevant parties immediately put an end to such practices and take special measures to protect children.

"The Security Council expresses deep concern about the growing number of attacks or threats of attacks in contravention of applicable international law against schools and educational facilities, and teachers and pupils, in particular the specific targeting of girls and in this regard calls upon all parties to armed conflict to cease immediately these violations of international humanitarian law.

"The Security Council welcomes the steps taken by the SecretaryGeneral in the implementation of resolution 1882 (2009) by including in the annexes of his report those parties to armed conflict that engage, in contravention of applicable international law, in patterns of killing and maiming of children and/or rape and other sexual violence, in situations of armed conflict.

"The Security Council calls upon the Secretary-General to redouble his efforts to ensure strengthening of the Monitoring and Reporting Mechanism's 
capacities with a view to the implementation of resolution 1882 (2009) to allow for prompt advocacy and effective response to all violations and abuses committed against children, inter alia, by ensuring that all relevant UN agencies actively help to collect accurate, objective, reliable and verifiable information on acts of rape and other sexual violence committed against children, and also by ensuring synergies and avoiding overlap among relevant UN entities, at the headquarters and country levels as requested by resolutions 1882 (2009) and 1888 (2009).

"The Security Council reaffirms its decision in OP11 of its resolution 1882 (2009) to continue to include specific provisions for the protection of children in the mandates of all relevant United Nations peacekeeping, peacebuilding and political missions, encourages the deployment of Child Protection Advisers to such missions and calls upon the Secretary-General to ensure that such advisors are recruited and deployed in line with the Council's relevant country-specific resolutions and the Department of Peacekeeping Operations (DPKO) Policy Directive on Mainstreaming the Protection Rights and Wellbeing of Children Affected by Armed Conflict. It further underscores the importance of training on child rights and child protection for all personnel involved in United Nations peacekeeping, peace building and political missions and in this regard welcomes the ongoing efforts by DPKO in developing the policy implementation plan, including training programmes and materials.

"The Security Council welcomes the progress made in preventing and responding to violations and abuses committed against children especially with regard to the signing of action plans by some parties as mentioned in the 9th report of the Secretary-General (S/2010/181).

"The Security Council reiterates its call on parties to armed conflict listed in the annexes of the Secretary-General's report on children and armed conflict that have not already done so to prepare and implement, without further delay, action plans to halt recruitment and use of children, patterns of killing and maiming of children and/or rape and other sexual violence against children, in violation of applicable international law, in situations of armed conflict.

"The Security Council also reiterates its call to all parties listed in the annexes to the Secretary-General's report on children and armed conflict to address all other violations and abuses committed against children and undertake specific commitments and measures in this regard.

"The Security Council expresses deep concern that certain parties persist in committing violations and abuses against children, and expresses its readiness to adopt targeted and graduated measures against persistent perpetrators taking in to account the relevant provisions of its resolutions 1539 (2004), 1612 (2005) and 1882 (2009). To this end, the Council invites:

(a) Its Working Group on children and armed conflict to exchange pertinent information with relevant Sanctions Committees, in particular through communication of the Working Group's relevant recommendations.

(b) Its relevant Sanctions Committees to consider inviting more regularly the Special Representative of the Secretary-General for children and 


\section{Anhang}

armed conflict to brief them on specific information contained in the Secretary General's reports.

(c) The Special Representative of the Secretary-General for children and armed conflict to share specific information contained in the SecretaryGeneral's reports with relevant Sanctions Committees expert groups.

"The Security Council expresses its intention, when establishing or renewing the mandate of relevant Sanctions Committees, to consider provisions pertaining to parties that are in violation of applicable international law relating to the rights and protection of children in armed conflict.

"The Security Council expresses its readiness to consider specific recommendations from its Working Group on Children and Armed Conflict on violations and abuses committed against children by parties listed in the annexes of the Secretary-General's reports, whenever they occur, with a view to considering action on them, without prejudging or implying a decision by the Security Council as to whether or not to include a situation in its agenda.

"The Security Council calls upon concerned Member States to take decisive and immediate action against persistent perpetrators of violations and abuses committed against children in situations of armed conflict, and further calls upon them to bring to justice those responsible for such violations that are prohibited under applicable international law, including with regard to recruitment and use of children, killing and maiming and rape and other sexual violence, through national justice systems, and where applicable, international justice mechanisms and mixed criminal courts and tribunals, with a view to ending impunity for those committing crimes against children.

"Given the regional dimensions of some armed conflicts referred to in the Secretary- General's report, the Security Council reiterates its request that United Nations peacekeeping, peacebuilding and political missions as well as United Nations country teams, within their respective mandates and in close cooperation with governments of the concerned countries, develop appropriate strategies and coordination mechanisms for information exchange and cooperation on cross-border child protection concerns.

"The Security Council welcomes the overall work of the Special Representative of the Secretary-General for children and armed conflict and highlights the importance of her field visits in enhancing dialogue with concerned governments and parties to the conflict, including by negotiating action plans, securing commitments, advocating for appropriate response mechanisms and ensuring appropriate attention and follow up to the Security Council Working Group on Children and Armed Conflict conclusions and recommendations.

"The Security Council also welcomes the efforts undertaken by UNICEF in carrying out its mandate on child protection by supporting the overall development and implementation of the Monitoring and Reporting Mechanism (MRM) and ensuring appropriate response to children in armed conflict, and encourages it to continue to follow up, through the MRM Country Task Forces, on relevant conclusions and recommendations of the Security Council Working Group on Children and Armed Conflict. 
"The Security Council welcomes the sustained activity of its Working Group on Children and Armed Conflict and stresses the importance of continuing to adopt timely conclusions and recommendations in line with resolutions 1612 (2005) and 1882 (2009). Furthermore, the Council invites the Working Group to fully implement its toolkit (S/2006/724), inter alia, by carrying out a country-specific visit within one year, to examine a situation referred to in the annexes of the Secretary-General's report in order to better fulfil its mandate and enhance its capacity to protect children affected by armed conflict.

"Recalling previous Presidential statements on children and armed conflict as well as paragraph 18 of resolution 1882 (2009), the Security Council reiterates its request to the Secretary-General to provide administrative and substantive support for its Working Group on children and armed conflict and further requests the Secretary-General to take action on this subject within one month of this date.

"The Security Council requests the Secretary-General to submit a report by May 2011 on the implementation of its resolutions and Presidential statements on children and armed conflict, including the present statement." 


\section{Anhang}

\section{Resolution 1904 (2009)}

\section{Adopted by the Security Council at its 6247th meeting, on 17 December 2009}

\section{The Security Council,}

Recalling its resolutions 1267 (1999), 1333 (2000), 1363 (2001), 1373 (2001), 1390 (2002), 1452 (2002), 1455 (2003), 1526 (2004), 1566 (2004), 1617 (2005), 1624 (2005), 1699 (2006), 1730 (2006), 1735 (2006), and 1822 (2008), and the relevant statements of its President,

Reaffirming that terrorism in all its forms and manifestations constitutes one of the most serious threats to peace and security and that any acts of terrorism are criminal and unjustifiable regardless of their motivations, whenever and by whomsoever committed, and reiterating its unequivocal condemnation of Al-Qaida, Usama bin Laden, the Taliban and other individuals, groups, undertakings and entities associated with them, for ongoing and multiple criminal terrorist acts aimed at causing the deaths of innocent civilians and other victims, destruction of property and greatly undermining stability,

Reaffirming the need to combat by all means, in accordance with the Charter of the United Nations and international law, including applicable international human rights, refugee and humanitarian law, threats to international peace and security caused by terrorist acts, stressing in this regard the important role the United Nations plays in leading and coordinating this effort,

Expressing concern at the increase in incidents of kidnapping and hostagetaking by individuals, groups, undertakings and entities associated with Al-Qaida, Usama bin Laden or the Taliban with the aim of raising funds, or gaining political concessions,

Reiterating its support for the fight against illicit production and trafficking of drugs from, and chemical precursors to Afghanistan, in neighbouring countries, countries on trafficking routes, drug destination countries and precursors producing countries,

Stressing that terrorism can only be defeated by a sustained and comprehensive approach involving the active participation and collaboration of all States, and international and regional organizations to impede, impair, isolate and incapacitate the terrorist threat, 
Emphasizing that sanctions are an important tool under the Charter of the United Nations in the maintenance and restoration of international peace and security, and stressing in this regard the need for robust implementation of the measures in paragraph 1 of this resolution as a significant tool in combating terrorist activity,

Urging all Member States to participate actively in maintaining and updating the list created pursuant to resolutions 1267 (1999) and 1333 (2000) ("the Consolidated List") by contributing additional information pertinent to current listings, submitting delisting requests when appropriate, and by identifying and nominating for listing additional individuals, groups, undertakings and entities which should be subject to the measures referred to in paragraph 1 of this resolution,

Taking note of challenges, both legal and otherwise, to the measures implemented by Member States under paragraph 1 of this resolution, welcoming improvements to the Committee's procedures and the quality of the Consolidated List, and expressing its intent to continue efforts to ensure that procedures are fair and clear,

Reiterating that the measures referred to in paragraph 1 of this resolution are preventative in nature and are not reliant upon criminal standards set out under national law,

Recalling the adoption by the General Assembly of the United Nations Global Counter-Terrorism Strategy (A/60/288) of 8 September 2006 and the creation of the Counter-Terrorism Implementation Task Force (CTITF) to ensure overall coordination and coherence in the counter-terrorism efforts of the United Nations system,

Welcoming the continuing cooperation between the Committee and INTERPOL, the United Nations Office on Drugs and Crime, in particular on technical assistance and capacity building, and all other United Nations bodies, and encouraging further engagement with the Counter-Terrorism Implementation Task Force (CTITF) to ensure overall coordination and coherence in the counterterrorism efforts of the United Nations system,

Noting with concern the continued threat posed to international peace and security ten years after the adoption of resolution 1267 (1999) by Al-Qaida, Usama bin Laden and the Taliban, and other individuals, groups, undertakings and entities associated with them, and reaffirming its resolve to address all aspects of that threat,

Acting under Chapter VII of the Charter of the United Nations,

\section{Measures}

1. Decides that all States shall take the measures as previously imposed by paragraph 4 (b) of resolution 1267 (1999), paragraph 8 (c) of resolution 1333 (2000), and paragraphs 1 and 2 of resolution 1390 (2002), with respect to Al-Qaida, Usama bin Laden and the Taliban, and other individuals, groups, undertakings and entities associated with them, as referred to in the list created pursuant to resolutions 1267 (1999) and 1333 (2000) ("the Consolidated List");

(a) Freeze without delay the funds and other financial assets or economic resources of these individuals, groups, undertakings and entities, including funds derived from property owned or controlled directly or indirectly, by them or by 


\section{Anhang}

persons acting on their behalf or at their direction, and ensure that neither these nor any other funds, financial assets or economic resources are made available, directly or indirectly for such persons' benefit, by their nationals or by persons within their territory;

(b) Prevent the entry into or transit through their territories of these individuals, provided that nothing in this paragraph shall oblige any State to deny entry or require the departure from its territories of its own nationals and this paragraph shall not apply where entry or transit is necessary for the fulfilment of a judicial process or the Committee determines on a case-by-case basis only that entry or transit is justified;

(c) Prevent the direct or indirect supply, sale, or transfer to these individuals, groups, undertakings and entities from their territories or by their nationals outside their territories, or using their flag vessels or aircraft, of arms and related materiel of all types including weapons and ammunition, military vehicles and equipment, paramilitary equipment, and spare parts for the aforementioned, and technical advice, assistance, or training related to military activities;

2. Reaffirms that acts or activities indicating that an individual, group, undertaking, or entity is associated with Al-Qaida, Usama bin Laden or the Taliban include:

(a) participating in the financing, planning, facilitating, preparing, or perpetrating of acts or activities by, in conjunction with, under the name of, on behalf of, or in support of;

(b) supplying, selling or transferring arms and related materiel to;

(c) recruiting for; or

(d) otherwise supporting acts or activities of Al-Qaida, Usama bin Laden or the Taliban, or any cell, affiliate, splinter group or derivative thereof;

3. Further reaffirms that any undertaking or entity owned or controlled, directly or indirectly, by, or otherwise supporting, such an individual, group, undertaking or entity associated with Al-Qaida, Usama bin Laden or the Taliban shall be eligible for designation;

4. Confirms that the requirements in paragraph 1(a) above apply to financial and economic resources of every kind, including but not limited to those used for the provision of Internet hosting or related services, used for the support of Al-Qaida, Usama bin Laden, or the Taliban and other individuals, groups, undertakings, or entities associated with them;

5. Confirms further that the requirements in paragraph 1(a) above shall also apply to the payment of ransoms to individuals, groups, undertakings or entities on the Consolidated List;

6. Decides that Member States may permit the addition to accounts frozen pursuant to the provisions of paragraph 1 above of any payment in favour of listed individuals, groups, undertakings or entities, provided that any such payments continue to be subject to the provisions in paragraph 1 above and are frozen;

7. Encourages Member States to make use of the provisions regarding available exemptions to the measures in paragraph 1(a) above, set out in paragraphs 1 
and 2 of resolution 1452 (2002), as amended by resolution 1735 (2006), and directs the Committee to review the procedures for exemptions as set out in the Committee's guidelines to facilitate their use by Member States and to continue to ensure that humanitarian exemptions are granted expeditiously and transparently;

\section{Listing}

8. Encourages all Member States to submit to the Committee for inclusion on the Consolidated List names of individuals, groups, undertakings and entities participating, by any means, in the financing or support of acts or activities of Al-Qaida, Usama bin Laden or the Taliban, and other individuals, groups, undertakings and entities associated with them, as described in paragraph 2 of resolution 1617 (2005) and reaffirmed in paragraph 2 above, and further encourages Member States to appoint a national contact point concerning entries on the Consolidated List;

9. Notes that such means of financing or support include but are not limited to the use of proceeds derived from illicit cultivation, production and trafficking of narcotic drugs originating particularly in Afghanistan, and their precursors;

10. Reiterates its call for continued cooperation between the Committee and the Government of Afghanistan and the United Nations Assistance Mission in Afghanistan (UNAMA), including by identifying individuals and entities participating in the financing or support of acts or activities of Al-Qaida and the Taliban as described in paragraph 30 of resolution 1806 (2008);

11. Reaffirms that, when proposing names to the Committee for inclusion on the Consolidated List, Member States shall act in accordance with paragraph 5 of resolution 1735 (2006) and paragraph 12 of resolution 1822 (2008) and provide a detailed statement of case, and decides further that the statement of case shall be releasable, upon request, except for the parts a Member State identifies as being confidential to the Committee, and may be used to develop the narrative summary of reasons for listing described in paragraph 14 below;

12. Encourages Member States proposing a new designation, as well as Member States that have proposed names for inclusion on the Consolidated List before the adoption of this resolution, to specify whether the Committee may make known, upon request from a Member State, the Member State's status as a designating State;

13. Calls upon Member States, when proposing names to the Committee for inclusion on the Consolidated List to use the new standard form for listing, once it is adopted and placed on the Committee's website, and requests that they provide the Committee with as much relevant information as possible on the proposed name, in particular sufficient identifying information to allow for the accurate and positive identification of individuals, groups, undertakings and entities, and directs the Committee to update, as necessary, the standard form for listing in accordance with the provisions of this resolution;

14. Directs the Committee, with the assistance of the Monitoring Team and in coordination with the relevant designating States, to make accessible on the Committee's website, at the same time a name is added to the Consolidated List, a narrative summary of reasons for listing for the corresponding entry or entries, and further directs the Committee, with the assistance of the Monitoring Team and in 


\section{Anhang}

coordination with the relevant designating States, to continue its efforts to make accessible on the Committee's website narrative summaries of reasons for listing for entries that were added to the Consolidated List before the date of adoption of resolution 1822 (2008);

15. Encourages Member States and relevant international organizations to inform the Committee of any relevant court decisions and proceedings so that the Committee can consider them when it reviews a corresponding listing or updates a narrative summary of reasons for listing;

16. Calls upon all members of the Committee and the Monitoring Team to share with the Committee any information they may have available regarding a listing request from a Member State so that this information may help inform the Committee's decision on designation and provide additional material for the narrative summary of reasons for listing described in paragraph 14;

17. Directs the Committee to amend its Guidelines to extend the period of time for members of the Committee to verify that names proposed for listing merit inclusion in the Consolidated List and include adequate identifying information to ensure full implementation of the measures, with exceptions, at the Committee chair's discretion, for emergency and time-sensitive listings, and notes that listing requests may be placed on the Committee's agenda upon request of a Committee member;

18. Decides that the Secretariat shall, after publication but within three working days after a name is added to the Consolidated List, notify the Permanent Mission of the country or countries where the individual or entity is believed to be located and, in the case of individuals, the country of which the person is a national (to the extent this information is known), in accordance with paragraph 10 of resolution 1735 (2006), and requests the Secretariat to publish on the Committee's website all relevant publicly releasable information, including the narrative summary of reasons for listing, immediately after a name is added to the Consolidated List;

19. Reaffirms further the provisions in paragraph 17 of resolution 1822 (2008) regarding the requirement that Member States take all possible measures, in accordance with their domestic laws and practices, to notify or inform in a timely manner the listed individual or entity of the designation and to include with this notification the narrative summary of reasons for listing, a description of the effects of designation, as provided in the relevant resolutions, the Committee's procedures for considering delisting requests, including the possibility of submitting such a request to the Ombudsperson in accordance with paragraphs 20 and 21 and annex II of this resolution, and the provisions of resolution 1452 (2002) regarding available exemptions;

\section{Delisting/Ombudsperson}

20. Decides that, when considering delisting requests, the Committee shall be assisted by an Office of the Ombudsperson, to be established for an initial period of 18 months from the date of adoption of this resolution, and requests the Secretary-General, in close consultation with the Committee, to appoint an eminent individual of high moral character, impartiality and integrity with high qualifications and experience in relevant fields, such as legal, human rights, 
counter-terrorism and sanctions, to be Ombudsperson, with the mandate outlined in annex II of this resolution, and further decides that the Ombudsperson shall perform these tasks in an independent and impartial manner and shall neither seek nor receive instructions from any government;

21. Decides that, after the appointment of the Ombudsperson, the Office of the Ombudsperson shall receive requests from individuals and entities seeking to be removed from the Consolidated List, in accordance with the procedures outlined in annex II of this resolution, and that, after the appointment of the Ombudsperson, the Focal Point mechanism established in resolution 1730 (2006) shall no longer receive such requests, and notes that the Focal Point shall continue to receive requests from individuals and entities seeking to be removed from other sanctions lists;

22. Directs the Committee to continue to work, in accordance with its guidelines, to consider delisting requests of Member States for the removal from the Consolidated List of members and/or associates of Al-Qaida, Usama bin Laden, or the Taliban who no longer meet the criteria established in the relevant resolutions, which shall be placed on the Committee's agenda upon request of a member of the Committee;

23. Encourages States to submit delisting requests for individuals that are officially confirmed to be dead, particularly where no assets are identified, and for entities that have ceased to exist, while at the same time taking all reasonable measures to ensure that the assets that had belonged to these individuals or entities have not been or will not be transferred or distributed to other entities or individuals on the Consolidated List;

24. Encourages Member States, when unfreezing the assets of a deceased individual or defunct entity as a result of a delisting, to recall the obligations set forth in resolution 1373 (2001) and, particularly, to prevent unfrozen assets from being used for terrorist purposes;

25. Encourages the Committee to give due consideration to the opinions of designating State(s), and State(s) of residence, nationality or incorporation when considering delisting requests, and calls on Committee members to make every effort to provide their reasons for objecting to such delisting requests;

26. Requests the Monitoring Team, upon conclusion of the review pursuant to paragraph 25 of resolution 1822 (2008), to circulate to the Committee every six months a list of individuals on the Consolidated List who are reportedly deceased, along with an assessment of relevant information such as the certification of death, and to the extent possible, the status and location of frozen assets and the names of any individuals or entities who would be in a position to receive any unfrozen assets, directs the Committee to review these listings to decide whether they remain appropriate, and encourages the Committee to remove listings of deceased individuals where credible information regarding death is available;

27. Decides that the Secretariat shall, within three working days after a name is removed from the Consolidated List, notify the Permanent Mission of the country or countries where the individual or entity is believed to be located and, in the case of individuals, the country of which the person is a national (to the extent this information is known), and demands that States receiving such notification take measures, in accordance with their domestic laws and practices, to notify or inform the concerned individual or entity of the delisting in a timely manner; 


\section{Anhang}

\section{Review and maintenance of the Consolidated List}

28. Encourages all Member States, in particular designating States and States of residence or nationality, to submit to the Committee additional identifying and other information, along with supporting documentation, on listed individuals, groups, undertakings and entities, including updates on the operating status of listed entities, groups and undertakings, the movement, incarceration or death of listed individuals and other significant events, as such information becomes available;

29. Welcomes the significant progress made by the Committee in its review of all names on the Consolidated List pursuant to paragraph 25 of resolution 1822 (2008), directs the Committee to complete this review by 30 June 2010, and requests that all States concerned respond to requests from the Committee for information relevant to this review no later than 1 March 2010;

30. Requests the Monitoring Team to submit a report to the Committee by 30 July 2010 on the outcome of the review described in paragraph 25 of resolution 1822 (2008) and the efforts made by the Committee, Member States and the Monitoring Team to conduct the review;

31. Requests the Monitoring Team, upon conclusion of the review described in paragraph 25 of resolution 1822 (2008), to circulate to the Committee annually a list of individuals and entities on the Consolidated List whose entries lack identifiers necessary to ensure effective implementation of the measures imposed upon them, and directs the Committee to review these listings to decide whether they remain appropriate;

32. Further directs the Committee, upon completion of the review described in paragraph 25 of resolution 1822 (2008), to conduct an annual review of all names on the Consolidated List that have not been reviewed in three or more years, in which the relevant names are circulated to the designating States and States of residence and/or citizenship, where known, pursuant to the procedures set forth in the Committee guidelines, in order to ensure the Consolidated List is as updated and accurate as possible and to confirm that listing remains appropriate, and notes that the Committee's consideration of a delisting request after the date of adoption of this resolution, pursuant to the procedures set out in annex II of this resolution, should be considered equivalent to a review of that listing;

\section{Measures implementation}

33. Reiterates the importance of all States identifying, and if necessary introducing, adequate procedures to implement fully all aspects of the measures described in paragraph 1 above;

34. Encourages the Committee to continue to ensure that fair and clear procedures exist for placing individuals and entities on the Consolidated List and for removing them as well as for granting humanitarian exemptions, and directs the Committee to keep its guidelines under active review in support of these objectives;

35. Directs the Committee, as a matter of priority, to review its guidelines with respect to the provisions of this resolution, in particular paragraphs $7,13,14$, $17,18,22,23,34$, and 41; 
36. Encourages Member States and relevant international organizations to send representatives to meet the Committee for more in-depth discussion of relevant issues and welcomes voluntary briefings from interested Member States on their efforts to implement the measures referred to in paragraph 1 above, including particular challenges that hinder full implementation of the measures;

37. Requests the Committee to report to the Council on its findings regarding Member States' implementation efforts, and identify and recommend steps necessary to improve implementation;

38. Directs the Committee to identify possible cases of non-compliance with the measures pursuant to paragraph 1 above and to determine the appropriate course of action on each case, and requests the Chairman, in periodic reports to the Council pursuant to paragraph 46 below, to provide progress reports on the Committee's work on this issue;

39. Urges all Member States, in their implementation of the measures set out in paragraph 1 above, to ensure that fraudulent, counterfeit, stolen and lost passports and other travel documents are invalidated and removed from circulation, in accordance with domestic laws and practices, as soon as possible, and to share information on those documents with other Member States through the INTERPOL database;

40. Encourages Member States to share, in accordance with their domestic laws and practices, with the private sector information in their national databases related to fraudulent, counterfeit, stolen and lost identity or travel documents pertaining to their own jurisdictions, and, if a listed party is found to be using a false identity including to secure credit or fraudulent travel documents, to provide the Committee with information in this regard;

41. Directs the Committee to amend its guidelines to ensure that no matter is left pending before the Committee for a period longer than six months, unless the Committee determines on a case-by-case basis that extraordinary circumstances require additional time for consideration, and further directs any Committee member that has requested more time to consider a proposal to provide updates after three months of their progress in resolving all pending matters;

42. Directs the Committee to conduct a comprehensive review of all issues pending before the Committee as of the date of adoption of this resolution, and further urges the Committee and its members to resolve all such pending issues, to the extent possible, by 31 December 2010;

\section{Coordination and outreach}

43. Reiterates the need to enhance ongoing cooperation among the Committee, the Counter Terrorism Committee (CTC) and the Committee established pursuant to resolution 1540 (2004), as well as their respective groups of experts, including through, as appropriate, enhanced information-sharing, coordination on visits to countries within their respective mandates, on facilitating and monitoring technical assistance, on relations with international and regional organizations and agencies and on other issues of relevance to all three committees, expresses its intention to provide guidance to the committees on areas of common interest in order better to coordinate their efforts and facilitate such cooperation, and requests 


\section{Anhang}

the Secretary-General to make the necessary arrangements for the groups to be co-located as soon as possible;

44. Encourages the Monitoring Team and the United Nations Office on Drugs and Crime, to continue their joint activities, in cooperation with CTED and 1540 Committee experts to assist Member States in their efforts to comply with their obligations under the relevant resolutions, including through organizing regional and subregional workshops;

45. Requests the Committee to consider, where and when appropriate, visits to selected countries by the Chairman and/or Committee members to enhance the full and effective implementation of the measures referred to in paragraph 1 above, with a view to encouraging States to comply fully with this resolution and resolutions 1267 (1999), 1333 (2000), 1390 (2002), 1455 (2003), 1526 (2004), 1617 (2005), 1735 (2006) and 1822 (2008);

46. Requests the Committee to report orally, through its Chairman, at least every 180 days to the Council on the state of the overall work of the Committee and the Monitoring Team, and, as appropriate, in conjunction with the reports by the Chairmen of CTC and the Committee established pursuant to resolution 1540 (2004), including briefings for all interested Member States;

\section{Monitoring Team}

47. Decides, in order to assist the Committee in fulfilling its mandate, as well as to support the Ombudsperson, to extend the mandate of the current New York-based Monitoring Team, established pursuant to paragraph 7 of resolution 1526 (2004), for a further period of 18 months, under the direction of the Committee with the responsibilities outlined in annex 1, and requests the Secretary-General to make the necessary arrangements to this effect;

\section{Reviews}

48. Decides to review the measures described in paragraph 1 above with a view to their possible further strengthening in 18 months, or sooner if necessary;

49. Decides to remain actively seized of the matter. 


\section{Resolution 1966 (2010)}

\section{Adopted by the Security Council at its 6463rd meeting, on 22 December 2010}

\section{The Security Council,}

Recalling Security Council resolution 827 (1993) of 25 May 1993, which established the International Tribunal for the former Yugoslavia ("ICTY"), and resolution 955 (1994) of 8 November 1994, which established the International Criminal Tribunal for Rwanda ("ICTR"), and all subsequent relevant resolutions,

Recalling in particular Security Council resolutions 1503 (2003) of 28 August 2003 and 1534 (2004) of 26 March 2004, which called on the Tribunals to take all possible measures to complete investigations by the end of 2004 , to complete all trial activities at first instance by the end of 2008, and to complete all work in 2010 ("completion strategy"), and noting that those envisaged dates have not been met,

Acknowledging the considerable contribution the Tribunals have made to international criminal justice and accountability for serious international crimes, and the re-establishment of the rule of law in the countries of the former Yugoslavia and in Rwanda,

Recalling that the Tribunals were established in the particular circumstances of the former Yugoslavia and Rwanda as ad hoc measures contributing to the restoration and maintenance of peace,

Reaffirming its determination to combat impunity for those responsible for serious violations of international humanitarian law and the necessity that all persons indicted by the ICTY and ICTR are brought to justice,

Recalling the statement of the President of the Security Council of 19 December 2008 (S/PRST/2008/47), and reaffirming the need to establish an ad hoc mechanism to carry out a number of essential functions of the Tribunals, including the trial of fugitives who are among the most senior leaders suspected of being most responsible for crimes, after the closure of the Tribunals,

Emphasizing that, in view of the substantially reduced nature of the residual functions, the international residual mechanism should be a small, temporary and efficient structure, whose functions and size will diminish over time, with a small number of staff commensurate with its reduced functions, 


\section{Anhang}

Welcoming the Report of the Secretary-General (S/2009/258) on the administrative and budgetary aspects of the options for possible locations for the archives of the International Tribunal for the former Yugoslavia and the International Criminal Tribunal for Rwanda and the seat of the residual mechanism(s) for the Tribunals,

Acting under Chapter VII of the Charter of the United Nations,

1. Decides to establish the International Residual Mechanism for Criminal Tribunals ("the Mechanism") with two branches, which shall commence functioning on 1 July 2012 (branch for the ICTR) and 1 July 2013 (branch for the ICTY), respectively ("commencement dates"), and to this end decides to adopt the Statute of the Mechanism in Annex 1 to this resolution;

2. Decides that the provisions of this resolution and the Statutes of the Mechanism and of the ICTY and ICTR shall be subject to the transitional arrangements set out in Annex 2 to this resolution;

3. Requests the ICTY and the ICTR to take all possible measures to expeditiously complete all their remaining work as provided by this resolution no later than 31 December 2014, to prepare their closure and to ensure a smooth transition to the Mechanism, including through advance teams in each of the Tribunals;

4. Decides that, as of the commencement date of each branch referred to in paragraph 1, the Mechanism shall continue the jurisdiction, rights and obligations and essential functions of the ICTY and the ICTR, respectively, subject to the provisions of this resolution and the Statute of the Mechanism, and all contracts and international agreements concluded by the United Nations in relation to the ICTY and the ICTR, and still in force as of the relevant commencement date, shall continue in force mutatis mutandis in relation to the Mechanism;

5. Requests the Secretary-General to submit at the earliest possible date, but no later than 30 June 2011, draft Rules of Procedure and Evidence of the Mechanism, which shall be based on the Tribunals' Rules of Procedure and Evidence subject to the provisions of this resolution and the Statute of the Mechanism, for consideration and adoption by the judges of the Mechanism;

6. Decides that the Rules of Procedure and Evidence of the Mechanism and any amendments thereto shall take effect upon adoption by the judges of the Mechanism unless the Security Council decides otherwise;

7. Decides that the determination of the seats of the branches of the Mechanism is subject to the conclusion of appropriate arrangements between the United Nations and the host countries of the branches of the Mechanism acceptable to the Security Council;

8. Recalls the obligation of States to cooperate with the Tribunals, and in particular to comply without undue delay with requests for assistance in the location, arrest, detention, surrender and transfer of accused persons;

9. Decides that all States shall cooperate fully with the Mechanism in accordance with the present resolution and the Statute of the Mechanism and that consequently all States shall take any measures necessary under their domestic law to implement the provisions of the present resolution and the Statute of the 
Mechanism, including the obligation of States to comply with requests for assistance or orders issued by the Mechanism pursuant to its Statute;

10. Urges all States, especially States where fugitives are suspected to be at large, to further intensify cooperation with and render all necessary assistance to the Tribunals and the Mechanism, as appropriate, in particular to achieve the arrest and surrender of all remaining fugitives as soon as possible;

11. Urges the Tribunals and the Mechanism to actively undertake every effort to refer those cases which do not involve the most senior leaders suspected of being most responsible for crimes to competent national jurisdictions in accordance with their respective Statutes and Rules of Procedure and Evidence;

12. Calls upon all States to cooperate to the maximum extent possible in order to receive referred cases from the Tribunals and the Mechanism;

13. Requests the Secretary-General to implement the present resolution and to make practical arrangements for the effective functioning of the Mechanism from the first commencement date referred to in paragraph 1, in particular to initiate no later than 30 June 2011 the procedures for the selection of the roster of judges of the Mechanism, as provided in its Statute;

14. Requests the Secretary-General to prepare, in consultation with the Security Council, an information security and access regime for the archives of the Tribunals and the Mechanism prior to the first commencement date referred to in paragraph 1;

15. Requests the Tribunals and the Mechanism to cooperate with the countries of the former Yugoslavia and with Rwanda, as well as with interested entities to facilitate the establishment of information and documentation centres by providing access to copies of public records of the archives of the Tribunals and the Mechanism, including through their websites;

16. Requests the President of the Mechanism to submit an annual report to the Security Council and to the General Assembly, and the President and the Prosecutor of the Mechanism to submit six-monthly reports to the Security Council on the progress of the work of the Mechanism;

17. Decides that the Mechanism shall operate for an initial period of four years from the first commencement date referred to in paragraph 1, and to review the progress of the work of the Mechanism, including in completing its functions, before the end of this initial period and every two years thereafter, and further decides that the Mechanism shall continue to operate for subsequent periods of two years following each such review, unless the Security Council decides otherwise;

18. Underlines its intention to decide on the modalities for the exercise of any remaining residual functions of the Mechanism upon the completion of its operation;

19. Decides to remain seized of the matter. 



\section{Österreich im Sicherheitsrat der Vereinten Nationen}

- Österreichs Beitrag zur Aufrechterhaltung des internationalen Friedens und der Sicherheit

- Österreichischer Vorsitz in den Sanktionenkomitees zu Al-Qaida/Taliban und zum Sudan, sowie in der Arbeitsgruppe Internationale Tribunale

- Unterstützung des Sudan vor dem Unabhängigkeitsreferendum

- Einsatz für dauerhaften Frieden im Nahen Osten, Iran und Irak

- Entwicklungen in Krisenregionen: Somalia, Demokratische Republik Kongo, Tschad, Afghanistan, Demokratische Volksrepublik Korea, Sri Lanka, Haiti

- Friedensoperationen der Vereinten Nationen

- Österreich als Plattform für Dialog und Frieden

- Österreichs Vorsitz im Sicherheitsrat

- Schutz der Zivilbevölkerung in bewaffneten Konflikten, Menschenrechte

- Frauen, Frieden und Sicherheit, Bekämpfung sexueller Gewalt

- Kinder und bewaffnete Konflikte, Rechte der Kinder

- Stärkung der Rechtsstaatlichkeit, Bekämpfung der Straflosigkeit, Fortführung der Arbeit der Straftribunale

- Maßnahmen im Kampf gegen den Terrorismus und das organisierte Verbrechen

- Globale Abrüstungsbestrebungen

- Stärkung der EU-Außenpolitik und EU-interne Zusammenarbeit im Sicherheitsrat

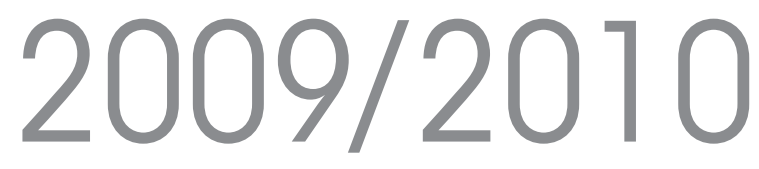

Bericht des Bundesministers für europäische und internationale Angelegenheiten 